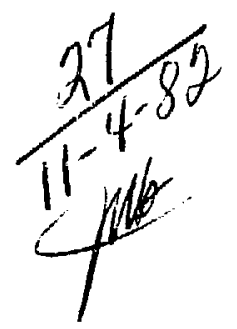

16262

\title{
Dr. 961
}

\section{Development of a \\ Magnetohydrodynamic Code for Axisymmetric, High- $\beta$ Plasmas with Complex Magnetic Fields}

Grant O. Cook, Jr.

(Ph.D. Thesis)

December 1982 


\section{DISCI.AIMER}

This document was prepared as an account of work sponsored by an agency oi the l nited Sitates fiovernment. Neither the United States Government nor the Liniversity of California nor any of their employees, makes any warranty, express or implied, or assumes any legal liability or responsibility for the accuracy, completeness, or usefulness of any information, apparatus, product, or process disclosed, or represents that its use would not infringe privately owned rights. Reference berein to any specific commercial products, process, or service by trade name, trademark, manufacturer, or otherwise, does not necessarily constitute or imply its endorsement, recommendation, or favoring by the Linited States Government or the I niversity of ( alifornia. The views and opinions of autbors expressed herein do not necessarily state or reflect tbose of the I nited States Government thereof, and shall not be used for advertising or product endorsement purposes. 


\title{
Development of a Magnetohydrodynamic Code for Axisymmetric, High- $\beta$ Plasmas with Complex Magnetic Fields
}

\author{
Grant O. Cook, Jr. \\ (Ph.D. Thesis)
}

Manuscript date: December 1982

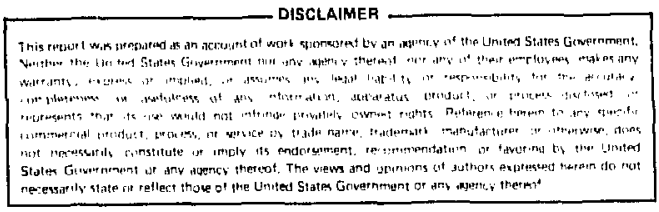

\section{LAWRENCE LIVERMORE LABORATORY University of California $\bullet$ Livermore, California $\bullet 94550$}




\title{
DEVELOPMENT OF A MAGNETOHYDRODYNAMIC CODE
}

\author{
FOR AXISYMMETRIC, HIGH- $\beta$ PLASMAS
}

WITH COMPLEX MAGNETIC FIELDS

A Dissertation

Presented to the

Department of Physics and Astronomy

Brigham Young University

In Partial Fulfillment

of the Requirements for the Degree

Doctor of Philosophy

by

Grant 0. Cook, in.

December 1982 
'This Dissertation, by Grant 0 . Cook, Jr., is accepted in its present form by the Department of Physics and Astronomy of Brigham Young University as satisfying the dissertation requirement for the degree of Doctor of Philosophy.

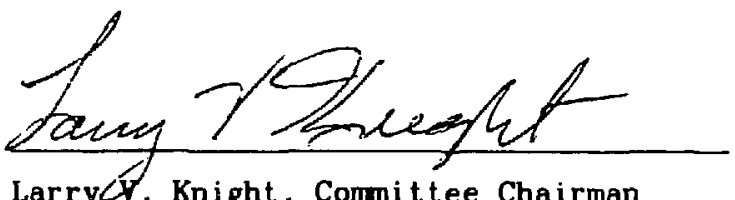

Larry C $\mathcal{H}$. Knight, Committee Chairman

Stow nd B Vanphet

Howard B. Van leet, Commit tee Member

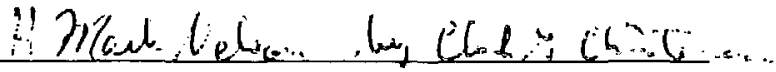

H. Mark Nelson, Commit tee Member

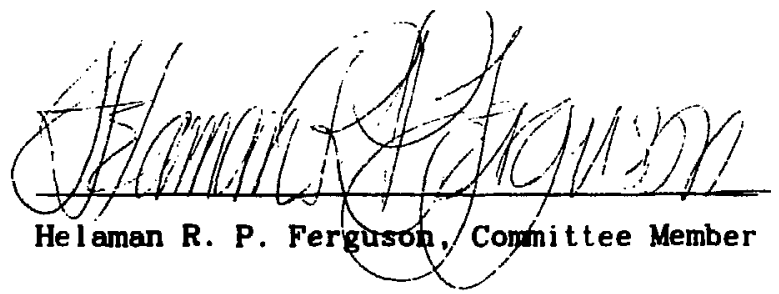

Sept 91982

Show ne \& Conpthed

Date

Howard B. Vanfleet, Department Chairman

$-\mathrm{i} i-$ 


\section{CONTENTS}

\section{Page}

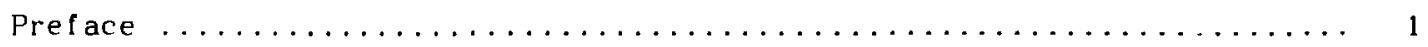

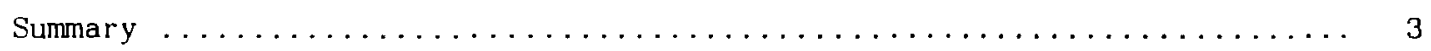

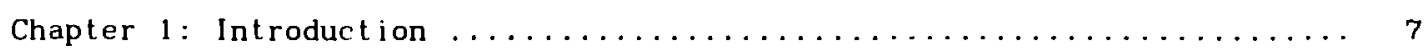

1.1 Overview $\ldots \ldots \ldots \ldots \ldots \ldots \ldots \ldots \ldots \ldots \ldots \ldots \ldots \ldots \ldots \ldots \ldots \ldots \ldots$

1.2 Physical Model $\ldots \ldots \ldots \ldots \ldots \ldots \ldots \ldots \ldots \ldots \ldots \ldots \ldots \ldots \ldots$

1.3 Finite-Dimensionalization and Solution Methods $\ldots \ldots \ldots \ldots \ldots$

1.3.1 Spatiai and Temporal Discretization $\ldots \ldots \ldots \ldots \ldots \ldots \ldots$

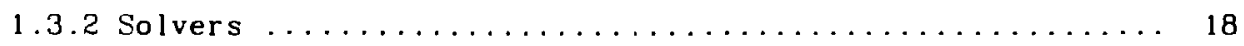

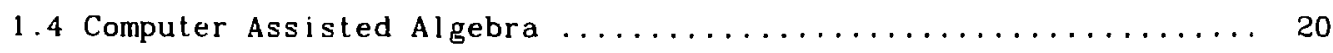

1.5 Operating Environment and Post-Processing $\ldots \ldots \ldots \ldots \ldots \ldots \ldots \ldots$

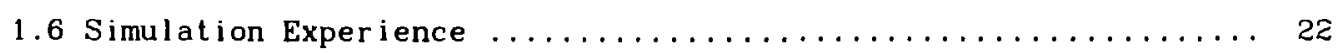

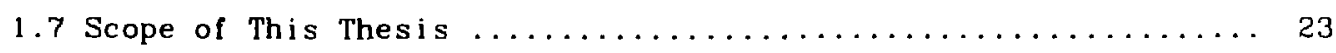

Chapter 2: Description of the Model $\ldots \ldots \ldots \ldots \ldots \ldots \ldots \ldots \ldots \ldots \ldots \ldots$

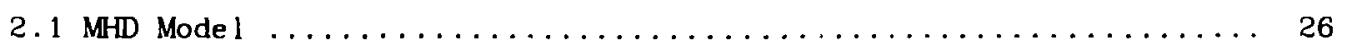

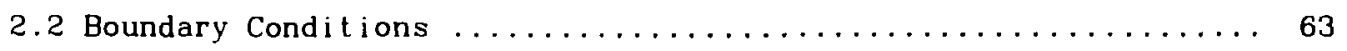

$2.3 \ln i t$ ial Conditions $\ldots \ldots \ldots \ldots \ldots \ldots \ldots \ldots \ldots \ldots \ldots \ldots \ldots \ldots$ 


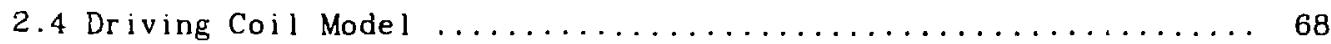

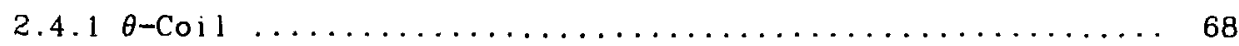

2.4 .2 Toroidal Coils $\ldots \ldots \ldots \ldots \ldots \ldots \ldots \ldots \ldots \ldots \ldots \ldots \ldots \ldots$

Chapter 3: Spatial Discretization Methods $\ldots \ldots \ldots \ldots \ldots \ldots \ldots \ldots \ldots \ldots$

3.1 General Spline Methods $\ldots \ldots \ldots \ldots \ldots \ldots \ldots \ldots \ldots \ldots \ldots \ldots \ldots$

3.2 A Two-Dimensional Spline $\ldots \ldots \ldots \ldots \ldots \ldots \ldots \ldots \ldots \ldots \ldots \ldots$

3.3 Discretization of the MHD Equations $\ldots \ldots \ldots \ldots \ldots \ldots \ldots \ldots \ldots$

3.4 Spatial Discretization of the Coil Equations $\ldots \ldots \ldots \ldots \ldots \ldots 101$

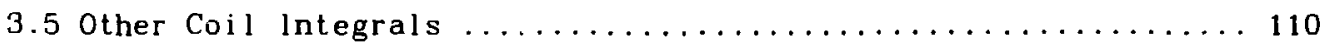

Chapter 4: Temporal Discretization and Solution Methods $\ldots \ldots \ldots \ldots \ldots 112$

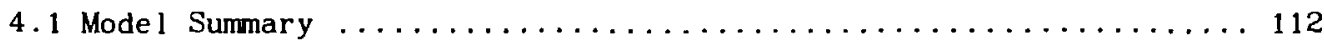

4.2 lmplicit Temporal lntegrators $\ldots \ldots \ldots \ldots \ldots \ldots \ldots \ldots \ldots \ldots \ldots$

4.3 Explicit Time Integration Techniques $\ldots \ldots \ldots \ldots \ldots \ldots \ldots \ldots$

4.4 Fully Discretized Equations $\ldots \ldots \ldots \ldots \ldots \ldots \ldots \ldots \ldots \ldots \ldots \ldots \ldots$

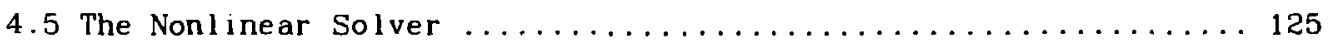

4.6 Tridiagoral Solver $\ldots \ldots \ldots \ldots \ldots \ldots \ldots \ldots \ldots \ldots \ldots \ldots \ldots \ldots \ldots \ldots \ldots$

Chapter 5: Automatic Coding of the Physical Model $\ldots \ldots \ldots \ldots \ldots \ldots \ldots \ldots$

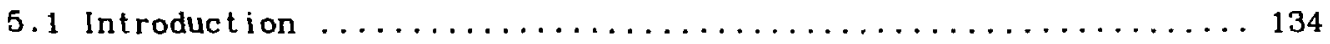

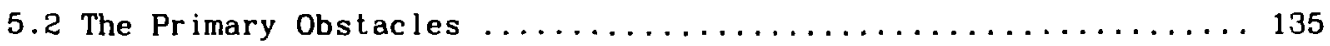

5.3 Reduction to Simplest Possible Terms $\ldots \ldots \ldots \ldots \ldots \ldots \ldots \ldots$

5.4 Efficient Internal Representations $\ldots \ldots \ldots \ldots \ldots \ldots \ldots \ldots \ldots \ldots 142$

5.5 Structuring of Results at Every Step $\ldots \ldots \ldots \ldots \ldots \ldots \ldots \ldots$

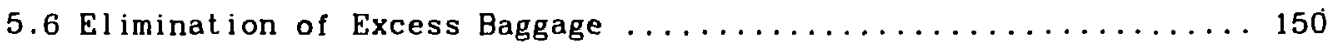


5.7 Fast Factoring and Optimization Functions $\ldots \ldots \ldots \ldots \ldots \ldots 2$

5.8 Use of Large Virtual Memory Computers .............. 162

Chapter 6: How PDEs are Transformed into FORTRAN $\ldots \ldots \ldots \ldots \ldots \ldots \ldots$

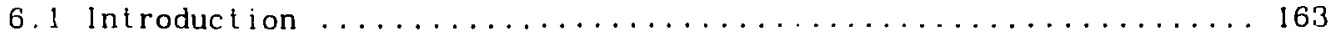

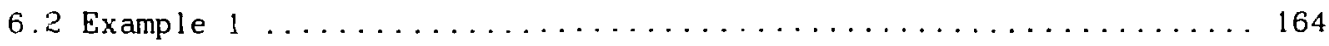

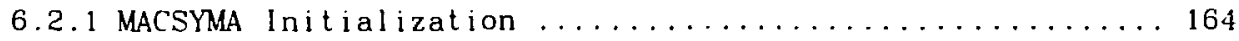

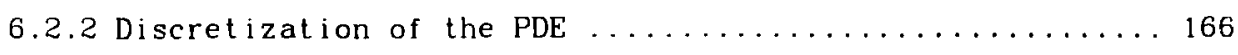

6.2.3 Jacobian Calculation and Code Generation ........... 173

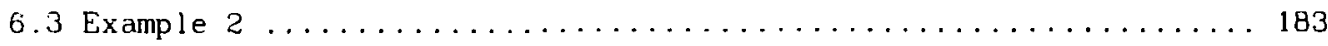

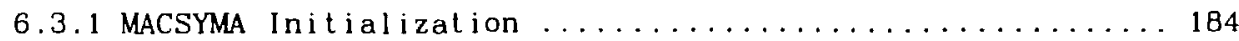

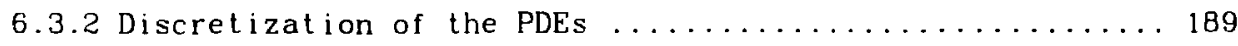

6.3.3 Jacobian Calculation and Code Generation .......... 197

6.4 The Complex MHD Model $\ldots \ldots \ldots \ldots \ldots \ldots \ldots \ldots \ldots \ldots \ldots \ldots \ldots$

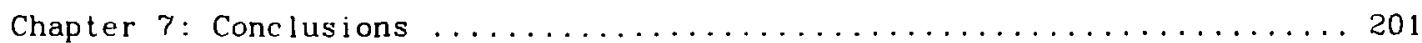

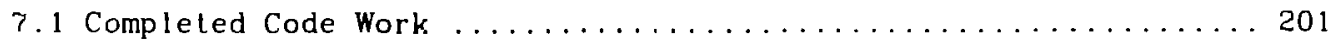

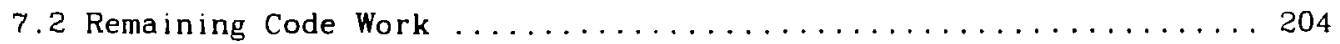

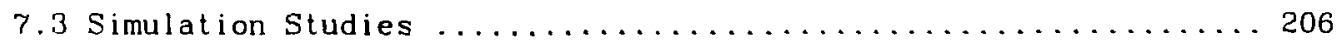

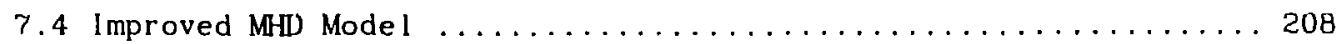

7.5 Better Numerical Techniques $\ldots \ldots \ldots \ldots \ldots \ldots \ldots \ldots \ldots \ldots \ldots$

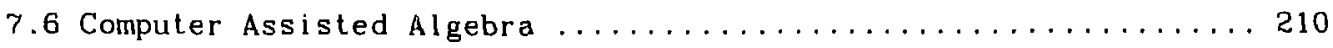

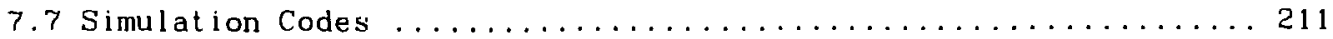

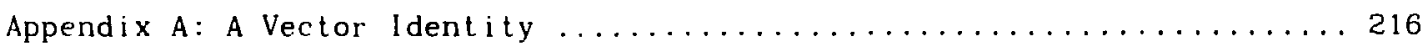

Appendix B: Spline Boundary Conditions $\ldots \ldots \ldots \ldots \ldots \ldots \ldots \ldots \ldots \ldots$ 
Appendix $\mathrm{C}: \mathrm{Bi}-\mathrm{Quint}$ ic Interpolant $\ldots \ldots \ldots \ldots \ldots \ldots \ldots \ldots \ldots \ldots \ldots \ldots \ldots$

Appendix D: Numerical Methods for the Complete Elliptic Integrals $K$ and $E$ 238

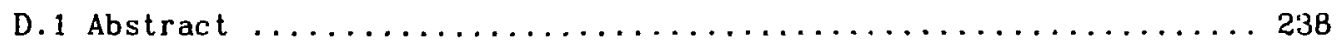

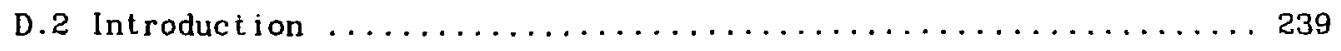

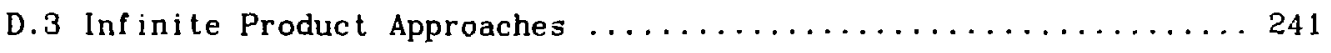

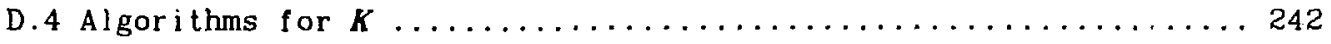

D.5 Comparison of the Algorithms for $k \ldots \ldots \ldots \ldots \ldots \ldots \ldots \ldots$

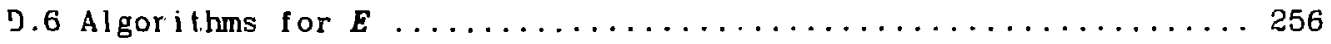

D.7 Comparison of the Algorithms for $E \ldots \ldots \ldots \ldots \ldots \ldots \ldots \ldots \ldots \ldots \ldots \ldots$

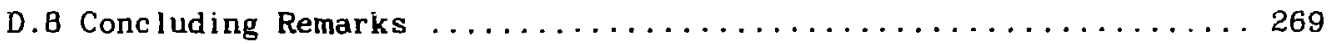

D.9 Acknowledgment $\ldots \ldots \ldots \ldots \ldots \ldots \ldots \ldots \ldots \ldots \ldots \ldots \ldots \ldots \ldots$

Appendix E: FORTRAN OUTPUT $\ldots \ldots \ldots \ldots \ldots \ldots \ldots \ldots \ldots \ldots \ldots \ldots \ldots \ldots$

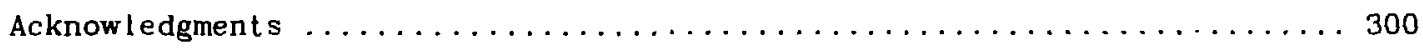

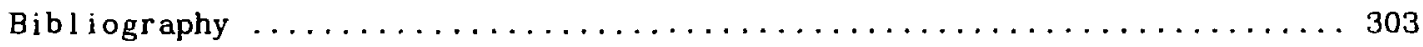




\section{PREFACE}

As an undergraduate student at Brigham Young University, 1 had the good fortune to become associated with the Topolotron project. This project studies a new magnetic fusion concept that is based on the idea of a two-dimensional structurally stable magnetic field. The challenging research 1 performed with others on the project stimulated my resolve to study plasma phenomena. I envisioned using a realistic physics computer code to model the developing Topolotron experiment.

The opportunity came in 1978 when Associated Western Universities extended a graduate fellowship to go to Lawrence Livermore National Laboratory. No computer code was sufficiently general to model the Topolotron, so I was given the challenge to modify the ANIMAL code, an implicit Eulerian two-dimensional magnetohydrodynamic computer code, to suit the.demands of the Topolotron.

Several months of elaborate hand algebra convinced me that it was not possible to perform the algebra necessary for the modification without introducing significant errors. Consequently. I decided to automate the algebra 
using MACSYMA, a computer algebra program, and thereby began developing an entirely new code. This thesis describes the development oi the rew code designed for the Topolotron. 


\section{SUMMARY}

Every code development project is motivated by a primary goal. l'mally. the goal is the elucidation of a physical property or class of phenmenn. Citical examination of a particular experiment is the objective in uthe? instances. Because of the substantial cost of realistic codes, especially multidimensional ones, very few have been developed for a specific experiment. I break with tradition here by creating computer algebra lools which automate the major tasks in a code development project, thereby making il feasible to design a code for a specific experir ... The experiment studied here $1 s^{\text {the }}$ Topolotron experiment.

The Topolotron is an axisymmetric, toroidal magnetic fusion concept in which two-dimensional effects are important, as well as all three magnetic field components. Lacking a code which could adequately handle this problem, the major components of a new MHD code have been developed using the original computer algebra tools mentioned above. The particular MHD model employed is basically the orie-fluid, two-temperature model using classical Braginsil Iransport with viscous effects ignored. The model is augmented by Saha-Boltzmain dissociation and partial ionization physics, a simple radiation 
loss mechanism, and an additional resistivity due to electron-neutral collisions. While retaining all velocity and magnetic ileld components, the assumption of axisymmetry is made, and the resulting equations are expanded in cylindrical cocrdinates. The major approximation technique is then applied: spline collocation, which reduces these equations to a set of ordinary differential equations.

Although the computational boundaries are assumed to be adiabatic walls, the reinaining boundary conditions are quite general. In particular, no assumption is made concerning, the magnetic field at the wall, and a mechanism for the plasma to bounce of the wall is incorporated into the boundary conditions. However, no detailed wali breakup dynamics are included.

The above models are coupled to a complicated external circuit calculation. One part of the external field is generated by a square toroidal coil: the poloidal current it carries generates a toroidal field. Two other coils carrying toroidal currents generate the poloidal field. While the square toroidal coil presents no particular difficulty, the other coils do. Traditionally, this problem would be solved by the method of moments. Instead, we develop a new technique based on B-splines which is far more accurate and efficient. It can be shown that this particular formulation leads to a pair of linear coupled first kind Fredholm integral equations with singular kernels. 
Solving the overall model is done in two steps. First, the coil calculation is advanced in time. Then, using the resulting external field as a driver, the plasma variables are found in the closed plasma domain. Each step is fully implicit, and because the MHD equations are highly nonlinear, a generalized Newton technique is employed to solve for the variables in the second step. In addition, as the MHD equations generate an enormous matrix system to be solved, an ADl-like matrix splitting is done to facilitate solving the equations in a reasonable length of $t$ ime.

Experience has proven that many techniques commonly used in the solution of partial differential equations are unstable or marginally stable at best. For this reason, a relatively new temporal integration technique is employed: a two-step one-leg method. A quintic spline with a compact matrix representation, the so-called "Spline 4" developed by Rubin and Khosla, is used to approximate the spatial derivatives. This representation also permits a numerically stable "upwinding" approximation to spatial first derivatives without any formal loss of accuracy.

In order to transform the above theoretical models into FORTRAN statements, an enormous amount of algebra had to be done, the bulk of it occurring in a Jacobian calculation for the MHD equations. Using MACSYMA and adding new powerful capability, a MACSYMA code was developed which performed all of the algebra for the MHD model. Another MACSYMA program generated the code fragments required in the coil calculaiion. The new MACSYMA system tools 
developed are of sufficient generality to apply to other sets of partial differential equations, anticipating the inevitable improvement in the MHD modelling of plasmas. 


\section{CHAPTER 1}

\section{INTRODUCTION}

\subsection{Overview}

Only in the past decade has multidimensional simulation of complex physical phenomena come of age, and with it the ability to perform computer calculations describing plasma behavior. However, this new field of computational physics, and especially computational plasma physics, has experienced considerable growing pains, primerily because many finite-dimensional numerical discretizations introduce numerical instabilities which can be difficult to distinguish from physical instabilities characteristic of the infinite-dimensional model. Further difficulties face the computational physicist as the lack of sufficient computer power forces upon him a set of physical and numerical approximations he may have preferred to avoid. And then there is the algebra required to transform whatever physical model he has settled upon into FORTRAN code, a task which grows exponentially in complexity with linear increase in the size of the modelling partial differential 
equations. The code that results must be augmented by an adequate operating system and post-processing capability. After the computer program has been "shaken down," work can finally begin! This work consists of making a series of runs to cover the cases of physical interest, and then in interpreting the results, a sequence very similar to the procedures followed by experimental physicists. Therefore, the computational physicist's domain overlaps that of the theoretician and the experimentalist, retaining however some unique tasks.

While dramatic increase in computer power and more modest improvement in programming support will surely proceed apace, they are as yet insufficient to meet the computational physicist's demands. So approximations and simplifications will necessarily continue at all levels of work leading to computational experimentation.

Within this framework, I reviewed the work done in time development studies of medium-density high- $\beta$ plasmas. This review covers five importani aspects. First, what physical model has been used? Second, what kinds of discretization and linearization have been employed? Third, has the algebra involved been automated, and if so, is it done efficiently? Fourth, what has been provided for the code's operating environment and data reduction or post-processing phase? And finally, what was learned through the computational experiments? In this chapter, answers to these questions will be presented. 
Although this thesis has wider applicability, particularly to magnelic fusion work, it was motivated by the magnetic fusion cuncept called the Topolotron. In general, a Topolotron is an axisymetric, toroidal magnetic fusion device with a poloidal plasma cross section that is non-circular. Its name is derived from the fact that in the sharp boundary limit, the $B$-field on the surface or boundary of the plasma is structurally stable; i.e.. the two-dimensional $B-f$ ield does not change topological type. The reason for this is that the field lines are neither ergodic nor closed (as is the case in many magnetic (usion devices), but asymptotic to a limit cycle. In other words, the B-field lines approach a single closed field line in such a way that perturbations of the overall field structure do not change the topological type of the field. If the plasma boundary becomes diffuse, it is no longer clear to what degree stability is maintained. There remains the question of precisely how a structurally stable $B$-field is estabiished in time from an infinite set of possible initial conditions. In order to study these stability questions. computer simulations must be performed. This study develops the tools to initiate further studies through simulations. 


\subsection{Physical Model}

The first important aspect in computationally studying high- $\beta$ plasmas is what mathematical model best represents the physics occurring in these plasmas. Models range from a simple snowplow model of the plasma compression ${ }^{85}$ to one involving a number of detailed transport mechanisms. ${ }^{90}$ Ideal MHD codes, the most frequently occurring in the literature, constitute an intermediate approach. Of the many which could be cited, Lui and Chu's ${ }^{77}$ single-fluid ideal MHD code was used for screw pinch studies. The great variety in the models used in the literature is motivated most often by a desire to account for some additional physical effect. Occasionally, however, new codes have arisen from a need to improve the numerical methods used.

For example, experience has shown that explicit treatment of time derivatives excessively limits the time slep that can be taken in integrating initial-value problems, so Lindemuth ${ }^{60}$ developed an implicit code, the first for noulinear multidimensional MHD problems. ANIMAL ${ }^{71}$ was a specialization of that code to one magnetic field component and two velocity components with the inclusion of additional physical effects. While not as complete a model, Schnack's MHD-2D ${ }^{106}$ code was designed to study the nonlinear development of resistive tearing modes in MHD plasmas. Another code which was limiled to simple transport was Finan's IMP code. ${ }^{38}$ However, it was a very ambitious project because it is fully three-dimensional. 
Other codes incorporated special treatments of transport and radiation physics, while using a simple MHD model. For example. Davidson et al. ${ }^{27}$ incorporated microturbulence enhanced transport into the MHD equations. More precise modeling of ionization and radiation for optically thin plasmas was added to the ifeal MHD equations in the code of Duston and Duderstadt. 33 Another class of of codes is built on the special ordering of terms which can be done for low $\beta$ conditions. See Killeen's excellent reviews for more background on these and other codes of the last two decades. ${ }^{60.61}$

Many codes are now available with a wide range of capabilities. However, there is only one nonlinear multidimensional MHD code with substantial transport physics built into it, and that is LASNEX. ${ }^{90}$ Unfortunately the full treatment of all three magnetic field components was only recently added, which was the underlying motivation for the development of a new code. This thesis presents several extensions and novel features which LASNEX does not possess. However, the effort here was not intended for laser-plasma interaction studies, so, for example, radiation transport is not an issue with which we will deal.

Another important facet of plasma simulation codes is the selection of boundary conditions. To the best of my knowledge, all of these codes use simple boundary conditions, in some cases, non-physical ones such as assuming an insulating boundary is simultaneously a perfect conductor. ${ }^{72}$ On the other hand, a novel physical boundary condition was implemented into ANIMAL; it allowed for plasma-wall separation as well as plasma bounce effects. 
In magnetic fusion experiments, the driving magnetic field arises from an external source which must also be modelled. Two examples are described here. First, continuous metal conductors were modelled by Nelson, Brown, and Hart $\mathrm{t}^{85}$ as a finite number of filmentary wires. In the second case, the MFTF-B, the steady state of the superconducting coils is of primary interest, so the external field need be computed only once for a given experinent. Although the conductors have much larger cross-sections in this instance, the steady-state conditions make the assumption of uniformly distributed current in each conductor a good first approximation. 103

\subsection{Finite-Dimensionalization and Solution Methods}

Computer simulation of the physical models discussed in the last section involves discretization of partial differential equations and sometimes integro-differential equations. If the resulting discrete algebraic equations are nonlinear, some sort of linearization process must be done to accommodate a Newton-type nonlinear equation solver, or some other nonlinear equation solver. Volumes have been written on the various discretization and solution techniques which have been developed, and new methods are constantly appearing in the literature. Consequently, the descriptions given here reflect only a partial overview of these fields. 


\subsubsection{Spatial and Temporal Discretization}

Basically two points of view deal with the spatial discretization problem, the Eulerian and Lagrangian techniques. In the former, the computational grid is generally fixed, while in the latter, this grid moves with the fluid. Both suffer drawbacks. For the Eulerian grid, it is the inability of the mesh to resolve regions of steep gradient. In the Lagrangian grid, the mesh is highly distorted and tangled in cases of highly compressible flow. Recent developments in both approaches are attempting to overcome these primary difficulties. For the Eulerian grid, the developments consist of a number of moving grid and grid refinement ideas, the most impressive of which is Brackbill's idea to solve a variational problem to relocate a non-orihogonal mesh at every time step (or at least frequently). ${ }^{116}$ Winslow, who calls this type of approach "adaptive mesh motion" (AMM), has proposed a sinilar technique which should also work in untangling distorted meshes in Lagrangian methods. Another class of methods has been characterized as "adaptive mesh refinement" (AMR) techniques. ${ }^{10}$ While possessing the advantage of being able to vary the number of mesh points to exactly suit the problem at hand, 45 Kansa has pointed out that there are complex questions about the vectorization potential of such methods. ${ }^{5}$ Hedstrom and Rodrigue argue that the computation on each fine grid could proceed simultaneously on different components of a multiprocessor. This process is definitely feasible for explicit time-integration methods, but the detailswill be extremely complex, especially as more and more detailed 
transport is included in the physical model. A* this area of research is just beginning to mature, and multiprocessors are just becoming available, the relative merits of the best AMM and AMR approaches and possibly a hybrid technique will not be fully assessed for some years to come.

Once the underlying finite-dimensional grid has been choser, a decision must be made regarding the means of approximating the partiai differential equations on that grid. There are three general classes of spatial approximation: first and most popular, the flux-form finite difference method: ${ }^{70}$ second, the point collocation method; and finally, the full Galerkin finite element technique. Because of the expense involved with full finite element techniques, they have seen use in only the simplest fluid dynamics problems. The techniques in the second class, the collocation methods, are in reality a special case of the finite element method where the weighting functions or trial functions are linear combinations of delta functions. ${ }^{3}$ They have become more popular just recently with the development of the so-called "compact," higher-order spatial operator discretization methods. ${ }^{35}$ The term "compact" conveys the idea that these special piecewise polynomials lead to very narrow bandwidth Jacobians in a Newton solver of the discrete model. This is in contrast to the idea of "compact support" in the theory of B-splines." "Conpact" discretization methods have seen only limited use because of the misconception that they involve more computational expense. As for "flux-form" or "conservation form" finite differencing, much has been written, especially in 
the classic texts on computational physics. ${ }^{95.96}$ The idea is to transform all equations so that all derivative terms can be viewed as fluxes of physical quantities. Integrating over each subdomain then leaves only the computation of interface-type quantities. There are terms for which this is not possible because they do not represent fluxes. Therefore, in most complex models, there will be approximation inconsistencies when using the "conservation form" method.

Because boundary conditions also involve the approximation of spatial operators, they come under the same general approximation category as the interior partial differential equations (PDEs). In fact, if their treatment is inconsistent with the approximation method on the interior spatial domain, serious numerical errors will propagate into the interior region. ${ }^{9.114}$ By their intrinsic nature, however, boundary conditions must be treated in a different but consistent manner. A host of extrapolation and one-sided differencing methods fill the literature for boundary derivatives. ${ }^{6}$ Other methods involve "ghost points"38 or "artificial points", that is, approximations which require points outside the computational domain. ${ }^{109}$

The final differential operators are the time derivatives. Approximation techniques here are distinguished by whether they are implicit or explicit, that is, by whether the rest of the terms in the equation are evaluated at the advanced time level (the level being evaluated) or at some past time level (explicit). The picture is complicated by the existence of techniques which evaluate some terms at past time levels and others at the 
advanced time levels. This "time-splitting" or introduction of intermediate time levels is motivated either by the intrinsic complexity of the equations making their coupled solution far too expensive, or by the multidimensional nature of the problem. In the latter case, the derivative operators are separated by the "direction" in which they operate (mixed derivatives are treated explicitly) and a "time-level" assigned to each. The resultirg alternating-direction implicit algorithm (ADI) involves solving each one-dinensional problem using information from the previous steps in a cyclic manner. The algorithm suffers from an inability to model time-dependent boundary conditions at the intermediate time levels to better than first-order accuracy. ${ }^{11}$

For methods which don't involve these additional multi-iniermediate time-level complexities, the spatially discretized partial differential equations can be viewed as a set of ordinary differential equations (ODEs). Then, the very extensive theory covering ODEs can be invoked. Both explicit and implicit techniques can involve two or more time levels, but because of excessive storage requirements, large scale physics codes generally do not use more than three time-level temporal integrators. When the time scales in the physics problem don't vary over too many orders of magnitude, explicit techniques have proved to be a viable approach. However, if there is a wide range of time scales, then the problem is termed "stiff" and it is essential to use a stable implicit integrator. Included among the many classes of temporal 
integrators are linear multistep methods (LMMs), one-leg methods (OLMs), and Runge-Kutta methods. There are explicit and implicit techniques in each category with most of the commonly used temporal integrators being LMMs. As far as "stiff" (A-stable) integrators are concerned, there are such methods in each above named category. A method is A-stable with respect to a given test equation if the solutions obtained by using the method to integrate the test. equation are always bounded. whenever the eigenvalue of the lest equation has negative real part. The standard test equation is (4.10). Examples of some A-stable methods in the LMM class are the backward differentiation methods (BDFs). Another A-stable LMM is the trapezoidal rule, sometimes known as the Crank-Nicolson method. It is the most widely used technique in the large scale MHD codes discussed earlier. Variants of the trapezoidal rule which weight the past time level more heavily make up another large segment of methods. Beam and Warming have done an excellent job of pointing out the strengths and weaknesses of LMMs and the newly discovered OLMs ${ }^{4}$ in the context of fluid dynamics. "Predictor-corrector" variants of implicit methods have also been popular. 109 Spatial discretizing the coil equations has effectively been a zero-order approximation; that is, the current in the conductor is assumed to be constant over each region between the mesh points defined by the discretization of this problem. This technique is termed the "method of moments"82 and has also been used in many other applications, primarily in mechanical engineering. 
The assumption of uniformly distributed currents is equivalent to using step functions as basis functions. ${ }^{103}$ More complex two- and three-dimensional basis functions have been used in magnet design. ${ }^{80}$ While this approach has to be carefully monitored in initial-value MHD problems due to the need to repeat the calculation many times, it can be more efficient to use higher-order basis functions. Each of these techniques is a special case of the weighted Galerkin f inite element method.

\subsubsection{Solvers}

For the cases of explicit time integrators, including the "predictor-corrector" variety, no linear system solution is required. On the other hand, implicit integrators always lead to a system of linear or nonlinar algebraic equations to solve. As nonlinear problems can be regarded as a sequence of linear problems depending upon the strategy used to solve them, we discuss linear systems solvers first. The Gauss-Seidel method can solve linear systems. However, this is usually far too costly. So, for exemple, advantage is taken of the sparsity of the matrix in scalar and block tridiagonal solvers, the kind most of ten called for by the spatial discretizations used in the MHD equations. In multidimensional problems, this sparsity pattern is not preserved, and ADI and matrix splitting approximations are applied to obtain a series of effectively one-dimensional linear systems which have the original sparsity pattern. ${ }^{4}$ For 9-point finite differencing of mixed derivatives, no such convenient approximations are available, and so another type of linear systems 
solver has been pursued: incomplete Cholesky conjugate gradient (ICCG) algorithms. There are difficulties with these methods when the matrix involved is non-symmetric, and there has been a substantial research effort going on to overcome this obstacle.

For nonlinear problems, there are a number of different strategies of varying practicality. One of the simplest is the secant method. ${ }^{4}$ The Newton method comes next in complexity, requiring a Jacobian update at each iteration. A great numbs of quasi-newton and variable-metric strategies come in between these two, the main idea being to avoid recomputing the Jacobian at each step if possible.

While the above methods are generally adequate, they do not always converge: they are only guaranteed to converge if the starting point is "sufficiently close" to the solution vector. One means around this has been the variable step-length Newton methods of Hirsch and Smale ${ }^{4}$ as well as those of Wacker, Zarzer and Zulehner. ${ }^{111}$ Again, these are not guaranteed to converge, but appear to possess excellent practical properties. Another set of techniques which is guaranteed to find the solution with "probability one" has been developed only recently.115 These are the "homotopy continuation" methods. While possessing great theoretical adyantages, these methods are expensive computationally and have not been used in large-scale physics codes. 
In spite of the occasional convergence difficulties experienced by major MHD codes, 72 it appears that the improved "generalized Newton" algorithms of Hirsch and Smale and others have not come into use in large-scale physics codes. If a sincere altempl is being made to follow the phase space trajectory of an initial value problem, then it would seem that more computational expense should be incurred to obtain convergence at each step through phase space.

\subsection{Computer Assisted Algebra}

As those who have worked with complex MHD models are well aware, there is an enormous amount of algebra to be done in performing the discretization and linearization of the model equations, not to mention any grouping, factoring, or optimization of the final expressions. To answer this obvious need, some work has been done to assist the physicist over this arduous, mundane, and error-prone task. The recent thesis of Wirth ${ }^{117}$ represents perhaps the best of these efforts. In addition to systems described in the references cited by Wirth is the ARIEL interactive system of Mikhailov and Aladjem. ${ }^{83}$ Also, Finan ${ }^{38}$ automated the computation of the Jacobian elements for his three-dimensional MHD model, a process which presumed that the component form of the model equations had been computed correctly. There are indubilably numerous other systems for performing the various algebraic tasks in Jeveloping a large physics code. However, except for Wirth's tools, they have all been rather narrow in their approach and capability. And curiously, in spite of the substantial advance 
which Wirth's work represents, it was still only possible to altack small sets of partial differential equations, certainly nothing of the scale currently being implemented into MHD codes.

\subsection{Operating Environment and Post-Processing}

The operating environment is another neglected area of research, at least in the open literature. Wirth's thesis, for example, delves into this matter at some length; others have dealt with it briefly. ${ }^{34.78}$ Nonetheless, the operating environment is of practical importance because the development of subroutines to perform these tasks is a substantial effort. The particular areas comprising the operating environment are run initialization logic, monitoring facilities including debugging capability, database mainienance. shutdown and restart capability, and finally time-step control. ${ }^{38}$ Time-step control overlaps into physical and numerical considerations as well. As for the operating environment aspects of time-step control, users sometimes wish to restrict the time step based on arbitrary criteria they wish to communicate to the code either initially or as the simulation develops. These unforsee.s conditions make it proper to include time-step controller logic in the category of operating environment.

Large scale physics codes generate an unwieldy amount of data which needs to be reduced to a form that will give guidance and insight lo the physicist. In other words, the purpose of all this computing is insight, not 
thousands of pages of numbers $!^{44}$ Accordingly, the post-processor must have the facility to present portions of this huge database in a number of graphical forms and the capability to integrate and perform other special manipulations on the data so as to permit comparison against experimental data. ANIMAL ${ }^{72}$ has a post-processor which, given the MHD model used, is geared to provide just such information. It also has sophisticated graphic analysis capability. While other MHD codes provide similar functionality, 90 this processing power has only been developed at great cost and with limited generality. The same is true for the code comprising the operating environment.

\subsection{Simulation Experience}

Computational MHD results are now manifold. Even in multidimensional problems, several published simulation studies using ANIMAL, ${ }^{2,74.75}$ LASNEX, ${ }^{90}$ $I M P,{ }^{38}$ and others ${ }^{107}$ have already appeared. Nonetheless, this is still virgin territory because these simulations are very expensive. And while we can count on the continued improvement in capability and reduction in expense of large computers, means of better focusing these resources is always needed. Improvements of the efficiency and accuracy of the above-mentioned codes as well as those under development is one means of achieving a better focus.

Insofar as the Topolotron is concerned, only a simple MHD snowplow simulation has been done. ${ }^{86}$ While this initial computational step appeared to verify that an equilibrium Topolotron configuration can be attained from an 
initially uniform neutral gas. the physical detail is much too skelchy. In contrast. Schnack ${ }^{106}$ studied details of the tearing mode in the Reverse Field Theta Pinch: Nielsen ${ }^{90}$ investigated the radiation-enhanced collapse of a Z-pinch: and Lindemuth et al ${ }^{74}$ discovered that plasma-wall interactions can initiate unstable plasma oscillations. There are now many additional examples which could be given, and they all indicate that inclusion of more physical effects in the MHD models leads to an improved understanding of the qualitative behavior of medium-density plasmas.

\subsection{Scope of This Thesis}

As discussed, there is ample work in computational plasma physics to keep physicists working for some years to come. Solutions to every difficulty delineated in the above discussion will not be provided in this thesis. However, several areas will receive considerable attention.

In Chapter 2, an adequate physical model for the Topolotron and other similar experiments is derived. The final equations are believed to be the most ambitious tackled computationally to date.

Chapters 3 and 4 deal with the numerical approximations required to solve these equations. Chapter 3 emphasizes the spatial discretization of both the MHD and coil equations. New developments here are the introduction of a "natural" upwinding scheme which has the same order of accuracy as the spline first derivative approximation and is therefore consistent with the order of 
approximation of the second derivatives and dependent variables. I discovered consistent numerical approximations for the spline derivatives at the boundaries. This is essential if the approximation to the physical boundary conditions is to be consistent with the approximation of the interior equations. Next, I found a new way of performing the coil calculation, including highly accurate evaluation of the singular integrals which arise. Finally, a description of how the magnetic flux integrals are performed and how the external field and its derivatives are computed is given. Chapter 4 details how the sparially discretized system, a set of ODEs with constraints, is time discretized to permit its formal time integration. Chapter 4 explains the means of solving the equations which result when the algebraic model is complete.

Chapter 5 gives an overview of how the required algebra was performed using MACSYMA. The viewpoint taken is that one should be able to start with integro-differential equations and end up with optimal FORTRAN code segments. The key in achieving this goal was to tackle only the specific algebraic problems at hand, and not to attempt to provide tools to cover every conceivable numerical scheme. Also of great importance was the optimization that was done on the performance of several MACSYMA functions I added.

Chapter 6 gives two detailed examples of this transformation from differential equations to FORTRAN code. The first is a single, trivial partial differential equation which nonetheless illustrates the power of this approach. By way of contrast, the second example is an ideal MHD model similar to that of 
Lui and Chu. ${ }^{77}$ While only a portion of the required algebra could be performed for this model on the "limited-memory" DEC-10 computer at the Massachusetts Institute of Technology (MIT). this example gives a little better flavor for the magnitude of the algebra involved in turning the model of Chapter 2 into FORTRAN code segments. Also included is a discussion of what additional facilities are required to do the astronomical amount of algebra our model implies.

Finally, Chapter 7 discusses the weaknesses in current MHD theory. numerical approximation theory, and automatic code generation capabllity. Suggestions are given for means of overcoming these weaknesses with particular emphasis on the implications for computer-assisted code generation. The construction of the code to perform simulations of the Tupolotron was not completed. Comments on the completion of the code and what could be learned from it about the Topolotron conclude this work. 


\section{CHAPTER 2}

\section{DESCRIPTION OF THE MODEL}

\subsection{MHD Model}

In order to perform a numerical simulation of a plasma experiment, an appropriate model must be developed. This is a difficult task because the class of phenomena occurring in plasmas is so complex. While not taking account of impurity radiation losses, microturbulence-driven anomalous transport, and heat loss to the wall, the model derived here will be an improvement over those previously considered which are appropriate for a lluid-regime approximation. ${ }^{38,72,73,106}$

To guide this approximation process, the parameters of the current Topolotron experiment are needed. Other pinch experiments, future Topolotron experiments in particular, should also be considered. In the sequel, hever. the cail names will be those of the present experiment. There are three such coils, and they all have approximately toroidal symetry. First, the $\theta$-coil is 
the outer coil and it carries a poloidal current. When viewed in the poloidal cross section of the device. it has a rectangular shape which surrounds the other coils and the vacuum vessel. The other two coils carry toroidal currents (opposite from one another). The larger of the two, the C-coil, is the farthest from the main axis of symmetry and is shaped like a backward $C$ with sharp corners. Vertically oriented, the smaller $I$-coil is simply a straight conductor when viewed in the poloidal plane.

With this description in mind, consider some experimental parameters. On the current Topolotron experiment, the $B$-field ranges up to $5 \mathrm{kG}$, the particle density, $n$, ranges up to $10^{16} / c c$, the $\theta$-coil current peaks at $800 \mathrm{kA}$ with a voltage of $30 \mathrm{kV}$, the $C$-coil current peaks at $100 \mathrm{kA}$ with a voltage of 30 $\mathrm{kV}$, and the $I$-coil current peaks at $70 \mathrm{kA}$ with a voltage of $40 \mathrm{kV}$. The electron and ion temperatures are not known, but presumably are in the range of $10^{-2}$ to $10^{-1} \mathrm{keV}$. with the electron temperature being usually much larger than the ion temperature. In future Topolotron experiments, it is anticipated that these parameters, particularly the $B$-field and the temperatures, would be scaled up towards those required for fusion conditions. It is not presently known what modifications will be made to the conductors bearing toroidal currents. Presumably, studies based on the work done here would guide such decisions.

From these estimates for the present experiment, we can deduce several plasma parameters which are going to play an important role in the subsequent analysis. These parameters are the electron cyclotron and plasma frequencies, 
the plasma skin depth and the electron mean free path. For the electron cyclotron frequency, we have

$$
\omega_{c e}=\frac{e B}{m_{e} c} \sim 10^{10} \mathrm{rad} / \mathrm{sec} \text {. }
$$

The electron plasma frequency will scale as

$$
\omega_{p e}=\sqrt{\frac{4 \pi n_{e} e^{2}}{m_{e}}} \sim 10^{12} \text { to } 10^{13} \mathrm{rad} / \mathrm{sec}
$$

A related quantity, the skin depth, is roughly

$$
\delta \equiv \frac{c}{\omega_{p e}} \sim 10^{-2} \text { to } 10^{-1} \mathrm{~cm}
$$

Finally, the electron mean free path is in the range $10^{-1}$ to $1 \mathrm{~cm}$.

While the scale lengths of interest in larger Topolotron experiments may not be as fine, it is presently desired to be able to resolve phenomena whose wavelength, $L$, is .5 centimeters or greater. As will be seen later, this can render suspect the neglect of some viscous forces, because the electron mean free path (and also the ion mean free path) are approaching the same order of magnitude as $L$. However, any errors introduced by this ordering are ignored here. 
Having characterized somewhat the class of experiments to be simulated. the actual model can be chosen and developed. There are several approaches to modelling the time development of a plasma. The principal variation involves whether the plasma is treated as a very large collection of particles or whether it is treated as a fluid. One class of techniques involves a hybridizing of the two approaches. For example, the electrons might be treated as a fluid and the ions and neutrals as collections of particles. Further variation in these techniques generally involves averaging the plasma properties, as in a new particle approach for plasmas with fairly uniform properties, ${ }^{23}$ or statistically sampling a physical process as is done in studying neutral beam interaction with a plasma via Monte Carlo techniques. ${ }^{18}$

Because of the high density of the main discharge in the Topolotron, I used a fluid approximation to the plasma behavior. Some have argued that while this is appropriate for the electron fluid, it is incapable of describing how heavy particles interact with shock waves. ${ }^{27}$ The evidence is not yet compelling enough to go to the more complex (and very expensive) hybrid code. It is possible that more accurate fluid modelling of the ions as a fluid would bring fluid code results more in line with the experimental results. As the complex nature of the model equations makes any qualitative assessment of this question nearly impossible, computer experiments with codes more like the one described here should be performed to clarify which mechanisms are dominant. 
We now proceed to detail the fluid approximation that will be implemented into the computer simulation code. The plasma is assumed to be composed of electrons, deuterium ions, deuterium atoms, and deuterium molecules; no impurity species are included in the analysis. To start with, expressions are needed for the number densities of the four species in terms of the level of dissociation and the level of ionization. Consider the four species system in the fictitious situation where no ionization or dissociation has occurred. Then if $n_{0}$ is the total number of particles in the system. we have

$$
n_{0}=n_{D_{2}}
$$

Assume next that a fraction $f_{d}$ of these molecules are dissociated. This gives

$$
n_{D_{2}}=n_{0}-f_{d} n_{0}=n_{0}\left(1-f_{d}\right)
$$

and

$$
n_{D}=2 f_{d} n_{0}
$$

Finally, suppose that a fraction $f_{i}$ of the total number of atoms in the system (counting those in molecules) are ionized. This leaves the number of molecules unchanged. If all of the ions are in the same charge state, i.e., $Z$-fold ionized. then 


$$
n_{D}=f_{d}\left(2 n_{0}\right)-f_{1}\left(2 n_{0}\right)=2 n_{o}\left(f_{d}-f_{1}\right) .
$$

$$
n_{i}=2 n_{0} f_{i}
$$

and

$$
n_{e}=2 n_{0} f_{i} Z
$$

Now the one-fluid density is defined to be

$$
\rho=\sum_{\alpha} n_{a} m_{\alpha}
$$

In this context, neglecting terms of order $\left(m_{\mathrm{p}} / m_{\mathrm{i}}\right)$ and assuming the ions and atoms have the sanne mass gives

$$
\rho=2 m_{i} n_{a}\left[1+\frac{Z f_{i} m_{e}}{m_{i}}\right] \approx z m_{i} n_{o}
$$

Or

$$
n_{0} \approx \frac{\rho}{2 m_{i}}
$$




$$
n_{D_{z}} \approx \frac{\rho\left(1-f_{d}\right)}{2 m_{i}}
$$

$$
n_{D} \approx \frac{\rho\left(f_{d}-f_{i}\right)}{m_{i}}
$$

$$
n_{\imath} \approx \frac{\rho f_{i}}{m_{i}}
$$

and

$$
n_{e} \approx \frac{Z p f_{i}}{m_{i}}
$$

Note that this last equation is really a statement of quasineutrality. i.e.. $n_{e}=Z n_{i}$

With expressions for the number densities in terms of the fluid density, we are now prepared to consider the fluid equations. Derivation of the fluid equations involves taking moments of the plasma kinetic equations, ${ }^{64}$ 


$$
\frac{\partial f_{a}}{\partial t}+\boldsymbol{v} \cdot \nabla f_{a}-\frac{2 \underline{\alpha}}{m_{\alpha}}\left[\boldsymbol{E}+\frac{\boldsymbol{v} \times \boldsymbol{B}}{c}\right] \nabla_{\boldsymbol{v}} f_{\alpha}=C_{\alpha} .
$$

where $z_{a}$ is the charge state of the $\alpha^{t h}$ species, and the collision term is given by

$$
C_{\alpha}=\sum_{\beta} C_{\alpha \beta}
$$

Several moments of the distribution function $f_{a}$ arise in finding the fluid

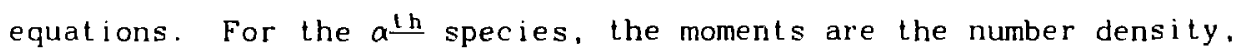

$$
n_{\alpha}(x, t)=\int f_{\alpha}(x, \mathbf{v}, t) d^{3} v
$$

the average velocity of the particles,

$$
\boldsymbol{v}_{\alpha}(x, t)=\frac{1}{n_{\alpha}} \int \boldsymbol{v} f_{a}(x, v, t) d^{3} v
$$

the scalar pressure,

$$
p_{a}(x, t)=\frac{m_{a}}{3} \int\left(v-v_{a}(x, t)\right) \cdot\left(\mathbf{v}-v_{a}(x, t)\right) f_{a}(x, v, t) d^{3} v
$$

the pressure tensor ( $\pi_{a}$ is the stress tensor). 


$$
P_{\alpha}(x, t)=m_{\alpha} \int\left(v-v_{\alpha}(x, t)\right)\left(v-v_{a}(x, t)\right) f_{\alpha}(x, v, t) a^{3} v=p_{\alpha} t+\pi_{\alpha}
$$

the frictional momentum force between the $a^{\underline{t h}}$ and all other species,

$$
\boldsymbol{R}_{a}(x, t)=m_{\alpha} \int\left(\mathbf{v}-\mathbf{v}_{\alpha}(x, t)\right) c_{a} f_{a}(x, \mathbf{v}, t) d^{3} v
$$

the temperature,

$$
T_{\alpha}(x, t)=\frac{m_{\alpha}}{3 n_{\alpha}} \int\left(\mathbf{v}-\mathbf{v}_{\alpha}(x, t)\right) \cdot\left(\mathbf{v}-\mathbf{v}_{\alpha}(x, t)\right) f_{\alpha}(x, v, t) d^{3} v
$$

the heat flux vector,

$$
\mathbf{q}_{\alpha}(x, t)=\frac{m_{\alpha}}{2} \int\left(v-\mathbf{v}_{\alpha}(x, t)\right) \cdot\left(\mathbf{v}-\mathbf{v}_{\alpha}(x, t)\right) v f_{\alpha}(x, v, t) d^{3} v
$$

and the heat transfer from all other species to the $\alpha$ th species,

$$
Q_{a}(x, t)=\frac{m_{a}}{2} \int\left(v-v_{a}(x, t)\right) \cdot\left(v-v_{a}(x, t)\right) C_{a} f_{a}(x, v, t) d^{3} v
$$

Integrating the kinetic equations, (2.17), over velocity space, i.e.. taking the zeroth moment. ${ }^{105}$ yields the continuity equations, 


$$
\frac{\partial n_{\alpha}}{\partial t}+\nabla \cdot\left(n_{\alpha} \mathbf{v}_{\alpha}\right)=0
$$

The momenta equations come from taking the first moment ${ }^{105}$ of equations (2.17):

$$
\begin{aligned}
m_{a} n_{a}\left[\frac{\partial \boldsymbol{v}_{a}}{\partial t}\right. & \left.+\left(\boldsymbol{v}_{a} \cdot \nabla\right) \boldsymbol{v}_{a}\right]= \\
& -\nabla p_{a}-\nabla \cdot \pi_{a}+z_{a} e n_{a}\left[\mathbf{E}+\frac{\boldsymbol{v}_{a} \times \boldsymbol{B}}{c}\right]+\boldsymbol{A}_{\alpha}
\end{aligned}
$$

Finally, the second moment of equations (2.17), found by multiplying by $v \cdot v$ and integrating over velocity space. ${ }^{105}$ gives the energy equations,

$$
\frac{3}{2} n_{a}\left[\frac{\partial T_{a}}{\partial t}+\mathbf{v}_{a} \cdot \nabla T_{a}\right]+p_{a} \nabla \cdot \mathbf{v}_{a}=-\nabla \cdot q_{a}-\pi_{a}: \nabla \mathbf{v}_{a}+Q_{a}
$$

Quantities which are going to be useful in obtaining a reduced description of the plasma are equation (2.10), the one-fluid velocity

$$
v=\frac{\sum_{a} m_{\alpha} n_{a} v_{\alpha}}{\rho},
$$

and the current density 


$$
J=\sum_{\alpha} z_{a} n_{\alpha} \mathbf{v}_{\alpha}
$$

For the deuterium plasma under consideration, the density and the center-of-mass velocity become

$$
\rho=n_{\mathrm{e}} m_{\mathrm{e}}+n_{i} m_{i}+n_{D} m_{D}+n_{D_{2}} m_{D_{2}}
$$

and

$$
\mathbf{v}=\rho^{-1}\left(n_{e} m_{e} \mathbf{v}_{e}+n_{i} m_{i} \mathbf{v}_{i}+n_{D} m_{D} \mathbf{v}_{D}+n_{D_{2}} m_{D_{z}} \mathbf{v}_{D_{z}}\right)
$$

In a deuterium plasma where all the ions have been $Z$-fold ionized, the current density becomes

$$
\boldsymbol{J}=\operatorname{Zen}_{i}\left(\mathbf{v}_{i}-\mathbf{v}_{e}\right)
$$

One major simplifying assumption is now invoked. It is to assume that there is no slip between the fluids of deuterium molecules, deuterium atoms, and deuterium ions:

$$
v_{i}=v_{D}=v_{D_{2}}
$$

With this approximation, $(2.30)$ becomes: 


$$
\mathbf{v}=\frac{n_{e} m_{e} \mathbf{v}_{e}+n_{h} m_{i} \mathbf{v}_{2}}{\rho}
$$

where

$$
n_{h}=n_{2}+n_{D}+n_{D_{2}} \approx \frac{\rho}{m_{2}} .
$$

Solving the $\boldsymbol{v}$ and $\boldsymbol{J}$ equations for $\boldsymbol{v}_{\mathfrak{z}}$ and $\boldsymbol{v}_{\boldsymbol{e}}$ yields

$$
v_{e}=v-\frac{m_{i} n_{h}}{e n_{e} \rho} \approx \mathbf{v}-\frac{1+f_{d}}{2 e n_{e}} \boldsymbol{J} \approx \mathbf{v}-\frac{1}{e n_{e}} \boldsymbol{d}
$$

and

$$
v_{2}=v+\frac{m_{e}}{e \rho} \approx v
$$

Here we have used $f_{d} \approx 1$ since the ionized state which the $\mathbf{v}_{\mathbf{p}}$ equation describes will not be appreciably populated until $f_{d}$ is essentially unity. Noting in advance the definition (2.41), this gives the relative velocity, $u$, as

$$
u=-\frac{1}{e n_{e}} \boldsymbol{J}
$$

This set of moment equations contains a third-order velocity moment of $f_{a}, \mathbf{q}_{a}$. 
which must be found by solving a kinetic equation for $f_{a}$ as there is no equation defining it. Alternatively, one can make simplifying assumptions about the form of $f_{\alpha}$ to obtain analytic results. Similarly, $\boldsymbol{A}_{\alpha}$ and $Q_{\alpha}$ must be found by performing integrals over velocity space involving collision terms. Braginski i performs calculations for distribution functions which are close to Maxwellians and collision terms in the Landau form (see (4.3) in Braginski ${ }^{9}$ ). In certain situations such as regimes where microturbulence is important, the assumption that these distribution functions are nearly Maxwellian is a poor one. However. under most plasma conditions in medium density, high- $\beta$ pinches, the distribution functions should be fairly close to a Maxwellian so the equations that follow are an important first approximation in any event. As the relative velocity, $u$, appears in these results, we def ine it here:

$$
u \equiv \mathbf{v}_{e}-\mathbf{v}_{i}
$$

Further, we define

$$
\boldsymbol{A} \equiv \boldsymbol{R}_{\mathrm{e}}=\boldsymbol{R}_{e i}+\boldsymbol{R}_{e n}
$$

Since there is no slip between ions and neutrals, there should be no momentum transfer between them. Hence.

$$
\boldsymbol{R}_{i}=\boldsymbol{R}_{i e}+\boldsymbol{R}_{i n} \approx-\boldsymbol{R}_{e i} .
$$




$$
\boldsymbol{A}_{D}+\boldsymbol{R}_{D_{2}} \equiv \boldsymbol{R}_{n}=\boldsymbol{R}_{n e}+\boldsymbol{R}_{n i} \approx-\boldsymbol{R}_{e n} .
$$

so that

$$
\boldsymbol{R}_{i}+\boldsymbol{R}_{D}+\boldsymbol{R}_{D_{2}}=-\boldsymbol{R}
$$

Using this definition the appropriate expressions are: ${ }^{9}$

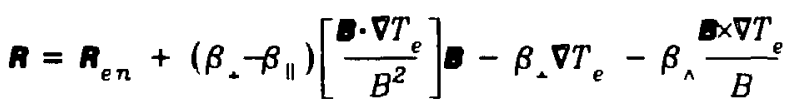

$$
\begin{aligned}
& +\left(\alpha_{+}-\alpha_{\| 1}\right)\left[\frac{B \cdot u}{B^{2}}\right] \cdot-\alpha_{+} u+\alpha_{A} \frac{B x u}{B} \text {. }
\end{aligned}
$$

$$
\begin{aligned}
& \mathbf{q}_{\mathrm{e}}=\left(\kappa_{+}^{e}-\kappa_{\|}^{e}\right)\left[\frac{\boldsymbol{E} \cdot \nabla T_{e}}{B^{2}}\right] \cdot-\kappa_{+}^{e} \nabla T_{e}-\kappa_{\wedge}^{e} \frac{\Delta \times \nabla T_{e}}{B}
\end{aligned}
$$

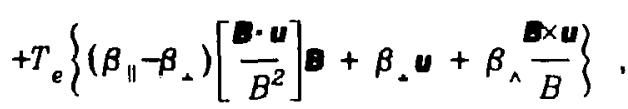




$$
\mathbf{q}_{i}=\left(\kappa_{-}^{i}-\kappa_{\|}^{i}\right)\left[\frac{\boldsymbol{B} \cdot \nabla^{\prime} T_{i}}{B^{2}}\right] \boldsymbol{B}-\kappa_{\perp}^{i} \nabla T_{i}+\kappa_{\wedge}^{i} \frac{\boldsymbol{B} \nabla T_{i}}{B}
$$

$$
Q_{e}=-Q_{i}-\boldsymbol{R} \cdot \boldsymbol{u} .
$$

and

$$
Q_{i}=\frac{3 n_{e} m_{e}\left(T_{e}-T_{i}\right)}{\tau_{e} m_{i}}
$$

$\boldsymbol{R}_{e n}$ is given by ${ }^{9}$ ?

$$
\boldsymbol{R}_{e n}=\left(\frac{e Z \rho f_{i}}{m_{i}}\right)^{2} \eta_{n} \boldsymbol{u}
$$

The transport coefficients in equations (2.46) through (2.51) are:

$$
\eta_{n}=\frac{1-f_{i}}{e^{2} Z f_{i}} \sigma_{n e} \sqrt{\frac{8 m_{e} T_{e}}{\pi}}
$$

$$
\alpha_{\|}=\frac{\alpha_{0} m_{e} n_{e}}{\tau_{e}} \text {. }
$$

$-40-$ 


$$
a_{\perp}=\frac{m_{e} n_{e}}{T_{e}}\left\{1-\frac{\alpha_{1} x_{e}^{2}+\alpha_{o}}{\Delta_{e}}\right\},
$$

$$
\alpha_{\Lambda}=\frac{m_{e} n_{e}}{\tau_{e}} \frac{x_{e}\left(a_{i}, x_{e}^{2}+\alpha_{o}^{\prime \prime}\right)}{\Delta_{e}} .
$$

$$
\beta_{\|}=\beta_{o} n_{e},
$$

$$
\beta_{+}=n_{\mathrm{e}} \frac{\beta_{i}^{\prime} x_{\mathrm{e}}^{2}+\beta_{0}}{\Delta_{\mathrm{e}}},
$$

$$
\beta_{n}=n_{e} x_{e} \frac{\beta_{,}^{\prime \prime} x_{e}^{2}+\beta_{0}^{\prime \prime}}{\Delta_{e}} .
$$




$$
\kappa_{\|}^{e}=\gamma_{0} \frac{n_{e} T_{e} \tau_{e}}{m_{e}},
$$

$$
\kappa_{+}^{e}=\frac{n_{e} T_{e} \tau_{e}}{m_{e}} \frac{\gamma_{i}^{\prime} x_{e}^{2}+\gamma_{j}^{\prime}}{\Delta_{e}},
$$

$$
\kappa_{\hat{\Lambda}}^{e}=\frac{n_{e} T_{e} \tau_{e} x_{e}}{m_{e}} \frac{\gamma_{,}^{\prime \prime} x_{e}^{2}+\gamma_{o}^{\prime \prime}}{\Delta_{e}}
$$

$$
\kappa_{\|}^{i}=\xi_{0} \frac{n_{i} T_{i} \tau_{i}}{m_{i}} \text {, }
$$

$$
\kappa_{i}^{i}=\frac{n_{i} T_{i} \tau_{i}}{m_{i}} \frac{\xi_{t}^{\prime} x_{i}^{2}+\xi_{j}}{\Delta_{i}},
$$




$$
\kappa_{A}^{2}=\frac{n_{i} T_{2} \tau_{i} x_{2}}{m_{i}} \frac{\xi_{1} x_{2}^{2}+\xi_{0}^{\prime \prime}}{\Delta_{i}},
$$

where

$$
\begin{gathered}
\Delta_{e}=x_{e}^{2}\left(x_{e}^{2}+\delta_{1}^{e}\right)+\delta_{0}^{e}, \\
x_{e}=\omega_{e} \tau_{e}, \\
\omega_{e}=\frac{e B}{m_{e} c} .
\end{gathered}
$$

$$
\tau_{e}=\frac{3 \sqrt{e} T_{e}^{3 / 2}}{4 \sqrt{ }(2 \pi) e^{4} n_{i} Z^{2} \log \Lambda_{e}}
$$

$$
\Delta_{i}=x_{i}^{2}\left(x_{i}^{2}+\delta_{i}^{i}\right)+\delta_{0}^{i} .
$$




$$
x_{i}=\omega_{i} \tau_{i}
$$

$$
\omega_{i}=\frac{Z e B}{m_{i} c} \text {, }
$$

$$
\tau_{i}=\frac{3 \sqrt{m_{i}} T_{i}^{3 / 2}}{4 \sqrt{ } \pi e^{4} n_{i} Z^{4} \log \Lambda_{i}}
$$

$$
\log \Lambda_{e}=\left\{\begin{array}{l}
C_{Q N}^{e}+\frac{1}{2} \log \left\{\begin{array}{l}
T_{e}^{2} \\
n_{e}
\end{array}\right\}, C_{Q N}^{e}-C_{C L S}^{e}-\frac{1}{2} \log \left\{\frac{T_{e}}{Z^{2}}\right\}<0 \\
C_{C L S}^{e}+\frac{1}{2} \log \left\{\frac{T_{e}^{3}}{Z^{2} n_{e}}\right\}, C_{Q N}^{e}-C_{C L S}^{e}-\frac{1}{2} \log \left\{\frac{T_{e}}{Z^{2}}\right\}>0
\end{array},\right.
$$

and

$$
\log \Lambda_{i}=\left\{\begin{array}{l}
C_{Q M}^{i}+\frac{1}{2} \log \left\{\frac{A T_{e} T_{i}}{n_{e}}\right\}, C_{Q M}^{i}-C_{C L S}^{i}+\frac{1}{2} \log \left\{\frac{A Z^{4}}{T_{i}}\right\}<0 \\
C_{C L S}^{i}+\frac{1}{2} \log \left\{\frac{T_{e} T_{i}^{2}}{Z^{4} n_{e}}\right\}, C_{Q M}^{i}-C_{C L S}^{i}+\frac{1}{2} \log \left\{\frac{A Z^{4}}{T_{i}}\right\}>0
\end{array} .\right.
$$

Braginskii ${ }^{9}$ gives values for $\alpha_{0}, \alpha_{0}, a_{1}^{\prime}, \alpha_{0}^{\prime \prime}, \alpha_{1}^{\prime \prime}, \beta_{0}, \beta_{0}^{\prime}, \beta_{,}^{\prime}, \beta_{0}^{\prime \prime}, \beta_{1}^{\prime \prime}, \gamma_{0}, \gamma_{0}, \gamma_{1}$, 


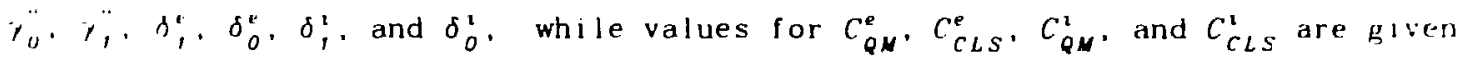
by chandler. 18

Proceeding to reduce the multispecies transport equations, we begin with the number density equations (2.27). Simply multiplying by $m_{a}$ and adding the resulting equations yields:

$$
\frac{\partial \rho}{\partial t}+\nabla \cdot(\rho \boldsymbol{\nu})=0
$$

To obtain the one-fluid momentum equation, we begin by adding the momenta equations. (2.28). Using (2.38) and (2.39), each momentum equation involves terms like

$$
[(\mathbf{r}+g \mathbf{J}) \cdot \nabla](\mathbf{r}+g \boldsymbol{J})
$$

Using the result of Appendix A, (2.76) can be written

$$
\begin{aligned}
& {[(\boldsymbol{v}+g \boldsymbol{J}) \cdot \nabla](v+g \boldsymbol{J})=} \\
& (\boldsymbol{v} \cdot \nabla) v+(J \cdot \nabla) v+J(v \cdot \nabla g)+g(v \cdot \nabla) J+g J(J \cdot \nabla g)+g^{2}(J \cdot \nabla) J .
\end{aligned}
$$

Noting in addition that

$$
\boldsymbol{n}_{e}+\boldsymbol{R}_{i}+\boldsymbol{R}_{D}+\boldsymbol{A}_{D_{2}}=0
$$


and assuming quasineutrality $\left(Z n_{\imath}=n_{e}\right)$ gives the following result for the one-f luid momentum equation:

$$
\begin{aligned}
\rho\left[\frac{\partial \mathbf{v}}{\partial t}+(\boldsymbol{v} \cdot \nabla) \mathbf{v}\right] & +\frac{m_{e} m_{i}}{e_{e} \rho}\left[n_{h} \frac{\partial n_{e}}{\partial t}-n_{e} \frac{\partial n_{h}}{\partial t}\right] \\
+ & \frac{m_{e} m_{i} n_{h}}{e^{2} \rho}\left\{\frac{m_{i}}{n_{e}^{2} \rho}(\boldsymbol{J}-\boldsymbol{v}) \cdot\left(n_{e} \nabla n_{h}-n_{h} \nabla_{e}\right)+\nabla \cdot(\boldsymbol{J})\right. \\
& \left.+\frac{m_{i} n_{h}}{\rho^{2} n_{e}}\left[\left(1-\frac{m_{e} n_{e}}{m_{i} n_{h}}\right)(\boldsymbol{v} \cdot \nabla \rho)-\left(1+\frac{m_{e} n_{e}}{m_{i} n_{h}}\right)(\boldsymbol{J} \cdot \nabla \rho)\right]\right\}= \\
& -\nabla\left(p_{e}+p_{h}\right)+\frac{\mathbf{J} \times \mathbf{B}}{c}-\nabla \cdot\left[\sum_{a} \pi_{a}\right] .
\end{aligned}
$$

For $T_{i} \sim T_{e}$, the largest contribution to $\Sigma_{a} \pi_{a}$ is $\pi_{i}$, so $\nabla \cdot \pi_{i}$ is the term to estimate. In the absence of a magnetic field, equations (2.19), (2.20), and (2.22) of Braginskii ${ }^{9}$ give

$$
\left(\boldsymbol{\pi}_{i}\right)_{j k}=-.96 n_{i} T_{i} \tau_{i}\left[\frac{\partial\left(\mathbf{v}_{i}\right)_{j}}{\partial x_{k}}+\frac{\partial\left(\mathbf{v}_{i}\right)_{k}}{\partial x_{j}}-\frac{2}{3} \delta_{j k} \nabla \cdot \boldsymbol{v}_{i}\right]
$$

Since the magnetic field reduces the of $f-d i a g o n a l$ teris by $(\omega \tau)^{-1}$ or $(\omega \tau)^{-2}$, the diagonal terms are dominant. Therefore, the appropriate scaling is

$$
\left|\nabla \cdot \pi_{i}\right| \sim \frac{p_{i}}{L} \frac{T_{i}|\boldsymbol{v}|}{L}
$$

Next, compare this against the gradient of the scalar pressure 


$$
\left|\nabla p_{i}\right| \sim \frac{p_{2}}{L}
$$

It is evident that for $\nabla \cdot \pi_{i}$ to be negligible, $\lambda_{i} \sim \tau_{i}|r|<<L$ must hold. This will be the case in colder, more dense pinch-like plasmas. Contrariwise, it appears that there are high-temperature. low-density regimes where this effect is not negligible, such as could occur during preionization. However, I ignore the effect here.

Next noting that

$$
\left|\nabla\left(\frac{m_{e}}{e \rho}\right)\right| \sim \frac{m_{e}}{e \rho L}
$$

is snall in the $\left(m_{e} / m_{2}\right)$ mass ratio, and further, noting that dividing equation (2.79) by $\rho$ makes a number of other terms small in that same ratio, the final momentum equation becomes

$$
\rho\left[\frac{\partial \mathbf{v}}{\partial t}+(\mathbf{v} \cdot \nabla) \mathbf{v}\right]+\nabla\left(p_{e}+p_{h}\right)-\frac{J \times \bullet}{c}=0
$$

The last moment equation is that for the temperature. Consider first the heavy particles. In conjunction with the assumption of no slip between these fluids, it is further assumed that they are at the same temperature; i.e., 
$T_{D}=T_{i}$ and $T_{D_{2}}=T_{2}$. Adding the neutral species and ion energy equations then yi $\in \operatorname{lds}$

$$
\begin{aligned}
\frac{3}{2}\left(n_{i}+n_{D}+n_{D_{2}}\right) & {\left[\frac{\partial T_{i}}{\partial t}+\mathbf{v}_{i} \cdot \nabla T_{i}\right]+p_{h} \boldsymbol{\nabla} \cdot \mathbf{v}_{i}=} \\
& -\nabla \cdot\left(\boldsymbol{q}_{i}+\mathbf{q}_{D}+\mathbf{q}_{D_{2}}\right)+Q_{i}+Q_{D}+Q_{D_{2}}-\sum_{a} \pi_{a}: \nabla \mathbf{v}_{i} .
\end{aligned}
$$

As the term involving the viscous stress tensor scales as given in (2.81) times $\left|\mathbf{v}_{i}\right|$, and as

$$
\left|p_{h} \boldsymbol{\nabla} \cdot \mathbf{v}_{i}\right| \sim \frac{p_{h}}{L}\left|\mathbf{v}_{i}\right|
$$

the same argument concerning the validity of the ordering $\lambda_{i} \sim \tau_{i}|v|<<L$ applies here. Therefore, the viscous terms can be neglected. It is also assumed that there is negligible heat transfer to the neutral species. Thus, using (2.39), the heavy particle energy equation simplifies to

$$
\frac{3\left(1+f_{d}\right) \rho}{4 m_{i}}\left(\frac{\partial T_{i}}{\partial t}+\mathbf{v} \cdot \nabla T_{i}\right)+p_{h} \nabla \cdot \mathbf{v}=-\nabla \cdot \boldsymbol{q}_{i}+Q_{i}
$$

The electron energy equation is 


$$
\frac{3 n_{e}}{2}\left[\frac{\partial T_{e}}{\partial t}+\mathbf{v}_{e} \cdot \nabla T_{e}\right]+p_{e} \nabla \cdot \mathbf{v}_{e}=-\nabla \cdot q_{e}-\pi_{e}: \nabla \boldsymbol{V}_{e}+Q_{e} .
$$

Because the viscous stress tensor term has order of magnitude

$$
\left|\pi_{e}: \nabla \mathbf{v}_{e}\right| \sim \frac{n_{e} T_{e} \tau_{e}\left|\mathbf{v}_{e}\right|}{L} \frac{\left|\mathbf{v}_{e}\right|}{L}=\frac{p_{e} \tau_{e}\left|\mathbf{V}_{e}\right|^{2}}{L^{2}},
$$

and the pressure term goes as

$$
p_{e}\left|\nabla \cdot \boldsymbol{v}_{e}\right| \sim \frac{p_{e}\left|\mathbf{v}_{\varepsilon}\right|}{L}
$$

the viscous effects can be ignored if $\lambda_{e} \sim \tau_{e}\left|\boldsymbol{v}_{e}\right|<<L$. This should hold under plasma conditions of interest here. To account for radiation losses, an explicitly calculated term is subtracted from the energy equation:

$$
\begin{aligned}
\frac{3 Z f_{i} \rho}{Z m_{i}}\left[\frac{\partial T_{e}}{\partial t}+\left(\mathbf{v}-\frac{m_{i}}{Z e \rho f_{i}} \boldsymbol{J}\right) \cdot \nabla T_{e}\right]+p_{e} \boldsymbol{\nabla} \cdot\left(\mathbf{v}-\frac{m_{i}}{Z e \rho f_{i}} \boldsymbol{J}\right)= \\
-\boldsymbol{\nabla} \cdot \mathbf{q}_{e}-Q_{i}-\boldsymbol{R} \cdot \boldsymbol{u}-c_{b r e m} G_{f} \rho^{2} v T_{e} .
\end{aligned}
$$

Here $G_{f}$ is the sum of Gaunt factors ${ }^{91}$ or collision strengths for bound-bound, bound-free, and Iine radiation mechanisms in a hydrogen-like plasma. ${ }^{\gamma 2}$ lt includes the effect of plasma screening on the hydrogenic energy levels.98 
Yet another equation can be found from the moment equations. To obtain this equation which is a generalized $\mathrm{Ohm}^{\prime} \mathrm{s}$ law, multiply the momentum equation for the $\alpha^{\frac{t h}{h}}$ species, $(2.28)$, by $\left(z_{a} e / m_{a}\right)$ and add all of the resulting equations. By using the continuity equation for $n_{e}$.

$$
\frac{\partial n_{e}}{\partial t}=-\nabla \cdot\left[n_{e}\left(v-\frac{m_{i} n_{h}}{e n_{e} \rho}\right)\right]
$$

the vector identity $C-18$ in Stacey, 108

$$
\boldsymbol{\nabla} \cdot(\boldsymbol{a b})=(\boldsymbol{\nabla} \cdot \mathbf{a}) \mathbf{b}+(\mathbf{a} \cdot \boldsymbol{\nabla}) \mathbf{b}
$$

and the vector form of quasineutrality,

$$
\boldsymbol{\nabla} \cdot \boldsymbol{J}=0
$$

we obtain 


$$
\begin{aligned}
& \frac{\partial \boldsymbol{J}}{\partial t}+\nabla \cdot(\boldsymbol{J} \boldsymbol{r}+\boldsymbol{\gamma} \boldsymbol{J})+\frac{m_{\epsilon} n_{\epsilon}-m_{\boldsymbol{\imath}} n_{h}}{\epsilon n_{\epsilon} \rho} \nabla \cdot(\boldsymbol{J} \boldsymbol{J}) \\
& +\int \frac{m_{i}^{2} n_{h}^{2}}{\epsilon n_{e} \rho^{3}}\left[1+\frac{\rho}{m_{i} n_{h}}-\left(\frac{m_{e} n_{e}}{m_{i} n_{h}}\right)^{2}\right](\boldsymbol{J} \cdot \nabla \rho)-\frac{m_{2}}{e n_{e} \rho}\left(1+\frac{m_{i} n_{h}}{\rho}\right)\left(\boldsymbol{J} \cdot \nabla n_{h}\right) \\
& \left.+\frac{m_{2} n_{h}}{\epsilon \rho n_{e}^{2}}\left(1+\frac{m_{2} n_{h}}{\rho}\right)\left(J \cdot \nabla n_{e}\right)\right\}= \\
& \frac{\epsilon^{2} n_{e}}{m_{E}}\left(1+\frac{Z m_{e}}{m_{i}}\right)\left[\boldsymbol{E}+\frac{\mathbf{v} \times \boldsymbol{\theta}}{c}\right]-\frac{e m_{2} n_{h}}{m_{\mathrm{e}} \rho}\left[1-\frac{Z n_{\mathrm{e}} m_{e}^{2}}{n_{h} m_{i}^{2}}\right] \frac{\boldsymbol{J} \times \boldsymbol{\theta}}{c} \\
& -\frac{Z e}{m_{i}}\left[\nabla \cdot \pi_{i}+\nabla p_{i}\right]+\frac{e}{m_{e}}\left[\nabla \cdot \pi_{e}+\nabla p_{e}\right]+\frac{Z e}{m_{i}}\left(\boldsymbol{R}_{e n}-\boldsymbol{R}\right)+\frac{e}{m_{e}}-\boldsymbol{R} .
\end{aligned}
$$

Using approximate values for $n_{e}$ and $n_{h}$, neglecting terms small in $\left(m_{e} / m_{1}\right)$, and solving for E. (2.95) becomes

$$
\begin{aligned}
\boldsymbol{E}= & \frac{m_{e} m_{i}}{Z e^{2} \rho f_{i}}\left[\frac{\partial \boldsymbol{J}}{\partial t}+\nabla \cdot(\boldsymbol{J}+\boldsymbol{J})\right]-\frac{m_{e} m_{i}^{2}}{Z^{2} e^{3} \rho^{2} f_{i}^{2}} \nabla \cdot(\boldsymbol{J}) \\
& +\frac{Z m_{e} m_{i}^{2}}{Z^{2} e^{3} \rho^{3} f_{i}^{3}}\left[f_{i}(\boldsymbol{J} \cdot \nabla \rho)+\rho\left(\boldsymbol{J} \cdot \nabla f_{i}\right)\right] J \\
& -\frac{\boldsymbol{\nabla} \times \boldsymbol{B}}{c}+\frac{m_{i}}{Z e \rho f_{i}}\left[\frac{\boldsymbol{J} \times \boldsymbol{B}}{c}-\nabla \cdot \pi_{e}-\nabla p_{e}-\boldsymbol{n}\right] .
\end{aligned}
$$

By using the same reasoning that led to ignoring the electron viscous effects in the electron energy equation, it is obvious these effects can be neglected here as well. To estimate the order of magnitude of $|\mu|$, we use Ampere's law: 


$$
|\lambda| \sim \frac{C}{4 \pi} \frac{B}{L}
$$

The order of magnitude of various terms in (2.96) can be estimated as follows:

$$
\left|\frac{\partial J}{\partial t}\right| \sim \frac{c B \omega}{4 \pi L}
$$

$$
|\nabla \cdot(J v+v J)| \sim \frac{c B|v|}{4 \pi L}
$$

and

$$
\left|\mathcal{L}\left[f_{i}(J \cdot \nabla \rho)+\rho\left(\mathcal{J} \cdot \nabla f_{i}\right)\right]\right| \sim \frac{c^{2} B^{2} \rho f_{i}}{16 \pi^{2} L^{3}}
$$

A reference term for this equation is the $\times$ term: 


$$
\begin{aligned}
\left|\frac{Z e^{2} \rho f_{2}}{m_{i} m_{e} c} \times \boldsymbol{B}\right| & \sim \frac{e^{2} n_{e}|\mathbf{r}| B}{m_{e} c} \\
& =\left(\frac{4 \pi m_{e} e^{2}}{m_{e}}\right)\left(\frac{L}{c}\right)\left(\frac{|\mathbf{v}|}{c}\right)\left(\frac{c B}{4 \pi L}\right) \\
& =\omega_{p e}\left(\frac{L}{\delta}\right)\left(\frac{|v|}{c}\right)\left(\frac{c B}{4 \pi L}\right) .
\end{aligned}
$$

This estimate implies $\omega_{p e}(L / \delta)(|\nabla| / c) \gg \omega$ is necessary in order to neglect the time derivative of $\boldsymbol{J}$. This is easily satisfied in most pinch experiments, the present Topolotron included. Via a slight rearrangement, (2.101) can be written:

$$
\left|\frac{Z e^{2} \rho f_{i}}{m_{\mathrm{e}} m_{i} c} \times \Delta\right| \sim \frac{L^{2}}{\delta^{2}}\left(\frac{|v|}{L}\right)\left(\frac{c B}{4 \pi L}\right) .
$$

so that $(L / \delta)^{2} \gg$, implies the divergence of $(v d+d v)$ is small. For comparison with (2.100), a new multiple of the $\forall \times$ term is required:

$$
\begin{aligned}
\left|\frac{Z^{2} e^{3} \rho^{3} f_{i}^{3}}{2 m_{i}^{2} m_{e} c} v \times\right| & \sim \frac{n_{e}^{2} e^{3} \rho f_{i}}{m_{e} c} B|\mathbf{v}| \\
& =\left(\frac{16 \pi^{2} n_{e}^{2} e^{4}}{m_{e}^{2}}\right)\left(\frac{L^{3}}{c^{3}}\right)\left(\frac{m_{e} c}{e B}\right)\left(\frac{|v|}{c}\right)\left(\frac{c^{2} B^{2} \rho f_{i}}{16 \pi^{2} L^{3}}\right) \\
& =\frac{\omega_{p e}^{4} L^{3}}{c^{3}}\left(\frac{m_{e} c}{e B}\right)\left(\frac{|v|}{c}\right)\left(\frac{c^{2} B^{2} \rho f_{i}}{16 \pi^{2} L^{3}}\right) \\
& =\frac{\omega_{p e}\left(\frac{L}{\delta}\right)^{3}\left(\frac{|v|}{c}\right)\left(\frac{c^{2} B^{2} p f_{i}}{16 \pi^{2} L^{3}}\right) .}{\omega_{c e}} .
\end{aligned}
$$


Since $\omega_{p e} / \omega_{c e} \geqslant 100$, this term will be smaller than (2.99) in all cases, and is therefore dropped. The generalized $\mathrm{Ohm}^{\prime} \mathrm{s}$ law, (2.96), is f inally

$$
E=-\frac{\mathbf{v} \times \boldsymbol{B}}{c}-\frac{m_{i}}{Z e \rho f_{i}}\left[\nabla p_{e}+\boldsymbol{A}-\frac{\boldsymbol{J} \times \boldsymbol{B}}{c}\right]
$$

Since the total magnetic field is the sum of plasma and external field contributions, $\theta=\nabla^{p}+\theta^{e x t}$, equation (2.104) can be used to convert Faraday's law into

$$
\frac{\partial \mathscr{E}^{p}}{\partial t}=-\frac{\partial \boldsymbol{B}^{e x t}}{\partial t}+\nabla \times\left\{\mathbf{V} \times \boldsymbol{B}+\left(\frac{m_{i}}{Z e \rho f_{i}}\right)\left[c\left(\boldsymbol{A}+\nabla p_{e}\right)-\boldsymbol{J} \times \boldsymbol{B}\right]\right\}
$$

While (2.104) eliminates E from the system, there are still two Maxwell equations which are needed in the model. First is the constraint of no magnetic monopoles described by

$$
\boldsymbol{\nabla} \cdot \boldsymbol{B}=0
$$

and second is Ampere's law,

$$
\nabla \times B=\frac{4 \pi}{c} J+\frac{1}{c} \frac{\partial E}{\partial t}
$$

The left-hand side scales 1 ike $B / L$, and the displacement current like 


$$
\left|\begin{array}{ll}
\frac{1}{c} & \frac{\partial E}{\partial t}
\end{array}\right| \sim \frac{\omega E}{c} \sim \frac{\omega \mid \mathbf{v | B}}{c^{2}} .
$$

So the displacement current may be neglected if

$$
\frac{\omega L}{c} \frac{|v|}{c} \sim\left(\frac{|v|}{c}\right)^{2}
$$

is small. which holds because the plasma is nonrelativistic. Ampere's law therefore reduces to

$$
J=\frac{c}{4 \pi} \nabla \times
$$

and this is used to eliminate from the MHD equations.

It now remains to close the model with appropriate equations of state. The variables which need to be so specified are $p_{e}, p_{h}, f_{i}, f_{d}$. The partial pressures are assumed to follow perfect gas laws. Hence,

$$
p_{e}=n_{e} T_{e} \approx \frac{Z \rho f_{i} T_{e}}{m_{i}}
$$

and 


$$
p_{h}=n_{i} T_{i}+n_{D} T_{D}+n_{D_{2}} T_{D_{z}} \approx \frac{\rho\left(1+f_{d}\right) T_{i}}{2 m_{i}}
$$

For the remaining state variables, there are two possible choices to pursue. Either the plasma is in a local thermodynamic equilibrium (LTE), or it is no: described by any equilibrium model. in the former case. Saha equations are appropriate, while in the latter case, a set of rate equations describing the physical mechanisms driving the dissociation and ionization processes is required. Although an accurate description requires the rate equation solution. at least in the case of ionization. ${ }^{33}$ Saha equation models are nonetheless used in this work.

Starting with the Saha equation for dissociation, we use equation (15) in Nickel ${ }^{89}$

$$
\frac{n_{D}^{2}}{n_{D_{2}}}=\frac{C^{2}}{2^{3 / 2}}\left(\frac{2 \pi m_{i} T_{i}}{h^{2}}\right)^{3 / 2}
$$

where the notation for the number densities is ours. Also, $G$ denotes the number of internal atomic states, $E_{d i s s}$ is the dissociation energy, and $Z$ is the partition function for the molecule with the translational factor removed. This equation is valid only for homonuclear diatomic molecules, but could be modified for other cases by adjustment of the mass in the translational part of (2.113): however. deuterium molecules are of the homonuclear diatomic type. 
Next, we examine the rest of the partition function. It is given by McQuarrie ${ }^{79}$ as

$$
Z=q_{r a t} q_{\nu i b} q_{e l e c} q_{n u c l}
$$

where

$$
q_{r \circ t}=\frac{T_{i}}{2 B},
$$

$$
q_{\nu \mathrm{ib}}=\frac{e^{-h \nu /\left(2 T_{1}\right)}}{1-e^{-h \nu / T_{1}}} .
$$

$$
q_{e l e c}=\omega_{e}, e^{D \cdot / T_{2}}=e^{E_{d, s s} / T_{1}} e^{h \nu /\left(2 T_{1}\right)},
$$

and

$$
q_{n u c l}=(2 l+1)^{2}
$$

In these definitions, we have further defined $B$ to be the rotational constant of the molecule, $h \nu=\omega_{e}$ to be the vibrational constant. $D_{e}$ to be the depth of the 
ground electronic state potential well, and $I$ to be the spin of the nucleii. For ground state deuterium, $I=0$.

In addition to the effects contributing to $Z$ already given, there are coupling effects which modify (2.114) as follows:

$$
Z=\frac{T_{i}}{2 \bar{B}}\left(\frac{e^{E_{d i s} / T_{i}}}{1-e^{-h \nu / T_{i}}}\right)\left\{1+\frac{2 \bar{D} T_{i}}{\bar{B}}+\frac{\alpha}{B\left(e^{h \nu / T_{i}-1}\right)}+\frac{2 h \nu \chi_{e}}{T_{i}\left(e^{h \nu / T_{i}-1}\right)^{2}}\right\}
$$

The corrections are given in terms of $\alpha$, the rotational-vibrational constant, $\chi_{e}$. the anharmonicity constant, and $D$. the centrifugal distortion constant. For deuterium. Huber and Hertzberg ${ }^{51}$ give the following values for these quantities:

$$
E_{\text {diss }}=4.556 \mathrm{eV} \text {. }
$$

$$
\omega_{e}=h \nu=3115.5 \mathrm{~cm}^{-1} \text {, }
$$

$$
\bar{B}=30.443 \mathrm{~cm}^{-1}
$$




$$
\alpha=1.0786 \mathrm{~cm}^{-1} \text {. }
$$

and

$$
\omega_{e} \chi_{e}=h \nu \chi_{e}=61.82 \mathrm{~cm}^{-1} .
$$

Numerical experiments over the entire range of expected values of density and ion temperature showed that the correction terms could be ignored and still maintain $1 \%$ accuracy in the calculation of $f_{d}$. With this in mind. we can rewrite $(2.113)$ as

$$
\left(f_{d}-f_{i}\right)^{2}=\frac{\left(1-f_{d}\right) m_{i}^{5 / 2} \pi^{3 / 2} \sqrt{ } T_{i} \bar{B}\left(1-e^{-h_{i} / T_{1}}\right) e^{-E_{d,}, / T_{2}}}{\rho h^{3}} .
$$

While this quadratic expression for $f_{d}$ is fairly simple, it is unfortunately quadratically coupled to the unknown $f_{i}$. Since $f_{d}$ is only significantly different from unity in a low temperature range where no appreciable ionization has occurred, it is reasonable to accept some minimum value for $f_{\text {, }}$ appropriate to those conditions. A means for computing $f_{i}^{m i n}$ will be given after an equation 
for $f_{2}$ has been developed. With $f_{i}^{m i n}$ known, (2.126) can be solved explicitly for $f_{d}$ in terms of $\rho$ and $\mathrm{T}_{\mathrm{i}}$.

In order to develop a Saha-Boltzmann equation for the ionization process, an equilibrium relation between first and higher excited states in the deuterium atom is needed. This can be written ${ }^{32}$

$$
\frac{n_{D, j}}{n_{D, 1}}=\frac{g_{D, j}}{g_{D, 1}} e^{-\left(E,-E, 1 / T_{E}\right.}
$$

where $g_{D, j}$ is the degeneracy of the $j \underline{t h}$ energy level, $E_{j}$ is its energy, and $n_{D,}$, is the population density of the $j$ th $l e v e l$. Now use the Saha-Eggert equation ${ }^{32}$ for the first excited state, $n_{D, r}$. This gives the population of the $j$ th level as

$$
n_{D, j}=n_{i} n_{e}\left(\frac{g_{D, j}}{2 g_{D}}\right) \frac{h^{3}}{\left(2 \pi m_{e} T_{e}\right)^{3 / 2}} e^{\left(E,-\Delta E_{D}\right) / T},
$$

where $g_{D}=C=$ atomic degeneracy, and $\Delta E_{D}$ is the amount of lowering of the energy levels aue to electric microfields. A more accurate determination of the plasma's affects on the hydrogenic energy levels is obtained by using the results of Rogers, Graboske, and Harwood. ${ }^{88}$ This is noted by treating the energy as $E_{j}\left(\rho, f_{i}\right)$ instead of $E_{j}-\Delta E_{D}$. Now note that 


$$
\sum_{j * 1} n_{D, j}=n_{D}
$$

and

$$
g_{D, j}=2 j^{2}
$$

so that (2.128) becomes

$$
f_{d}-f_{i}=\frac{Z \rho f_{i}^{2}}{m_{i}} \frac{h^{3}}{\left(2 \pi m_{e} T_{e}\right)^{3 / 2}} \sum_{j \geqslant 1} j^{2} e^{E},\left(\rho \cdot f_{i}\right) / T
$$

Once again we have a quadratic equation to solve. And once again, it is coupled to the related variable $f_{d}$. Although the coupling is linear, it does not make sense to solve the coupled equation for $f_{d}$ simultaneously, as that equation contains the assumption that $f_{i}=f_{i}^{m i n}$, which would make the formulation inconsistent. More importantly, whenever any appreciable ionization takes place, the temperature will necessarily be high enough to make $f_{d} \approx 1$. With that in mind (2.131) can be written

$$
1-f_{i}-\frac{Z \rho f_{i}^{2}}{m_{i}} \frac{h^{3}}{\left(2 \pi m_{e} T_{e}\right)^{3 / 2}} \sum_{j \geqslant 1} j^{2} e^{E,\left(\rho, f_{i}\right) / T}=0 .
$$

As this equation is nonlinear, it must be solved by a Newton-type solver. 
As stated earlier, the Saha-Boltzmann approximation is inadequate to describe real plasma conditions. While agreeing on this point, Drawin and Emard $^{32}$ argue for a simplification to the entire collisional-radiative model (or rate equations) to a quasi-steady-state approximation which reduces the problem to an algebraic one. However, as they also note, this assumption is probably not valid for strong temporal variations in $n_{e}$ or $T_{e}$. Unfortunately, these are precisely the sorts of variations which occur in shock-dominated plasmas, and pinch experiments involve plasmas of that variety. It appears essential, therefore, to solve the entire set of rate equations for the ionization and radiation processes in order to accurately model these effects. Although it is beyond the scope of this thesis. impurity radiation effects will require a like treatment.

It was promised earlier that a means for finding $f_{i}^{m i n}$ would be given. To do this, observe that the initial conditions in an experiment should be uniform. That is, before any preionization mechanism is turned on, such as an $\mathrm{r}-\mathrm{f}$ field, the gas will be in an equilibrium state. Thus, $T_{e}=T_{i} \approx 300^{\circ} \mathrm{K}$ is a very good assumption, and the density can be readily estimated from the volume of the vacuum vessel and the amount of gas injected into the chamber. With $T_{e}$ and $\rho$ known, (2.126) and (2.131) can be solved simultaneously for $f_{i}$ and $f_{d}$. The value obtained for $f_{i}$ will be the appropriate $f_{i}^{m i n}$. 
It is of interest to note that great effort has be.. expended previously to find $f_{i}^{m i n}$ by an experimental diagnostic technique for use in a computer code. ${ }^{75}$ In that work, it was also observed that $f_{1}^{m i n}$ varied with the fill pressure, which is in agreement with the above arguments if we observe that varying the amount of gas injected into the apparatus will cause the density to vary and therefore the fill pressure as well.

\subsection{Boundary Conditions}

With the specification of the model equations for the interior region of the vacuum vessel, it remains $t$, develop the boundary conditions. This subject is more complicated than is generally understood because of the complex manner in which the plasma interacts with the wall. One immediate consequence of this interaction is that there can be significant charge separation in a boundary layer at the wall, invalidating all of the model equations except for the continuity equation. While this effect has heen studied, it has never been incorporated into a code where the boundary layer is matched to the interior via a continuity-type boundary condition, both regions having their own set of model equations. As will be clear from the discussion in the next chapter, this kind of approach would modify the solution technique proposed there in a complex manner. Hence, we also ignore the presence of a boundary layer. 
Another complex. set of boundary phenomena involve the breakdown of the wall from plasma interaction. Processes such as cracking, sputtering, evaporation, and blistering are among the processes that are very difficult to model but are important in a reactor environment. ${ }^{108}$ This is particularly so because of the impurities which find their way from the wall to the plasma where they can radiate away substantial amounts of the plasma's energy. Unfortunately, further study of these effects is outside the scope of this thesis.

Proceeding now with a set of boundary conditions which are a good starting point, we begin by allowing slip conditions at the wall. This is consistent with the neglect of the viscous terms in our MHD model and implies that the plasma flow is not frictionally impeded by the wall. Symbolically,

$$
\frac{\partial \boldsymbol{v}_{\|}}{\partial n}=0
$$

Next, we make the assumption of an adiabatic wall; i.e..

$$
\frac{\partial T}{\partial n}=0 .
$$

That this is a poor model of the temperature behavior is pointed out by $\mathrm{Chu}^{20}$ and Jensen. ${ }^{54}$ Regardless, restrictions in the computer algebra system being used forced the acceptance of the insulating wall condition. 
As the wall is non-conducting, it is assumed that

$$
\tilde{\boldsymbol{n}} \cdot \mathbf{B} \neq 0
$$

This amounts to saying that the full set ot equations in Faraday's law must be solved at the boundary. With this more accurate treatment, the special boundary condition in ANIMAL ${ }^{71}$ can be improved. Basically, that treatment allowed for the plasma to separate from the wall and return again. It was limited by the fact that the wall had to be a flux surface, i.e.. a conductor. Here, the collocation solution technique introduced in the next chapter implies no such restriction. The basic condition for separation is that the magnetic fjeld pressure exceed the sum of the partial pressures of the plasma species. That is, $v>0$ will occur if

$$
\frac{B^{2}}{8 \pi}>\sum_{a} p_{a}
$$

The precise value of $v_{2}$ is found by solving the momentum equation for $v_{2}$, and that equation takes full account of the different components of $B$ at the boundary.

While the plasma is not in contact with the wall, fictitious background gas is allowed to $\mathrm{flow}$ into and out of the region vacated by the plasma. This is a standard ploy to avoid numerical instabilities arising from the density 
dropping to a very low level. ${ }^{71}$ An alternate bit far more complex method would consist of following the vacuum-plasma interface and not solving any equations in the vacuum region.

Finally, the possible return of the plasma must be detected. This is accomplished by checking for the density rising above some arbitrarily predetermined cutof value. If it does $r i s e$ above that value, then $v$, is set to zero

Corner regions in the plasma are a little trickier to handle. Basically, it is necessary to check for $v$ being directed toward the interior of the domain before allowing the plasma to separate from the corner. Background gas flow and plasma return to the corner are handled as above. In the case of plasma returning to the wall, however, two components of must be set to zero instead of just $v$. This is due to the ambiguity of the symbol $v$, in a corner region.

One problem with this plasma-wall separation involves the return to and possible subsequent re-separation from the wall of the plasma. This "bouncing" process will impart some momentum to the wall and hence to the apparatus in any real experiment. In most cases, experiments are engineered so that very little movement in the parts can occur. Thus, any recoil in the apparatus due to a plasma bounce will be very rapidly damped away in a sinusoidal fashion. Because of this motion, some of the momentum absorbed by the apparatus could be reimparted to the plasma. One way to model this kind of behavior would be to 
treat the wall as being attached to a series of stiff springs instead of assuming it is rigid. Besause this logically implies a movement in other parts of the apparatus, in particular, in the coil system dealt with in Section 2.4 , this idea was not pursued any further.

\subsection{Initial Conditions}

Many simulation studies have assumed that a study of the preionization phase of a magnetically confined plasma could be bypassed because the plasma is essentially uniform after that phase is complete. Studies by Lindemuth et al.$^{75}$ have shown that this is far from what actually occurs. As the preionization phase is of critical importance to the amount of flux trapped by the plasma in Topolotron experiments, there has never been the temptation in this work to assume a uniform plasma state after preionization. Therefore, the initial conditions appropriate to our studies would be $T_{i}=T_{e} \approx 300^{\circ} \mathrm{K} ; \rho=\rho_{0}$, a uniform density in the vacuum vessel: $r=0$, meaning that there is no bulk motion in the gas: $\theta^{P}=0$, signifying that there are no currents in the gas; and finally, $f_{i}^{m i n}$ is determined from $T_{0}$ and $\rho_{0}$ as described earlier. 


\subsection{Driving Coil Model}

\section{$2.4 .1 \theta$-Coil}

While far less forbidding than the MHD problem, models of the driving coils are nonetheless difficult to solve in their own right. We begin with the $\theta$-coil which is by far the easier problem. The theory is then developed for the $I$ and $C$ coils, with generalizations sketched for arbitrary sets of toroidal conduc tors.

We oegin with Faraday's law:

$$
\mathcal{E}=-\frac{d}{d t} \Phi_{\varphi}=-\frac{d}{d t} \int B_{\varphi} d a
$$

Noting that the -field is composed of an external component (due to the $\theta$-coil) and a plasma component, we write

$$
\mathscr{E}=-\frac{d}{d t}\left(\int B_{\varphi}^{e x t} d a+\int B_{\varphi}^{p} d a\right)
$$

Since the field due to the $\theta-\operatorname{coil}$ is $\left(2 I^{\theta}\right) /(\mathrm{c} \rho)$, this becomes

$$
S=-\frac{2\left(z_{t o p}-z_{b o t t_{0 m}}\right)}{C} \log \left(\frac{\rho_{o u t}}{\rho_{i n}}\right) \frac{d I^{\theta}}{d t}-\frac{d}{d t} \int B_{\varphi}^{p} d a .
$$


Now the applied emf can be written

$$
\mathscr{E}=-V^{\theta}+R^{\theta} I^{\theta}+L_{\mathrm{e} f f} \frac{d I^{\theta}}{d t} \text {, }
$$

where

$$
L_{\text {eff }}=L_{\text {Vacuum }}^{\text {pactead }}+L_{\text {to }}
$$

Therefore, the state of this coil before crowbar is described by the lullowing set of equations:

$$
V^{\theta}-R^{\theta} I^{\theta}-\left[L_{e f f}+\frac{2\left(z_{t o p}-z_{b o t t o m}\right)}{c} \log \left(\frac{\rho_{o u t}}{\rho_{2 n}}\right)\right] \frac{d I^{\theta}}{d t}-\frac{d}{d t} \int b_{\dot{\psi}}^{v} d a=0
$$

and

$$
\frac{d V^{\theta}}{d t}=\frac{I^{\theta}}{C^{\theta}}
$$

After the circuit has been crowbarred, we simply ignore (2.143) and set $v^{\theta}$ to zero. This whole development is standard, except that the change in flux term involving $B_{\varphi}^{p}$ is over the plasma-free region inside the $\theta$-coil as well. Computing this contribution is accomplished by integrating the $\varphi$-component of the plasma field over the plasma-free region, so a means of computing the plasma 
field outside the plasma is required. Begin with the expression for the fleld due to a current distribution:

$$
\theta^{p \cdot i n s}(x)=\frac{1}{c} \nabla \times \int \frac{p\left(x^{\prime}\right) d^{3} x}{\left|x-x^{\prime}\right|}
$$

In our case, the integral is over the plasma region and must be done in rectangular components. ${ }^{52}$ Because of this, we give the rectangular components of $\boldsymbol{J}^{\mathbf{P}}:$

$$
\boldsymbol{P}=\left(\begin{array}{c}
J_{\rho}^{p} \cos \varphi-J_{\varphi}^{p} \sin \varphi \\
J_{\rho}^{p} \sin \varphi+J_{\varphi}^{p} \cos \varphi \\
J_{z}^{p}
\end{array}\right)
$$

Now cylindrical symmetry allows the observation point to be chosen in the $x-z$ plane $(\varphi=0)$ for purposes of evaluation. Since the integral is symmetric about $\varphi^{\circ}=0$ in the $\varphi^{\prime}$ integration, the $J_{\varphi}^{p} \cdot \sin \varphi^{\cdot}$ component of the $\hat{x}$ contribution to $J$ and the $J_{p}^{p} \cdot \sin \varphi^{\cdot}$ component of the $\hat{y}$ contribution to $\boldsymbol{J}$ integrate to zero. This leaves:

$$
\nabla^{p, i n s}(x)=\frac{1}{c} \nabla \times \int \frac{J_{\rho}^{p} \cdot \cos \varphi^{\prime} \hat{x}+J_{\varphi}^{p} \cdot \cos \varphi^{\prime} \hat{y}+J_{z}^{p} \cdot \hat{z}}{\sqrt{\left[\rho^{2}+\rho^{\prime 2}-2 \rho \rho^{\prime} \cos \varphi^{\prime}+\left(z-z^{\prime}\right)^{2}\right]}} \rho^{\prime} d \rho^{\prime} d \varphi^{\prime} d z^{\prime}
$$

Using integral 291.00 in Byrd and Friedman ${ }^{15}$ one obtains 


$$
\int_{0}^{2 \pi} \frac{d \varphi^{\prime}}{\sqrt{\left(a-b \cos \varphi^{\prime}\right)}}=2 \int_{0}^{\pi} \frac{d \varphi^{\prime}}{\sqrt{\left(a-b \cos \varphi^{\prime}\right)}}=\frac{4}{\sqrt{(a+b)}} K(k),
$$

where $K$ is the complete elliptic integral of the first kind. Choose $a$ and $b$ to be given by

$$
a=\rho^{2}+\rho^{\cdot 2}+\left(z-z^{\prime}\right)^{2}
$$

and

$$
b=2 \rho \rho^{\circ},
$$

so that $k$ is given by

$$
k=\frac{2 V\left(\rho \rho^{\prime}\right)}{\sqrt{\left[\left(\rho+\rho^{\circ}\right)^{2}+\left(z-z^{\prime}\right)^{2}\right]}} .
$$

One required integral thus becomes

$$
\int_{0}^{2 \pi} \frac{d \varphi^{\prime}}{\sqrt{\left[\rho^{2}+\rho^{\prime 2}-2 \rho \rho^{\prime} \cos \varphi^{\prime}+\left(z-z^{\prime}\right)^{2}\right]}}=\frac{4 K(k)}{\left.\sqrt{\left[\left(\rho+\rho^{\prime}\right)^{2}\right.}+\left(z-z^{\prime}\right)^{2}\right]}
$$

The two remaining integrals over $\varphi^{\prime}$ are identical and are found from equation (5.37) in Jackson 52 to be 


$$
\int_{0}^{2 \pi} \frac{\cos \varphi^{\circ} d \varphi^{\cdot}}{\sqrt{\left[{f^{2}}^{2}+\rho^{.2}-2 \rho \rho^{\circ} \cos \varphi^{\circ}+\left(z-z^{\circ}\right)^{2}\right]}}=\frac{2}{k \sqrt{ }\left(\rho \rho^{\circ}\right)^{\circ}}\left[\left(2-k^{2}\right) K(k)-2 E(k)\right] .
$$

When $\varphi=0$, observe that

$$
\hat{\rho}=\left.(\cos \varphi \hat{x}+\sin \varphi \hat{y})\right|_{\varphi=0}=\hat{x} .
$$

and

$$
\hat{\varphi}=\left.(\cos \varphi \hat{y}-\sin \varphi \hat{x})\right|_{\varphi=0}=\hat{y} .
$$

Hence, the equation for $\boldsymbol{\theta}^{p \cdot i n s}$ becomes

$$
\begin{aligned}
\boldsymbol{\theta}^{p \cdot i n s}(x)=\frac{2}{c} \nabla \times\{ & \hat{\rho} \int \frac{\left(2-k^{2}\right) K(k)-2 E(k)}{k \sqrt{ }\left(\rho \rho^{\prime}\right)} J_{\rho}^{p} \cdot\left(\rho^{\prime}, z^{\prime}\right) \rho^{\prime} d \rho^{\prime} d z^{\prime} \\
& +\hat{\varphi} \int \frac{\left(2-k^{2}\right) K(k)-2 E(k)}{k \sqrt{ }\left(\rho \rho^{\prime}\right)} J_{\varphi}^{p} \cdot\left(\rho^{\prime}, z^{\prime}\right) \rho^{\prime} d \rho^{\prime} d z^{\prime} \\
& \left.+\hat{z} \int \frac{2 K(k)}{\sqrt{\left[\left(\rho+\rho^{\prime}\right)^{2}+\left(z-z^{\prime}\right)^{2}\right]^{\prime}}} J_{z}^{p} \cdot\left(\rho^{\prime}, z^{\prime}\right) \rho^{\prime} d \rho^{\prime} d z^{\prime}\right\} .
\end{aligned}
$$

In order to express this vector in terms of known quantities, the plasma current density is expressed in terms of the plasma magnetic field. The component:s of the current density are

$$
J_{\rho}^{p} \cdot\left(\rho^{\prime}, z^{\prime}\right)=-\frac{c}{4 \pi} \frac{\partial B_{\varphi}^{p}}{\partial z^{\cdot}}
$$




$$
J_{\varphi}^{p} \cdot\left(\rho^{\cdot}, z\right)=\frac{c}{4 \pi}\left[\frac{\partial B_{\rho}^{p}}{\partial z}-\frac{\partial B_{z}^{p}}{\partial \rho}\right]
$$

and

$$
J_{z}^{p} \cdot\left(\rho^{\cdot}, z^{\prime}\right)=\frac{c}{4 \pi}\left[\frac{B_{\varphi}^{p}}{\rho}+\frac{\partial B_{\varphi}^{p}}{\partial \rho}\right]
$$

As only the $f$-component of the plasma fieat contributes to flux changes felt by the $\theta$-coil, only the $B_{\psi}^{\text {p.ins }}$ equation is given here:

$$
\begin{aligned}
& B_{\bullet}^{p, 2 \pi s}(\boldsymbol{x})=-\frac{1}{4 \pi} \int\left\{\frac{\partial B_{\varphi}^{p}}{\partial z} \frac{\partial}{\partial z}\left(\frac{2\left[\left(2-k^{2}\right) K(k)-2 \boldsymbol{E}(k)\right]}{k \vee(\rho \rho)}\right)\right. \\
& \left.+\left(\frac{B_{\varphi}^{p}}{\rho}+\frac{\partial B_{\varphi}^{p}}{\partial \rho}\right) \frac{\partial}{\partial \rho}\left(\frac{4 K(k)}{\sqrt{\left[(\rho+\rho)^{2}+\left(z-z^{\prime}\right)^{2}\right]}}\right)\right\} \rho d \rho^{\prime} d z .
\end{aligned}
$$

\subsubsection{Toroidal Coils}

With the $\theta$-coil calculation in hand, it remains to examine the toroidal conductors, the $l$ and $C$ coils. Because these coils are axisymmetric. they can be viewed as an infinite collection of circular current loops whose individual radil are infinitesimal. The interaction between any two of these loops is described by the mutual inductance law 92 


$$
\frac{d \Phi_{i j}}{d t}=\frac{d S_{i j}}{d I_{j}} \frac{d I_{j}}{d t}=M_{i j} \frac{d I_{j}}{d t} .
$$

This statement is valid in the limil of the two loops being rigid and the material making up the conductors having strictly linear magnetic characteristics. However, the conductors can be of any shape. with the geometric factor or mutual inductance, $M_{i, j}$, reflecting that information. Here, the coils are concentric circles of radii $\rho_{i}$ and $\rho_{j}$, with potentially different $z$ coordinates $z_{i}$ and $z_{j}$, and $M_{i, j}$ becomes

$$
M_{i j}=\frac{4 \pi V\left(\rho_{i} \rho_{j}\right)}{c^{2} k}\left[\left(2-k^{2}\right) K(k)-2 E(k)\right] .
$$

In this case, the modulus $k$ is given by

$$
k^{2}=\frac{4 \rho_{i} \rho_{j}}{\left(\rho_{i}+\rho_{j}\right)^{2}+\left(z_{i}-z_{j}\right)^{2}}
$$

To return to the infinite-dimensional case of the continuous conductor, the cross-section of the volume current is divided into very small squares, each of area $\Delta a$ :

$$
\mathscr{E}_{i}=-\sum_{j} \frac{d \Phi_{i j}}{d t}=-\sum_{j} M_{i j} \frac{d\left(\frac{I_{j}}{\Delta a}\right)}{d t} \Delta a
$$


Takıng the limit of zero area yields

$$
\mathscr{E}_{i} \underset{\Delta a \rightarrow 0}{\longrightarrow}-\int_{\text {surface }} M\left(\boldsymbol{r}_{i}, \boldsymbol{r}\right) \frac{\partial \boldsymbol{J}(\boldsymbol{r}, t)}{\partial t} \cdot \hat{\boldsymbol{n}} d a
$$

The simplest practical example of the use of this infinite-dimensional mutual inductance formulation is the circuit equation for the C-coil alone driving the plasma. It is given by

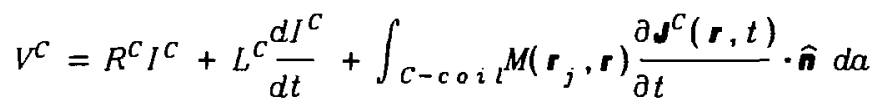

$$
\begin{aligned}
& +\int_{p \operatorname{lasma}} M\left(\boldsymbol{r}_{j}, \boldsymbol{r}\right) \frac{\partial \boldsymbol{P}^{P}(\mathbf{r}, t)}{\partial t} \cdot \hat{\boldsymbol{n}} d a
\end{aligned}
$$

where $r$, is any point on the $C$-coil, $R^{c}$ is the resistance of the circuit driving the $C$-coil, $L^{\mathrm{C}}$ is the inductance of that circuit, and $I^{\mathrm{C}}$ is the total current flowing in the $C$-coil:

$$
I^{C}=\int_{C-\operatorname{coi} l} \boldsymbol{J}^{C}(\boldsymbol{r}, t) \cdot \hat{\boldsymbol{n}} d a .
$$

While the circuit is not crowbarred, the time rate of change of the voltage can be written

$$
\frac{d V^{C}}{d t}=\frac{I^{c}}{C^{C}}
$$


where $C^{C}$ is the capacitance of the bank driving the $C$-coil. This development can now be used to write down the equations describing the interaction between the $I$ and $C$ coils and the plasma. In the simple case where separate capacitor banks drive the coils, the circuit equations for the two coils can be written

$$
\frac{d V^{I}}{d t}=\frac{I^{\prime}}{C^{\prime}}
$$

$$
\frac{d V^{C}}{d t}=\frac{I^{c}}{C^{C}},
$$

$$
\begin{aligned}
& V^{\prime}=R^{I} I^{\prime}+L^{\prime} \frac{d I^{\prime}}{d t}+\int_{l-\cos t} M\left(\boldsymbol{r}_{\imath}, \boldsymbol{r}\right) \frac{\partial d^{\prime}(\boldsymbol{r}, t)}{\partial t} \cdot \hat{\boldsymbol{n}} d a
\end{aligned}
$$

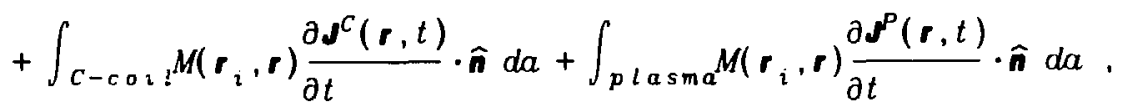

and 


$$
\begin{aligned}
& V^{C}=R^{C} I^{C}+L^{C} \frac{d I^{C}}{d t}+\int_{C-\operatorname{coi} l} M\left(\boldsymbol{r}_{j}, \boldsymbol{r}\right) \frac{\partial d^{C}(\boldsymbol{r}, t)}{\partial t} \cdot \hat{n} d a \\
& +\int_{l-\cos l} M\left(\boldsymbol{r}_{j}, \boldsymbol{r}\right) \frac{\partial \mathbf{d}^{l}(\boldsymbol{r}, t)}{\partial t} \cdot \hat{\boldsymbol{n}} d a+\int_{p l a s m a} M\left(\boldsymbol{r}_{j}, \boldsymbol{r}\right) \frac{\partial \boldsymbol{J}^{P}(\boldsymbol{r}, t)}{\partial t} \cdot \hat{\boldsymbol{n}} d a,
\end{aligned}
$$

where the 1 -coil variables are defined analogously to the $C$-coil variables, and $r_{2}$ is any point on the $I$-coil. When the coils are driven by a common capacitor bank, there is an additional constraint

$$
I^{T}=I^{\prime}+I^{C}
$$

To generalize this to $n$ coils, we can write

$$
\frac{d V^{a}}{d t}=\frac{I^{a}}{c^{a}},
$$

and

$$
\begin{aligned}
V^{a}=R^{a} I^{a}+L^{a} \frac{d I^{a}}{d t} & +\int_{a-\operatorname{coi} l} M\left(\boldsymbol{r}_{j}, \boldsymbol{r}\right) \frac{\partial\lrcorner^{a}(\boldsymbol{r}, t)}{\partial t} \cdot \hat{\boldsymbol{n}} d a \\
& +\int_{\text {others }} M\left(\boldsymbol{r}_{j}, \boldsymbol{r}\right) \frac{\partial \jmath^{\text {thers }}(\boldsymbol{r}, t)}{\partial t} \cdot \hat{\boldsymbol{n}} d a,
\end{aligned}
$$

where "others" includes the plasma as a conductor. A set of at most $n / 2$ constraints coupling subsets of these coils could be added to this description ( if $n$ is odd, then $(n-1) / 2$ is the maximum number of constraints). 
Once the currents on the coils are completely known, we can perform an integral over this current distribution to find the external magnetic field and its spatial derivatives in the plasma region. We can again use the basic formula

$$
\boldsymbol{B}(\boldsymbol{x})=\frac{1}{c} \nabla \times \int \frac{J\left(x^{\prime}\right) d^{3} x^{\prime}}{\left|x-x^{\prime}\right|},
$$

except now $x$ is in the plasma region and $J$ represents the coil currents, not the plasma currents. As previously indicated.

$$
B_{\varphi}^{e x t}=\frac{2 I^{\theta}}{c \rho}
$$

and this is due to the $\theta$-coil which has the only $\rho$ and $z$-directed currents contributing to $J$; the toroidal coils give rise to the other $B$-field components. To $f$ ind them, the integral

$$
\int \frac{J\left(x^{\prime}\right) d^{3} x}{\left|x-x^{\prime}\right|}
$$

must be evaluated. Since the toroidal coil current distribution can be written 


$$
J=J_{\varphi}\left(\rho^{\prime} . z^{\prime}\right) \hat{\varphi}=J_{\varphi}\left(\rho^{\prime}, z^{\prime}\right)[\cos (\varphi) \hat{y}-\sin (\varphi) \hat{x}] .
$$

the discussion in the last section concerning the $\varphi^{\prime}$ integration in (2.144) also applies to the $f^{\circ}$ integration here. Using (2.152), this leads to

$$
B_{\rho}^{\epsilon I t}(\rho, z)=-\frac{2}{c} \frac{\partial}{\partial z} \int \frac{J_{\varphi}\left(\rho^{\prime}, z^{\prime}\right)}{k v^{\prime}\left(\rho \rho^{\prime}\right)}\left[\left(2-k^{2}\right) K(k)-2 \boldsymbol{E}(k)\right] \rho^{\prime} d \rho^{\prime} d z^{\prime}
$$

and

$$
B_{z}^{e x t}(\rho, z)=\frac{2}{c \rho} \frac{\partial}{\partial \rho}\left\{\rho \int \frac{J_{\varphi}\left(\rho^{\prime}, z^{\prime}\right)}{k \sqrt{ }\left(\rho \rho^{\prime}\right)}\left[\left(2-k^{2}\right) K(k)-2 E(k)\right] \rho^{\prime} d \rho^{\prime} d z^{\prime}\right\},
$$

where the definitions of the last section apply. Using 710.00 and 710.02 of Byrd and Friedman, ${ }^{15}$ MACSYMA $^{81}$ can be used to perform the differentiation. This gives

$$
B_{\rho}^{\epsilon+{ }^{t}}(\rho, z)=-\frac{1}{2 c \rho^{3 / 2}} \int \frac{k\left(z-z^{\prime}\right) J_{\varphi}\left(\rho^{\prime}, z^{\prime}\right)\left[2\left(1-k^{2}\right) K(k)-\left(2-k^{2}\right) E(k)\right]}{\left(1-k^{2}\right) \sqrt{ } \rho^{\prime}} d \rho^{\prime} d z^{\prime} .
$$

and

$$
B_{z}^{e x t}(\rho, z)=\frac{1}{2 c \rho^{3 / 2}} \int \frac{k J \varphi\left(\rho^{\prime}, z^{\prime}\right)\left[2 \rho\left(1-k^{2}\right) K(k)+\left\{\rho^{\prime} k^{2}-\rho\left(2-k^{2}\right)\right\} E(k)\right]}{\left(1-k^{2}\right) \vee \rho^{\circ}} d \rho^{\prime} d z^{\prime} .
$$

Because the required spatial derivatives of these external field components are 
more complex, they were evaluated by MACSYMA and are not given here.

'Ihere are two special forms of $J_{\varphi}$ for the $l$ and $C$ coils in the approximation thist they have zero width. The first is for a horizontally-oriented conductor:

$$
J_{\varphi}\left(\rho^{\cdot}, z^{\prime}\right)=I\left(\rho^{\circ}\right) \delta\left(z^{\circ}-z_{0}\right)
$$

and the other for a vertically-oriented conductor:

$$
J_{\varphi}\left(\rho^{\prime}, z^{\prime}\right)=I\left(z^{\prime}\right) \delta\left(\rho^{\circ}-\rho_{o}\right)
$$

Thus, for the 1-coil, (2.181) becomes

$$
B_{\rho}^{e x t}(\rho, z)=-\frac{1}{2 c \rho^{3 / 2} \sqrt{ } \rho_{0}} \int \frac{k\left(z-z^{\prime}\right) I\left(z^{\prime}\right)\left[2\left(1-k^{2}\right) K(k)-\left(2-k^{2}\right) E(k)\right]}{1-k^{2}} d z^{\prime}
$$

while $(2.182)$ reduces to

$$
B_{z}^{e x t}(\rho, z)=\frac{1}{2 c, 0^{3 / 2} \vee \rho_{o}} \int \frac{k I\left(z^{\prime}\right)\left[2 \rho\left(1-k^{2}\right) K(k)+\left\{\rho_{0} k^{2}-\rho\left(2-k^{2}\right)\right\} E(k)\right]}{1-k^{2}} d z
$$

where 


$$
k^{2}=\frac{4 \rho \rho_{0}}{\left(\rho+\rho_{0}\right)^{2}+(z-z)^{2}} .
$$

Analogous expressions can be found for the conductors making up the $C$-coil. The total external $B$-field at a point, $x$, in the plasma region can then be found by adding the contributions of all of these conductors. 


\section{CHAPTER 3}

\section{SPATIAL DISCRETIZATION METHODS}

\subsection{General Spline Methods}

The spatial discretization methods which a:e going to be used here are the "collocation" methods." Basically, they are characterized by requiring that the differential equations or integral equations be satisfied exactly at a finite number of points in the spatial domain of the equations called the collocation points. The dependent variables and their spatial derivatives are then represented by piecewise polynomials chosen specifically for the problem at hand. For example, when treating the driving coils as one-dimensional. it is most advantageous to expand the current density in a set of B-splines (these are formally equivalent to piecewise polynomials) because the condition number of the matrix generated by applying the collocation method to the integral equations for the current density is smallest when B-splines are used as the basis functions. On the other hand, B-splines have their drawbacks in solving 
the MHD equations because the bandwidth of the matrix representing the problem expanded in B-splines is large.

One class of multidimensional splines is called tensor-product polynomial splines. If the tensor-product splines are chosen to be B-splines, they inherit the same bandwidth difficulty from the one-dimensional B-splines. Another set of multidimensional splines is called the piecewise polynomials of a given total order. ${ }^{104}$ Each polynomial involved contains products of powers of the independent variables where the sum of the powers involved in any given term does not exceed the given total order. Before specializing to the spline developments of Rubin and Khosla, ${ }^{99}$ it is best to review some basic spline theory. First, define a partition of the d-dimensional domain of interest as follows:

$$
\Delta_{2}: a_{2}=x_{1,1}<x_{1,2}<. . .<x_{i, n,}=b_{i}, 1 \leqslant i \leqslant d .
$$

That is, (3.1) defines a partition for each spatial dimension in the problem in terms of $\left\{x_{2 k}\right\}$, a set of breakpoints. On each rectangular region (or interval for $d=1$ ) defined by this partition, we choose a set of polynomial basis functions in which to expand our functions. For polynomials of total order $p$, these basis functions are of the form

$$
z_{1}^{l} \cdot \cdot \cdot z_{d}^{l},
$$


with

$$
l_{1}+l_{2}+\ldots+l_{d} \leqslant p
$$

For tensor product polynomial splines, this condition is changed to

$$
l_{1}, l_{2}, \cdot, \cdot, l_{d} \leqslant p .
$$

Also needed to specify the spline function over the larger domain are the continuity conditions across breakpoints. Each smoothless constraint removes one degree of freedom from the spline and thus lowers the dimensionality of the problem to be solved.

To make these ideas concrete, we apply them to the approximation of a dependent variable represented by $u$ in the $\rho-z$ plane. On the $k, l \frac{t h}{s m a l l}$ rectangle defined by choosing a set of breakpoints, this variable can be expressed as

$$
\mathscr{H}(\rho, z)=\sum_{i=0}^{p} \sum_{j=0}^{p} a_{i, j}\left(\frac{\rho-\rho_{k}}{h_{k}}\right)^{i}\left(\frac{z-z_{l}}{k_{l}}\right)^{j}
$$

which is a two-dimensional tensor product spline, or as a two-dimensional polynomial spline of total order $p$ : 


$$
\mathscr{f}(\rho, z)=\sum_{i=0}^{p} \sum_{j=0}^{p-i} a_{i, j}\left(\frac{\rho-\rho_{k}}{h_{k}}\right)^{l}\left(\frac{z-z_{l}}{k_{l}}\right)^{j}
$$

since (3.6) is more efficient computationally, it would be advantageous to use splunes of total oider $p$. Unfortunately, the physical nature of our probleit dictales that $u$ should be continuous across breakpoints along with all first and second derivatives. That is, we assume there are no shock discontinultes. This leads to a number of constraints on the spline, more, for example, than are avallable to a two-dimensional polynomial spline of total order 3 or 5 . To remedy this problem, we could adi jusi enough higher-order polynomial terms to the "total order" polynomials so that there would be sufficient degrees of freedom. But this is rather ad hoc, so the tensor product splines, which do have enough degrees of freedom, will suffice for now. The situation is not as bad as it would seem, however, because multidimensional tensor-product B-splines have an equivalent piecewise polynomial representation. ${ }^{104}$ And using the "compact" specialized splines of Rubin and Khosla.102 one can obtain a block tridiagonal matrix representation of the problem, which is of greatly reducfod dimension. 


\subsection{A Two-Dimensional Spline}

The Spline 4 is the "compact" representation spline"101 we wish to use here, so a characterization of its two-dimensional extension (Spline 4 is a one-dimensional spline) will be given. First, the one dimensional Spline 4 is a $C^{2}$ quintic spline. Its first derivative coefficient, $m_{j}$, is chosen to satisfy certain cubic spline relationships 100

$$
\begin{aligned}
& m_{j}=\frac{h_{j}}{3} K_{j}+\frac{h_{j}}{6} K_{j-1}+\frac{u_{j}-u_{j-1}}{h_{j}} \\
& m_{j}=-\frac{h_{j+1}}{3} K_{j}-\frac{h_{j+1}}{6} K_{j+1}+\frac{u_{j+1}-u_{j}}{h_{j+1}},
\end{aligned}
$$

where $u_{j}$ is the functional value at the $j \underline{t h}$ grid point, $K_{j}$ is the cubic spline second derivative coefficient, and $h_{j}$ is grid spacing, i.e.. the distance between the $j-1 \underline{\mathrm{s}}$ and $j \underline{\mathrm{th}}$ breakpoints. The second derivative coefficient, $G_{j}$. is given by

$$
G_{j}=K_{j}+\frac{h_{j}^{3}+h_{j+1}^{3}}{6 h_{j+1}\left(h_{j}+h_{j+1}\right)^{2}}\left[K_{j+1}-\left(1+\frac{h_{j+1}}{h_{j}}\right) K_{j}+\frac{h_{j+1}}{h_{j}} K_{j-1}\right],
$$

where again, the $K_{j}$ 's are the cubic spline second derivative coefficients and satisfy the relation 


$$
\frac{h_{j}}{6} K_{j-1}+\frac{h_{j}+h_{j+1}}{3} K_{j}+\frac{h_{j+1}}{6} K_{j+1}=\frac{u_{j+1}-u_{j}}{h_{j+1}}-\frac{u_{j}-u_{j-1}}{h_{j}}
$$

In the collocation method, the functional value, $u_{j}$, is not given but is determined by forcing the equation (differential or integral) it satisfies to hold exactly at the $j \underline{\text { th }}$ breakpoint. While the collocation method does not require that the collocation points coincide with the breakpoints, the spline conditions on $m$, and $K_{j}$ at the breakpoints force one to collocate also at the breakpoints if first and second spatial derivatives occur in the defining equation for $u$. It is important to note that equations (3.7) allow the spline first derivative variable, $m_{j}$, to be eliminated in favor of the function of interest, $u$, and the spline second derivative, $K_{j}$. Note also that this reduction can occur at the boundary as well because of the two one-sided representations for $m_{j}$. The addition of a boundary condition for $K$, at each boundary, together with a means of specifying $u$ at every breakpoint (boundaries included), completely determines all spline coefficients.

two dimensions, the situation is much more complicated. To begin with, the number of spline coefficients to be determined has just been squared. Also, mixed derivatives appear wl: ch have not been dealt with so far. However, there are analogs of $m_{j}$ and $K_{j}$ in the second dimension. These will be denoted $l$, and $L_{j}$. 
A simple inventory reveals that there are four more coefficients per breakpoint which are required to specify the bi-quintic's 36 coefficients on each subdomain of the mesh. Given that continuity of the second derivative is assumed in each coordinate direction, it makes sense to assume that

$$
\frac{\partial^{2} u}{\partial x_{1} \partial x_{2}}, \frac{\partial^{3} u}{\partial x_{1}{ }^{2} \partial x_{2}}, \frac{\partial^{3} u}{\partial x_{1} \partial x_{2}{ }^{2}}, \text { cond } \frac{\partial^{4} u}{\partial x_{1}{ }^{2} \partial x_{2}{ }^{2}}
$$

are also continuous. In this case, Rubin and Khosla ${ }^{99}$ give spline relations for the mixed second partial, denoted here by $\hat{l}_{i, j}$ or alternatively by $\widehat{m}_{i, j}$ :

$$
\begin{aligned}
& \frac{1}{k_{j}} \hat{l}_{i, j-1}+2\left(\frac{1}{k_{j}}+\frac{1}{k_{j+1}}\right) \hat{l}_{i, j}+\frac{1}{k_{j+1}} \hat{l}_{i, j+1}= \\
& \frac{3}{k_{j+1}^{2}}\left(m_{i, j+1}-m_{i, j}\right)+\frac{3}{k_{j}^{2}}\left(m_{i, j}-m_{i, j-1}\right) \\
& \frac{1}{h_{i} \hat{m}_{i-1, j}}+2\left(\frac{1}{h_{i}}+\frac{1}{h_{i+1}}\right) \hat{m}_{i, j}+\frac{1}{h_{i+1}} \hat{m}_{i+1, j}= \\
& \frac{3}{h_{i+1}^{2}}\left(l_{i+1, j}-l_{i, j}\right)+\frac{3}{h_{i}^{2}}\left(l_{i, j}-l_{i-1, j}\right)
\end{aligned}
$$

However, Rubin and Khosla do not give spline relations for the third and fourth mixed derviatives which must be found in order to completeiy specify the two-dimensional interpolant. This interpolant is important, for example, in performing integrations where the integrand contains the function which has bcen spline fit. The interpolant would also be essential in adaptive mesh or moving 
grid applications. Consequently, the spline relations are developed in Appendix B. Using the notation

$$
\begin{aligned}
& \left.\frac{\partial^{3} u}{\partial x_{1}{ }^{2} \partial x_{2}}\right|_{i, j}=\hat{K}_{i, j}, \\
& \left.\frac{\partial^{3} u}{\partial x_{1} \partial x_{2}^{2}}\right|_{i, j}=\hat{L}_{i, j} .
\end{aligned}
$$

and

$$
\left.\frac{\partial^{4} u}{\partial x_{1}{ }^{2} \partial x_{2}{ }^{2}}\right|_{i, j}=\hat{T}_{i, j}
$$

these conditions can be written:

$$
\begin{aligned}
\frac{1}{k_{j}} \hat{K}_{i, j-1} & +2\left(\frac{1}{k_{j}}+\frac{1}{k_{j+1}}\right) \hat{K}_{i, j}+\frac{1}{k_{j+1}} \widehat{K}_{i, j+1}= \\
& \frac{3}{k_{j+1}^{2}}\left(G_{i, j+1}-G_{i, j}\right)+\frac{3}{k_{j}^{2}}\left(G_{i, j}-G_{i, j-1}\right) .
\end{aligned}
$$




$$
\begin{aligned}
\frac{1}{h_{i}} \hat{L}_{i-1, j} & +2\left(\frac{1}{h_{i}}+\frac{1}{h_{i+1}}\right) \hat{L}_{i, j}+\frac{1}{h_{i+1}} \hat{L}_{i+i, j}= \\
& \frac{3}{h_{i+1}^{2}}\left(H_{i+1, j}-H_{i, j}\right)+\frac{3}{h_{i}^{2}}\left(H_{i, j}-H_{i-1, j}\right),
\end{aligned}
$$

and

$$
\begin{aligned}
k_{j} T_{i, j-1}+ & 2\left(k_{j}+k_{j+1}\right) T_{i, j}+k_{j+1} T_{i, j+1}= \\
& \frac{6}{k_{j+1}}\left(G_{i, j+1}-G_{i, j}\right)-\frac{6}{k_{j}}\left(G_{i, j}-G_{i, j-1}\right)
\end{aligned}
$$

with

$$
\widehat{T}_{i, j}=T_{i, j}+\frac{k_{j}^{3}+k_{j+1}^{3}}{6 k_{j+1}\left(k_{j}+k_{j+1}\right)^{2}}\left[T_{i, j+1}-\left(1+\frac{k_{j+1}}{k_{j}}\right) T_{i, j}+\frac{k_{j+1}}{k_{j}} T_{i, j-1}\right] \text {, }
$$

where $k_{j}$ is the distance between the $i, j+1 \underline{s t}$ and $i, j \underline{t h}$ breakpoints, and $H_{i, j}$ is the spline second derivative with respect to the second coordinate variable.

In order to have a consistent approximation at the boundaries, it remains to find more accurate one-sided approximations to $\hat{\imath}, C, H, \hat{K}, \hat{L}$, and $\hat{T}$ at the boundaries. That is, spline boundary conditions are required in addition to the physical boundary conditions. Derivation of the added boundary conditions has also been done in Appendix B, and leads to three- and four-point boundary conditions. For the results, we refer to that appendix. These boundary conditions must be computed to the same accuracy as the solution in the 
interior. Otherwise, the spline boundary conditions will degrade the accuracy. of the interior solution with time. ${ }^{114}$

With all of the coefficients in the spline now determined, we could write down explicitly the bi-quintic interpolant. However, we do not yet know the coefficients of the interpolant explicitly because they are a non-trivial linear combination of the spline coefficients discussed above. The precise linear combinations are given in Appendix $C$, logether with a means of inverting that linear system so as to obtain the $a_{i, j}$ in equation (3.5), the interpolant's coefficients, in terms of spline variables and $u_{i, j}$.

\subsection{Discretization of the MHD Equations}

Turning now to the MHD equations, the spatial discretization is not yet totally determined because we have not specified how the first derivative replacements are to be performed. As noted earlier, there is no choice in this matter on the boundary: the one-sided form to the interior of the mesh is the one to use. For example, on the left boundary of the first coordinate, the following replacement is done:

$$
\left.\frac{\partial u}{\partial x_{1}}\right|_{1, j}=-\frac{h_{2}}{3} K_{1, j}-\frac{h_{2}}{6} K_{2, j}+\frac{u_{2, j}-u_{1, j}}{h_{2}} .
$$


In order to make a sensible first derivative replacement choice at interior mesh points, we turn now to the subject of "upwinding." It is a well established fact that in advection or convection dominated flows, there are serious numerical instabilities introduced by using a second-order approximation to first derivatives. ${ }^{6}$ These instabilities are generally damped away by using an artificial viscosity treatment, ${ }^{95}$ essentially modifying the original equation by adding an effective numerical diffusion to it. Also, the true approximation becomes first-order instead of the second-order one that we started with, and there is evidence that the damage may be worse than that. ${ }^{42}$ Since this technique has been in use for so long in computational physics circles, there has developed a sort of folklore associated with its use. While others ${ }^{28}$ have noted that higher-order approximation techniques lead to improved behavior. Leonard 66.68 exploded the artificial viscosity myth and provided some new techniques for stabilizing errors due to strong convection. He demonstrated that even-order approximations of first derivatives cause amplification of numerical errors arising from convection-dominated flow, while odd-order approximations tend to stabilize these errors. Parenthelically, it is curious that Khosla and Rubin, ${ }^{58}$ while recognizing that first-order upwinding was too severe in its suppression (filtering) of instabilities, chose a less severe "filter" approach by using a second-order parametrized modification to central differencing that is unstable. Of the polynomial methods Leonard examined, the third-order or cubic techniques appear to give the best results. In the spline considered here, fortuitiously enough, the one-sided first derivative 
approximations happen to be third-order; the exact truncation error is given in Appendix B. "Upwinding" can therefore be done implicitly and consistently in the bi-quintic spline context by choosing the appropriate one-sided form of $m_{j}$. This is based on local determinations of the "upwind" direction during the course of each solution iteration in the time integration of the MHD equations.

All first derivative terms in the model equations are subject to this oscillatory error, not just the derivatives of the density, $\rho .{ }^{72}$ Consequently, in more complex equations. a means of treating all first derviatives in a unified way is essential. This brings us face to face with another key issue in solving partial differential equations. The substance of this issue is whether conservation of energy and other physical quantities will be enforced on the computational mesh or not. Putting the PDEs into "conservation form"70 (also known as "flux form" or "divergence form") is the standard means of conserving one or more physical quantities described by the finite-dimensionalized equations. In contrast, "advective form"59 and a very similar structuring we call "convective form" do not conserve any physical quantities. And while there may be some superior form of the model equations, the present controversy in computational physics revolves around which of these two approaches is superior.

The conservation form of an equation for the dependent variable $u$ can be written 


$$
\frac{\partial u}{\partial t}+\sum_{i=1}^{d} \frac{\partial f_{i}(\boldsymbol{g})}{\partial x_{i}}=0
$$

where is the vector of dependent variables, $f_{i}$ is an arbitrary function of (possibly involving spatial differential operators), $d$ is the dimension of the

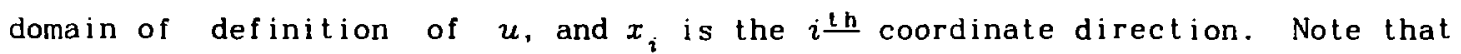
the MHD equations of Chapter 2 cannot be put into the form of $(3.20)$, or any reasonably relaxed definition of conservation form because of the presence of effective "source" terms. These are the radiation loss term in the energy equation, the heat transfer terms in both energy equations, and all mixed derivative and product derivative terms. As a result, numerical consistency is lost, rigorous energy and magnetic field conservation is not possible in our MHD model, and the benefit of using conservation form thus becomes suspect. In the context of the higher-order spline formulation, conservation form requires that each $f_{i}$ in $(3.20)$ be treated as a dependent variable for which yet another spline equation must be solved. ${ }^{100}$ This would lead to hundreds of variables to solve for at each collocation point on the mesh, and thereby renders impossible an already difficult problem. Another source of difficulty is the inability to enforce the constraint $\nabla \cdot \theta=0$ with these methods. ${ }^{8} \cdot 3$. This is not to say that conservation form is not a viable form of the equations to use, simply that it should be used when the model equations represent conservation laws, and with some skepticism otherwise. 
An equation is in convective form, on the other hand, if it can be described by

$$
\frac{\partial g_{i}}{\partial t}=\sum_{j=1}^{N} \sum_{k=1}^{d} r(\boldsymbol{g}) \frac{\partial g_{j}}{\partial x_{k}}+S_{i}(\boldsymbol{\sigma})
$$

where $g_{2}$ is the $i \underline{t h}$ dependent variable. $N$ is the number of dependent variables, $r(\boldsymbol{g})$ is a rational multivariate polynomial in the dependent variables, and $S_{i}(\boldsymbol{\theta})$ is an effective source term including second derivative terms, source and sink terms, and other terms not involving derivatives. This form of the equations makes no pretention of satisfying any conservation condition exactly. However, when higher-order splines are used with optimal placement of the mesh points, the integrated error in the conserved variables should be small. If, on the other hand, artificial viscosity treatments were applied to (3.21), very poor conservation results could be expected.

The most important feature of (3.21) is that it provides the natural framework required to deal uniformly with the "upwinding" issue. That is, the first derivative terms in $(3.21)$ can be discretized as

$$
\left.\left.\left[r(\boldsymbol{\sigma}) \frac{\partial g_{j}}{\partial x_{k}}\right]\right|_{\star} \longrightarrow r(\boldsymbol{\sigma})\right|_{*} \begin{cases}m_{*,+}^{k_{*}} & ,\left.r(\boldsymbol{\sigma})\right|_{*}>0 \\ m_{*}^{k_{*}-} & ,\left.r(\boldsymbol{\sigma})\right|_{*}<0\end{cases}
$$

where $x$ is a collocation point in the $d$-dimensional space, and $m_{x}^{k_{*}+}$ and $m_{*}^{k} \cdot-$ denote the one-sided approximations to $\partial_{x_{k}} g_{j}$. The only possible ambiguity in 
this formulation is the treatment of product derivative terms. As only one of the derivatives can be represented by the explicit derivative on the left-hand side of (3.22), the other derivative in the product must be included in $r(\boldsymbol{g})$. The selection of which of the derivatives to include is an arbitrary choice.

Another interesting property of (3.21) is that constraints such as $\nabla \cdot B=0$ can be explicitly included in the equations if that is desired. Including constraints would be accomplished by algebraic expansion of the right-hand side of (3.21), so that all possible cancellation occurs. A restructuring into the original general form would follow.

With these advantages, it would appear that convective form is the structure to use for complex MHD equations. And perhaps so. However, lest this discussion mislead some, it should be made clear that the stabilization of convection errors and use of higher-order splines does not constitute a panacea for all numerical instabilities. The temporal discretization instabilities discussed in Chapter 4 will make this clear. Moreover, in the spatial domain, these methods will not suppress the "wiggle" growth associated with not adequately resolvirig the structure of shocks. The AMM and AMR techniques discussed in Chapter 1 are required to cope with those difficulties; however. they will not be developed here for the bi-quintic spline framework.

The observation which led Leonard to the correct methods for upwinding was that a negative feedback stabilization mechanism must be available to damp out numerical errors. ${ }^{67}$ While the dominant spatial discretization errors have 
traditionally come from amplification of first derivative numerical modelline errors, Leonard's viewpoint also identified second derivative terms as a potential source of error growth. Second derivative terms are a "potential" source of error not because of the "centered" approximations normally used for the derivative, but because of the coefficient multiplying the second derivative. For stable numerical behavior, these coefficients must be posilive. and they normally are. When they are negalive numerically, it is a non-physical effect resulting from the undershooting of the spline approximation of a physically non-negative dependent variable, occurring in a shock-1ike or steep gradient region. The remedy for second derivative errors is to enforce positivity of all second derivative coefficients. This is done by replacing all negalive quantities contributing to the coefficient by an appropriate smal! positive "floor" value for that variable.

The spatial discretization of the MHD equations is accomplished by first putting those equations into convective form. Next, the substitutions implied by (3.22) are done. Then, the following second derivative transformations take place :

$$
c,\left.\frac{\partial^{2} u}{\partial x_{1}^{2}}\right|_{i, j} \longrightarrow \bar{c}, G_{i, j}
$$




$$
\left.c_{2} \frac{\partial^{2} u}{\partial x_{2}{ }^{2}}\right|_{i, j} \longrightarrow \bar{c}_{2} H_{i, j}
$$

and

$$
\left.c_{3} \frac{\partial^{2} u}{\partial x, \partial z_{2}}\right|_{i, j} \longrightarrow \bar{c}_{3} \hat{l}_{i, j}
$$

where the $c_{i} \rightarrow \vec{c}_{i}$ transformation is done to assure positivity. Tlere are still spatial derivalive operators left over, but they do not operate on the dependent variables. Typically, they involve the external driving magnetic field, and are assumed to be known explicitly from some other computation.

In the above discretization, it is assumed that the MHD equations do not involve third or higher-crder spatial derivatives. Nonetheless, even the elimination of the spline first derivatives using (3.7) leaves us with four variables to solve for $\left(u_{i, j}, K_{i, j}, L_{i, j}\right.$, and $\left.\hat{l}_{i, j}\right)$ per dependent variable per grid point. In the sequel, it is assumed that there are $N$ gridpoints in the $x_{1}$-direction and $M$ grid points in the $x_{2}$-direction, and that the MHD variables are $\rho, v_{1}, v_{2}, v_{3}, T_{e}, T_{i}, B_{1}^{p}, B_{2}^{p}$, and $B_{3}^{p}$. If we make the definitions 


$$
\begin{aligned}
& \boldsymbol{u}_{1}=\left[\rho_{1,1}, K_{1,1}^{o}, L_{1,1}^{o}, \hat{l}_{1,1}^{p},\left(v_{1}\right), 1,1, K_{1,1}^{v}, L_{1,1,}^{v}, \hat{l}_{1,1, \ldots}, \ldots\right. \\
& \left(B_{3}^{p}\right), \ldots, K_{1,}^{B_{3}^{p}}, L_{1,1}^{B_{3}^{p}}, \hat{l}_{1,1}^{B_{3}^{p}}, \\
& \rho_{2,1}, K_{z, 1}^{\rho}, L_{2,1}^{\rho}, \hat{l}_{2,1}^{\rho},\left(v_{1}\right)_{2,}, K_{2,1}^{v}, L_{2,1}^{v}, \hat{l}_{2,1}^{v}, \ldots,
\end{aligned}
$$

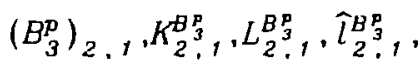

$$
\begin{aligned}
& \rho_{N, M}, K_{N, M}^{\rho}, L_{N, M}^{\rho}, \hat{l}_{N, M}^{\rho},(v,)_{N, M}, K_{N, M}^{v,}, L_{N, M}^{v}, \hat{l}_{N, M}^{v}, \ldots, \ldots \\
& \left.\left(B_{3}^{p}\right)_{N, M}, K_{N, M}^{B^{p}}, L_{N, M}^{B^{p}}, \hat{l}_{N, M}^{B^{p}},\right]^{T},
\end{aligned}
$$

and

$$
\begin{gathered}
\boldsymbol{u}_{2}=\left[\rho_{1}, 1,0,0,0,\left(v_{2}\right)_{1,1}, 0,0,0, \ldots,\left(B_{3}^{p}\right)_{1}, 0,0,0,0,\right. \\
\rho_{2,1}, 0,0,0,\left(v_{1}\right)_{2,1}, 0,0,0, \ldots,\left(B_{3}^{p}\right)_{2,}, 0,0,0 \\
. . \\
\left.\rho_{N, N}, 0,0,0,\left(v_{1}\right) \ldots, 0,0,0, \ldots,\left(B_{3}^{p}\right)_{N, N}, 0,0,0\right]^{T},
\end{gathered}
$$

then the spatially discretized MrD equations can be written

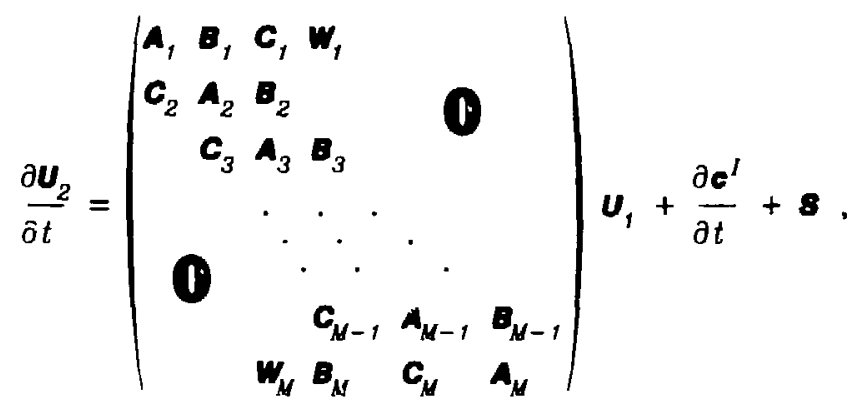

where $\boldsymbol{s}$ is a "source" vector containing all terms not dependent upon $\boldsymbol{U}_{2}$; the 
time derivative of $c^{\prime}$ indicates an explicit dependence on the external coil currents; and the matrices $\boldsymbol{w}_{\boldsymbol{f}}$ and $\boldsymbol{w}_{\boldsymbol{\mu}}$ are block diagonal. The $\boldsymbol{A}_{\boldsymbol{i}}$ 's have the form

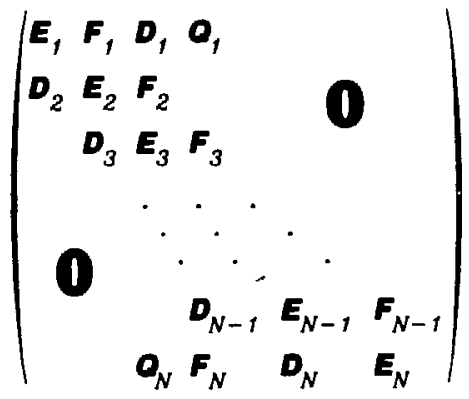

The $\boldsymbol{C}_{i}$ 's and $\boldsymbol{\theta}_{i}$ 's have $\mathrm{e}$ ither the lower-diagonal form

$$
\left(\begin{array}{cccc}
v_{1} & & & \\
p_{2} & v_{2} & & 0 \\
& p_{3} & v_{3} & \\
1 & \ddots & \cdot & \cdot \\
0 & & p_{N} & v_{N}
\end{array}\right)
$$

or the upper-diagonal transpose of it. The ambiguity here arises from the two one-sided equations (3.7) which can be used in the mixed derivative relations (3.11). So the overall matrix structure in (3.28) is essentially seven-banded with extended boundary treatment bands, and the basic matrix blocks are $36 \times 36$. 


\subsection{Spatial Discretization of the Coil Equations}

In accordance with the discussion in Chapter 2 on the computallon of $B_{\rho}^{x t}$ anci $B_{z}^{e x t}$, we assume that the toroidal coils are so thin that the rurrent distribution in them is one-dimensional. It can certainly be argued lial lhi current is all in a thin surface layer if the frequency in the driving circull is high enough. ${ }^{2}$ The current distribution in these coils ls therefore represented by one-dimensional splines. Because the matrix representat ion for this problem will turn out to be dense, the best numerically conditioned matix arising from the collocation method should be sought. B-splines provide thrt good numerical conditioning. On the $I$-coil for example.

$$
J^{\prime}(z)=I^{\prime}(z) \hat{\varphi} \approx \hat{\varphi} \sum_{i=1}^{N^{\prime}} c_{i}^{\prime} N_{i}^{m}(z)
$$

where $N_{l}^{m}(z)$ is the $i$ th normalized B-spline of order $m$ on the interval def lned by the I-coil. The details of how a given B-spline is evaluated on a given mesh with predetermined continuity conditions at the breakpoints will be left to Schumaker ${ }^{104}$ and de Boor ${ }^{7}$ who both deal with the subject very lucidly. However, there is still the question of what continuity conditions should be imposed and where the collocation points should be placed in view of those conditions. But first, the nature of the collocation problem should be made clear. 
For the I-coil alone. the integro-differential equation (2.174) describes the problem. Using the approximation (3.31), the spatially discretized version is

$$
\begin{aligned}
& V^{I}=R^{\prime} \int_{z,}^{z_{2}} d z \sum_{i=1}^{M^{\prime}} c_{i}^{I} N_{i}^{m}(z)+\int_{z,}^{z_{2}} d z\left[L^{I}+M\left(\rho^{I}, z_{j}, \rho^{I}, z\right)\right] \sum_{i=1}^{M^{\prime}} \frac{d c_{i}^{I}}{d t} N_{i}^{m}(z) \\
& +\int_{p \operatorname{lasma}} M\left(\rho^{I}, z_{j}, \boldsymbol{r}\right) \frac{\partial J_{\varphi}^{p}}{\partial t} d a \text {. }
\end{aligned}
$$

where $z_{j}$ is one of the collocation points on $\left[z_{1}, z_{2}\right]$. Now the last term, the integral over the plasma region couples into the calculation for the $c_{i}, B_{p}^{p}, B_{z}^{p}$ and all of their spline coefficients ( 9 each) at every mesh point in the plasma domain. Clearly, this makes the matrix representation for the problem enormous. Even acceptance of a less accurate two-dimensional quadrature by involving fewer plasma magnetic field variables would not decouple any of the plasma grid points, and therefore, sparse matrix solvers will be of no assistance. Hence. the plasma integral is assumed known explicitiy. Writing

$$
f^{p}\left(z_{j}\right) \equiv \int_{p l a s m a} M\left(\rho^{I}, z_{j}, r\right) \frac{\partial J_{\varphi}^{p}}{\partial t} d a .
$$

equation ( 3.32$)$ becomes: 


$$
\begin{gathered}
\sum_{i=1}^{N^{\prime}}\left\{L^{\prime} \int_{z_{1}}^{z_{2}} N_{i}^{m}(z) d z+\int_{z,}^{z_{2}} M\left(\rho^{\prime}, z_{j}, \rho^{\prime}, z\right) N_{i}^{m}(z) d z\right\} \frac{d c_{i}^{l}}{d t}= \\
V^{I}-R^{I} \sum_{i=1}^{N^{I}}\left[\int_{z,}^{z_{2}} N_{i}^{m}(z) d z\right] c_{i}^{\prime}-f^{p}\left(z_{j}\right) .
\end{gathered}
$$

This can be written in matrix notation as

$$
\left(L^{\prime} \mathbf{P}+\boldsymbol{U}\right) \frac{d \boldsymbol{c}^{\prime}}{d t}=\bullet-R^{l} \mathbf{P c}^{\prime}
$$

where

$$
(P)_{i j}=\int_{z,}^{z_{2}} N_{i}^{m}(z) d z
$$

$$
\text { (M) }{ }_{i j}=\int_{z,}^{z} M\left(\rho^{l}, z_{j}, \rho^{l}, z\right) N_{i}^{m}(z) d z \text {. }
$$

and

$$
(\bullet)_{i}=V^{I}-f^{p}\left(z_{i}\right)
$$

As the coupled $I$ and $C$-coil system has a very similar matrix representation, il 
will not be given here. To study the behavior of equation (3.35), the spatial

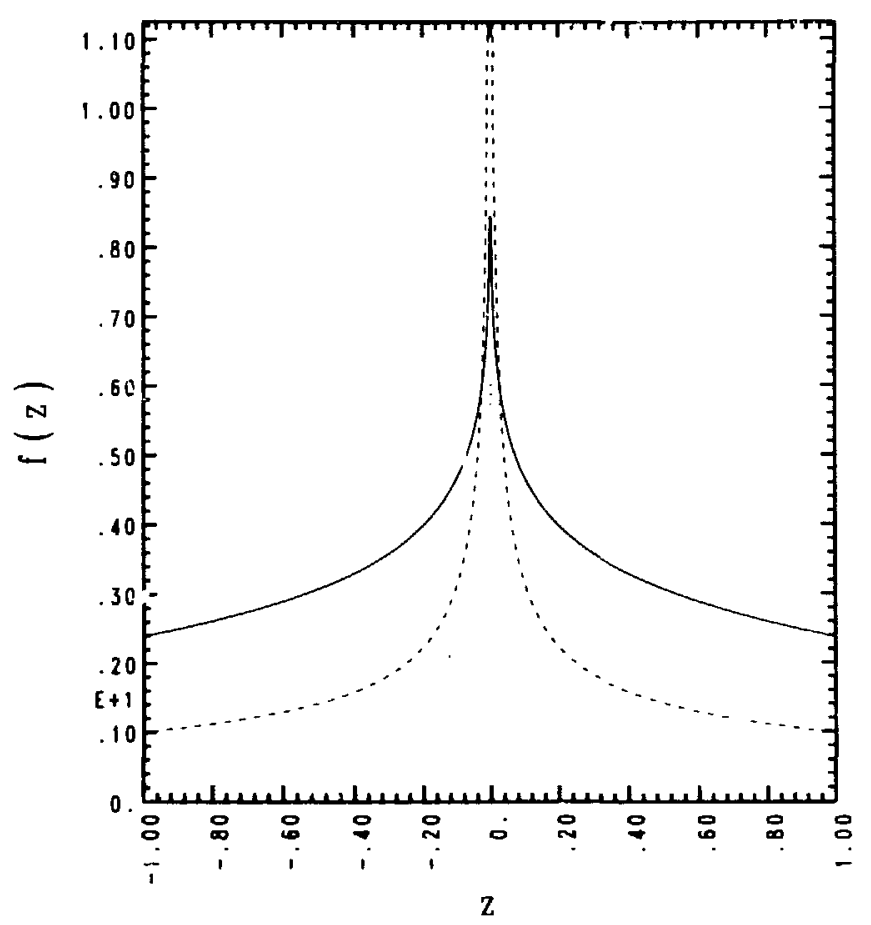

Figure 2.i. Three functions with singularities at $\mathrm{z}=0$ are depicted. $V(1 /|z|)$ is the non-integrable singularity (dashed line), while both $-\log (|z|)$ (dotted line) and $M\left(z^{\prime}, z\right)$ (solid line) are integrable and of logarithmic character.

discretization of a Fredholm integral equation of the first kind, the numerical character of a similar problem will be studied:

$$
\boldsymbol{M r}=\boldsymbol{\bullet}
$$

which is the spatial discretization of the integral equation 


$$
\int_{x_{1}}^{x_{2}} M\left(x^{\cdot}, x\right) f(x) d x=g(x), \quad x, \leqslant x \leqslant x_{2} .
$$

Typically, this integral equation represents an ill-posed problem; i.e., $\boldsymbol{M}$ has a very bad condition number. ${ }^{76}$ However, when $M\left(x^{\prime}, x\right)$ is non-smooth (singular), the problem becomes we 11 posed. ${ }^{93}$ As shown in Figure $3.1, M\left(x^{\circ}, x\right)$ is singular in our case, so numerical instabilities need not be anticipated.

Now the means of compusing the discretized version of (3.40) will be explained. First of all, the elements of $\boldsymbol{M}$ must be computed as defined by (3.37). To see what this involves, observe in Figure 3.1 that there is a logarithmic singularity in $M\left(x^{\prime}, x\right)$ in the neighborhood of $z=z_{i}$. Thus a number of the integrals in $\boldsymbol{\omega}$ are singular integrals and require special treatment. Because the B-splines are only non-zero over a finite number of breakpoint intervals, a large number of the elements of $\boldsymbol{w}$ are non-singular integrals. In order to obtain the entire matrix to machine accuracy, two special integration techniques are required. First of all, the non-singular product integration regions are computed by using Gauss-Legendre integration only between the inflection points of the B-spline $N_{i}^{m}$ involved. This strategy improves the chance that the product, $M\left(x^{*}, x\right) N_{i}^{m}$, is polynomial-like and therefore very accurately integrable by the Gauss-Legendre technique. To accurately compute the singular integrals, a new technique which essentially does an exponential transformation of the integration interval is helpful. ${ }^{30}$ The TAMCRI ${ }^{31}$ function, a FORTRAN-callable function embodies this new integration procedure. However. 
to produce a highly accurate answer, $M\left(x^{*}, x\right)$ must be available to high accuracy when $\left|z-z_{i}\right| \geqslant 10^{-13} \mathrm{~cm}$. This extremely stringent condition can only be achieved when all reducible subtractions have been removed from the computation and when $\boldsymbol{K}(k)$ and $E(k)$ can be found to high accuracy under the same conditions. Again, this latter goal is composed of two distinct phases, the computation of $k$ and then of $K$ and $E$. Because of the complexity of this subject, it is dealt with in Appendix D .

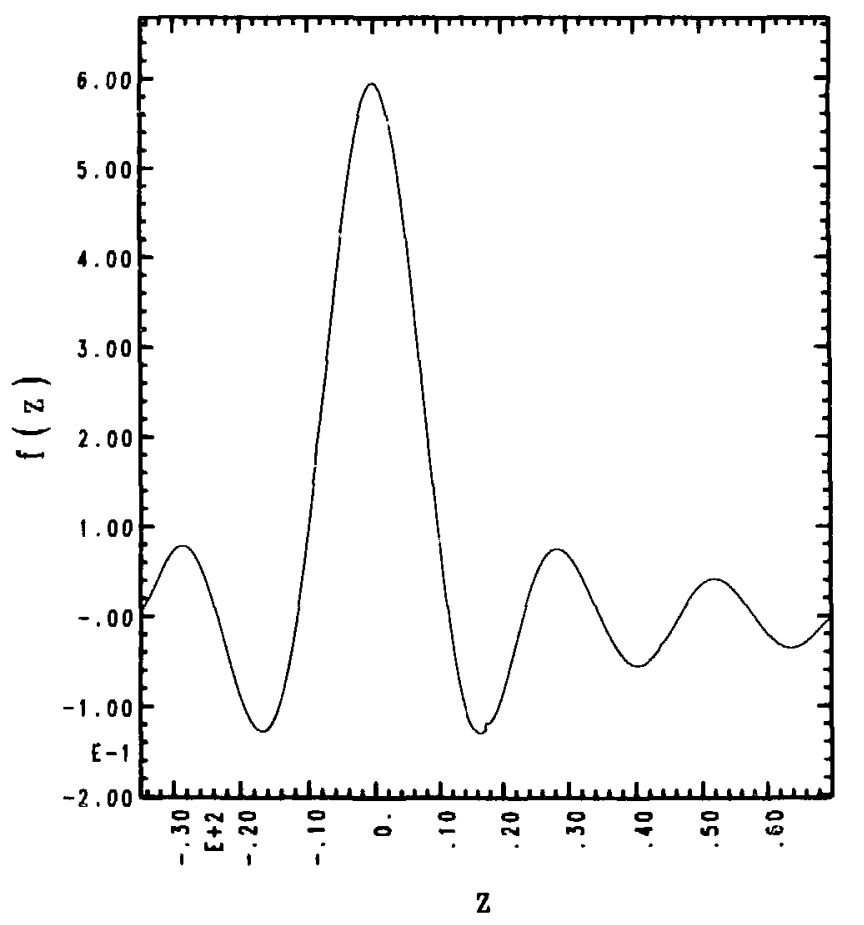

Figure 3.2. Inverted $f(z)$. Here, the original function was $2.2[\sin (.27 \mathrm{z}) / \mathrm{z}]$, and the inversion was done with quintic B-splines on four equal intervals. Collocation was performed at the Gauss-Legendre points. Note the discontinuity in $f$ at $z=17.5$. 
To this point, we have not. specified where the collocation points, $z$, are located. Of course, $\boldsymbol{M}$ is a function of their specification. I performed a series of numerical experiments for various functions $f(x)$ with quintic B-splines as basis functions. One such choice of $f$ is seen in Figure 3.2. When no continuity conditions are specified, $g(x)$ can be discontinuous at the breakpoints and six collocation points are chosen between each pair of breakpoints. Choosing the breakpoints to be evenly spaced and the collocation points to be located at the Gauss-Legendre points, $g(x)$ is found via product integration at each of the collocation points. Then, $\bullet$, an approximation to $f(x)$ at the collocation points, is found by solving (3.39). Using to define an interpolatory spline, $\hat{f}(x)$, the relative error, $|f(x)-\hat{f}(x)| / f(x)$, can be plotted. As seen in Figure 3.3, the error at the breakpoints is large as might be expected because $\hat{f}(x)$ is not required to be continuous across the breakpoints. Note also that very large errors (six orders of magnitude larger than the minimum error on the mesh) occur at other points. What is not anticipated in this experiment is that the smallest error occurs not at the Gauss-Legendre points, but at points nearby. Unfortunately, I was not able to obtain a characterization of what the optimal collocation points might be under these circumstances.

If we were to be led in our search for optimal collocation points by the results for Fredholm integral equations of the second kind, ${ }^{94}$ Volterra integral equations of the second $k$ ind, ${ }^{12}$ and $m \frac{\text { th }}{}$ order differential equations, ${ }^{7}$ we might 


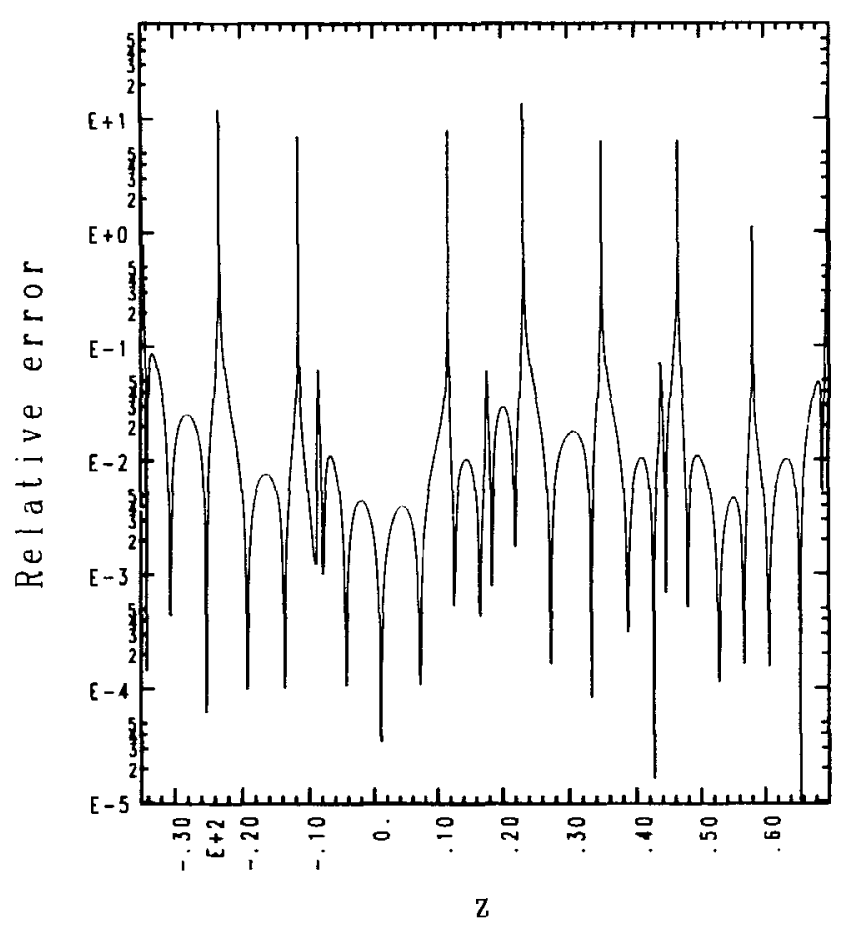

Figure 3.3. Inversion error. The inverted function was $2.2[\sin (.27 \mathrm{z}) / \mathrm{z}]$, with the inversion being done with quintic $B$-splines on four equal intervals and collocated at the Gauss-Legendre points. The points of minimum error are near the collocation points.

anticipate that Gauss-Legendre points or the breakpoints would be the best choices. Or perhaps the pessimistic result that there are no optimal collocation points for Volterra integral equations when the integrand is singular would make us similarly skeptical for first kind Fredholm integral equations. But Figure 3.3 leads us to believe that there are optimal collocation points, and that they aren't the Gauss-Legendre points (which are optimal for second kind Fredholm integral equations). The only other simple choice is to have the collocation points be the breakpoints or a combination of 
breakpoints and Gauss-Legendre points. Experiments involving this latter combination still showed large errors at the breakpoints. To use strictly the breakpoints legenerates the splir.? into a single polynomial which cannot perform the approximation well at all. Consequently. optimal collocation points remain to be discovered.

It has been argued that it is unrealistic to assess the worth of a solution technique for a class of integral equations strictly on the basis of a few test cases for $f(x) .{ }^{82}$ And the point is well taken, because if $f(x)$ has square-root singularities at $x_{1}$ and $x_{2}$, collocation at the Gauss-Legendre points performs well towards the center of the interval but poorly near the singularities. Two remedies are possible in this instance. First, the singularity can be "factored out" of the problem, 84 an analytically complex procedure requiring detailed knowledge of the character of the singularity. Secondly, and more attractively, the regions near the singularities can be treated separately with exponential or other more appropriate basis functions, and then the overall solution matched as required at the boundary between the singular and non-singular regions. This procedure has worked well in solutions of differential equations possessing boundary layers, ${ }^{39}$ but the positions of the singularities must somehow be determined before this technique is applied, a question with which we will not deal because it is akin to what is required in the AMM and AMR methods for PDEs. 


\subsection{Other Coil Integrals}

In the coil calculation, several important integrals need evaluation. For example, equation (2.139) contains a flux integral, and it has two contributions. First, $B_{\varphi}^{p}$ is integrated over the plasma region. By performing the computations implied by Appendix $\mathrm{C}$, a bi-quintic spline interpolant can be made available for any plasma variable, and simply analytically integrating this interpolant over each subdomain defined by the mesh in the plasma region yields the required value. The second contribution comes from the insulation region, or area exterior to the vacuum vessel but interior to the $\theta$-coil. To perform this integral, (2.159) must be integrated over the included region. This integration is accomplished by two-dimensional Gaussian quadrature over that area. By zoning the mesh in this region slightly more coarsely than in the plasma region, adequate numerical accuracy will be achieved. This mesh, together with the two-dimensional Gaussian quadrature scheme define the two-dimensional Gauss-Legendre points where $B_{\varphi}^{p \cdot i n s}$ must be known. Then noting that the values of $B_{\varphi}^{p}$ and its $\rho$ and $z$ derivatives can be found everywhere using the bi-quintic interpolant discussed above, the integrals in (2.159) can be evaluated by doing a two-dimensional Gaussian quadrature over each mesh-delimited subdomain in the plasma region for the appropriate values of $x$.

Another kind of integral arises in the computation of the external $B$-field in the plasma region together with its derivatives. Equations (2.185) and (2.186) are typical of the integrals that must be numerically evaluated to 
find the $\rho$ and $z$ exterinal $B-f$ ield components. As discussed in Chapter 2 , the derivatives of these components are much more complicated, but the same principles of integration apply.

Because the values of $I^{a}$ are known to highest accuracy at the collocation points on the ioroidal coils, it is important to make use of this numerical advantage and use those points in the quadrature scheme. All other quantities in the integrals can be evaluated to arbitrary accuracy. As Gauss-Legendre points are the collocation points on each interval at the present time, a one-dimensional Gaussian quadrature of the order of the number of collocation points is the method of choice. However, should other collocation points be found to produce more accurate answers for $I^{a}$, then a one-dimensional quadrature scheme accomodating the distribution of those points would need to be developed. 


\section{CHAPTER 4}

\section{TEMPORAL DISCRETIZATION AND SOLUTION METHODS}

\subsection{Model Summary}

The two spatially discretized systems which were developed in Chapter 3. (3.28) and (3.35), can be rewritten as

$$
\frac{\partial \boldsymbol{u}_{2}}{\partial t}=\boldsymbol{p}\left(\boldsymbol{u}_{2}, \boldsymbol{K}, \boldsymbol{L}, \hat{\boldsymbol{i}}, \boldsymbol{s}, \frac{d \boldsymbol{c}^{I}}{d t}\right)
$$

and

$$
\frac{d c^{I}}{d t}=\left(L^{I} p+M\right)^{-1}\left(\cdot\left(\frac{\partial U_{2}}{\partial t}\right)-R^{I} P c^{I}\right)
$$

with a set of additional spline relations on $\boldsymbol{K}, \mathcal{L}$, and $\hat{\boldsymbol{i}}$ that are effectively constraint equations and have been separated out of the system (3.28). The overali system can be symbolically represented by 


$$
\frac{\partial u}{\partial t}=\mathbf{r}\left(\boldsymbol{u}, \frac{\partial u}{\partial t}, \mathbf{s}, t\right)
$$

where $\boldsymbol{s}$ is a vector of explicit quantities. Since the argument $a_{\ell} u$ is going to be treated explicitly, equation (4.3) can be handled by standard ordinary differential equation theory.

\subsection{Implicit Temporal Integrators}

In choosing a method to solve (4.3), some brief background on linear multistep meihods (LMMs) and "one-leg" methods (OLMs) is needed. Define $a^{n}$ to denote the vector $u$ evaluated at $t=t^{n}$. Then, the operator $E$ defined by

$$
E u^{n}=u^{n+1}
$$

allows the definition of $k$-siep LMS for (4.3) to be written 5

$$
\rho(E) \boldsymbol{u}^{n}=\Delta t \sigma(E) \boldsymbol{r}^{n}
$$

where

$$
\rho(\zeta)=\sum_{j=0}^{k} a_{j} \zeta^{j} \quad, \quad \sigma(\zeta)=\sum_{j=0}^{k} \beta_{j} \zeta^{j}
$$

Consistency and normalization provide two conditions on the $\alpha^{\prime} s$ and $\beta^{\prime \prime} s$, with 
other conditions or choices determining the remaining parameters. As we are interested in solving stiff equations, i.e.. those with eigenvalues differing by orders of magnitude, an implicit A-stable technique must be used. This limits $k$ in general to $2 .{ }^{25}$ However, we are interested in more than just A-stability; we wish to se able to benefit from the new concept called "A-contractivity" which allow-i larger variations in time steps and more immunity from errors when the solution, $a$, is not smooth. ${ }^{87}$ Although there are Adam's techniques with this property (which are also "one-leg" methods), they do not possess as large a region of stability as the class of OLMs studied by Dahlquist. These OLM methods are the "twins" of the LMMs and can be written in the variable-step form as $^{26}$

$$
\rho_{n}\left(E^{\prime} u^{n}=\hbar_{n} \quad\left(\sigma_{n}(E) u^{n}\right)\right.
$$

where all the $\alpha^{\prime} s$ and $\beta^{\prime} s$ are now tims-dependent. A quick example illustrating the "twin" relationship between LMMs and OLMs is the implicit midpoint rule.

$$
u^{n+1}-u^{n}=\Delta t \quad\left(\frac{u^{n+1}+u^{n}}{2}\right)
$$

which is the "one-leg twin" of the trapezoidal rule,

$$
u^{n+1}-u^{n}=\frac{\Delta t}{2}\left(f\left(u^{n+1}\right)+f\left(u^{n}\right)\right)
$$


This last method has also been termed "Crank-Nicolsen" by some computational physicists, and is the most widely used in computational physics work because it is a second-order accurate method and is also A-stable. Unfortunately, outside ordinary differential equation circles, few have recognized that it is only marginally A-stable for the standard linear test equation,

$$
\frac{d y}{d t}=\lambda y
$$

If the differential equation turns out to be very stiff, a common difficulty in interesting physics problems, the result will be slowly damped oscillatory errors. ${ }^{05.110 .113}$ Without recognizing the source of the difficulty, Hogge $\mathrm{A}^{8}$ and Lindemuth ${ }^{72}$ observed this instability in their simulations using the. trapezoidal rule.

In real physics problems, the equations are seldom linear and of ten involve differential operators with complex discretizations. These difficulties make analysis of linearized forms of these equations either very difficult or impossible. Nevertheless, Beam and Warming ${ }^{5}$ have analyzed a parabolic test equation (instead of (4.10)) with an explicitly evaluated mixed spatial derivative. In this instance, the trapezoidal rule actually turned out to be unstable, and thereby clearly demonstrated the additional $r$ isk one might incur by using marginally stable methods. 
Since the trapezoidal rule is actually a one-step method, it makes sense to ask about other linear one-step methods, especially since they are widely used. 100 In terms of the single parameter $\theta$, they have the form

$$
u^{n+1}-u^{n}=\Delta t\left[\theta \boldsymbol{r}^{n+1}+(1-\theta) \boldsymbol{f}^{n}\right]
$$

While these methods are $A-s t a b l e$ for $1 / 2<\theta<1$, they are only first-order methods. Consequently, the most used temporal integrators are lacking in one way or another.

Turning now to second-order OLMs, Dahlquist ${ }^{26}$ gives the subset of two-step OLMs which are second-order and A-contractive. This subset is defined by the following choice of the $\alpha^{\prime} s$ and $\beta^{\prime} s$ :

$$
\begin{array}{ll}
\alpha_{n+2}=\frac{1+c}{2} & \beta_{n+2}=\frac{1+b+(a+c)}{4} . \\
\alpha_{n+1}=-c & \beta_{n+1}=\frac{1-b}{2} \\
\alpha_{n}=\frac{c-1}{2} & \beta_{n}=\frac{1+b-(a+c)}{4}
\end{array}
$$

where 


$$
\begin{gathered}
a=\frac{\left(1-c^{2}\right) t_{a}^{2}}{c}, \quad b=\left(1-c^{2}\right)\left(1-t_{a}\right)^{2}, 0<c<1 \\
\bar{h}_{n}=\frac{\left(h_{n+1}-h_{n}\right) c}{2 t_{\alpha}}, \quad t_{\alpha}=\frac{c\left(h_{n+1}-h_{n}\right)}{c\left(h_{n+1}-h_{n}\right)+h_{n+1}+h_{n}} .
\end{gathered}
$$

Here, $h_{n+1}=t^{n+2}-t^{n+1}$.

In addition to Dahlquist, Beam and Warming have characterized the A-stability region for this OLM. They did this with a very different parametrization and by doing the analysis on LMMs." The reason that the stability analysis is applicable to OLMs as well is that there is a linear equivalence between LMMs and OLMs. ${ }^{26}$ Therefore, as long as the test equation is linear. equation (4.10) or any other linear system can be used with the resill1s being equally applicable to LMMs and OLMs. The Beam and Warming parametrization $(\xi, \theta, \varphi)$ is also done for constant stepsize LMMs, and has the following two-dimensional stability region: ${ }^{5}$

$$
\theta \geqslant \frac{\xi+1}{2}, \quad \xi \geqslant-\frac{1}{2} .
$$

To compare this with Dahlquist's variable-step OLMs, his parameters must be specialized to the constant stepsize case:

$$
a=0 \quad, \quad b=1-c^{2} \text {. }
$$


Then the correspondence between the two notations can be written

$$
\xi=\frac{c-1}{2} \quad, \quad \theta=\frac{(2-c)(1+c)}{4}, \varphi=\frac{(c+2)(c-1)}{4}
$$

From equations (4.15), it is clear that Dahlquist's A-contractive techiques constitute a one-dimensional subset of the A-stable methods of Bean and Warming.

Anticipating the matrix factorization which will be used in the solution of equations (4.1), and the potential explicit treatment of $\tilde{i}$, analysis of how the stability region (4.14) is modified by these approximations is important. If the explicit terms are extrapolated to second-order. Beam and Warming found that the stability region is reduced to

$$
\theta \geqslant \frac{2(1+\xi)^{2}}{3+4 \xi} \quad, \quad \xi \geqslant-\frac{1}{2}
$$

The linear test equation used to obtain this result was a factorized, two-dimensional parabolic equation with explicit treatment of the mixed derivative term. It is naturally of interest to know how this result modifies the one-dimensional A-contractivity region. Since $\xi \geqslant-1 / 2$ implies $c \geqslant 0$, which is already required, only the first inequality will introduce any restriction. Using equations (4.16), that inequality leads to 


$$
c \leqslant \frac{1}{2} \text {. }
$$

removing one-half of the parameter space.

\subsection{Explicit Time Integration Techniques}

As noted above, the new stability results require second-order accurate extrapolation of the explicit terms, and the extrapolation point is the "one-leg" t ime.

$$
t_{O L N}=\sigma_{n}(E) t_{n}
$$

The second-order method recommended by Beam and Warming is 5

$$
\sigma_{e}(E)=\left(\xi+\frac{3}{2}\right) E-\left(\xi+\frac{1}{2}\right)=\left(\frac{c}{2}+1\right) E-\frac{c}{2} .
$$

Another complication with the explicit terms is that some of them are time derivatives such as

$$
\frac{\partial J_{p}^{p}}{\partial t}=\frac{c}{4 \pi} \frac{\partial}{\partial t}\left\{\frac{\partial B_{p}^{p}}{\partial z}-\frac{B_{z}^{p}}{\rho}-\frac{\partial B_{z}^{p}}{\partial \rho}\right\}
$$

which arises in equation (3.33). Also, since $\theta^{p}+\theta^{e x t}$, Faraday's law implies that $\partial_{i} e^{e t}$ must be available. And the fact that we have chosen to decouple the 
computation of the plasma and the external fields, equations (4.1) and (4.2). implies that this time derivative must be calculated explicitly. We choose to perform the explicit calculation of these time derivatives via a Lagrange interpolation 44 of the last three known values of $\boldsymbol{a}^{\mathrm{x} t}$. This polynomial is ther differentiated and the resulting polynomial interpolated at $t=t_{O L M}$. This sequence leads to an interpolation because the driving coils must lead in the computation. For $B_{p}^{e x t}$, for example,

$$
\begin{aligned}
\left.\frac{\partial B_{\rho}^{e x t}}{\partial t}\right|_{t=i_{O L N}}= & \left.\frac{2 t_{O L M}-t^{n}-t^{n+1}}{h_{n+1}\left(h_{n}+h_{n+1}\right)} B_{\rho}^{e x t}\right|_{t=t^{n+2}}+\left.\frac{t^{n+2}-2 t_{O L M}+t^{n}}{h_{n} h_{n+1}} B_{\rho}^{e x t}\right|_{t=t^{n+1}} \\
& +\left.\frac{2 t_{O L N}-t^{n+2}-t^{n+1}}{h_{n+1}\left(h_{n}+h_{n+1}\right)} B_{\rho}^{e x t}\right|_{t=t^{n}}
\end{aligned}
$$

Returning now to equation (4.21), since only plasma variables are involved in this computation, an extrapolation is involved, not an interpolation. In conjunction with this fact, it is assumed that the appropriate variables are known at the $n-1, n$, and $n+1$ time levels. Then the extrapolation of the time derivative of $u$ can be written

$$
\begin{aligned}
\left.\frac{\partial u}{\partial t}\right|_{t=t_{O L M}}= & \left.\frac{2 t_{O L M}-t^{n-1}-t^{n}}{h_{n}\left(h_{n-1}+h_{n}\right)} u\right|_{t=t^{n+1}}+\left.\frac{t^{n+1}+t^{n-1}-2 t_{O L M}}{h_{n-1} h_{n}} u\right|_{t=t^{n}} \\
& +\left.\frac{2 t_{O L M}-t^{n+1}-t^{n}}{h_{n}\left(h_{n-1}+h_{n}\right)} u\right|_{t=t^{n-1}} .
\end{aligned}
$$


Since the stepsize is assumed in general to be variable. it is important to see where $t_{\text {oLM }}$ lies for large increases and large decreases in this step. First of all. note that $t_{a}>0$ implies an increase in the time step, while $t_{a}<0$ implius a decrease. In the latter case, if $h_{n+l} / h_{n}$ is small, we have

$$
\begin{gathered}
t_{\alpha} \rightarrow-\frac{c}{1-c}, \quad \bar{h}_{n} \rightarrow \frac{(1-c)}{2} h_{n}, \quad a, b \rightarrow \frac{1+c}{1-c} \\
t_{O L M} \rightarrow \frac{1}{4(1-c)}\left[\left(3+2 c-c^{2}\right) t^{n+2}-4 c t^{n+1}+(1-c)^{2} t^{n}\right] .
\end{gathered}
$$

Now choose $t^{n}=0, t^{n+1}=95$, and $t^{n+2}=96$. Then, for $c=1 / 2, t_{O L M}=85$, which is an interpolation. Varying the $t^{\pi} \cdot s$ will not change this result. By letting $c \rightarrow 1$, however, $t_{O L N}$ can be made to extrapolate to infinity. But the stability region has been restricted to $0<c \leqslant 1 / 2$, so the extrapolatory behavior in this instance has been excluded.

The first case $\left(t_{a}>0\right)$ is the situation where $h_{n+l} / h_{n}$ can be large. In this limit,

$$
\begin{aligned}
& t_{a} \rightarrow \frac{c}{c+1}, \quad h_{n} \rightarrow \frac{c+1}{2} h_{n+1}, \quad a, b \rightarrow \frac{1-c}{1+c} \\
& t_{O L M} \rightarrow \frac{1}{4(1+c)}\left[\left(c^{2}+3\right) t^{n+2}+4 c t^{n+1}+\left(c^{2}+1\right) t^{n}\right] .
\end{aligned}
$$

If the time levels are chosen here to be $t^{n}=0, t^{n+1}=1$, and $t^{n+2}=100$, then again 
for $c=1 / 2, t_{O L N}=54.5$. This is an extrapolation beyond $t^{n+1}$, but not past $t^{n+2}$. The maximum that can occur in the stability region is $t_{O L N}=75(c=0)$. It appears, therefore, that the extrapolation will always be to times less than $t^{n+2}$ for large increases in stepsize.

\subsection{Fully Discretized Equations}

With these various discretization methods, equation (4.3) becomes

$$
\frac{\alpha_{n+1} u_{n+1}+\alpha_{n} u_{n}+\alpha_{n-1} u_{n-1}}{h_{n}}=f\left(\sigma_{n}(E) u_{n},\left.\frac{\partial}{\partial t}\left[\mathcal{F}\left(u_{n}\right)\right]\right|_{t=t_{O L V}}, \sigma_{e}(E) s, t_{O L N}\right)
$$

where $F$ is the Lagrange interpolation operator defined by (4.22) for the plasma equations or (4.23) for the coil equations. For the implicit calculation, there is a particularly good change of variable insofar as understanding how to compute is concerned. It is ${ }^{4}$

$$
\widehat{u}_{n}=\sigma_{n}(E) u_{n}
$$

Using this change of variable, (4.26) becomes

$$
\frac{1}{\omega \bar{h}_{n}}\left[\hat{u}_{n}-\left(\beta_{n+1}-w \alpha_{n+1}\right) u_{n+1}-\left(\beta_{n}-w \alpha_{n}\right) u_{n}\right]=r\left(\hat{u}_{n} \ldots\right)
$$

where $\omega \equiv \beta_{n+1} / a_{n+1}$. Separating of explicitly known quantities into 


$$
u_{\circ l d}=\left(\beta_{n+1}-w \alpha_{n+1}\right) u_{n+1}+\left(\beta_{n}-w \alpha_{n}\right) u_{n} \text {, }
$$

equation $(4.28)$ can be recast as

$$
F\left(\hat{u}_{n}\right)=\omega \bar{h}_{n} r\left(\hat{u}_{n}, \ldots\right)-\hat{u}_{n}+u_{o l d}=0
$$

Once equation (4.30) has been solved for $\widehat{u}_{n}$. the desired solution is obtained by $^{4}$

$$
u_{n+2}=\frac{1}{\beta_{n+2}}\left[\hat{u}_{n}-\beta_{n+1}, u_{n+1}-\beta_{n} \omega_{n}\right] \text {. }
$$

For the coil equations, (4.2), equation (4.30) implies

$$
\omega \bar{h}_{n}\left(L^{I} \boldsymbol{p}+\boldsymbol{M}\right)^{-1}\left\{\cdot\left(\left.\frac{\partial}{\partial t}\left[\mathscr{F}\left(\boldsymbol{U}_{2}\right)\right]\right|_{t=t_{0 L \alpha}}\right)-R^{I} \boldsymbol{p} \hat{\boldsymbol{c}}_{n}^{I}\right\}-\widehat{\boldsymbol{c}}_{n}^{I}+\boldsymbol{c}_{0 l d}^{I}=0
$$

where $\hat{c}_{n}^{l} \equiv \sigma_{n}(E) \epsilon_{n}^{l}$ and $c_{0 l d}^{l}$ is defined by equation (4.29). This can be more simply stated as

$$
A \hat{c}_{n}^{\prime}=b
$$

where

$$
\mathbf{A}=\left(L^{I}+\omega \boldsymbol{h}_{n} R^{I}\right) \mathbf{P}+\boldsymbol{M}
$$


and

$$
\mathbf{b}=\left(L^{I} \boldsymbol{p}+\boldsymbol{M}\right) \boldsymbol{c}_{0 l \boldsymbol{d}}^{I}+\omega \bar{h}_{n} \cdot\left(\left.\frac{\partial}{\partial t}\left[\mathscr{H}\left(\boldsymbol{u}_{2}\right)\right]\right|_{t=t_{0 L \nu}}\right)
$$

And it is clear from this analysis that solving the coil equations is a linear problem. Unfortunately, such is not the case for the plasma calculation.

In order to more clearly understand the time discretization of (4.1), we separate $\boldsymbol{p}$ as follows:

$$
\boldsymbol{p}\left(\boldsymbol{G}_{2}, \boldsymbol{s}, \boldsymbol{K}, \boldsymbol{L}, \hat{\boldsymbol{i}}, \frac{\partial \boldsymbol{q}\left(\boldsymbol{c}^{I}\right)}{\partial t}\right)=\boldsymbol{p}_{1}\left(\boldsymbol{U}_{2}, \boldsymbol{K}, \mathbf{L}\right)+\boldsymbol{p}_{2}\left(\boldsymbol{s}, \hat{\boldsymbol{i}}, \frac{\partial \boldsymbol{q}\left(\boldsymbol{c}^{I}\right)}{\partial t}\right)
$$

The intent is to treat $\boldsymbol{p}_{1}$ implicitly while $\boldsymbol{p}_{z}$ is treated explicitly. $\hat{\boldsymbol{l}}$, the vector of mixed derivative variables, has been separated out of $p_{1}$ (and therefore out of $\boldsymbol{U}_{z}$ ) because the temporal integrator stability analysis described above was done for this case, and also because this simplifies the big matrix in equation (3.28) to an essentially five-banded matrix. The key to this simplification is that when the new matrix is factorized, the two "one-dimensional" calculations which result are far more easily done. With this separation then, equation (4.30) implies 


$$
\begin{aligned}
& \omega \bar{h}_{n}\left\{\boldsymbol{p}_{1}\left(\sigma_{n}(E)\left(\boldsymbol{U}_{2}\right)_{n}, \sigma_{n}(E) \boldsymbol{K}_{n}, \sigma_{n}(E) \boldsymbol{L}_{n}\right)\right. \\
& \left.\quad+\boldsymbol{p}_{2}\left(\sigma_{e}(E) \boldsymbol{s}_{n},\left.\sigma_{e}(E) \hat{\boldsymbol{i}}_{n} \cdot \frac{\partial}{\partial t}\left[g\left(\boldsymbol{q}\left(\boldsymbol{c}^{I}\right)\right)\right]\right|_{t=t_{o l \boldsymbol{}}}\right)\right\}-\hat{\boldsymbol{U}}+\left(\boldsymbol{U}_{2}\right)_{o l d}=0 .
\end{aligned}
$$

where

$$
\hat{\boldsymbol{U}}=\sigma_{n}(E)\left(\boldsymbol{U}_{2}\right)_{n}
$$

\subsection{The Nonlinear Solver}

Because $p$, is a nonlinear operator, a nonlinear equation solver is required to find $\hat{\boldsymbol{u}}$ in equation (4.37). As the standard Newton method will not always converge in these problems, ${ }^{72}$ a more robust method is essential. Because of the cost of "probability-one" homotopy continuation methods, the "globalized" Newt on methods are used instead. ${ }^{7.111}$ In the case of equation (4.37), they can be written in the general form

$$
\begin{aligned}
\boldsymbol{F}\left(\hat{\boldsymbol{U}}_{l}\right) & =s_{l}\left[\boldsymbol{1}-\omega \hbar_{n} \boldsymbol{J}\left(\hat{\boldsymbol{U}}_{l}\right)\right]\left(\hat{\boldsymbol{U}}_{l+l}-\hat{\boldsymbol{U}}_{l}\right) \\
& =\omega \hbar_{n}\left[\boldsymbol{p}_{l}\left(\hat{\boldsymbol{U}}_{l}, \ldots\right)+\boldsymbol{p}_{2}\left(\sigma_{e}(E) \boldsymbol{8}_{n}, \ldots\right)\right]-\hat{\boldsymbol{U}}+\left(\boldsymbol{U}_{2}\right)_{0 l d} .
\end{aligned}
$$

where $\hat{\boldsymbol{v}}_{l}$ denotes the $l \underline{t h}$ iterate of the solver, $s_{l}$ is the (signed) stepsize at the $l \underline{t h}$ iteration, and 


$$
\left.\left[\boldsymbol{J}\left(\hat{\boldsymbol{U}}_{l}\right)\right]_{i j} \equiv \frac{\partial\left(\boldsymbol{p}_{1}(\boldsymbol{u})\right)_{i}}{\partial \boldsymbol{u}_{j}}\right|_{\boldsymbol{u}=\hat{\boldsymbol{v}}_{l}}
$$

Hirsch and Smale ${ }^{4}$ present a series of Newton-like algorithms fitting the general framework of equation (4.39), but the determination of $s_{l}$ is complicated. In our context, what they call the "Newton-vector" is defined by

$$
M(\hat{U})=\operatorname{sgn}\left(\operatorname{det}\left[\omega \hbar_{n} J(\hat{U})-1\right]\right)\left[\omega \hbar_{n} J(\hat{U})-1\right]^{-1} F(\widehat{U})
$$

This vector is found first of all by solving the problem

$$
\left[\omega \hbar_{n} \boldsymbol{J}(\hat{\mathbf{U}})-1 j \cdot=\boldsymbol{F}(\hat{\boldsymbol{U}})\right.
$$

for . It is of great importance that the sign information required in equation (4.41) can also be obtained when - is found. Then, noting that the signed term does not affect the Euclidean norm, the norm of the "Newton-vector" can be found as follows:

$$
|m(\hat{U})|=|\bullet(\hat{U})|
$$

With this, they define the "Newton step of length $\rho$ from $\hat{\boldsymbol{\sigma}}$ ' to be

$$
T_{p}(\hat{U})=\hat{U}+\frac{\rho}{|M(\hat{U})|} N(\hat{U})
$$


where $\boldsymbol{T}$ is called the "Newton transformation" mapping. Hirsch and Smale's theory shows that when $\left|\boldsymbol{F}\left(\hat{\boldsymbol{U}}_{l}\right)\right|$ is decreasing for a series of iterates.

$$
\operatorname{det}\left[\omega \hbar_{n} \boldsymbol{\partial}(\hat{\boldsymbol{U}})-1\right]>0
$$

Also in this case, their Algorithm $B^{\prime}$ proceeds by standard Newton-Raphson iteration $\left(s_{l}=1\right)$ :

$$
\hat{u}_{l+1}=\hat{u}_{l}+M\left(\hat{u}_{l}\right)
$$

In other words, their algorithm senses when it is in the domain of attraction of the zero of $\boldsymbol{F}(\hat{\boldsymbol{u}})$ and switches over to the quadratically convergent Newton-Raphson technique in that instance. Otherwise, it uses the appropriate number of iterates of the Newton transformation, $\boldsymbol{T}$, and a variable (decreasing) step length $\rho$ to find the region where condition (4.45) holds. The termination of the algorithm follows the standard procedure for terminating a Newton-Raphson iteration, i.e., when $\left|\boldsymbol{F}\left(\hat{\boldsymbol{U}}_{l+l}\right)\right|<\varepsilon$, where $\varepsilon$ is some predetermined small quantity.

While this procedure appears to have the potential to be expensive, some economies could conceiveably be introduced by using quasi-Newton methods which do not require that the full Jacobian matrix be computed at every step. While there is no difficulty with this (if the Jacobian fits in the computer's memory) in the Newton-Raphson steps, the potential for condition (4.45) to hold at one 
iterate and not at the next when the iterates of $\boldsymbol{r}$ are being computed requires some finesse which is not currently built into the quasi-Newton strategies.

Another potentially very serious drawback of Hirsch and Smale's algorithm is that when it encounters singular Jacobians, it must take very small steps to get past the singularity. ${ }^{58}$ in response to this potential problem. Keller 58 proposes a method called "pseudo-arclength continuation." While it involves several very expensive procedures for large systems of equations, Keller claims they can nonetheless be approximated cheaply. It is not clear at this writing how well the sparsity pattern of the current Jacobian could be exploited in his method. Moreover, because of the usual diagonal dominance of

$$
1-\omega \hbar_{n} J
$$

it is not anticipated that singularities will be frequently encountered. However, experience may prove this point of view to be wrong.

The system of equations represented by (4.39) is indeed very large, so large in fact that even sparse matrix solvers cannot solve it efficiently on current computers. One standard means of bringing the problem within reach is to approximate it via the matrix factorization

$$
s_{l}\left[1-\omega \hbar_{n} J_{x}\left(\hat{U}_{l}\right)\right]\left[1-\omega \hbar_{n} J_{x_{2}}\left(\hat{\boldsymbol{U}}_{l}\right)\right]\left(\hat{\boldsymbol{U}}_{l+1}-\hat{\boldsymbol{U}}_{l}\right)=F\left(\hat{\boldsymbol{U}}_{l}\right)
$$

Here, $\boldsymbol{J}_{\boldsymbol{x}}$, and $\boldsymbol{z}_{x_{2}}$ denote a symbolic splitting of $\boldsymbol{s}$. All that is really 


$$
\boldsymbol{J}=\boldsymbol{J}_{x_{1}}+\boldsymbol{J}_{x_{2}}
$$

The error introduced by the factorization is

$$
-\omega^{2} \hbar_{n}^{2} \boldsymbol{J}_{x_{1}}(\hat{\boldsymbol{U}}) \boldsymbol{J}_{x_{z}}(\hat{\boldsymbol{U}}) \sim \mathcal{O}\left(\hbar_{n}^{2}\right) \sim \mathcal{O}\left([\Delta t]^{2}\right)
$$

where $\Delta t=\max \left(h_{n}, h_{n+t}\right)$. This implies that the left-hand side of equation (4.48) is second-order accurate. But $\boldsymbol{F}(\hat{\boldsymbol{U}})$ already represents a second-order accurate temporal approximation to the original problem, so (4.48) can be viewed as the Newton solver for a slightly different problem, but one which is entirely equivalent to order $(\Delta t)^{2}$. This is important because, if the Hirsch-Smale algor thm is used, the order $(\Delta t)^{2}$ deflection from the Newton path caused by the factorization must not be large enough to invalidate

$$
\begin{aligned}
& \operatorname{sgn}\left(\operatorname{det}\left[\omega \hbar_{n} \boldsymbol{J}\left(\hat{\boldsymbol{U}}_{l}\right)-\boldsymbol{1}\right]\right)= \\
& \quad \operatorname{sgn}\left(\operatorname{det}\left[\omega \hbar_{n} \boldsymbol{J}_{x_{1}}\left(\hat{\boldsymbol{U}}_{l}\right)-1\right] \operatorname{det}\left[\omega \bar{h}_{n} \boldsymbol{J}_{x_{2}}\left(\hat{\boldsymbol{U}}_{l}\right)-1\right]\right) .
\end{aligned}
$$

While pathological cases may invalidate (4.51), it would certainly seen that the second-order equiralence of the two problems should be enough to make it hold under nearly all condiijons the Hirsch-Smale algorithm might encounter. 
In terms of $(3.28)$, we now choose $\boldsymbol{J}_{x_{1}}$ and $\boldsymbol{J}_{x_{2}}$ to be

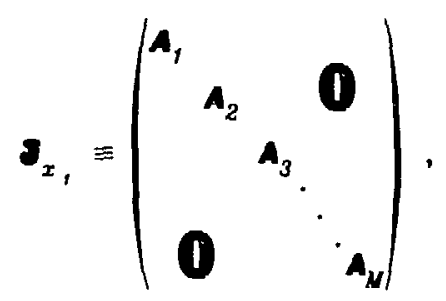

and

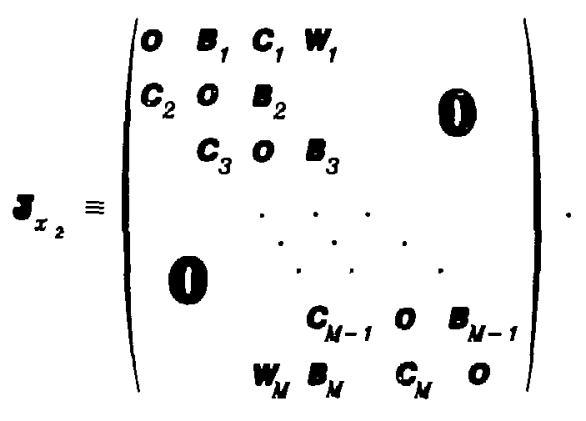

Because $\hat{\imath}$ is now being treated explicitly, the $\boldsymbol{b}_{i}^{\prime} \mathbf{s}$ and $\boldsymbol{c}_{i}$ 's are all block-diagonal matrices. Thus, the "factored" problem can be solved in two steps. They are

$$
s_{l}\left[1-\omega \hbar_{n} g_{x}\left(\hat{U}_{l}\right)\right] \geq=F\left(\hat{U}_{l}\right)
$$

followed by

$$
\left[1-\omega h_{n} J_{x_{2}}\left(\hat{U}_{l}\right)\right]\left(\hat{u}_{l+1}-\hat{U}_{l}\right)=\Omega
$$


By virtue of the structure of $\boldsymbol{J}_{\boldsymbol{x}}$, the first step involves $M$ independent systems of linear equations. While a little harder to recognize, the second step involves $N$ independent systems of linear equations, where each matrix in $\boldsymbol{J}_{\boldsymbol{x}_{2}}$ contributes one block matrix to each of the $N$ systems. It is important to note that the spline equations for $\boldsymbol{K}$ are associated with the first step and double the size of each basic block matrix involved. Similarly, the spline equations for $L$ are associated with the second step and also double the size of the basic block matrices there. Alternatively, it has been suggested that the $\boldsymbol{K}$ and $\boldsymbol{L}$ spline equations be solved explicitly after equations (4.54) and (4.55) have been solved and the entire system iterated. Although this idea may have merit, it is not pursued further here.

\subsection{Tridiagonal Solver}

The basic structure of each linear system in equations (4.54) and (4.55) is the same; it is given by matrix (3.29). While a tridiagonal solver has been given for (3.29) with $\boldsymbol{a}_{1}=\boldsymbol{a}_{N}=0$, none has been developed for the case where they are not zero. Following Hindmarsh, ${ }^{48}$ we do an $L U$ decomposition of (3.29): 


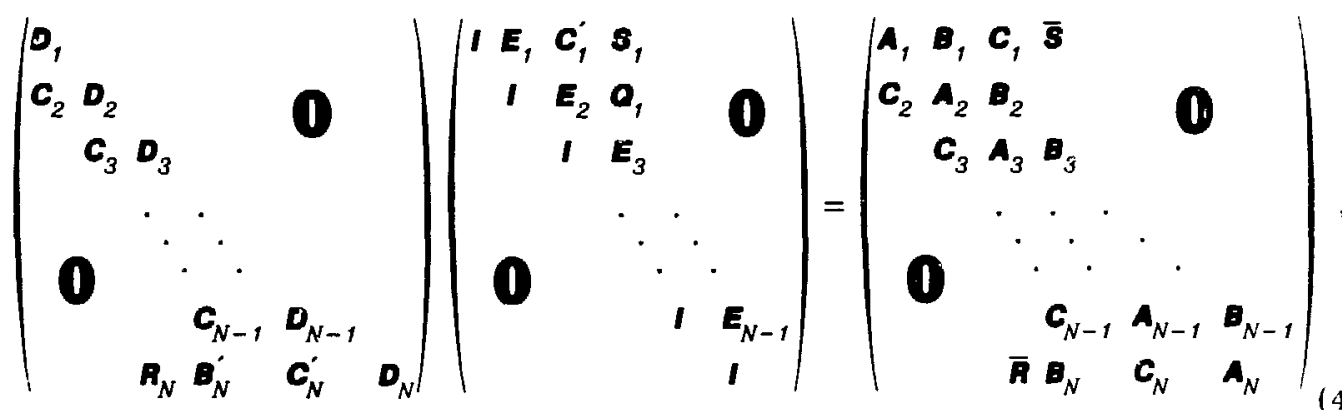

where the last matrix is (3.29) with new names for the block entries. By performing the matrix product in equation (4.56) and comparing block by block with the right-hand side, the procedure for computing the elements of the lower block matrix and the upper block matrix can be deduced. It is:

$$
\begin{aligned}
& D_{1}=A, \quad, \quad E,=D_{1}^{-1} B_{1}, \quad C_{1}^{\prime}=0_{1}^{-1} C_{1}, \quad s_{1}=D_{1}^{-1} \bar{s} \\
& D_{2}=A_{2}-C_{2} E_{1} \quad, \quad E_{2}=D_{2}^{-1}\left[B_{2}-C_{2} C_{1}^{\prime}\right] \quad, \quad Q_{1}=D_{2}^{-1} C_{2} s_{1} \\
& D_{3}=A_{3}-c_{3} E_{2} \quad, \quad E_{3}=D_{3}^{-1}\left[B_{3}-c_{3} Q_{1}\right] \\
& D_{k}=A_{k}-C_{k} E_{k-1} \quad, \quad E_{k}=D_{k}^{-1} \boldsymbol{B}_{k} \quad(k=4,5, \ldots, N-1) \\
& \boldsymbol{h}_{N}=\overline{\boldsymbol{A}} \quad, \quad \boldsymbol{B}_{N}^{\prime}=\boldsymbol{B}_{N}-\boldsymbol{A}_{N} E_{N-3} \quad, \quad \boldsymbol{C}_{N}^{\prime}=\boldsymbol{C}_{N}-\boldsymbol{B}_{N}^{\prime} E_{N-2} \quad, \quad \boldsymbol{D}_{N}=A_{N}-\boldsymbol{C}_{N}^{\prime} E_{N-1}
\end{aligned}
$$

The solution steps then read

$$
\begin{aligned}
& \mathbf{z}_{1}=\boldsymbol{D}_{1}^{-1} \mathbf{y}_{1} \\
& \mathbf{z}_{k}=\boldsymbol{D}_{k}^{-1}\left[\mathbf{y}_{k}-\boldsymbol{C}_{k} \mathbf{z}_{k-1}\right] \quad(k=2,3, \ldots, N-1) \\
& \mathbf{z}_{N}=\boldsymbol{D}_{N}^{-1}\left[\mathbf{y}_{N}-\boldsymbol{C}_{N}^{\prime} \mathbf{z}_{N-1}-\boldsymbol{\theta}_{N}^{\prime} \mathbf{z}_{N-2}-\boldsymbol{R}_{N} \mathbf{z}_{N-3}\right],
\end{aligned}
$$

followed by 


$$
\begin{aligned}
& \boldsymbol{x}_{N}=\boldsymbol{x}_{N} \\
& \boldsymbol{x}_{k}=\boldsymbol{z}_{k}-\boldsymbol{E}_{k} \boldsymbol{x}_{k+1} \quad(k=N-1, N-2, \ldots, 3) \\
& \boldsymbol{x}_{2}=\boldsymbol{x}_{2}-\boldsymbol{E}_{2} \boldsymbol{x}_{3}-\boldsymbol{O}_{1} \boldsymbol{x}_{4} \\
& \boldsymbol{x}_{1}=\boldsymbol{x}_{1}-\boldsymbol{E}_{1} \boldsymbol{x}_{2}-\boldsymbol{C}_{1} \boldsymbol{x}_{3}-\mathbf{S}_{1} \boldsymbol{x}_{4} .
\end{aligned}
$$

In the solution steps, the $y_{2}$ vectors make up the $r i g h t-h a n d s i d r$ lector of

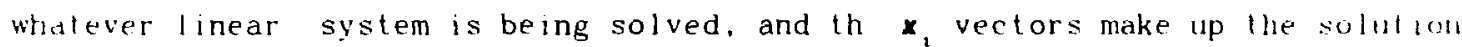
vector of that system.

There are alternatives to this method of solution of the tridiagonal system. In partjcular. Kershaw ${ }^{7}$ and Wang ${ }^{12}$ of fer excellent allernatives for solving scalar tridiagonal systems on parallel processors. However. hecallse there are a large number of identically-structured block tridiagonal systems to solve in equation (4.54) as well as in equation (4.55). l elect to parallelize the computation over the block tridiagonal systems. Some FORTRAN functions written for this purpose by A. Creenbaum were modified to handle the more general matrix structure of (3.29), and a highly-parallel and efficient solution technique for (4.48) was the result. Since setting $s_{l}=1$ in equation (4.48) gives an equation that can be solved to find in equation (4.42), all of the necessary information for the Hirsch-Smale algorithm is available via this solution technique. 


\section{CHAPTER 5}

\section{AUTOMATIC CODING OF THE PHYSICAL MODEL}

\subsection{Introduction}

The ultimate goal of those who develop big physics codes is to be able to give to a computer algebra system the physical model specifications of their problem, together with the details of discretization and solution methods, and have an optimized program to solve that physical model appear as the output of the algebra system. For the moment, this sort of dream-world situation is just that, pie-in-the-sky. However, major parts of this very difficult task have been achieved for the discretization and solution methods described in Chapters 3 and 4. The automatic coding is largely independent of the physical model, provided partial differential equations are not replaced by integro-differential equations, complications which can alter the validity or appropriateness of the discretization techniques which are "built in." For example, the physical model of the coil equations is sufficiently different in character from the model 
describing the plasma, that separate algebra facilities were developed to discretize the integro-differential systems of equations for the $I$ and $C$-coils.

As described in Chapler 1. there have been a number of attempts to automate the algebraic work. Except for the work of Wirth, ${ }^{1}{ }^{7}$ these have all involved simple one-dimensional systems of PDEs. Even in Wirth's work, however. a number of substantial difficulties kept his techniques from working on even a modest set of PDEs. The means for overcoming these obstacles, and others which appear as one tackles problems of vastly greater complexity, is the subject of this chapter.

\subsection{The Primary Obstacles}

In his atlempt to generate "black box" techniques to handle all finite-difference-type discretization methods for PDEs, Wirth experienced immediately the "intermediate expression swell" phenomenon common to all algebraic processing work. This severely restricted the size of problem he could process on the "limited-memory" DEC-10 computer running MACSYMA at MIT. Consequently, he never ran into the second primary obstacle to large-scale algebraic processing, that of excessively large consumption of computer time In fact, both the memory and processor time used by many important algebra functions tend to grow exponentially while the size of the expressions operated on grows only linearly. 
With an extremely large partial differential equation model such as the one given in Chapter 2, these problems are exacerbated to the point that the model could not be processed in any reasonable length of time, and intermediate results generated from it would not $f$ it on even the largest currently-available virtual memory computer ( $\sim 50 \mathrm{mill}$ ion words of computer memory).

There are at least six separate means of circumventing these difficulties, some being novel and others restatements of common sense approaches to these problems. They are: first, a reduction of the algebraic form of the physical problem statement to its very simplest form. Second, use of the most compact and efficient internal representation of the host algebra!c processing system. Third, structuring of the results at every step to facilitate "divide and conquer" strategies. Fourth, disposing of all "excess baggage" (via garbage collection) at the earliest feasible point in the calculation. Next, critical optimization and factoring capability must be made to run comparatively "fast." Also, other much used functions which are slow may need recoding. And finally, for the very large-scale physical model in question here, using a virtual memory computer.

In addition, there are other major concerns, depending on the capability of the algebra system to be used. As stated earlier, the fact that no major progress in automating the development of physics codes has previously occurred is strong evidence that the algebra systems themselves are deficient or insufficiently developed. However, there are certain key capabilities found 
only in MACSYMA which made this'system the one of choice. In particular, the abllity to translate MACSYMA level code into MACLISP, a LISP dialect in which MACSYMA is written. and then compile the LISP code into highly efficient machine code made a substantial difference in the execution time of the program which generated the final FORTRAN code. Also of great importance efficiency-wise were two key MACSYMA functions. First. APPLY_NOUNS is a very efficient function allowing so-called "NOUN" forms in MACSYMA to be evaluated, but it does so in a manner which avoids the substantial overhead of the system's evaluation function, EV. This efficiency gain is important in expanding out derivatives in the process of transforming the initial partial differential equations into "convective form." The second MACSYMA function of great importance in this context is SUBLIS. With this function, substitutions for a large number of scalar variables can be made in parallel into a target expression. Note, then, that these functions really $f$ it into the fifth category listed above.

\subsection{Reduction to Simplest Possible Terms}

Most of the obstacles to keeping statement of the physical problem in simplest possible form stems from the desire to be able to solve as many physics problems with the final FORTRAN code as possible. Automatic generation of code segments will, however, make this point of view obsolete as soon as it becomes very economical to generate these segments. The fact that the computer run 
whicn generated the code for the problem in Chapters 2, 3, and 4 cost only a few thousand dollars indicates that that day is not $f$ ar of $f$

As a preliminary note to the examples which follow, the meaning of MACSYMA interactive input and output symbols should be explained. The lines below which begin with $(C n)$ where $n$ is an integer contain, on the remainder of those lines, input information to MACSYMA. On the other hand, the lines beginning with $(D n)$, where again $n$ is an integer, contain on that line (and possibly previous and subsequent lines) output from MACSYMA.

The first and most obvious example of unneeded generalization consists in treating dependent variables in a two-dimensional calculation as though they depended on all three coordinate directions. This leads to much larger expressions than are actually required. As an example, consider the dependent variable $F$. In MACSYMA, we would want to declare its dependencies as

(C1) depends $(F,[z 1, z 3])$;

$$
[F(21, Z 3)]
$$

not as in the declaration

(C2) depends $(F,[z 1, z 2, z 3])$;

$$
[F(Z 1, Z 2, Z 3)]
$$


The physical model should be restricted to the one which is of real interest. Additional terms can be treated in a "post-processing" mode. although this capability has not been specifically developed, and some sacrifice of optimization in the FORTRAN output would result.

The next important area of problem simplication is that of selection of coordinate system. While some examples may require more than one coordinate system in a given physics code, it is generally true that only one coordinate system is required to study a given problem. Again, given that the automating of the algebra and code generation will one day be cheap. a given physical model should be processed in each coordinate system of interest. The use of non-orthogonal coordinate systems in AMM solution methods complicates this whole discussion. The vital point here would be to take advantage of any partial orthogonality available, in a two-dimensional problem for example.

To make this issue clear for the gase of orthogonal coordinate systems, consider the following example, where $D I V$ is the divergence operator in generalized orthogonal coordinates, and its argument is a list of the components of the vector $\boldsymbol{g}$ :

(C1) $x: \operatorname{div}([g 1, g 2, g 3])$;

(D1)

$$
\frac{\frac{d}{d X 1}(G 1 H 2 H 3)+\frac{d}{d X 2}(G 2 H 1 H 3)+\frac{d}{d \times 3}(G 3 H 1 H 2)}{H 1 H 2 H 3}
$$

Assuming that the components of $g$ and the scale factors all depend on all three 
generalized coordinates, $x 1, x 2$, and $x 3$, this expression can be expanded into

(C2) apply_nouns $(x)$;

(D2) $\left(G 2 H 1 \frac{d H 3}{d X 2}+G 1 H 2 \frac{d H 3}{d X 1}+G 1 \frac{d H 2}{d X 1} H 3+\frac{d G 1}{d X 1} H 2 H 3+G 2 \frac{d H 1}{d X 2} H 3\right.$

$$
\left.+\frac{d G 2}{d X 2} H 1 H 3+G 3 H 1 \frac{d H 2}{d X 3}+G 3 \frac{d H 1}{d \times 3} H 2+\frac{d G 3}{d X 3} H 1 H 2\right) /(H 1 H 2 H 3)
$$

Specializing to spherical coordinates, on the other hand, leads to

(C3) $x$ : sublis $([x 1=r, x 2=t h, x 3=p h i, h 1=1, h 2=r, h 3=r * \sin (t h)], x) \$$

(C4) Expand(apply_nouns $(x))$;

(D4)

$$
\frac{G 2 \operatorname{Cos}(T H)}{R \operatorname{SIN}(T H)}+\frac{\frac{d G 3}{d P H I}}{R \operatorname{SIN}(T H)}+\frac{\frac{d G 2}{d T H}}{R}+\frac{2 G 1}{R}+\frac{d G 1}{d R}
$$

This last result of five terms, as compared to the generalized coordinate representation of nine terms, is far more compact, resulting in a memory savings of roughly $50 \%$.

Another inappropriate generalization when the physical model is nonlinear is to discretize the problem in an arbitrary set of basis functions. The reason for this is that a Jacobian matrix is required for solution techniques in the nonlinear case, and, in general, higher-order basis functions tend to increase the bandwidth of that matrix. For example, $7 \underline{\mathrm{h}}$ order B-splines lead to two more non-zero elements per row in the Jacobian than do quintic B-splines. So, if the intent is to actually use quintic B-splines in production: 
Iuns, a lery large amount of additional algebraic computation would be performed, perhaps needlessly. Most critical here, however, is the potential that these additional terms have for causing the overall computation to run out 1) memory. This is particularly true in the Jacobian calculation because the differentiation process can sometimes proliferate to an exceedingly large number of terms.

A final area where generality can be an excessive burden involves tine solution technique. Whereas in Chapter 4, we chose a specific matrix splitting approach to render the solution process tractable, it is possible to use similar alternating direction implicit (ADI) schemes which involve time splitting. lt is further possible to solve the entire problem via the incomplete Cholesky conjugate gradient (ICCG) technique or one of its variants with the mixed derivative terms treated implicitly. Or, each model equation can be time-split from the others and solved separately via an ICCG technique.

To generate the Jacobian required for each possibility would lead to algebraic computations which might never be used, jusi as in the case of the basis functions. Consequently, the algebra should be performed only for the specific solution technique which is to be used. 


\subsection{Efficient Internal Representations}

The discretization process of Chapters 3 and 4 yields a computational mesh on which all variables must be defined. It is thus natural to subscript these variables according to the mesh point where they are being evaluated. The next logical step is to represent these subscripted variables algebraically as subscripted variables too. However, this is not the correct thing to do. To understand why, it is essential to understand that the use of a subscripted or array-type variable in an algebra processing system implies that the elements of that variable are assigned to possible different expressions. That this is not the intent here was explained in Chapters 3 and 4. There, the discretized equations are formally identical everywhere in the interior of the domain. Boundary equations on the left boundary lead to a number of discretized equations on that boundary which are again formally identical to one another. Similar statements can be made concerning each of the other boundary surfaces. Therefore, only a few formally different equations describe the discrete version of the problem. And while in principle this overall system of equations (multivariate polynomial equations) can be solved, it is in practice never done because the algebra could not be done on the fastest of today's computers in any finite length of time even if the closed-form solution could be found. In fact, that is the reason for this work: to algebraically compute all of the information necessary to perform the calculation numerically. 
Hence, the formal subscripts appearing in the discretized equations are to be treated formally as weil. As one possibility, the subscript is carried in the name of the variable. For example. for the electron temperature at the $i, j$ th grid point, we could choose to do:

(C1) $t e \backslash(i \backslash, j \backslash)$;

$$
T E(I, J)
$$

instead of the subscripted MACSYMA quantity

(C2) $t e[i, j]$;

(D2)

TE $I, J$

In the first case, the variable is now an "ATOM" instead of an "ARRAY" variable. Because of the nature of the implementation of MACSYMA, this uses far less storage. "ATOM"s also involve less time overhead in typing tests where they are usually the first set of objects to be categorized by internal MACSYMA functions.

Another internal representation which tends to be inefficient is that for derivatives. Again, "ATOM"s are chosen to replace derivative forms, but this cannot be done until the expansion of derivatives is effected. Also, spatial derivatives of dependent variables are handled specially, reflecting the discussion in Chapter 3. But first, let's consider derivat:ves of the transport variables. By defining the dependencies of each transport variable, the 
derivative expansion process can operate in two possible modes. First, if the DEPENDS function is used to set up the dependency, the result is strictly formal :

(C1) depends $(t e, z 1)$ :

(D1)

$[T E(Z 1)]$

(C5) depends (alpha_parallel,te):

[ALPHA_PARALLEL(TE)]

(C6) diff(alpha_parallel,z1);

$(D 6)$

$\frac{d A L P H A \_P A R A L L E L}{d T E} \frac{d T E}{d Z 1}$

On the other hand, it is possible to have the derivative form automatically replaced by an "ATOM" with mnemonic significance:

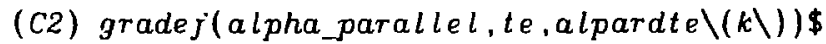

(C3) diff(alpha_parallel,z1);

(D3)

$A L P A R D T E(K) \frac{d T E}{d Z 1}$

Here $A: A A R D T E(K)$ is intended to be a vector quantity in the target FORTRAN code, so the formal subscript " $(K)^{\prime \prime}$ is concatenated to the end of the name. It is intrinsically implied that a means for compuling this FORTRAN vector is otherwise provided. 
To illustrate how the case of derivatives of dependent variables is handled, the quintic spline approximation for the second derivative of $T_{e}$ with respect to $x$, at the $i, j \underline{\text { th }}$ grid point can be written

$\frac{\partial^{2} T E(I, J)}{\partial \times 1^{2}} \longrightarrow K_{i, j}^{T}+\frac{h_{j}^{3}+h_{j+1}^{3}}{6 h_{j+1}\left(h_{j}+h_{j+1}\right)^{2}}\left[K_{i+1, j}^{T}-\left(1+\frac{h_{j+1}}{h_{j}}\right) K_{i, j}^{T}+\frac{h_{j+1}}{h_{j}} K_{i-1, j}^{T}\right]_{(5.1)}$

This substitution leads to a large increase in the size of the expressions containing the derivatives, so we choose instead to make the substitution

$$
\frac{\partial^{2} T E(I, J)}{\partial X 1^{2}} \longrightarrow \operatorname{TED} 2 X 1(K)
$$

where gradient properties are established for TED2X1(K) so that the correct behavior in the Jacobian calculation is maintained.

A final category where the internal representation can have important consequences consists of information which is derived from operations such as the above derivative associations. This information is represented by list structures which reflect the basic character of the information and makes it rapidly accessible and compactly representable. For example, the spatial derivative replacement process leads to the need to maintain extra gradient information for the Jacobian calculation as mentioned above. However, to keep the evaluation of derivatives optimal, the MACSYMA gradient database must be 
kept as small as possible. Consequently, the extra gradient information is added only just before the Jacobian is to be computed.

\subsection{Structuring of Results at Every Step}

The need to structure the equations and intermediate expressions is obvious if one is aware that most factoring and expansion routines are possibly the worst offenders in behaving exponentially more slowly as a function of expression size. Thus, if the model does not provide us with natural structuring of the equations, some artificial choices may be necessary for the sake of efficiency in the code generation process. One example of this arises in the definition of some transport quantities where, among others, $x_{e}$ and $x_{2}$ are chosen as subsidiary variables. These variables have only numerical significance and could be dispensed with. However, these variables help make the computation of Jacobian-related quantities for these transport variables much more efficient for the same reason that in derivative replacement, it is a better idea to do that replacement with a MACSYMA "ATOM" which has symbolic significance than with the actual expression used to compute it numerically.

Another example is $B$. Here the variable does have physical meaning but is not essential to the equations themselves. That is, $B$ could be replaced by its definition,

$$
\sqrt{ }\left(B_{x_{1}}^{\prime 2}+B_{x_{2}}^{\prime 2}+B_{x_{3}}^{\prime 2}\right)=\sqrt{ }\left[\left(B_{z}^{p}+B_{z}^{e x t}\right)^{2}+\left(B_{\rho}^{p}+B_{\rho}^{\rho x t}\right)^{2}+\left(B_{\theta}^{p}+B_{\theta}^{e x t}\right)^{2}\right]
$$


everywhere it appears in our defining equations. Some of the derivatives of this quantity required by the Jacobian calculation are:

$$
\frac{\partial B}{\partial B_{\rho}^{p}} \cdot \frac{\partial^{2} B}{\partial\left(B_{\rho}^{p}\right)^{2}} \cdot \frac{\partial^{2} B}{\partial B_{\rho}^{p} \partial B_{\theta}^{p}} \cdot \frac{\partial^{2} B}{\partial B_{\rho}^{p} \partial B_{z}^{p}}, \frac{\partial B}{\partial B_{\theta}^{p}}, \text { etc. }
$$

These derivatives give $r$ ise to larger expressions, and a perusal of the MHD equations shows that the derivatives occur frequently enough to cause substantial expansion when a partial derivative is applied. Because powers of $B$ reappear in every partial derivative of $B$, the solution to the growth problem here is clear. Some typical examples are:

$$
\begin{aligned}
& \frac{\partial B}{\partial B_{\rho}^{p}}=\frac{B_{\rho}^{p}+B_{\rho}^{e x t}}{B} \\
& \frac{\partial^{2} B}{\partial\left(B_{\rho}^{p}\right)^{2}}=\frac{\left(B_{\rho}^{p}+B_{\rho}^{e x t}\right)^{2}-B^{2}}{B^{3},} \\
& \frac{\partial^{2} B}{\partial B_{\rho}^{p} \partial B_{\theta}^{p}}=-\frac{\left(B_{\rho}^{p}+B_{\rho}^{e x t}\right)\left(B_{\varphi}^{p}+B_{\varphi}^{e x t}\right)}{B^{3}} .
\end{aligned}
$$

These two examples are not really artificial structuring choices, but illustrate a means of imposing a layering on the physical system. For example, transport variables in general could be defined as those variables whose definition is not found in the PDEs, but one layer down in the system. 
The final example is a truly artificial one. The vector quantity, $A$, occurs in two places in the MHD equations. More importantly, application of the derivative operators in $\boldsymbol{A}$ results in a tremendous expansion in expression size. Futher expansion of the component expressions for the purpose of restructuring each component into convection form leads to a huge expression and therefore an exponentially slow computation. The solution is to break $\boldsymbol{R}$ up into smaller pieces and individually process these pieces. Specifically, we chose:

$$
A=\alpha s, \quad s=s_{1}+s_{2}+s_{3}+s_{4}+s_{5}+s_{6}+s_{7},
$$

where

$$
\begin{aligned}
& \boldsymbol{s}_{1}=\left(\frac{e Z \rho f_{i}}{m_{i}}\right)^{2} \eta_{N} u \quad \boldsymbol{s}_{4}=\alpha_{\wedge} \frac{\Delta \times u}{B} \\
& \boldsymbol{s}_{2}=\left(\alpha_{+}-\alpha_{\|}\right) \frac{\boldsymbol{\theta} \cdot \boldsymbol{u}}{B^{2}} \quad \boldsymbol{s}_{5}=\left(\beta_{+}-\beta_{\|}\right) \frac{\boldsymbol{B} \cdot \boldsymbol{\nabla} T_{e}}{B^{2}} \boldsymbol{B} \\
& \boldsymbol{s}_{3}=-\alpha_{4} u \quad \boldsymbol{s}_{6}=-\beta_{+} \nabla T_{e} \\
& \boldsymbol{s}_{\gamma}=-\beta_{\wedge} \frac{\operatorname{E\nabla } T_{e}}{B}
\end{aligned}
$$

While some restructuring such as that done on $\boldsymbol{A}$ could be done automatically, no sof tware was written to perform the task. 
On the other hand, a natural structuring framework comes from the definition of "convective form" for example. Also, in the initial PUEs, terms mat be grouped together and multiplied by a common constant if lhey are physically of the same character. Consequently, a means of processing the pieces of these structured forms of the initial PDEs instead of the flDEs II the wentrety is essential. A MACSMMA function was developed specifically to offect this piece by piece processing of the PDEs into convective form. It is called PROCESS_PDE_STRUCTURE.

After this great amount of effort has been expended to break the initial equations into small enough pieces so that some intermediate form of the expressions can be computed in a finite length of time, care must be taketi to keep these results in as structured and compact a form as possible. This becomes more difficult because most structuring at this point will be contrived. An exception would be keeping that part of the PDEs which is to be treated explicitly in the nonlinear calculation separated from the rest of the terms in the transformed PDEs until the Jacobian calculation has been completed. Other contrived techiniques will simply involve making arbitrary decisions as to how to break expressions into pieces so as to make the overall computation proceed more rapidly. 


\subsection{Elimination of Excess Baggage}

For the most part, eliminating all unneeded expressions, lists, and so forth is a programming style which is second-nature to people who program in LISP and other Ianguages that allow "block" structuring with local binding mechanisms. In MACSYMA, for instance, if a variable is bound to some object in a local sense within a block and perhaps modified within the scope of that block, the variable is garbage-collected when the program proceeds outside the scope of the block. This process frees the memory occupied by that variable for future use.

While this programing style is sufficient in essentially all cases, it is insufficient when the length of the computer run exceeds the $t$ ime allowed for a single job. The computer run of the model in Chapter 2 took 45 hours of computer $t$ ime, and the most allowed at one time on the MULTICS computer at MIT was 10 hours. As a result, the program had to be broken into several (8) pieces, and the job state, incliding all temporary results had to be saved at the end of processing each section. An additional problem is that in MACSYMA, the cost of saving and restoring the job state is enormous: it can even begin to contribute significantly to the computer time used. Therefore. the amount of information involved in the saving ard restoring processes must be minimized. This minimization requires a fair amount of finesse and care, but can be straightforward. For example, only the equations describing the left boundary, the lower-left corner and the upper left corner of the plasma domain need be in 
memory when the Jacobian calculation of the $x_{2}$-sweep on the left boundary is going on. That is, the equations for the mesh interior, the upper, $r i g h t$, and bottom boundary surfaces, and the upper-right and lower-right corners can all be left in storage during this section of the job run.

Other efforts can also make a positive contribution. One is to discard any intermediate result when it is no longer required for further processing. Again, care must be taken to analyze these situations carefully so as not to destroy a result before its importance has expired. A second strategy for minimizing what is in the job state is to defer the definition of variables and functias intil they are actually used. Finally, and perhaps most importantly, the infurmation lists used by the MACSYMA differentiation package, as well as those used by LISP functions we have added to the system, should be kept to a minimum. The storage used by these lists is not the issue; it is the time required by the functions using them to assess the applicability of each item on the list to every part of the expression being operated on. Two examples should clarify this discussion. First, DIFF uses a gradient and dependency database to determine whether the derivative of each atom in an expression will be zero or some other value. If it is known a priori that some of the dependency information in this database will have no influence on the differentiations performed in a section of the code, then that information should be removed from the database. In the case where the derivative of an "ATOM" (or subscripted variable, etc.) is zero, this removal is essential because DIFF must go through 
all that extra database information before it can conclude that the derivative is indeed zero. In the discretized version of the MHD equations, probably $50 \%$ of the "ATOM"'s have zero derivative when the Jacobian is being computed, so the time required to find the Jacobian can be significantly increased if the derivative database is not trimmed to the absolute minimum.

The second example deals with the database of constant quantities developed and used by the REDUCE_CONSTS function (this function will be discussed in Section 5.7). In this case, the current list of constants is examined to see if a given "ATOM" in the expression being operated on is a constant itself. If the list of constants contains a large number of constants which are known not to occur in the expressions in the current section of the computation, then just as with DIFF, the performance of REDUCE_CONSTS can be severely degraded.

\subsection{Fast Factoring and Optimizati n Functions}

So far, the discussion has emphasized the optimization of each aspect of the algebraic computation problem. But perhaps more critical is the need to generate optimal parallelizable code for use in a simulation code. This is due to the enormous amount of computer resources consumed by some simulation programs over their lifetime. For the purposes of optimization at this level, some of the techniques we will discuss also help in the algebraic transformation phase. For example, two important functions, GCFAC and REDUCE_CONSTS, can be 
used to keep the size of intermediate result expressions as small as possible. These functions serve the additional purpose here of minimizing the number of algebraic operations done in the innermost DO-loops of the generated FORTRAN where most of the speed gain from vectorization occurs. With judiclous application in concert with another function, GOPTIMIZE, they can be made to generate highly optimal code fragments of complex structure. Before fustifying this statement, a brief discussion of each of these functions is in order.

CCFAC is a general collection of factoring strategies whose major yoal is to make unwieldy expressions as compact and cheap to compute as possible. GCFAC therefore tends to restructure expressions the way physicists would do $1 t$ by hand. It is not completely successful because it makes no attempt to impose a predefined hierarchy on the expression. A new MACSYMA function. FACSUM, written by Richard Brenner of CalTech restructures expressions according to an imposed hierarchy, but does not do all the factoring that a physicist would like done. Coupled with GCFAC, FACSUM can do this in every case.

One of the criteria imposed early on in this work was that GCFAC would need to be fast. Indeed, early versions of GCFAC written in MACSYMA were so slow that it became obvious that this function alone could dominate the cost of code development. Many other expensive operations are necessary, so to make the project economically feasible, it was decided to rework the algorithm. One of the chief culprits for the slow behavior is the FACTOR function in MACSYMA. As this is at least in part due to the function being able to do multivariate 
factoring of multinomials, and l was inilially interested only in monomial-type factoring, GCFAC was rewritten in LISP with its own fast monomial factoring capability. A dramatic speedup of a factor of 200 was obtained in the rewrite and was due to two causes. First, there are substantial inefficiencies in running interpreted MACSYMA functions: translation into LISP has become an important operation for heavy MACSYMA users. By making the operations non-functional, i.e., by destroying the original operand in creating the result, more speedups were oblained. Also, at LISP level, there are speedups that can not be obtained at MACSYMA level. Second, by substituting a specialized factoring package for the MACSYMA FACTOR function, a much larger speed increase was obtained. Since FACSUM calls FACTOR to put coefficients into optimal form. it too suffers a speed degradation in most situations. This is because most general expressions which occur in physics applications cannot be factored at the outermost level of these expressions. Arriving at a practical multivariate. multinomial factoring package which is geared toward these applications and which does not suffer from the slow characteristics of FACTOR remains a research topic of considerable complexity.

A simple example illustrates the sort of factoring capability with GCFAC, which no other algebra function known to the author can perform:

(C2) $e x:(x-y) \cdot r+(x-y) \cdot s+z$; $Z+S(X-Y)+R(X-Y)$

(C3) $g c f a c(e x)$; 


$$
Z+(S+R)(X-Y)
$$

Here GCFAC detected that the expression $X-Y$ multiplied two terms in a mullivariate monomial, and that expression was factored out as illustrated on line (D3). GCFAC also performs other simple factoring over groups of terms. It factors integers out of these groups when possible. Also, it pulls out the largest factorable power of variables occurring as coefficients in the group of terms. In addition, some combinations of exponentials are factored. Finally, after all of these techniques have been exhausted, GCFAC attempts to factor pairs of terms, and steps down the terms in a sum to see if each adjacent pair will factor. All of these techniques are repeatedly and recursively applied until no further variation in the expression occurs.

The second structuring function REDUCE_CONSTS has no parallel el sewhere. It does not alter the basic hierarchy of the expression: rather, given a list of symbols which are known to be constanis, new constants replace products and quotients of constants. An optimal list of equations for these new constants is developed as the function evaluation proceeds, and each constructed constant is added to the constant list. If the density of constants in an expression is large, the reduction in overall expression size will be considerable. Contrariwise, REDUCE_CONSTS does not alter an expression containing no constant quantities or embedded products containing only one constant. Its operation is illustrated with the following simple example: 
(C1) ronst list:[ma, eps,q];

(D1)

$[M U: E P S, Q]$

(C2) $\varepsilon x: m u \cdot e p s \cdot t a u-q$;

(D2)

EPS MU TAU - Q

(C3) reduce_consts $(e x)$;

(Di)

$X X 1 T A U-Q$

(C4) const_list:

(DA)

$[M U, E P S, Q, X X 1]$

(C5) ronst eqns:

(D5)

$[X X 1=E P S M U]$

First of all, in line $(C t)$, the "ATOM"s $N U, E P S$, and $Q$ were declared to be constants in a way that makes that information available to REDUCE_CONSTS. The result of applying this function to an expression containing a product of those constants is lllustrated in lines (D3) through (D5) where the expression operated on is now more compact. In addition, the new, generated constant "ATOM", $X X 1$, has been added to the constant database, and its definition has been added to a list called CONST_EQNS. Using this latter information list. $R E D^{\prime \prime} C^{\prime} E_{-} C O N S T S$ can also determine if previously defined constants such as $X X Y$ occur in other parts of the same expression or in other expressions altogether. It does this by combining products of constants in an expression into new products which it then compares against the right-hand side of each of the equations in CONST EQNS. If one of the right-hand sides is equal to the new 
product, then the constant defined by that entry in CONSTEQNS replaces the new product in its parent expression.

The last optimization function mentioned in this section was COPTIMIZE. While it is an outgrowth of the MACSYMA OPTIMIZE function, additional provision is made for sequences of equations, not just single equations. Also, in a vectorizable setting, such as FORTRAN on the CRAY-1 provides, the temporary varlables assigned to common subexpressıons found by COPTIMIZE must be vector temporaries, not just scalars as is the case in the OPTIMIZE function. The kind of optimization done here is that typically done by most optimizing compilers: 1.e., a subexpression in an expression (or set of expressions) which occurs more than once is computed before the expressions in which it occurred. The result is assigned to a temporary which then takes the subexpression's place, wherever the subexpression occurred. The problem with this approach in a compiler is thit the analysis capability is usually limited because the unnatural environment of a compiler causes a large overhead in storage and processor time costs to perform that analysis. Moreover, the number of continuation lines available to an expression in all implementations of FORTRAN with which the author is familiar imposes an artificial breaking apart of any expression which exceeds the length restriction. This can make it impossible to find all common subexpressions, even if the compiler's overhead costs were not prohibitive. 
In algebra processing systems, especially those on virtual memory computers, no such restrictions need apply in practical cases (there are always examples one can contrive which will exceed the memory limitations of any computer). And for this reason, the efficient functionality of COPTIMIZE should be a real boon to the development of optimal code segments, wherever the target application might be. Because it breaks out common subexpressions from a sequence of equations, COPTIMIZE renders those equations of little further practical use in an algebraic environment, so it should be the last function applied to those equations or expressions before the FORTRAN function is applied to generate the actual code sequences. Note thai there is no real restriction here to any target language: one would simply replace the FORTRAN function with one which generated code for whatever other target language was of interest.

To illustrate exactly how COPTIMIZE works, consider the following simple example :

(C1) eqns: $[t=(u+v) \cdot y-c, s=x-(u+v) \cdot z / r]$

(D1)

$$
\left[T=(V+U) Y-C, S=X-\frac{(V+U) Z}{R}\right]
$$

(C2) goptimize(eqns);

$C$ - there are 2 occurrences of OPTtS

(D2) $\quad\left[O P T 1 S=V+U, T=\right.$ OPT1S $\left.Y-C, S=X-\frac{O P T 1 S Z}{R}\right]$

Here the label EQNS is bound to a list of two equations containing the obvious common subexpression $V+U$. By applying COPTIMIZE to that $1 \mathrm{ist}$, GOPTIMIZE informs 
us that it not orly found the common subexpression but informs us that it occurred twice in the list. The result is returned as a new list where the definition of the scalar temporary variable OPT1S is the first element of the list. Note also that OPT1S replaces $V+U$ wherever that expression occurred in the original equations.

We are now prepared to examine a more complex example where the use of GCFAC, REDUCE_CONSTS, and GOPTIMIZE are combined; only the FORTRAN output will be considered. First, consider the code generated by applying RATSIMP (a built-in MACSYMA function) to the expression for the fraction dissociated, $f_{d}$. and then using FORTRAN to generate the code. Since we want to compute this quantity for an array of values of the density, RHO, and ion temperature, TI. the $f$ inal FORTRAN segment looks like

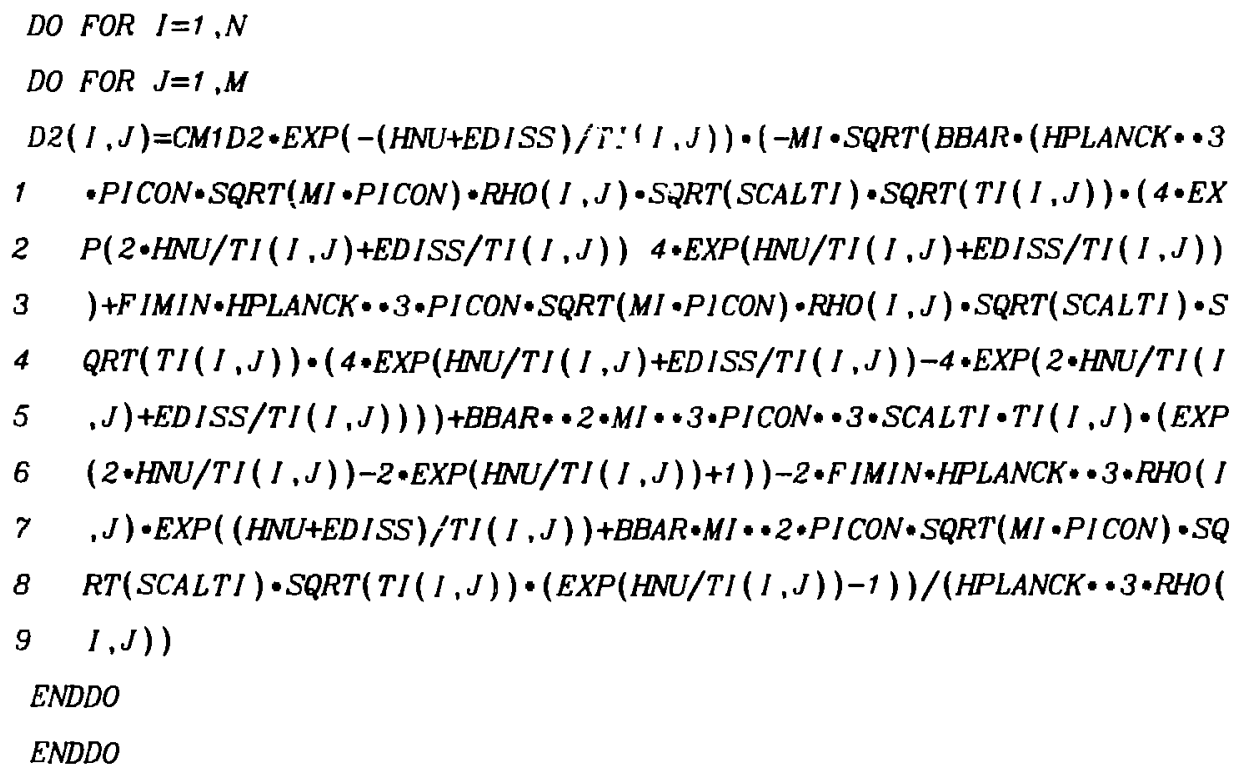


Here, CM1D2=-1/2; this and all other constant quantities are assumed to be initialized once and for all. If REDUCE_CONSTS, GCFAC, and GOPTIMIZE are now invoked before the FORTRAN function, a very different result is obtained:

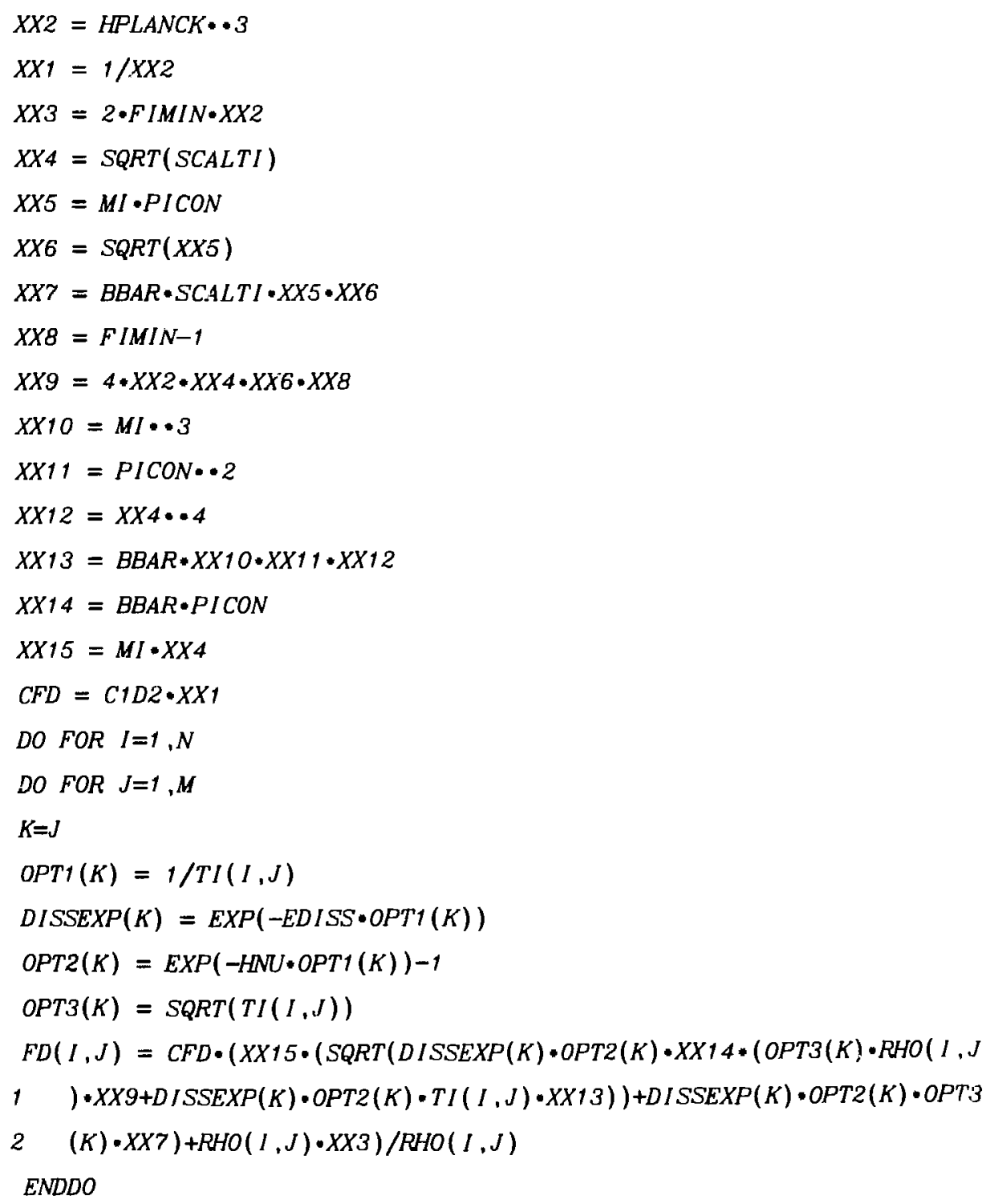


Observe that all computation performed for the constants (up to the line reading "CFD = $(1 D 2 * X X 1 ")$, is done only once. This alone leads to considerable speedup in the computation of the DO-lorp. But then the considerable compactification done by GCFAC cuts down the size of the expression by a great deal more. There isn't much left to optimize, but still. GOPTIMIZE finds some new as well as predetermined common subexpressions, which further enhances the DO-loop's efficiency.

An interesting note in passing is that while the two code segments are indeed algebraically equivalent. the second is more numerically stable. This is due in all probability to two phenomena. First, large amounts of cancellation can occur when a difference between two nearly identical quantities is computed, and there are several instances in the first DO-loop where this could occur. Notably, only OPT2(K) in the second DO-loop is a candiate for such an effect. The second numerical instability phenomenon is that which arises from the gradual but steady accumulation of roundorf errors. This effect is most likely to occur from the sheer volume of operations performed, which again makes the first Do-loop a more likely candidate for numerical instability. 


\subsection{Use of Large Virtual Memory Computers}

The last point in this chapter comes as a surprise in view of all of the work of the previous sections discussing means of absolutely minimizing the the use of computer memory and processing time. The unfortunate fact of the matter is that for large physics problems, a large virtual memory computer with the algebraic language of interest available on it is an unavoidable necessity. In other words, the scale of problem is still the dominating factor, and the fact that the scale of the MHD equations in Chapter 2 is simply enormous means that only a very large virtual memory computer system is capable of handling the algebra involved. This scaling problem was substantiated by experience as that problem expanded to consume about 15,000,000 words of memory at one point during the computation on the MULTICS computer at MIT. Curiously, at the time of this writing, the MULTICS computer was the only one available with that amount of memory which ran one of the major computer algebra programs.

For smaller problems, of course, this amount of computer power is not essential. Therefore, very interesting sets of PDEs could be processed on computers such as the VAX and the LISP MACHINE. In conjunction with this scale down in the size of the physical model, it is also interesting to observe that only modest size models can be processed on memory-limiled computer systems. This point is substantiated in the next chapter where the examples were done on the MIT-MC DEC-10 computer. 


\section{CHAPTER 6}

\section{HOW PDES ARE TRANSFORMED INTO FORTRAN}

\subsection{Introduction}

Chapter 5 unveiled the tools and strategies to allow a formidable set of PDEs to be transformed into FORTRAN code segments. But even with these tools, the actual achievement of code generation came only with several thousand more lines of MACSYMA and LISP programing. This code generation is not discussed in detail: only the most important aspects will be explicitly illustrated. The discretization techniques to be used in the two examples are those of Chapters 3 and 4 . 


\subsection{Example 1}

The first example is so simple that the concepts and flow of the processing are clear. The model is composed of the single partial differential equat ion

$$
\frac{\partial P}{\partial t}=-\nabla \cdot(\gamma P \mathbf{v})+(\gamma-1) \mathbf{v} \cdot \nabla P .
$$

This contrived one-equation system represents the time development of a fluid pressure, $P$. $\vee$ is assumed to be a known flow field, and $\gamma$ is the ratio of specific heats of the gas or fluid.

\subsubsection{MACSYMA Initialization}

Since there is only one dependent variable, $P$, the global NEQS is set to $1:$

(C3) neqs:1\$

In conjunction with this, the global DEPVARS is set to the list containing only $P:$

(C4) depvars:[P];

(D4)

The components of the external flow field are defined by the next MACSHMA IIHes: 
(15) $1:[1,1,22,23]$

(Dis)

$\left[v 1, v^{\prime}, v 3\right]$

Declaring the formal dependencies of all variables is the object of the following code:

(C6) depends $([v 1, v 2, v 3, P],[r, t h])$ :

(D6)

$[V(R, T H), V 2(R, T H), V 3(R, T H), P(R, T H)]$

Assuming that the natural coordinate system describing the probitii $1 \leq$ a polat toroidal one, those coordinates are chosen by assip".ing COORDINATES to a description of the generalized coordinates and H_FACTORS to the scale factors of polar toroidal coordinates:

(C7) coordinates: $\left[x^{1}=r, x 2=t h, x 3=p h i\right]$;

(D7)

$$
\left[X_{1}=R, X 2=T H, X 3=P H I\right]
$$

(c8) $h$ factors $:[h 1=t, h 2=r, h 3=R O+r * \cos (t h)]$;

(DB)

$$
[H 1=1, H 2=R, H 3=R \operatorname{COS}(T H)+R O]
$$




\subsubsection{Discretization of the PDE}

The first ad hoc measure is to assume that discretization of the time derivative is built into the underlying MACSYMA and LISP functions. While functionality to perform this discretization automaticaliy was developed for the integro-differential equations describing the behavior of the external coils, it

was not done for the MHD equations, hence the ad hoc assumption. With this in mind, the global label SRHS is assigned to the right-hand side of the pressure equation.

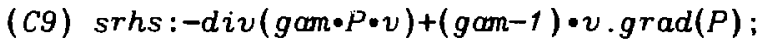

(D9)

$-\frac{\frac{d}{d X 3}(G A M H 1 H 2 P V 3)+\frac{d}{d X 2}(G A M H 1 H 3 P V 2)+\frac{d}{d X 1}(\text { GAM H2 H3PV1) }}{H 1 H 2 H 3}$

$+\frac{(G A M-1) \frac{d P}{d X 3} V 3 \quad(G A M-1) \frac{d P}{d X 2} V 2 \quad(G A M-1) \frac{d P}{d X 1} V 1}{H 2}$

Next, a function called PDE_LEVEL_GRADIENTS is called. This function performs a variety of operations. Among them are establishing gradient information for use in the expansion of the PDEs, defining the names to be used for derivatives of the dependent variables on the boundary as well as at interior mesh points, and setting up the related spline derivative coefficient information according to the description in Chapter 3 and Appendix B. Some of 
the results of calling this function can be observed by having MACSYMA print out the gradient information which it established.

$$
\begin{aligned}
& \text { (C13) printprops(all, atomgrad)\$ } \\
& \frac{d P d r m(K)}{d P K(i-1, j)}=S 11 M D V K M(I) \\
& \frac{d \operatorname{Pdrm}(K)}{d P K(I, J)}=S 11 M \operatorname{MVK}(I) \\
& \frac{d P d r m(K)}{d P(i-1, j)}=S 11 M D M(I) \\
& \frac{d P d r m(K)}{d P(I, J)}=\operatorname{S1MDV}(I) \\
& \frac{d P d r p(K)}{d P K(i+1, j)}=S 11 P D V K P(I) \\
& \text { (29 other gradient definitions) }
\end{aligned}
$$

As mentioned in the last chapter, PROCESS_PDE_STRUCTURE is the key function which transforms the PDEs into convective form. The MACSYMA environment is now prepared to do just that. But first, a simpler example of how this function works should be given.

(C1) neqs: $1 \$$

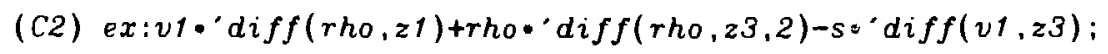




$$
-S \frac{d V 1}{d Z 3}+\frac{d R H O}{d Z 1} V 1+R H O \frac{d^{2} R H O}{d Z 3}
$$

(C3) l:process_pde_structure $([[1, e x]])$;

$$
\left[V 1,0, R H O \frac{d^{2} R H O}{d Z 3},-s \frac{d V 1}{d Z 3}\right]
$$

As in the pressure PDE, only one dependent variable is in the system, and by default it is $R H O$. Also, the independent variables have been defaulted to $Z 1$ and $Z 3$ instead of $X 1$ and $X 3$. Of particular importance in this example is that a structured version of EX is passed to PROCESS_PDE_STRUCTURE, not just EX. As was mentioned in the last chapter, this structuring process is done by hand: it has not been automated. The interpretation of the components of the result on line (D3) are as follows: the first NEQS components are the coefficients of the first derivatives with respect to $Z 1$ of the dependent variables (which are NEQS in number). Similarly, the next NEQS components are the coefficients of the first derivatives with respect to $Z 3$. The next component contains all terms in the PDE which invclve second derivatives (not mixed). And the final component contains all explicit terms, including any mixed second derivatives.

As this strange output form requires that any function operating on $\mathrm{i}: \mathrm{s}$ output understand the above description, the function which handles the next stage will now be described. It is TOTAL_COLLAPSE. And as its name suggests, TOTAL_COLLAPSE collapses all of the derivative forms contained in the $1 \mathrm{is}$ : 
passed to it according the prescription of Chapter 3 . The following example (again, not related to the pressure PDE) illustrates what TOTAL_COILAPSE does to the output of PROCESS_PDE_STRUCTURE ( $L$ in this case).

(C4) total_collapse $(1,[])$ :

$C$ - lector values needed for: $\operatorname{V1dZ3(K)}$

$(D 4)-S V 1 d Z 3(K)+C V M G P(R O D Z 1 M(K), \operatorname{RODZ1P}(K), V 1(I, J)) V 1(I . J)$

$+\operatorname{PRHO}(1, J) \operatorname{ROD} 2 Z 3(K)$

A single expression is returned. Note that there is a second argument to this function, which is an empty list in this case. The usual entries expected in this list are derivatives which are to be set to zero. This list of derivatives is useful, for example, if, in processing equations at the boundary, certain derivatives are known to be zero. A case in point is the derivative of either temperature variable (electron or ion) in the direction normal to the boundary. This derivative is zero according to the insulating wall condition discussed in Chapter 2 .

Also of interest in the above example is the message which TOTAL_COLLAPSE prints concerning the FORTRAN vector " $V 1 d Z 3(K)$ ". This information is given to let us know that there was no information in the gradient database for $\partial_{23} V 1$, the spatial derivative of an explicit quantity, and that some means must be provided to compute it. 
A second interesting point is that $R H O$ has been replaced by "PRHO $(I, J)$ " instead of "RHO $(I, J) "$ as the coefficient of "ROD2Z3(K)". PRHO is the array $R H O$ with any negative values replaced by a "floor" positive value. This guaranteed positive value multiplies the discretized value of the second derivative of $R H O$ with respect to 23 for numerical stability reasons discussed in Chapter 3 .

There is also the appearance of the obscure form, CVMCP. It is a CRAY-1 intrinsic function which returns its first argument if the third argument is greater than or equal to zero, and returns the second argument otherwise. Through this sign detection mechanism, the upwind evaluation of the main first derivative terms in the convective form of the MHD equations can be implemented as described in Chapter 3.

With these two major functions, PROCESS_PDE_STRUCTURE and TOTAL_COLLAPSE, the model PDEs are prepared for the Jacobian calculation and the setting up all other expressions required by the solver presented in Chapter 4 . So we do this preparation for the pressure equation with the following block of MACSYMA code 


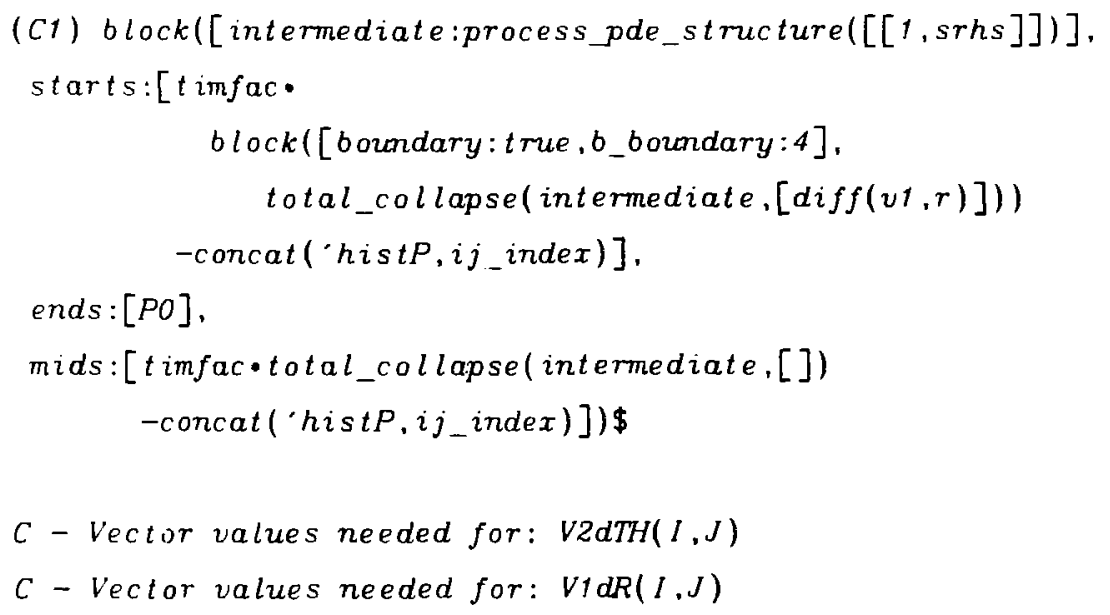

In this sample, STARTS represents the equation which applies at the bottom boundary of the pressure PDE's domain, while MIDS represents the equation in the interior of that domain, and ENDS represents the equation at the top boundary. The fact that the collapsed results are multiplied by TIMFAC and that a history-type constructed variable is subtracted from the result is simply a mnemonic device to represent part of the effect of performing the time discretization of the PDE. While on the bottom boundary, note that TOTAL_COLLAPSE is required to zero out the derivative of $V 1$ with respect to $R$. This would represent a slip boundary condition on the velocity field of the fluid. Also, both the setting of the global BOUNDARY to TRUE and B_BOUNDARY to 4 are essential if the system is to understand that processing is occurring at the bottom boundary. 
At the top boundary, a time-independent boundary condition is imposed. This is accomplished by requiring $P$ be set to $P O$ without any calls to PROCESS_PDE_STRUCTURE or TOTAL_COLLAPSE.

As in the illustration of the operation of TOTAL_COLLAPSE above, that function informs us that there were two derivatives of explicit variables for which no gradient information was provided. So the system generates appropriate array names for them, "V2dTH(I,J)" and "VIdR(I,J)", and tells us that their values must be computed elsewhere. Other important results of this MACSMMA block are the final values of MIDS and STARTS. First of all, MIDS will be displayed:

$$
\begin{aligned}
& \text { (C2) mids; } \\
& \text { (D2) [TIMFAC (- GAMP }(I, J)((R(I) \operatorname{COS}(T H(J))+R O) \quad V 2 d T H(I, J) \\
& -R(I) \sin (T H(J)) V 2(I, J)+\left(R(I)^{2} \cos (T H(J))+R(I) R O\right) V I d R(I, J) \\
& +(2 R(I) \cos (T H(J))+R O) V I(I, J)) /(R(I)(R(I) \cos (T H(J))+R O)) \\
& -\frac{\operatorname{CVMCP}(P d t h m(K) \cdot \operatorname{Pd} t h p(K),-V 2(I, J)) \operatorname{V2}(I, J)}{R(I)} \\
& -\operatorname{CVMCP}(\operatorname{Pdrm}(K), \operatorname{Pdrp}(K),-V 1(I, J)) V 1(I, J))-\operatorname{HISTP}(I, J)]
\end{aligned}
$$

Observe that there are no derivative forms remaining in this list and that there are two upwind forms in the expression, computed via CVMCP for numerical stabilization of the convective derivatives. Finally, consider STARTS: 


$$
\begin{aligned}
& \text { (C:3) starts: } \\
& (D 3)[\operatorname{TIMFAC}(-\operatorname{CAM} P(I, J)((R(I) \operatorname{COS}(T H(J))+R O) \operatorname{V2dTH}(I, J) \\
& -R(I) \operatorname{SIN}(T H(J)) V 2(I, J)+(2 R(I) \operatorname{COS}(T H(J))+R O) V I(I, J)) \\
& /(R(I)(R(I) \operatorname{COS}(T H(J))+R O))-\frac{\operatorname{Pdthp}(K) V 2(I, J)}{R(I)} \\
& -\operatorname{ClMCP}(\operatorname{Pdrm}(K), \operatorname{Pdrp}(K),-V t(I, J)) V t(I, J))-\operatorname{HISTP}(I, J)]
\end{aligned}
$$

This expression is essentially the same as MIDS with one major difference: one of the upwind forms is no longer there. Indeed, given that the $X 2$ direction is $T H$. the upwind evaluation of $\partial_{T H} P$ at the bottom boundary would require that a value of $P$ be available outside the domain of the PDE. This is disallowed, so upwinding is not done at the boundaries: first derivative evaluation always orrurs towards the domain interior at a boundary.

\subsubsection{Jacobian Calculation and Code Generation}

Everything is now in readiness to $f$ ind the Jacobian and generate the required code segments. However, to understand the short code block which 1)lustrates this final step, it will be necessary to understand several more functions. They are: DO_PREOPTIMIZATION, JACOBIAN_MATRIX, X_SWEEP_RHS. GENRATE_CODE_BLOCKS, GENERATE_DECLARATIONS, OPEN_FORTRAN_FILE, and CLOSE_FORTRAN_FILE. 
First, DO_PREOPTIMIZATION is a function which krows about the parts of each expression which will not be involved in the Jacobian calculation and "optimizes" them away in a sense similar to that done by GOPTIMIZE.

JACOBIAN_MATRIX performs the mammoth task of computing the elements of the Jacobian matrix. Consequently, the majority of the processing time will be spent in this function, and any improvements in its efficiency will yield handsome rewards. The first argument of JACOBIAN_MATRIX is the matrix whose elements are to be found, these matrices arising from the matrix splitting scheme discussed in Chapter 4. If a different solution technique were to be implemented, then an entirely ncw function would be required, because JACOBIAN_MATRIX takes full account of the sparsity and structure of these matrices. The second argument to this function simply provides the formal subscript or mesh index which corresponds to the current matrix designated by the first argument. A third and final argument carries some arcane vectorization and indexing information of significance only in the FORTRAN output.

Once the matrices involved in the solution process are formally complete, it still remains to $f$ ind the vector of algebraic expressions making up the right-hand side vector. This assembly is done by $X_{-} S W E E P \_R H S$ for the solve coming from the first direction or $X 1$ splitting. GENERATE_CODE_BLOCKS then uses this vector and the matrices found by JACOBIAN_MATRIX to assemble the various code blocks which will be generated and optimizes these code blocks. Then it 
uses OPEN_FORTRAN_FILE and CLOSE_FORTRAN_FILE (discussed below) to put the FORTRAN versions of those code blocks into a series of different files, each file containing the FORTRAN counterpart of only one code block. This modularization of the code pieces is intended to make the editing and assembling of the final code a much easier task.

Little remains to be done after GENRATE_CODE_BLOCKS is finished. Nonetheless, the information gathered during the course of the algebraic computation can now be employed to great advantage by using it to lay out the COMMON areas, dimension all of the temporary and "permanent" vectors and arrays, initialize the COMMON areas containing derived and intrinsic constants, set up pointer information, and initialize a menory-management package (in FORTRAN) for the main vectors and arrays. GENERATE_DECLARATIONS does all of this using the database which has been built up.

The last two functions, OPEN_FORTRAN_FILE and CLOSE_FORTRAN_FILE, act in concert to open and then close an output file into which the FORTRAN function writes code. When this file is opened, all FORTRAN output is directed to the file as well as to the computer terminal. Moreover, no other terminal input or output gets into the file, thus preserving it as a purely FORTRAN output file. The name of the file is the single argument to OPEN_FORTRAN_FILE, while CLOSE_FORTRAN_FILE is a function of no arguments. 
With these explanations, the following code segment should be a little more transparent.

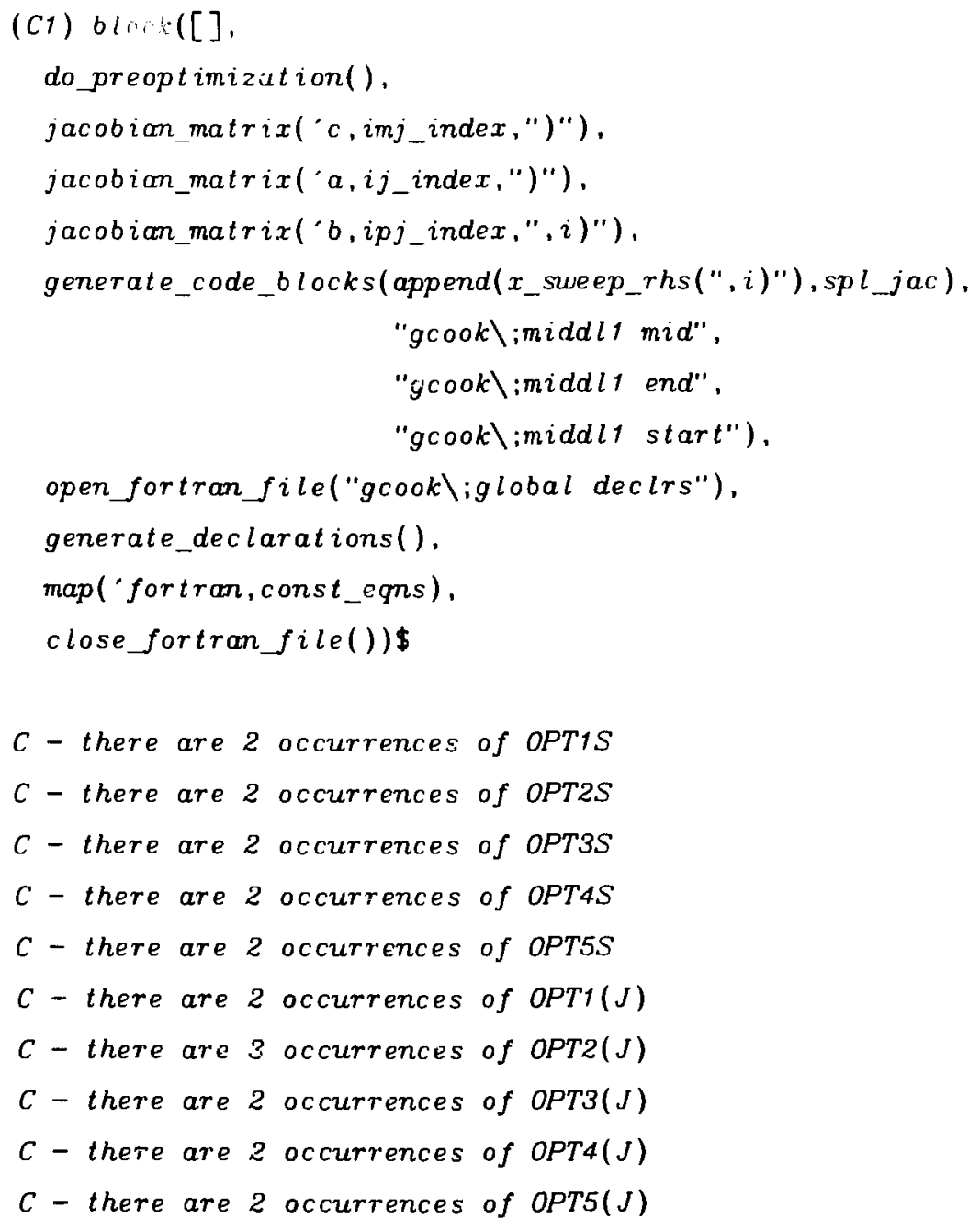

Observe that two kinds of code segments have been generated in this segment by the call to GENERATE_CODE_BLOCKS. The evidence for this is seen in the fact that both scalar and vector temporary optimized variables occur in the code 
blocks. The exact significance of these temporaries can be seen in the FORTRAN output for these code blockss seen below. The three calls to JACOBIAN_MATRIX are not sufficient to define all of the matrices involved in the matrix splitting scheme. In fact, five more segments of MACSYMA code like the one above are required to complete the definitions. They are, however, very similar to the one given here, and so this example will suffice.

A few variables in this example also bear some explanation. The second argument to JACOBIAN_MATRIX is expected to be an index; the particular indices being passed here are

$$
\begin{aligned}
& I M J_{-} I N D E X="(I-1, J) " \\
& I J_{-} I N D E X="(I, J) " \\
& I P J_{-} I N D E X="(I+1, J) "
\end{aligned}
$$

In the first argument to GENERATE_CODE_BLOCKS is found the variable SPL_JAC It is a list of contributions to the right-hand side vector in the solver from the spline tridiagonal relations. Finally, the variable CONSTEEQS was seen in the last chapter. There, it was defined as a list of equivalences defining constructed constants, and therefore fits into the domain of the generated internal database. In the above segment, the FORTRAN function is mapped over this list at such a point that these equations will appear after the memory management system has been initialized, at the very beginning of the executable code. 
There are four separate output files of FORTRAN which were created by executing the above block of MACSYMA. It is instructive to examine their contents. First, consider the file "gcookimiddll mid":

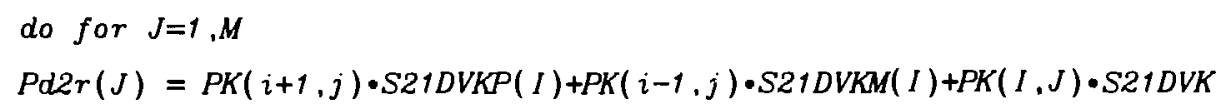




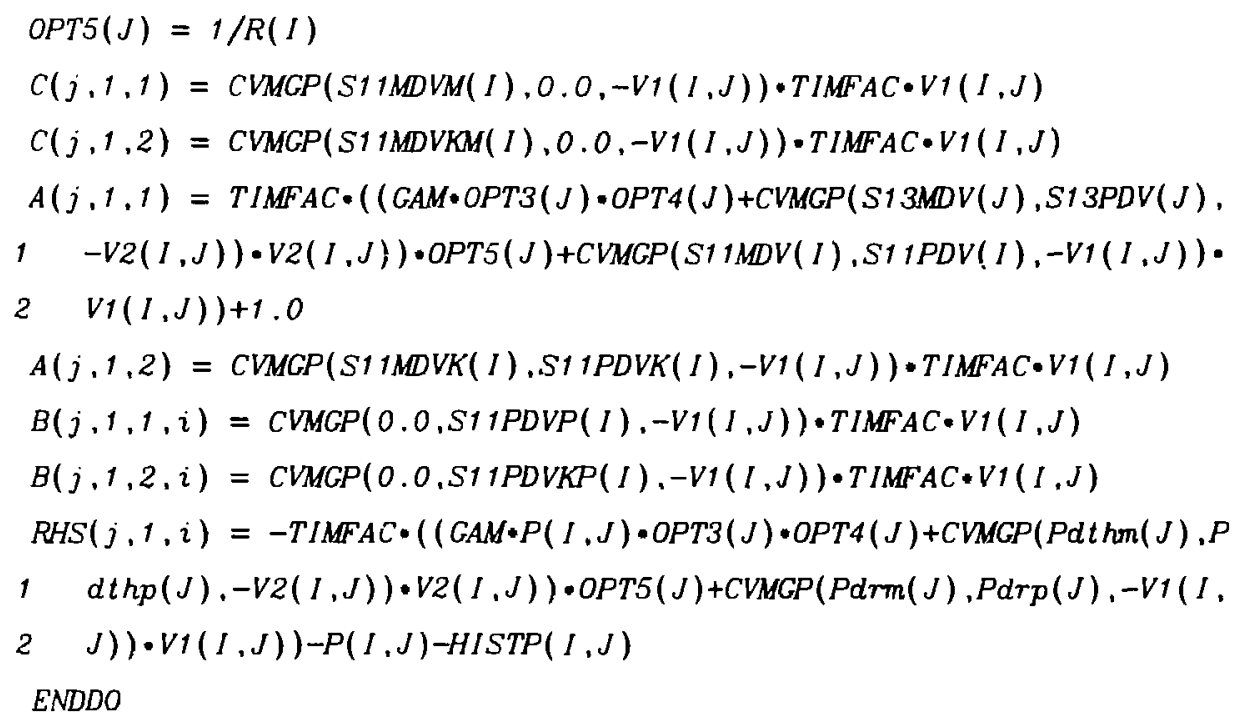

In this section of code there are two DO-loops which are not standard FORTRAN. Indeed, the form

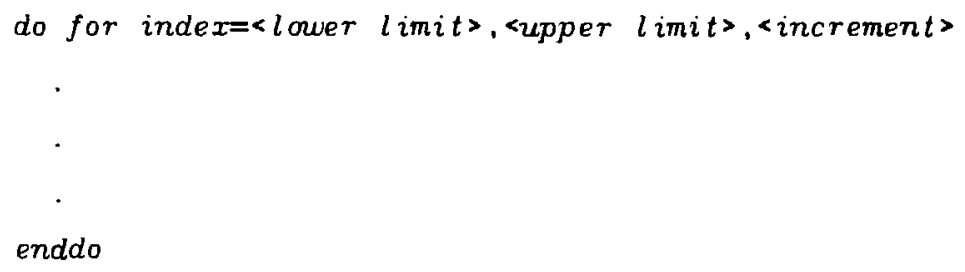


At the beginning of each DO-loop, several one-sided first derivative approximations are computed. The first approximations are the derivatives of $P$ with respect to $R$ and those in the second DO-loop are taken with respect to $T H$. In the first loop again, the array RHS corresponds to the vector of right-hand side values required by the solution technique of Chapter 4, while the $C, A$, and $B$ arrays contain Jacobian elements. Other matrix elements are also found in the second loop. The most interesting part of the second loop, however, is the part where five temporary vectors are computed. These temporaries are then used in later expressions.

Next, consider the file "gcook;middll end":

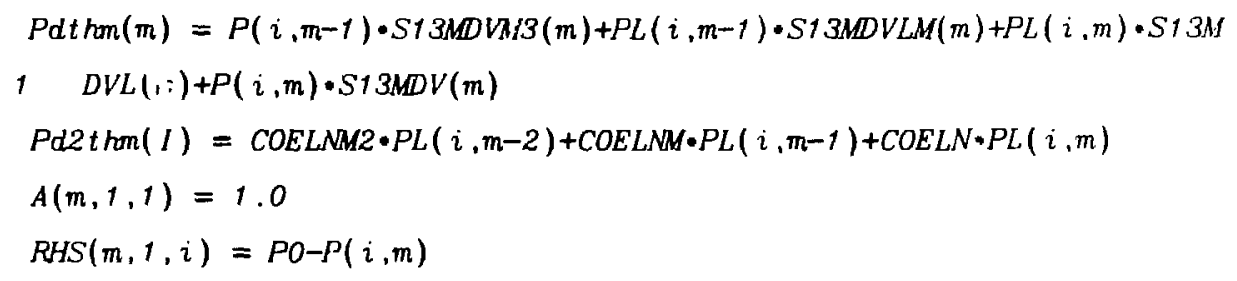

The contents of this segment are extremely simple, owing to the time-independent boundary condition which was imposed. Short as it is, however, the first two statements are superfluous, a fact that GENERATE_CODE_BLOCKS is not smart enough to ascertain.

Code for the other boundary is found in the file "gcook;middll start". and a listing follows: 


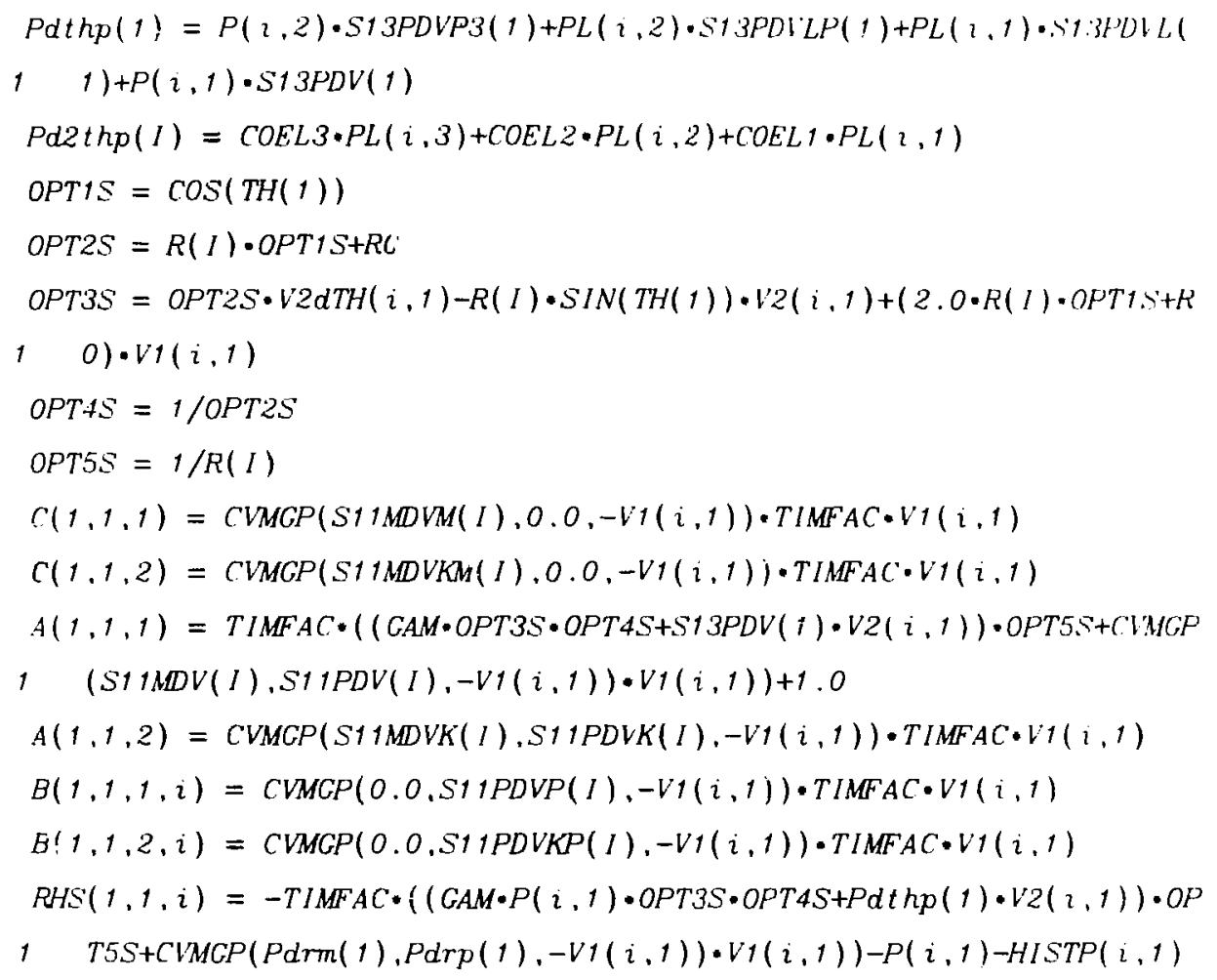

Observe that the code here is similar to that in the first FORTRAN code block. However, there are no DO-loops. This means that all of the optimized temporary varlables are scalars instead of vectors, and that the code will not run as fast although it is still "optimal" in the scalar sense.

The last file is "gcookiglobal declrs". As its name suggests, it contains global and declarative-type FORTRAN statements:

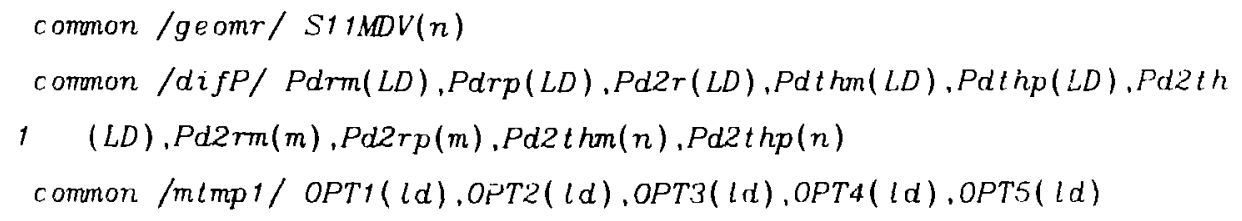




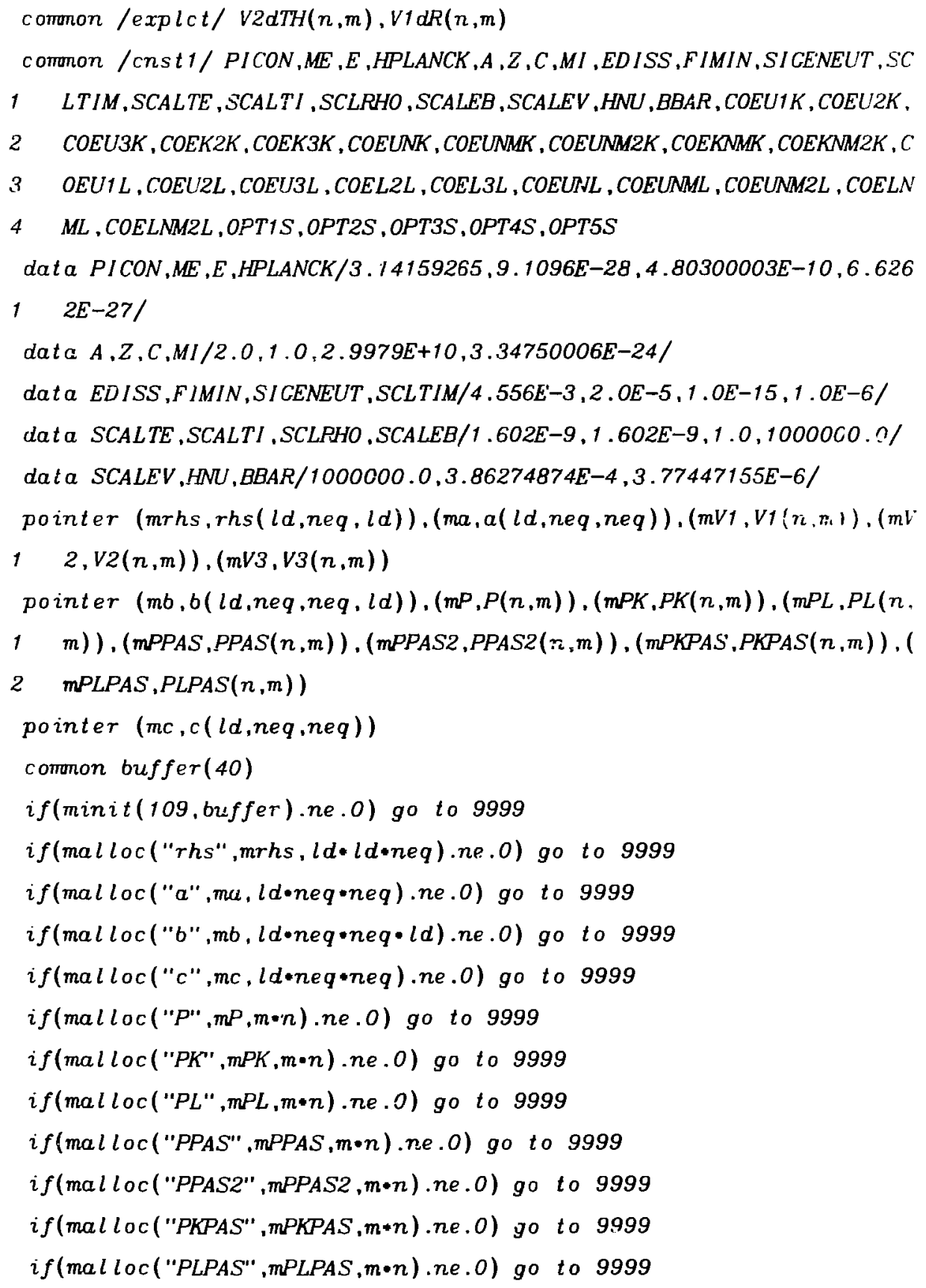

The POINTER statements are new FORTRAN declarations which set up base address 
information for dynamic array management applications. Among the arrays and vectors we treat in this manner are the Jacobian matrices, the right-hand side vector, and the mesh values of the principal physics variables at each required time level. In the executable code section. the functions MINIT and MALLOC are part of the A-division memory management package used at LLNL. Details of their operation will not be given here.

\subsection{Example 2}

The second major example of this chapter is a simple MHD model. Although the model is simple, the PDEs are not, and this is reflected in the substantially increased difficulty in performing even a fraction of the algebra on the MIT-MC computer. The model begins with a mass cont inuity equation

$$
\frac{\partial \rho}{\partial t}=-\nabla \cdot(\rho \mathbf{v})
$$

Tt lere are the momenta equations:

$$
\frac{\partial \mathbf{v}}{\partial t}=\frac{\ddot{\rho}}{\rho}\left[-(\boldsymbol{v} \cdot \nabla) \mathrm{y}-\nabla P+\frac{\boldsymbol{J} \times \boldsymbol{\theta}}{c}\right] .
$$

As this is a single temperature model, there is only one energy equation: 


$$
\frac{\partial T}{\partial t}=\frac{1}{\rho C_{\nu}}[-P \nabla \cdot v+\nabla \cdot(\kappa \nabla T)]
$$

Faraday's law is assumed to have the very simple form

$$
\frac{\partial \boldsymbol{B}}{\partial t}=-\nabla \vee(\mathbf{v} \times \mathbf{B})
$$

with no external component to the $B-f$ ield. The system is closed by an adiabatic equation of state

$$
P=C_{V}(\gamma-1) \rho T
$$

and by equation (2.110), a simplified version of Ampere's law. In these equations, $C_{V}$ is the specific heat of the plasma, $\kappa$ is the heat conductivity (here assumed to be a constant), and $\gamma$ is the ratio of specific heats.

\subsubsection{MACSYMA Initialization}

As before, the algebraic computation begins with some initialization steps. First, note that several quantities are constants in the above physical model. For example, $c, \gamma(G A M)$, and $C_{V}$ (CSUBV) are constants, and, along with some scaling constarts, are used to establish the initial constant database:

(C4) CONST_LIST:[PICON,C,SCALT, SCLRHO, SCALEB, SCALEV ,GAM, CSUBV];

(D4) [PICON, C, SCALT, SCLRHO, SCALEB, SCALEV, GAM, CSUBV] 
In order to have these quantities initialized in DATA statements by GENERATE_DECLARATIONS, DATAVARS and DATAVALS must be set to the appropriate values. Lines (C5) through (D6) accomplish this

(C5) DATAVARS:[PICON,C,SCLTIM, SCALT, SCLRHO , SCALEB, SCALEV, GAM, CSUBV];

(D5) [PICON, C, SCLTIM, SCALT, SCLRHO, SCALEB, SCALEV, GAM, CSUBV]

(C6) DATAVALS:[EV(\%PI,NUMER), 2.9979E10,1.E-6,

$$
1.602 E-9,1 \ldots 1 . E 6,1 . E 6,5 . / 3 ., 2.1] \text {; }
$$

(D6) $[3.14159265,2.9979 E+10,1.0 E-6,1.602 E-9,1.0,1000000.0$.

$1000000.0,1.66666667,2.1]$

NEQS, the number of dependent variables, and DEPVARS, the list of the names of those dependent variables, are set up next:

(C7) $N E Q S: 8 \$$

(C8) DEPVARS: $[R H O, V 1, V 2, V 3, T, B 1, B 2, B 3]$;

$[R H O, V 1, V 2, V 3, T, B 1, B 2, B 3]$

Next, define the components of the velocity and $B-f$ ield vectors:

(C9) $V:[V 1, V 2, V 3]$;

(D9)

$[V 1, V 2, V 3]$

(C10) $B:[B 1, B 2, B 3]$;

(D10)

$[B 1, B 2, B 3]$ 
Now, establish the formal dependencies of the dependent variables:

(C11) DEPENDS([RHO,V1,V2,V3, ],B1,B2,B3],[R,Z]);

(D11) $[R H O(R, Z), V 1(R, Z), V 2(R, Z), V 3(R, Z), T(R, Z), B 1(R, Z)$,

$$
B 2(R, Z), B 3(R, Z)]
$$

In this example, the coordinate system is chosen to be cylindrical coordinates. MACSYMA lines (C1Z) through (D13) establish the variables COORDINATES and H_FACTORS in accordance with this choice.

(C12) COORDINATES: $[X 1=R, X 2=T H, X 3=Z]$;

(D12)

$$
[X 1=R, X 2=T H, X 3=Z]
$$

(C13) H_FACTORS: $[H 1=1, H 2=R, H 3=1]$;

(D13)

$$
[H 1=1, H 2=R, H 3=1]
$$

Bindings are now effected for the pressure and current density variables:

(C14) P:REDUCE_CONSTS $((G A M-1) \cdot C S U B V \cdot R H O \cdot T)$ :

(D14)

$X X 2$ RHO T

(C15) $J: R E D U C E \_C O N S T S(C /(4 \cdot P I C O N) \cdot C U R L(B))$; 


$$
\begin{aligned}
& \text { (D15) }\left[-\frac{D 4}{D X 2}\left(\frac{D}{\left.D 3 H 3)-\frac{D}{D X 3}(B 2 H 2)\right)}\right)\right. \\
& \text { XX4 } \left.\frac{D}{D X 3}(B 1 H 1)-\frac{D}{D X 1}(B 3 H 3)\right) \quad X X 4\left(\frac{D}{D X 1}(B 2 H 2)-\frac{D}{D X 2}(B 1 H 1)\right)
\end{aligned}
$$

Of particular note in these definitions is the fact that some sums and products of constants have been replaced by the new constants $X X Z$ and $X X 4$. Their definitions can be found in the executable code section of Appendix $E$. The resulting expressions in each case are more compact than they would otherwise have been, a result that has significant beneficial consequences when intermediate expression swell occurs later in the calculation.

For convenience in working with the vector equations. BRHS is set to the right-hand side of equation (6.5) and VRHS to the right-hand side of equation $(6.3):$

(C16) BRHS:-CURL $(\operatorname{CROSS}(V, B))$;

$$
\begin{aligned}
& (D 16)\left[-\frac{\frac{D}{D X 2}(H 3(B 2 V 1-B 1 V 2))-\frac{D}{D X 3}(H 2(B 1 V 3-B 3 v 1))}{H 2 H 3},\right. \\
& -\frac{D}{D X 3}(H 1(B 3 V 2-B 2 V 3))-\frac{D}{D X 1}(H 3(B 2 V 1-B 1 V 2)) \\
& -\frac{D X 1}{D X 1}(H 2(B 1 V 3-B 3 V 1))-\frac{D}{D X 2}(H 1(B 3 V 2-B 2 V 3))
\end{aligned}
$$


(C17) VRHS: $\left(-V \_D O T D E L O P \_V(V, V)-G R A D(P)+C R O S S(J, B) / C\right) / R H O$;

$(D 17)\left[\left(\left(-B 3 \times X 4 \frac{D}{D X 3}(B 1 H 1)-\frac{D}{D X 1}(B 3 H 3)\right)\right.\right.$

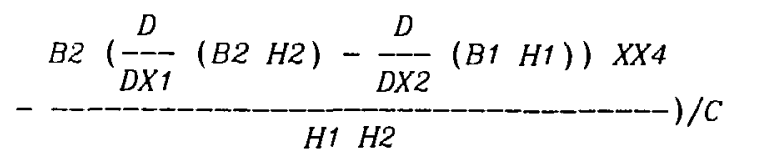

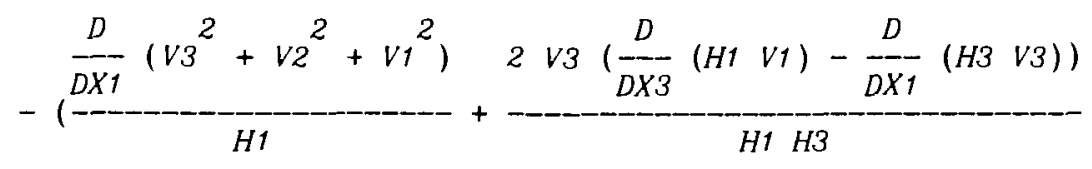

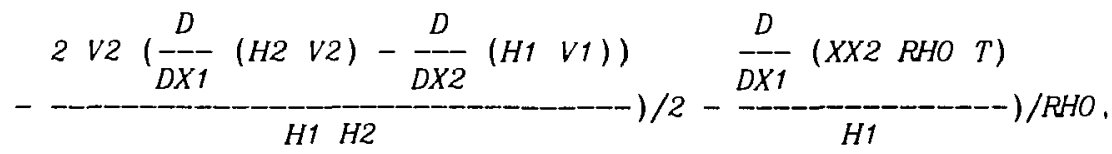

$(1)^{B 1} \times X 4\left(\frac{D}{D X 1}(B 2 H 2)-\frac{D}{D X 2}(B 1 H 1)\right)$

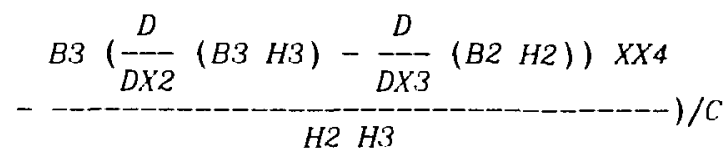

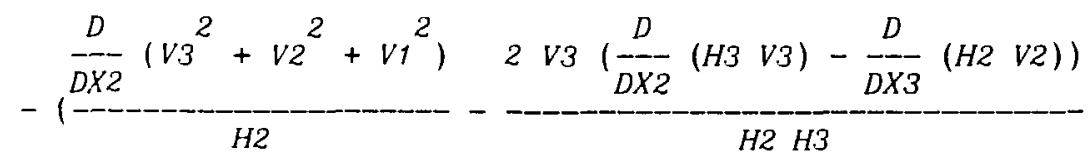

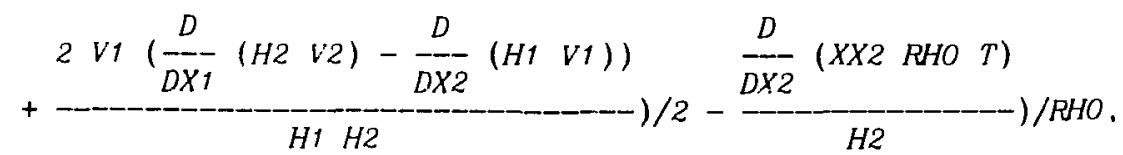

$B 2 X X 4\left(\frac{D}{D X 2}(B .3 H .3)-\frac{D}{D X .3}(B 2 H 2)\right)$

H2 H.3 


$$
\begin{aligned}
& B 1\left(\frac{D}{D X 3}(B 1 H 1)-\frac{D}{D X 1}(B 3 H 3)\right) \times X 4 \\
& \text { H1 } H 3 \\
& \frac{\frac{D}{D \times 3}\left(v 3^{2}+v 2^{2}+v^{2}\right)}{H 3}=\frac{\left(v 2 \frac{D}{D \times 2}(H 3 v 3)-\frac{D}{D \times 3}(H 2 v 2)\right)}{H 2 H 3}
\end{aligned}
$$

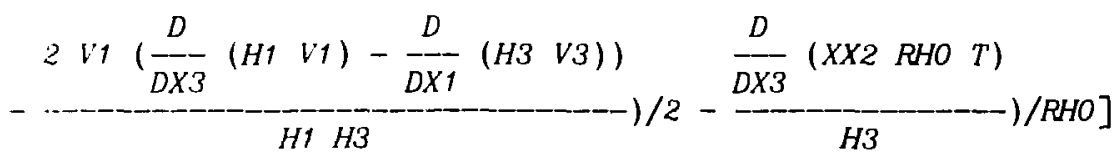

While the vector notation for VRHS is fairly compact, the corresponding generalized orthogonal coordinate representation is already huge. And while some derivatives will evaluate to zero because the equations are assumed to depend on only two space variables, application of the derivative operators will cause a large increase in the size of the expressions.

\subsubsection{Discretization of the PDEs}

For the sake of convenience in processing the MHD model, the $\mathrm{r}$ ight-hand sides of equations (6.2) through (6.5) are assembled into the vector $F$.

(C18) $F[1]:-D I V(R H O \cdot V)$;

$$
\begin{aligned}
& \frac{D}{D X 3}(H 1 H 2 R H O V 3)+\frac{D}{D X 2}(H 1 H 3 R H O V 2)+\frac{D}{D X 1}(H 2 H 3 R H O V 1) \\
& (D 18)-\frac{H 1 H 2 H 3}{(C 19) F[2]: \operatorname{VRHS}[1] \$} \\
& \text { (C20) } F[3]: \operatorname{VRHS}[2] \$ \\
& \text { (C21) } F[4]: \operatorname{VRHS}[3] \$
\end{aligned}
$$


(C22) $F[5]:(-P \cdot D I V(V)+D I V(K A P P A \cdot C R A D(T))) /(R H O \cdot C S U B V)$;

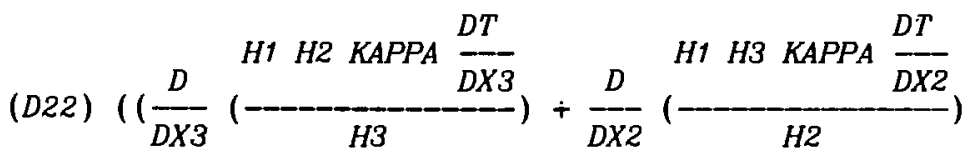

$\left.+\frac{D}{D X 1}\left(-\frac{H 2 H 3 K A P P A \frac{D T}{D X 1}}{H 1}\right)\right) /(H 1 H 2 H 3)$

$\left.-\frac{R H O T\left(\frac{D}{D X 3}(H 1 H 2 V 3)+\frac{D}{D X 2}(H 1 H 3 V 2)+\frac{D}{D X 1}(H 2 H 3 V 1)\right) X X 2}{H 1 H 2 H 3}\right)$

$/(C S U B V R H O)$

(C23) $F[6]: B R H S[1] \$$

(C24) $F[7]: B R H S[2] \$$

(C25) $F[8]: B R H S[3] \$$

PDE_LEVEL_CRADIENTS is nuw called. It performs the same tasks discussed in Example 1, but in this case, it sets up 272 gradient definitions. This function call completes all of the preparations necessary to transform each equation into its finite dimensional counterpart, with the exception of initializing the transformed equation lists:

(C26) MIDS:STARTS:ENDS:[]\$

A MACSYMA iteration loop very similar to the corresponding block of code in 
Example 1 accomplishes this transformation.

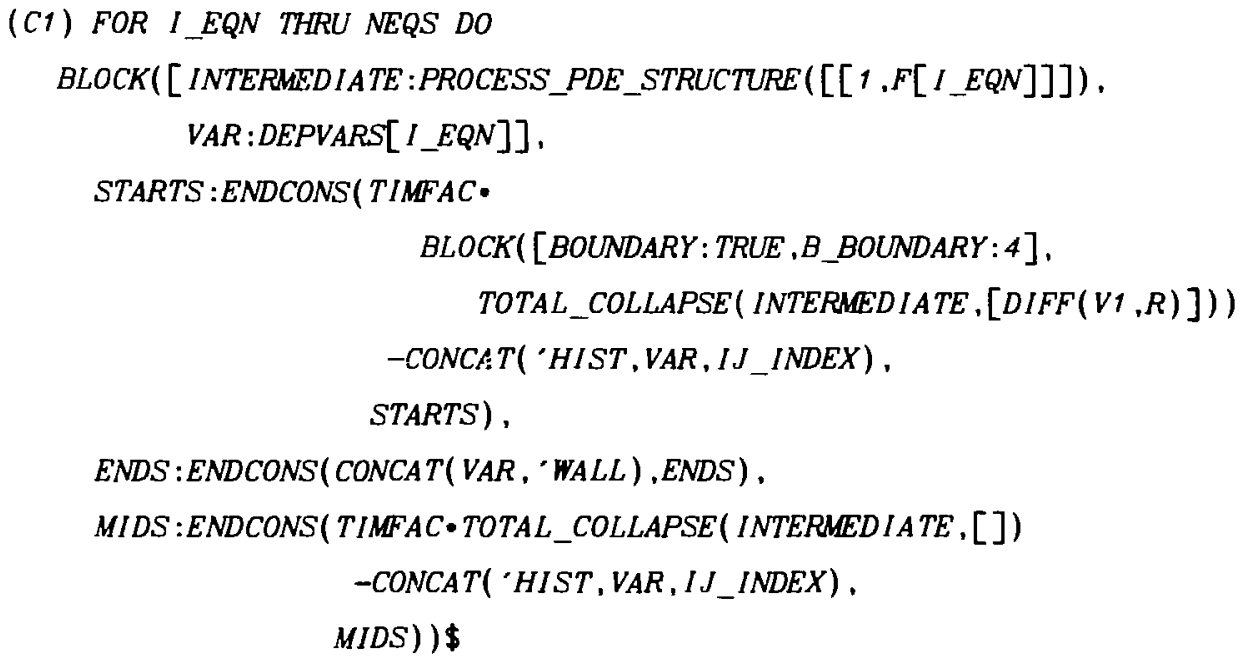

In the structured input to PROCESS_PDE_STRUCTURE, $F\left[I_{-} E Q N\right]$. selects the right-hand side of the equation designated by $I_{-} E Q N$. Also, VAR is set to the dependent variable for which that equation is being solved.

The results are now large and will lead to yet much larger expressions in the Jacobian calculation. To see exactly how large, we let MACSYMA display the equation I ists:

(C2) MIDS;

(D2) $[T I M F A C(-P R H O(I, J) \quad V 3 D Z P(K) \quad X X 5$

$-\operatorname{CVMCP}(\operatorname{RODZM}(K), \operatorname{RODZP}(K),-V 3(I, J)\} V 3(I, J) X X 5$

- PRHO $(I, J) \operatorname{V1DRP}(K) X X 5-\operatorname{CVMCP}(R O D R M(K), R O D R P(K),-V 1(I, J))$ 


$$
\left.V 1(I, J) X X 5-\frac{R H O(I, J) V 1(I, J) X X 5}{R(I)}\right)-\operatorname{HISTRHO}(I, J),
$$

$T I M F A C\left(-T D R P(K) X X 9-\frac{R O D R P(K) T(I, J) X X 9}{P R H O(I, J)}\right.$

$$
\begin{aligned}
& +\frac{V 2(I, J)^{2} X X 8-B 2(I, J)^{2} X X 12}{R(I) R H O(I, J)} \\
& -\frac{C V M G P(V 1 D Z M(K), V 1 D Z P(K),-V 3(I, J)) \text { V3(I.J) XX8 }}{P R H O(I, J)}
\end{aligned}
$$

$$
-\frac{V 1(I, J) \operatorname{CVMGP}(V 1 D R M(K), V 1 D R P(K),-V 1(I, J)) X X 8}{P R H O(I, J)}
$$$$
-\frac{B 3(I, J) \operatorname{CVMCP}(B 3 D R M(K), B 3 D R P(K),-B 3(I, J)) X X 12}{P R H O(I, J)}
$$

$$
+\frac{C M M C P(B 1 D Z M(K), B 1 D Z P(K), B 3(I, J)) B 3(J, J) \quad X X 12}{P R H O(I, J)}
$$$$
\left.-\frac{B 2(I, J) \operatorname{CVMGP}(B 2 D P M(K), B 2 D R P(K),-B 2(I, J)) X X 12}{P R H O(I, J)}\right)-\operatorname{HISTV1}(I, J),
$$

$$
\text { TIMFAC }\left(-\frac{V 1(I, J) V 2(I, J) X X 8-B 1(I, J) B 2(I, J) X X 12}{R(I) \text { RHO }(I, J)}\right.
$$

$$
-\frac{\operatorname{CVMCP}(\operatorname{V2DZM}(K), \operatorname{V2DZP}(K),-V 3(I, J)) V 3(I, J) X X 8}{P R H O(I, J)}
$$

$-\frac{V 1(I, J) \operatorname{CVMCP}(\operatorname{V2DRM}(K), \operatorname{V2DRP}(K),-V 1(I, J)) X X 8}{P R H O(I, J)}$

$$
+\frac{\operatorname{CVMCP}(B 2 D Z M(K), B Z D Z P(K), B 3(I, J)) B 3(I, J) X X 12}{P R H O(I, J)}
$$

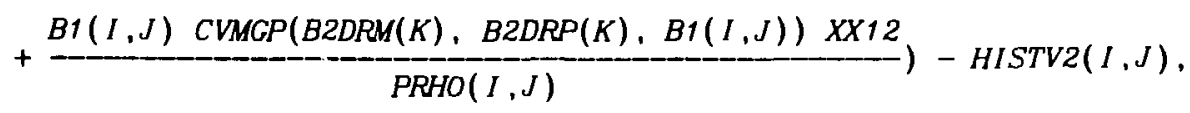


$T I M F A C\left(-\operatorname{TDZP}(K) \times X 9-\frac{R O D Z P(K) T(I, J) X X 9}{P R H O(I, J)}\right.$

$-\frac{V 3(I, J) \operatorname{CVMCP}(V 3 D Z M(K),-V 3 D Z P(K),-V 3(I, J)) X X 8}{P R H O(I, J)}$

$-\frac{V 1(I, J) \operatorname{CVMCP}(V 3 D R M(K), V 3 D R P(K),-V 1(I, J)) X X 8}{P R H O(I . J)}$

$+\frac{B 1(I, J) \operatorname{CVMCP}(B 3 D R M(K), B 3 D R P(K), B 1(I, J)) X X 12}{P R H O(I, J)}$

$-\frac{B 2(I, J) C V M G P(B 2 D Z M(K), B 2 D Z P(K),-B 2(I, J)) \times X 12}{P R^{\prime} H O(I, J)}$

$\left.-\frac{B 1(I, J) \operatorname{CVMGP}(B 1 D Z M(K), B 1 D Z P(K),-B 1(I, J)) X X 12}{P R H O(I, J)}\right)-H I S T V 3(I, J)$.

TIMFAC $(-7(I, J) \quad V 3 D Z P(K) X X 15-T(I, J) \quad V 1 D R P(K) X X 15$

$$
\begin{aligned}
& -\frac{T(I, J) V 1(I, J) X X 15}{R(I)}+\frac{K A P P A T D R M(K) X X 14}{P R H O(I, J) R(I)} \\
& +\frac{K A P P A}{(T D 2 Z(K)+T D 2 R(K)) X X 14}-\operatorname{HISTT}(I, J) \text {. }
\end{aligned}
$$

TIMFAC $(B 1(I, J) \quad C V M C P(V 3 D Z M(K), V 3 D Z P(K), B 1(I, J)) X X 16$

$+C V M G P(B 1 D Z M(K), B 1 D Z P(K), V 3(I, J)) \quad V 3(I, J) X X 16$

$-B 3(J, J) \operatorname{CVMCP}(\operatorname{V1DZM}(K), \operatorname{V1DZP}(K),-B 3(I, J)) X X 16$

$-\operatorname{CVMCP}(B 3 D Z M(K), B 3 D Z P(K),-V 1(I, J)) V 1(I, J) X X 16)-\operatorname{HISTB1}(I, J)$.

TIMFAC $(B 2(I, J) \quad C V M G P(\operatorname{VBDZM}(K), \operatorname{V3DZP}(K), B 2(I, J)) X X 16$

$+C V M C P(B 2 D Z M(K), B 2 D Z P(K), V 3(I, J)) V 3(I, J) X X 16$ 
$-B 3(I, J) \operatorname{CVMCP}(\operatorname{VZDZM}(K), \operatorname{V2DZP}(K),-B 3(I, J)) X X 16$

$-B 1(I, J) \operatorname{CVMGP}(\operatorname{VaDRM}(K), \operatorname{V2DRP}(K),-B 1(I, J)) X X 16$

- $\operatorname{CVMCP}(B 3 D Z M(K), B 3 D Z P(K),-V 2(I, J))$ V2(I,J) XX16

- $\operatorname{CVMCP}(B 1 D R M(K), B 1 D R P(K),-V 2(I, J))$ V2(I,J)XX16

$+B 2(I, J) \quad \operatorname{CVMCP}(\operatorname{VIDRM}(K), \operatorname{V1DRP}(K), B 2(I, J)) X X 16$

$+\operatorname{CVMCP}(B 2 D R M(K), B 2 D R P(K), V(I, J)) V 1(I, J) X X 16)-\operatorname{HISTB2}(I, J)$,

TIMFAC $(-B 1(I, J) \operatorname{CVMCP}(\operatorname{VBDRM}(K), \operatorname{V3DRP}(K),-B 1(I, J)) X X 16$

$-\frac{(B 1(I, J) V 3(I, J)-B 3(I, J) V 1(I, J)) \times X 16}{R(J)}$

- $\operatorname{CVMGP}\left(B 1 D R M(K), B 1 D R P\left(\kappa^{\circ}\right),-V 3(I, J)\right) V 3(I, J) \times X 16$

$+B 3(I, J) \quad \operatorname{CVMGP}(\operatorname{V1DRM}(K), \operatorname{V1DR} P(K), B 3(I, J)) X X 16$

$+\operatorname{CVMGP}(B 3 D R M(K), B 3 D R P(K), V 1(I, J)) V 1(I, J) X X 16)-H I S T B 3(I, J)]$

(C3) STARTS;

(D3) $[$ TIMFAC $(-P R H O(I, J) \operatorname{V3DZP(K)XX5-RODZP(K)} \vee 3(I, J) X X 5$

- $\operatorname{CVMGP}(R O D R M(K), \operatorname{RODRP}(K),-V 1(I, J)) V 1(I, J) X X 5$

$\left.-\frac{R H O(I, J) V I(I, J) X X 5}{R(I)}\right)-H I S T R H O(I, J)$,

TIMFAC $\left(-\operatorname{TDRP}(K) \times X 9-\frac{\operatorname{RODRP}(K) T(I, J) X X 9}{P R H O(I, J)}\right.$ 


$$
\begin{aligned}
& +\frac{V 2(I, J)^{2} X X 8-E 2(I, J)^{2} X X 12}{R(I) R H O(I, J)}-\frac{V 1 D Z P(K) V 3(I . J) X X 8}{P R H O(I, J)} \\
& -\frac{B 3(I, J) \operatorname{CVMCP}(B 3 D R M(K), B 3 D R P(K),-B 3(I, J)) \times X X 12}{P R H O(I, J)} \\
& +\frac{B 1 D Z P(K) B 3(I, J) X X 12}{P R H O(I, J)} \\
& \left.-\frac{B 2(I, J) \operatorname{CVMCP}(B 2 D R M(K), B 2 D R P(K),-B 2(I, J)) X X 12}{P R H O(I, J)}\right)-\operatorname{HISTV1}(I, J),
\end{aligned}
$$

TIMFAC $\left(-\frac{V 1(I, J) V 2(I, J) X X 8-B 1(I, J) B 2(I, J) X X 12}{R(I) R H O(I, J)}\right.$

$$
\begin{aligned}
& -\frac{V 2 D Z P(K) V 3(I, J) X X 8}{P R H O(I, J)} \\
& -\frac{V 1(I, J) \operatorname{CVMGP}(V 2 D R M(K), V 2 D R P(K),-V 1(I, J)) X X 8}{P R H O(I, J)}
\end{aligned}
$$

$$
+\frac{B 2 D Z P(K) B 3(I, J) X X 12}{P R H O(I, J)}
$$$$
\left.+\frac{B 1(I, J) \operatorname{CVMCP}(B 2 D R M(K), B 2 D R P(K), B 1(I, J)) X X 12}{P R H O(I, J)}\right)-\operatorname{HISTV2}(I, J) .
$$

$$
\text { TIMFAC }\left(-\operatorname{TDZP}(K) X X 9-\frac{\operatorname{RODZP}(K) T(I, J) X X 9}{P R H O(I, J)}-\frac{V 3(I, J) \operatorname{VBDZF}(K) X X 8}{P R H O(I, J)}\right.
$$$$
-\frac{V 1(I, J) \operatorname{CVMGP}(\operatorname{VBDRM}(K), \operatorname{V3DRP}(K),-V 1(I, J)) X X 8}{P R H O(I, J)}
$$

$$
+\frac{B 1(I, J) \operatorname{CVMCP}(B 3 D R M(K), B 3 D R P(K), B 1(I, J)) X X 12}{P R H O(I, J)}
$$

$$
\left.-\frac{B 2(I, J) B 2 D Z P(K) X X 12}{P R H O(I, J)}-\frac{B I(I, J) B 1 D Z P(K) X X 12}{P R H O(I, J)}\right)-H I S T V 3(I, J) .
$$




$$
\begin{aligned}
& \text { TIMFAC }\left(-T(I, J) V 3 D Z P(K) X X 15-\frac{T(I, J) V 1(I, J) X X 15}{R(I)}\right. \\
& \left.+\frac{K A P P A}{P D D R M(K) X X 14}+\frac{K A P P A}{P R H O(I, J) R(I)}+\frac{(T D 2 Z P(I)+T D 2 R(K)) X X 14}{P R H O(I, J)}\right) \\
& -H I S T T(I, J), T I M F A C(B 1(I, J) V 3 D Z P(K) X X 16+B 1 D Z P(K) V 3(I, J) X X 16 \\
& -B 3(I, .) V 1 D Z P(K) X X 16-B 3 D Z P(K) V 1(I, J) X X 16)-H I S T B 1(I, J),
\end{aligned}
$$$$
\text { TIMFAC }(B 2(I . J) V 3 D Z P(K) X X 16+B 2 D Z P(K) V 3(I . J) X X 16
$$$$
-B 3(I, J) \quad V 2 D Z P(K) X X 16-B 1(I, J)
$$$$
C V M G P(\operatorname{VIDRM}(K), \operatorname{V2DRP}(K),-B 1(I, J)) X X 16-B 3 D Z P(K) \operatorname{V2}(I, J) X X 16
$$$$
\text { - } C V M C P(B 1 D R M(K), B 1 D R P(K),-V 2(I, J)) V 2(I, J) X X 16
$$$$
+\operatorname{CVMCP}(B 2 D R M(K), B 2 D R P(K), V 1(I, J)) V 1(I, J) X X 16)-\operatorname{HISTB2}(I, J),
$$$$
\text { TIMFAC }(-B 1(I, J) \text { CVMCP }(\operatorname{ViDRM}(K), \operatorname{V3DRP}(K),-B 1(I, J)) X X 16
$$$$
-\frac{(B 1(I, J) V 3(I, J)-B 3(I, J) V 1(I, J)) \times X 16}{R(I)}
$$$$
\text { - } C V M C P(B 1 D R M(K), B 1 D R P(K),-V 3(I, J)) V 3(I, J) X X 16
$$$$
+\operatorname{CVMCP}(B 3 D R M(K), B 3 D R P(K), V 1(I, J)) V 1(I, J) X X 16)-\operatorname{HISTB} 3(I, J)]
$$

(C4) ENDS;

(D4) [RHOWALL, V1WALL, V2WALL, V3WALL, TWALL, B1WALL, B2WALL, B3WALL]

The artificially simple boundary conditions at the top boundary serve to emphasize, by contrast, how large the other expression lists are. 


\subsubsection{Jacobian Calculation and Code Generation}

With the discretization phase complete, the Jacobian calculation and code generation are performed precisely as before.

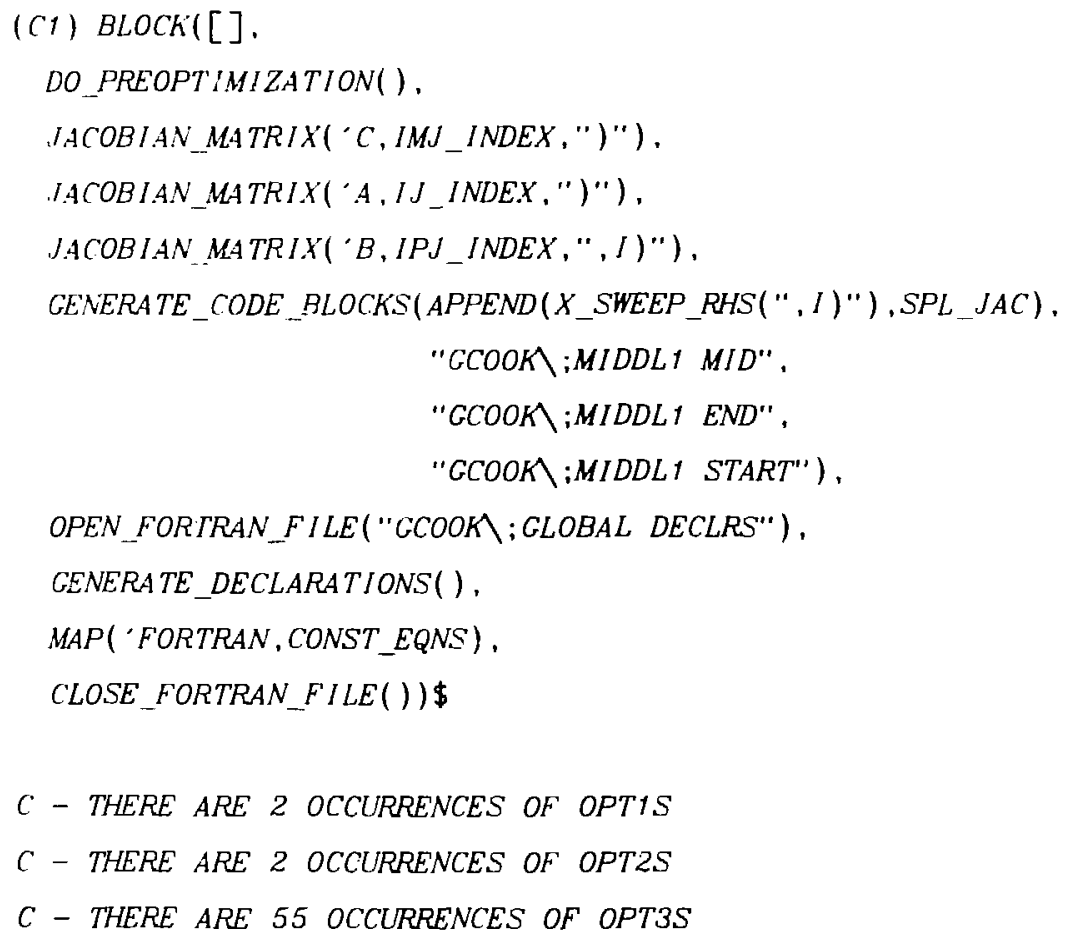

Because of the volume of FORTRAN output generated for this model, the listings of each of the four output files used for FORTRAN output in this 
calculation are relegated to Appendix E. It is most important to realize, however, that these FORTRAN code segments are not the complete set required to actually frame a computer code for solving the simple MHD model. The complete set would be perhaps four or five times larger, and was not computed because the part that was constructed already taxed the available resources of the DEC -10 computer to the limit.

\subsection{The Complex MHD Model}

While the general flow of the computation in both Examples 1 and 2 is the same as was used for the complex MHD model calculation, much has been left out. It has been left out because most of the remaining detail is opaque or not particularly illuminating. Nevertheless, there are some operating system issues of general interest which are not opaque, and without which the large MACSYMA computation could not have been done.

First, as noted earlier, the constraints imposed by a time sharing environment prohibit the running of a very long job ( 10 hours) without interruption. This may not be true on the powerful personal computers of the future. As a result of the time limitation, the job was segmented into eight separate sections. Each was made into a function named $M A I N$, and each set a global variable NEXT_PHASE to the name of the file containing the next computational phase. Thus, when a given function MAIN had completed its lask; i.e., when a phase of the computation was done, the controlling MACSYMA function 
could initiate the next portion of the run by simply loading the file designated by NEXT_PHASE and then invoking the same function it called to run the last section.

However, in the real world, the unpredictable behavior of computer systems requires that partial results be saved so as to be able to recover from system crashes. Unfortunately. these crashes are not out of the ordinary; several were encountered during the large MACSYMA run. Therefore, before initiating the next phase of the computation, the job state must be saved. And because of the possibility that the computer could crash while this operation is underway, a backup of the job state was also kept. The fact that a MACSYMA SAVE operation requires such a long $t$ ime for any sizeable amount of information increases the probability of a crash during that operation. Thus, great pains were taken to ensure that the latest job state was safa, not only from system crashes, but also from other unpredictable behavior such as bugs in the MACSYMA program or bugs in the systems software.

As mentioned above, the SAVE command is very inefficient. Indeed, in tests I conducted. I determined that saving the job state at randomly selected junctures in the computation could actually consume hours of computer $t$ ime. The segmenting of the job was therefore chosen very carefully to minimize the amount of information which had to be saved to allow the computation to proceed at a later time. And while this strategy may not be essential on other computer 
systems, minimizing the amount of information required to continue an interrupted computation is a good practice. 


\section{CHAPTER 7}

\section{CONCLUSIONS}

\subsection{Completed Code Work}

As mentioned in Chapter 1, this effort did not produce a working code. The development of all major parts of the main code was completed, as well as parts of the preprocessor. Included in the main code development were four large-scale algebraic computations and several smaller-scale ones. The first, and by far the largest of the algebraic computations, was accomplished on the MULTICS computer at MIT. This computation involved the discretization of the MHD equations and calculation of all of the Jacobian elements called for by the solution technique of Chapter 4. Although the calculation took 45 hours of computer time, the demands on the $t$ imeshared MULTICS computer frequent ly delayed the computation so that it was completed only after five weeks. About 54,000 lines of FORTRAN code were generated, much of it involving many continuation lines. Because of the large number of continuation lines, the coding is quite 
dense, and therefore, the 54,000 lines of FORTRAN compare only to a conventional code of much larger size.

The second major algebraic task was the searcis for compatible boundary conditions for the spline variables. These calculations were done on the MIT-MC DEC-10 computer, as were all remaining calculations discussed in this section. Consuming about two hours of computer time, the results of this search are found in Appendix B. Because of the difficulty of the computation, calculating the truncation errors took a good percentage of the computer time.

Assuming that the currents in the driving coils are known, the external field, together with its derivatives, can be found in the plasma domain. The third major algebraic task was to generate an optimal vectorizing code segment for numerically computing $e^{e x t}$ and its derivatives, given that a vectorizing code segment based on the theta nome hybrid methods of Appendix $D$ is available for computing $K$ and $E$. This algebraic computation required about 30 minutes of computer $t$ ime. Some of the second derivatives of $\boldsymbol{e}^{\mathrm{x} t}$ do not appear in the expanded MHD equations, and so the computation was repeated for the minimal set of external field quantities.

Formal discretization of the coil equations constitutes the fourth major algebraic task. As stated in Chapter 5, because these equations are integro-differential equations, a completely different set of algebraic processing tools had to be developed to process them. Also, because of the variety of configurations the driving coil circuits can take in the Topolotron 
experiment, a number of different sets of integro-differentıal equations were processed for inclusion in the final code. With this volume of algebraic work, the calculation again took about 30 minutes of computer time.

Several short algebraic computations also contributed to the code generation effort. Most prominent among them was the inversion of the linear iystem for the bi-quintic interpolant described in Appendix $C$, and the generation of optimal vectorizable code from the result. This involved roughly 15 minutes of computer time. Generation of optimal vectorizable code segments for all methods of finding $K$ and $E$ in Appendix D was more difficult to program. but required only about 10 minutes of computer time. The specific code segment settled upon for the final MHD code took less than a minute to generate. Finally, the code to compare the improvement in accuracy of the partition function for the deuterium molecule by including more and more correction terms was constructed in about $\mathrm{f}$ ive minutes of computer $t$ ime.

The nature of other parts of the MHD code made it easier to program them by hand. Two major code pieces in this category are the block tridiagonal solver presented in Chapter 4, and the elaborate product integration scheme for first kind Fredholm integral equations with singular kernels discussed in Chapter 3.

All of these code segments reside in the archives at the National Magnetic Fusion Energy Computer Center (NMFECC). Although further work in 
assembling the code pieces was begun, serious errors in the CRAY FORTRAN compilers forstalled any significant progress.

\subsection{Remaining Code Work}

The first thing that remains is to finish the operating environment for the code. That is, the code will need a halting and restarting capability because simulations of complex phenomena take many hours of CRAY-1 time. Also, although the larger part of the pre-processor or code-initialization phase is in place, the logic to read an input deck specifying the physical problem to be run (initial conditions, etc.) has not been written. Both of these projects require detailed knowledge of the Cray time sharing system (CTSS) at Livermore or of whatever other operating system is in use. With this level of skill, these projects could be done in two man-months.

The post-processor, or graphical analysis code, was not begun. This is a large project if it is to be done correctly for the bi-quintic polynomial splines and the quintic B-splines used to represent the main physics variables. On the other hand, simple graphical capability could be implemented with far less effort. In either event, this work would involve several months of effort for an experienced user of high-level graphics.

Beyond this extra coding work, a considerable effort lies ahead to get the existing vectorizable code segments to first compile and then vectorize. Even familiarity with FORTRAN compilers in general and the CRAY FORTRAN 
compilers in particular may not suffice in this instance because of the logic errors that currently exist in the CRAY compilers. While the errors may be removed shortly, major adjustments to the DO-loop structure of the MHD code st 111 make this a several-month project.

After the pre- and post-processing codes are debugged, the main code must be tackled. This is a formidable undertaking. Indeed, physics coding errors were discovered in two of the major codes in use at Lawrence Livermore National Laboratory after they had been in production for several years. So an understanding of how to proceed with the debug phase is not currently available. Only considerable insight will help the computational physicist track down the bugs .

Once a working code has been obtained, it remains to ask if the code can economically solve the physics problem of interest. If not, we may not be able to answer physically interesting questions within our budget restrictions. Two criteria were used in the selection of the numerical techniques chosen for the MHD code. The first criterion was that the technique had to be numerically stable. But just as important, the second criterion was that the technique had to allow fairly rapid computation. At this writing, it is not known if the second criterion is always satisfied because it is not known whether the one-leg time integration method will actually turn out to perform in a second-order manner. If it does, the numerical methods used in the MHD code will not limit the code's performance, and the code should be competitive with LASNEX in terms 
of speed. It is very difficult to make any concrete statements about the speed of the computation because of the very different numerical methods used in this and other major codes. That is, convective and conservation forms lead to different code sizes. Moreover, the fully implicit treatment of the MHD equations dramatically affects the code size in comparison with codes using a time-operator splitting approximation. Outside of the numerical issues, there are questions of how well the FORTRAN compilers can vectorize the competing codes. Unfortunately, they cannot do very well on the new MHD code because of internal limitations on how large a code block can be and still vectorize. Presently, this limitation is of the order of 50 code lines, far smaller than the 1000 line DO-loops (and larger) which were generated by the MHD algebra calculation performed as part of this study. Hence, only experience with a working version can provide reasonable estimates of the costs of running the new MHD code.

\subsection{Simulation Studies}

The intent of this research was to improve our understanding of how the plasma in the present Topolotron experiment develops in time. Since the computer code to perform these simulations has not been completed, only one preliminary question is answered: What computer experiments should be performed if the simulation code were to become available? The most interesting initial experiment would be to follow the plasma through its lifetime for the most 
successful set of initial conditions observed in actual operation of the experiment. Then it would be instructive to see how much of the experimental data could be duplicated by other partial simulation runs. Experimental corroboration, by the way, would prove to be one of the most concrete validations of the simulation code if plasma conditions were well within the regime of validity of the MHD model and neglected effects were indeed negligible. Finally, a search for optimal initial conditions might be conducted or, if that proved fruitless, exploration of other experimental configurations begun.

While comparison against experiment is one of the most reliable guides, much of the data taken in plasma physics experiments represents integrated or averaged data instead of the local information available from the computer simulation. Moreover, there is comparatively little data taken from these experiments. One means of providing much more information concerning the real behavior of the plasma is the use of two-dinensional reconstruction techniques. ${ }^{24}$ This numerical inversion process uses projected density information to reproduce the local values of the density in the interior of the plasma. If the data is taken at short enough intervals, the reconstructed density information could be numerically differentiated to obtain two of the one-fluid velocity components in the plasma. This informa:ion could then be coupled with extensive probing of the $B$-field structure to give very detailed comparisons between a computer simulation and the experiment. When the computer 
code is fully debugged, such comparisons could be used as well to make evaluations of the physical model in the code.

\subsection{Improved MHP Model}

As was implied earlier. it is crucial that the experiment being modelled numerically be adequately represented by the physical model, and there are questionable areas in the model developed in Chapter 2 . As discussed there, the most important improvement would be to find ionization populat,ions and radiation losses from non-LTE rate equations. Equally important. impurities must be modelled because of the dramatic effect they have on cooling the plasma. It is conceivable that a good model for these particles could be added without requiring an entirely new set of MHD equations, but the theory has not been done. While it is hard to assess just how important microturbulence-enhanced transport is in a Topolotron device, additional ad hoc terms should be added to the model to account for the best-understood enhancement mechanisms. This should not be as important as the previous two modifications, however.

\subsection{Better Numerical Techniques}

Because the Topolotron is a pinch device, numerous shocks will traverse the plasma during its development. Shock development implies numerous regions of steep gradier، or rapid change in the values of the dependent variables. Even the higher-order spline basis functions used here will be incapable of 
resolving the shock structure, especially since the shocks are moving through the plasma. Therefore, a means of hybridizing the "compact" spline methods discussed in Chapter 3 with an AMM or AMR technique appears to offer the best hope for remedying this deficiency, because those techniques are designed specifically to resolve the regions of large gradient.

In the coil calculation, it would be of interest to extend the numerical approximation of the current distribution in the coils to a fully two-dimensional one, treating the surface currents as in a boundary layer problem. This extension could be accomplished by one of two possible approaches. First, an AMM technique could be used to have the computational mesh adapt to the presence of the boundary layer. This technique would undoubtably lead to an exponential distribution of grid points in the direction normal to the surface. Because the crowbarred capacitors cause the current to become more like a direct current, the boundary layer will diffuse with time and the need for the exponentially-graded mesh would disappear. Again, the AMM technique would automatically (and optimally) adapt to this new set of circumstances. The second method would involve a priori knowledge of the beginnirig of the boundary layer. Inside the boundary layer, the current would be expanded in appropriate exponential basis functions (or rational approximations thereof), and in the interior region, standard splines would be used. A matching condition at the beginning of the buundary layer would then complete the problem statement. While potentially less complex than a full AMM 
treatment, this second method requires an ability to determine where the matching condition should be applied. It could be that an iterative method of solving the problem over and over again would be required to locate the correct position.

\subsection{Computer Assisted Algebra}

Dramatic gains have been made in relieving the physicist of much arduous, error-prone hand algebra. The primary, common-sense techniques by which these gains have been achieved will indubitably continue to be used for some time. However, there are improvements which are possible. Just as the internal representation is $f$ ar from perfect in MACSYMA, the representations used here can also be improved. For example, the inclusion of formal subscripting information in the print name of the variable is an awkward choice for efficiently keeping track of the subscript. It is awkward because of the changes which need to be made to the subscript in each specific code generation context. So, a better means of efficiently carrying this information along in the calculation should be sought.

Insofar as the basic algebra functions developed here are concerned, there is a!so potential for improvement. GCFAC, the general collection of fast factoring algorithms, certainly needs some multivariate multinomial factoring capability. Finding cheap ways of doing this will be very difficult. Also important will be some heuristics to help guide when such an algorithm would be 
applied, for if it were applied indiscriminately, significant speed degradation would result as it does with the present FACTOR function in MACSYMA.

In Chapter 6, it was pointed out that the structuring of the formal PDEs as required by PROCESS_PDE_STRUCTURE was done manually. There is no reason that a MACSYMA-level function could not be written to automate this step and further eliminate the possibility for human error. Also, the discretization of the time derivatives could be incorporated into this new function.

Another area which would benefit from computer assisted algebra techniques is the spatial discretization method of flux-form finite differencing. This method is sufficiently different from the collocation method that several thousand lines of MACSYMA and LISP code would require rewriting. But the benefit of having this capability would be to facilitate the rapid comparison of higher-order spline collocation and flux-form finite differencing methods when applied to non-trivial physical models.

\subsection{Simulation Codes}

While the bulk of the coding required for the physics in Chapter 2 was automatically generated, there are parts of the final simulation code which were not automatically generated. These were principally the execution control logic and processing and post-processing capability. To see exactly what remains to be done, let us break down a simulation code into its principal components:

1. Storage layout and FORTRAN declarations. 
2. Memory management $\log$ ic.

3. Pre-processing or code initialization.

4. Functions and subroutines to compute everything implied by Chapters 2 through 4 .

5. Operating system-like cude to control execution.

6. Post-processing of the results, including graphics.

In category 1, storage layout and FORTRAN declarations, all of the declaration-type information needed to do storage layout is carried internally by our MACSYMA functions, and the functionality to translate this into DIMENSION, COMMON, and POINTER statements has been provided. However, very limited typing information is developed by these functions. In particular, the typing of some real arrays which are intrinsically of integer type had to be done by hand. Adding typing functions to MACSYMA would be trivia!

The memory management implied by category 2 is a developing area. For example, not all vectors and arrays in our application are memory managed, while in principle, all of them could be. The decision of which ones were to be memory managed was built into the storage layout function, leaving the user with no flexibility. In addition, only some of the actual run-time memory management function calls were automatically generated. Nevertheless, all of these deficiencies could be readily corrected with little additional effort. 
Category 3, pre-processing or code initialization, involves two aspects. First, there is the logic to process an input deck giving details as to what physics problem to run. No automation work was performed in this area at all. Secondly, in a large-scale simulation code, it is important to compute once and for all all constant expressions (found by REDUCE CONSTS, for example) and time-invariant quantities. One set of time-invariant quantities in this resiarch was the matrix of singular integrals arising in the $I$ and $C$-coil calculation. Every constant expression and time-invariant quantity found during the code development process was saved for inclusion in this pre-processing step of the simulation code. However, the nature of the singular integral evaluation was such that the code to assemble the matrix of those integrals had to be constructed by hand.

The bulk of the coding in any large-scale physics code will be found in category 4, numerical functions and subroutines; it remains the overwhelming reason for computer assisted algebra. There were, however, parts of this category which were done by hand. These were the nonlinear equation solver and the code selecting the size of the next time integration step. They were not automated because the theory in each area is not well-established. Once again. they could be automatically generated if there were sufficient interest.

Category 5, execution control, involves the logic which decides when the simulation should be stopped, how to stop it gracefully and restart it later from a saved state. Another very important part of the code's operating system 
would be debugging logic; i.e., functions which would allow a simulation to be interrupted and have physics and other variables displayed at a user terminal. Because of the importance of the execution controller, much work has gone into developing significant power in the controller. For example, the LASNEX ${ }^{90}$ controller is many thousands of code lines long. While no computer assisted code generation was done for this category either, it is clear that a large amount of effort for each new code could be saved if there were automation facilities available. Moreover, the simulation code could be made smaller by having decisions made at a symbolic level as to what specific operating system functions would be required. Nonetheless, it is not quite as simple as it sounds, because the code in this execution controller and debugger will be machine-dependent. That is, there is no standard computer operating system in use in large-scale computing environments such as on the CRAY-1. Consequently. each new computer will require that the "templates" used by the code generator at the symbolic level be modified to accomodate the new host operating system. And it could be worse than that. One solution to this would be to have a two-level tool kit instead of the single-level one implied by the above discussion. In this tool kit would be a top-level set of machine-independent functions describing succintly what a large-scale simulation code might require. The bottom level would consist of the details of those functions and how they interface to the host operating system. The benefit to be derived from this technique is that only the bottom level would need to be rewritten when any change in computer system occurred. 
The last category, that dealing with post-processing, is again one where large amounts of manpower have been expended. And although there are some graphics standards, there is no portable graphics library which is widely accepted. Consequently, this area is also a moving target and would benef it from a sirategy at the symbolic level similar to that described above for the controller logic. There is more to the post-processing phase than just the graphics that would benefit from this approach. Specifically, the means of specifying what part of the "snapshots" of the plasma states will be analyzed and how the data reduction will occur both involve decision logic and host operating system-dependent logic. The data reduction must be driven via some input specifications, so once again more host operating system features arise. If all of these arcane details could be concentrated in some machine-dependent. low-level modules, everything else required by the simulation code could be generated at the symbolic level (in MACSMMA or LISP). The wide variety of twoand three-dimensional plotting capability which simulation codes might require make the development of the correct modules a complex project. Several years of evolving ideas, using input from a wide base of computational physicists, would eventually yield a very worthwhile post-processing product. 


\section{APPENDIX A}

\section{A VECTOR IDENTITY}

From the vector identity

$$
\nabla \times(c \times d)=c(\nabla \cdot d)-d(\nabla \cdot c)+(d \cdot \nabla) c-(c \cdot \nabla) d
$$

we can write

$$
\nabla \times[\mathbf{a} \times(f \mathbf{b})]=\boldsymbol{a}[\boldsymbol{\nabla} \cdot(f \mathbf{b})]-f \mathbf{b}(\nabla \cdot \mathbf{a})+[(f \mathbf{b}) \cdot \nabla] \mathbf{a}-(\mathbf{a} \cdot \nabla)(f \mathbf{b})
$$

for an arbitrary function $f$ and arbitrary vectors $\bullet, b, c$, and $d$. The ieft-hand side can be rewritten

$$
\nabla \times[a \times(f b)]=-\nabla \times[(f b) \times a]=f \nabla \times(a \times b)+(\nabla f) \times(a \times b)
$$

The first term in the final expression is $f$ times the left side of (Al), while the second term can be written 


$$
(\nabla f) \times(\mathbf{a} \times \mathbf{b})=[(\nabla f) \cdot \mathbf{b}] \mathbf{a}-[(\nabla f) \cdot \mathbf{a}] \mathbf{b} .
$$

With the result

$$
\nabla \cdot(f b)=f \nabla \cdot b+b \cdot(\nabla f)
$$

and some further rearrangement. (A2) reduces to

$$
(\mathbf{a} \cdot \nabla)(f \mathbf{b})=f(\cdot \mathbf{a} \cdot \mathbf{\nabla}) \mathbf{b}+\mathbf{b}(\mathbf{a} \cdot \nabla f)
$$




\section{APPENDIX B}

\section{SPLINE BOUNDARY CONDITIONS}

In order to develop highly accurate boundary conditions for the spline variables approximating spatial derivatives of the dependent variables, it is essential to find the truncation error implied by the spline relations ( 3.7 ) for the first derivative coefficient, $m_{j}$, as well as the spline relation (3.9) for the spline second derivative coefficient, $K_{j}$. Once this information is available for $K_{j}$, however, a Taylor series expansion immediately gives the result for $m_{j}$.

To determine the truncation error of $K_{j}$, we assume that it can written as a Taylor series in $u_{j}$ with unknown coefficients:

$$
K_{j}=\sum_{i \neq 0} a_{i} \frac{\partial^{i} u_{j}}{\partial x^{i}}
$$

By plugging this expansion into (3.9). Taylor expanding about the $j$ th $g r i d$ point, and gathering all terms to one side of the equation, a new series of the 
form (B1) is obtained. By finding the lowest order derivative of $u$, whose coefficient contains $a_{o}$, that coefficient can be set to zero and the resulting equation solved for $a_{0}$. Substitution of the value of $a_{0}$ into the rest of the series. finding the lowest-order derivative of $u_{j}$ whose coefficient contains $a_{,}$, setting that coefficient to zero and solving for $a_{1}$, all of these steps constitute the second part of the calculation. This process can be repeated as many times as needed to determine as many of the $a_{2}$ as desired. With this procedure, it was found that

$$
\begin{aligned}
K_{j}= & \frac{\partial^{2} u_{j}}{\partial x^{2}}-\frac{h_{j+1}^{2}-h_{j} h_{j+1}+h_{j}^{2}}{12} \frac{\partial^{4} u_{j}}{\partial x^{4}}-\frac{\left(h_{j+1}-h_{j}\right)\left(h_{j+1}+2 h_{j}\right)\left(2 h_{j+1}+h_{j}\right)}{180} \frac{\partial^{5} u_{j}}{\partial x^{5}} \\
& +\frac{7 h_{j+1}^{4}-16 h_{j} h_{j+1}^{3}+21 h_{j}^{2} h_{j+1}^{2}-16 h_{j}^{3} h_{j+1}+7 h_{j}^{4}}{1080} \frac{\partial^{6} u_{j}}{\partial x^{6}} \\
& +\frac{\left(h_{j+1}-h_{j}\right)\left(h_{j+1}+2 h_{j}\right)\left(2 h_{j+1}+h_{j}\right)\left(11 h_{j+1}^{2}-10 h_{j} h_{j+1}+11 h_{j}^{2}\right)}{11340} \frac{\partial^{7} u_{j}}{\partial x^{7}} \\
& +o\left(h^{6}\right)
\end{aligned}
$$

where $h=\max \left(h_{j}, h_{j+1}\right)$. Then, using this result, it was found that 


$$
\begin{aligned}
m_{j}= & \frac{\partial u_{j}}{\partial x}-\frac{h_{j} h_{j+1}\left(h_{j+1}-h_{j}\right)}{24} \frac{\partial^{4} u_{j}}{\partial x^{4}}-\frac{h_{j} h_{j+1}\left(h_{j+1}^{2}-h_{j} h_{j+1}+h_{j}^{2}\right)}{180} \frac{\partial^{5} u_{j}}{\partial x^{5}} \\
& +\frac{h_{j} h_{j+1}\left(h_{j+1}-h_{j}\right)\left(7 h_{j+1}^{2}-5 h_{j} h_{j+1}+7 h_{j}^{2}\right)}{2160} \frac{\partial^{6} u_{j}}{\partial x^{6}} \\
& +\frac{h_{j} h_{j+1}\left(44 h_{j+1}^{4}-23 h_{j} h_{j+1}^{3}+12 h_{j}^{2} h_{j+1}^{2}-23 h_{j}^{3} h_{j+1}+44 h_{j}^{4}\right)}{45360} \frac{\partial^{7} u_{j}}{\partial x^{7}} \\
& +\mathcal{O}\left(h^{7}\right) .
\end{aligned}
$$

Observe that this is not the same truncation error as was given by Rubin and Khosla. ${ }^{99}$ This is due to the fact that they computed it from a different spline relationship for $m_{j}$. The relations we are using for $m_{j}$ are evidently slightly less accurate, but they do permit the elimination of $m_{j}$ from the MHD equations.

As the truncation error for $G_{j}, \hat{l}_{i, j}, \hat{K}_{i, j}, \hat{L}_{i, j}$, and $T_{i, j}$ are also required below, these will be developed in turn. The truncation error for $G$, is found using (B2) in equation (3.8) and Taylor expanding about the $j$ th grid point. The result is

$$
\begin{aligned}
G_{j}= & \frac{\partial^{2} u_{j}}{\partial x^{2}}+\frac{\left(h_{j+1}-h_{j}\right)\left(h_{j+1}-3 h_{j}\right)\left(3 h_{j+1}-h_{j}\right)}{180} \frac{\partial^{5} u_{j}}{\partial x^{5}} \\
& +\frac{7 h_{j+1}^{4}-16 h_{j} h_{j+1}^{3}+21 h_{j}^{2} h_{j+1}^{2}-16 h_{j}^{3} h_{j+1}+7 h_{j}^{4}}{1080} \frac{\partial^{6} u_{j}}{\partial x^{6}} \\
& +\frac{\left(h_{j+1}-h_{j}\right)\left(h_{j+1}^{4}+56 h_{j} h_{j+1}^{3}-48 h_{j}^{2} h_{j+1}^{2}+56 h_{j}^{3} h_{j+1}+h_{j}^{4}\right)}{11340} \frac{\partial^{7} u_{j}}{\partial x^{7}} \\
& +\mathscr{O}\left(h^{6}\right) .
\end{aligned}
$$


This result differs from Rubin and Khosla's result 102 because of an incorrect sign in their version of (B2). This underlines the need to have reliable computer algebra tools to perform these mundane tasks without human errors. Also, the calculations become more and more difficult in this appendix. Consequently, because of our finite capability to perform algebra operations correctly, all of this work was done with MACSYMA.

Next, to find the trunca on error for $\hat{l}_{2,}$, , the method described above was used on the spline relationship (3.11) to obtain

$$
\begin{aligned}
\hat{l}_{i, j}= & \frac{\partial^{2} u_{i, j}}{\partial x_{1} x_{2}}-\frac{h_{i} h_{i+1}\left(h_{i+1}-h_{i}\right)}{24} \frac{\partial^{5} u_{i, j}}{\partial x_{1}^{4} \partial x_{2}}-\frac{k_{j} k_{j+1}\left(k_{j+1}-k_{j}\right)}{72} \frac{\partial^{5} u_{i j}}{\partial x_{1} \partial x_{2}^{4}} \\
& -\frac{h_{i} h_{i+1}\left(h_{i+1}^{2}-h_{2} h_{i+1}+h_{i}^{2}\right)}{180} \frac{\partial^{6} u_{i, j}}{\partial x_{1}^{5} \partial x_{2}}-\frac{k_{j} k_{j+1}\left(k_{j+1}^{2}-k_{j} k_{1+1}+k_{2}^{2}\right)}{180} \frac{\partial^{6} u_{2,}}{\partial x_{1} \partial x_{2}^{5}} \\
& +\frac{h_{i} h_{i+1}\left(h_{i+1}-h_{i}\right)\left(7 h_{i+1}^{2}-5 h_{i} h_{i+1}+7 h_{2}^{2}\right)}{2160} \frac{\partial^{7} u_{2, j}}{\partial x_{1}^{6} \partial x_{2}} \\
& -\frac{k_{2} k_{3+1}\left(k_{j+1}-k_{j}\right)\left(3 k_{j+1}^{2}-5 k_{j} k_{j+1}+3 k_{j}^{2}\right)}{2160} \frac{\partial^{7} u_{2, j}}{\partial x_{1} \partial x_{2}^{6}} \\
& +o\left(h^{6}, k^{6}\right) .
\end{aligned}
$$

As noted earlier, $H_{2,}$ is the counterpart for $G_{2}$, with respect to the second coordinate variable. All that is needed to find its truncation error is to replace $x_{1}$ with $x_{2}$ and $h_{2}$ with $k$, in formula (B4). Similarly, $\hat{L}_{2}$, will bear the same reiationship to $\hat{K}_{2, j}$. except in this case $h_{i}$ and $k$, both appear and 
must be interchanged. We therefore give only the truncation error for $\hat{K}_{i,}$, as derived from $(3.15)$ :

$$
\begin{aligned}
\hat{K}_{i, j}= & \frac{\partial^{3} u_{i, j}}{\partial x_{1}^{2} x_{2}}-\frac{k_{j} k_{j+1}\left(k_{j+1}-k_{j}\right)}{72} \frac{\partial^{6} u_{i, j}}{\partial x_{1}^{2} \partial x_{2}^{4}}+\frac{\left(h_{i+1}-h_{i}\right)\left(h_{i+1}-3 h_{i}\right)\left(3 h_{i+1}-h_{i}\right)}{180} \frac{\partial^{6} u_{i, j}}{\partial x_{1}^{5} \partial x_{2}} \\
& -\frac{k_{j} k_{j+1}\left(k_{j+1}^{2}-k_{j} k_{j+1}+k_{j}^{2}\right)}{180} \frac{\partial^{7} u_{i, j}}{\partial x_{1}^{2} \partial x_{2}^{5}} \\
& +\frac{7 h_{i+1}^{4}-16 h_{i} h_{i+1}^{3}+21 h_{i}^{2} h_{i+1}^{2}-16 h_{i}^{3} h_{i+1}+7 h_{i}^{4}}{1080} \frac{\partial^{7} u_{i, j}}{\partial x_{1}^{6} \partial x_{2}} \\
& +\mathscr{O}\left(h^{5}, k^{5}\right) .
\end{aligned}
$$

The relationship (3.15) for $\widehat{K}_{i, j}$ was deduced from the tridiagonal spline relationship for $m_{j}$,

$\frac{h_{j+1}}{h_{j}} m_{j-1}+2\left(1+\frac{h_{j+1}}{h_{j}}\right) m_{j}+m_{j+1}=\frac{3}{h_{j}}\left[\frac{h_{j} u_{j+1}}{h_{j+1}}+\left(\frac{h_{j+1}}{h_{j}}-\frac{h_{j}}{h_{j+1}}\right) u_{j}-\frac{h_{j+1}}{h_{j}} u_{j-1}\right]_{\text {(B) }}$.

Similarly, the spline relationship (3.17) for $T_{i, j}$ was deduced from (3.9). It gives the following truncation error for $T_{i, j}$ : 


$$
\begin{aligned}
T_{i, j}= & \frac{\partial^{4} u_{i, j}}{\partial x_{1}^{2} x_{2}^{2}}-\frac{k_{j+1}^{2}-k_{j} k_{i+1}+k^{2}}{12} \frac{\partial^{6} u_{i, j}}{\partial x_{i}^{2} \partial x_{2}^{4}} \\
& -\frac{\left(k_{j+1}-k_{j}\right)\left(k_{j+1}+2 k_{j}\right)\left(2 k_{j+1}+k_{j}\right)}{180} \frac{\partial^{7} u_{i, j}}{\partial x_{1}^{2} \partial x_{2}^{5}} \\
& +\frac{\left(h_{i+1}-h_{i}\right)\left(h_{i+1}-3 h_{i}\right)\left(3 h_{i+1}-h_{i}\right)}{180} \frac{\partial^{7} u_{i, j}}{\partial x_{1}^{5} \partial x_{2}^{2}} \\
& +\frac{7 k_{j+1}^{4}-16 k_{j} k_{j+1}^{3}+21 k_{j}^{2} k_{j+1}^{2}-16 k_{j}^{3} k_{j+1}+7 k_{j}^{4}}{1080} \frac{\partial^{8} u_{i, j}}{\partial x_{i}^{2} \partial x_{2}^{6}} \\
& +\frac{7 h_{i+1}^{4}-16 h_{i} h_{i+1}^{3}+2 i h_{i}^{2} h_{i+1}^{2}-16 h_{i}^{3} h_{i+1}+7 h_{i}^{4}}{1080} \frac{\partial^{8} u_{i, j}}{\partial x_{i}^{6} \partial x_{2}^{2}} \\
& +O\left(h^{5}, k^{5}\right) .
\end{aligned}
$$

Then, using this result in (3.18), the Iruncation error for $\hat{T}_{1, j}$ can be found:

$$
\begin{aligned}
\widehat{T}_{i, j}= & \frac{\partial^{4} u_{i, j}}{\partial x_{i}^{2} x_{2}^{2}}-\frac{\left(k_{j+1}-k_{j}\right)\left(k_{j+1}-3 k_{j}\right)\left(3 k_{j+1}-k_{j}\right)}{180} \frac{\partial^{7} u_{i, j}}{\partial x_{i}^{2} \partial x_{2}^{5}} \\
& +\frac{\left(h_{i+1}-h_{i}\right)\left(h_{i+1}-3 h_{i}\right)\left(3 h_{i+1}-h_{i}\right)}{180} \frac{\partial^{7} u_{i, j}}{\partial x_{1}^{5} \partial x_{2}^{2}} \\
& +\frac{7 k_{j+1}^{4}-16 k_{j} k_{j+1}^{3}+21 k_{j}^{2} k_{j+1}^{2}-16 k_{j}^{3} k_{j+1}+7 k_{j}^{4}}{1080} \frac{\partial^{8} u_{i, j}}{\partial x_{1}^{2} \partial x_{2}^{6}} \\
& +\frac{7 h_{i+1}^{4}-16 h_{i} h_{i+1}^{3}+21 h_{i}^{2} h_{i+1}^{2}-16 h_{i}^{3} h_{i+1}+7 h_{i}^{4}}{1080} \frac{\partial^{8} u_{i, j}}{\partial c_{i}^{6} \partial x_{2}^{2}} \\
& +\mathscr{O}\left(h^{5}, k^{5}\right) .
\end{aligned}
$$

Although this expression is not required in the developizent of the boundary 
conditions below, it is still of interest.

We can now proceed with the boundary conditions for the spline derivative coefficients. As was noted in Chapter 3 , because of the two one-sided expressions for $m_{j}$, no new boundary conditions are needed for $m$, or $m_{N}$. For second derivatives, there are two separate boundary conditions to compute. They are first, a means of computing $K_{1}$ and $K_{N}$ to as close to the truncation error of (B2) as possible, and second, an expression for the second derivative of $u$ which has the same order of accuracy as $G_{j}$ in (B4). The reason for these consistency requirements stems from the need of $K$, and $K_{N}$ in computing the spline tridiagonal relationship for $K_{i}$ to an accuracy which does not contaminate the calculation of the $K_{i}^{\prime} \mathrm{s}$. This holds true of $G$, and $G_{N}$ too, as they are required in the perhaps more numerically sensitive MHD equations.

By some trial and error, an expression for $K$, was found to be

$$
\begin{aligned}
K_{1} & +\frac{2 h_{3}^{2}+11 h_{2} h_{3}+11 h_{2}^{2}}{6 h_{2}^{2}} K_{2}+\frac{h_{3}^{2}+4 h_{2} h_{3}+h_{2}^{2}}{6 h_{2}^{2}} K_{3} \\
& -\frac{\left(h_{3}+2 h_{2}\right)\left(h_{3}+3 h_{2}\right)}{h_{2}^{3}\left(h_{3}+h_{2}\right)} u_{1}+\frac{\left(h_{3}+2 h_{2}\right)\left(h_{3}+3 h_{2}\right)}{h_{2}^{3} h_{3}} u_{2}-\frac{\left(h_{3}+2 h_{2}\right)\left(h_{3}+3 h_{2}\right)}{h_{2}^{2} h_{3}\left(h_{3}+h_{2}\right)} u_{3}=0,
\end{aligned}
$$

with implied truncation error:

$$
K_{1}=\frac{\partial^{2} u_{1}}{\partial x^{2}}-\frac{h_{2}^{2}}{12} \frac{\partial^{4} u_{1}}{\partial x^{4}}+\frac{\left(h_{3}-h_{2}\right)\left(h_{3}+2 h_{2}\right)\left(3 h_{3}-h_{2}\right)}{180} \frac{\partial^{5} u_{1}}{\partial x^{5}}+\mathscr{O}\left(h^{4}\right) .
$$


It is of note that this truncation error is nearly the same as (B2), and in fact, they are identical on a uniform mesh. While this relation is very attractive, there are other possible relations for $K_{f}$. One is:

$$
K_{1}-\frac{2 h_{2}+h_{3}}{h_{3}-h_{2}} K_{2}+\frac{6}{\left(h_{3}-h_{2}\right)\left(h_{3}+h_{2}\right)} u_{1}-\frac{6}{h_{3}\left(h_{3}-h_{2}\right)} u_{2}+\frac{6 h_{2}}{h_{3}\left(h_{3}-h_{2}\right)\left(h_{3}+h_{2}\right)} u_{3}=0 .
$$

However, because this second-order relation for $K$, is singular on a uniform mesh, it is rejected. The other possible second-order relation is an extrapolation in terms of $K^{\prime} s$ only:

$$
K_{1}-\frac{h_{2}+h_{3}}{h_{3}} K_{2}+\frac{h_{2}}{h_{3}} K_{3}=0
$$

The truncation error in this case is

$$
\begin{aligned}
K_{1}= & \frac{\partial^{2} u_{1}}{\partial x^{2}}-\frac{7 h_{2}^{2}+5 h_{2} h_{3}+h_{3}^{2}}{12} \frac{\partial^{4} u_{1}}{\partial x^{4}}-\frac{\left(2 h_{2}+h_{3}\right)\left(29 h_{2}\left(h_{2}+h_{3}\right)+2 h_{3}^{2}\right)}{180} \frac{\partial^{5} u_{1}}{\partial x^{5}} \\
& +\frac{7 h_{3}^{4}-16 h_{2} h_{3}^{3}-159 h_{2}^{2} h_{3}^{2}-286 h_{2}^{3} h_{3}-83 h_{2}^{4}}{1080} \frac{\partial^{6} u_{1}}{\partial x^{6}} \\
& +\mathscr{O}\left(h^{5}\right)
\end{aligned}
$$

which is slightly less accurate than (B2) and (B10). This last method is a very attractive one, particularly if it is desired to compute the $K^{\prime \prime}$ s separately from the plasma calculation as discussed in Chapter 4. However, pure extrapolation 
techniques are notorious for numerical difficulties they can introduce. Nonetheless, at second-order, the extrapolation problems should be minimized. The fact that relation (B10) is not strictly an extrapolation argues in its favor, and we therefore opt to use it.

At the other boundary, a similar set of relations can be found for $K_{N}$. Only the one corresponding to $(\mathrm{B} 10)$ is given here. It is

$$
\begin{aligned}
K_{N} & +\frac{2 h_{N-1}^{2}+11 h_{N}{ }^{2}{ }_{N-1}+11 h_{N}^{2}}{6 h_{N}^{2}} K_{N-1}+\frac{h_{N-1}^{2}+4 h_{N} h_{N-1}+h_{N}^{2}}{6 h_{N}^{2}} K_{N-2} \\
& -\frac{\left(h_{N-1}+2 h_{N}\right)\left(h_{N-1}+3 h_{N}\right)}{h_{N}^{3}\left(h_{N-1}+h_{N}\right)} u_{N}+\frac{\left(h_{N-1}+2 h_{N}\right)\left(h_{N-1}+3 h_{N}\right)}{h_{N}^{3} h_{N-1}} u_{N-1} \\
& -\frac{\left(h_{N-1}+2 h_{N}\right)\left(h_{N-1}+3 h_{N}\right)}{h_{N}^{2} h_{N-1}\left(h_{N-1}+h_{N}\right)} u_{N-2}=0 .
\end{aligned}
$$

with truncation error given by

$$
K_{N}=\frac{\partial^{2} u_{N}}{\partial x^{2}}-\frac{h_{N}^{2}}{12} \frac{\partial^{4} u_{N}}{\partial x^{4}}-\frac{\left(h_{N-1}-h_{N}\right)\left(h_{N-1}+2 h_{N}\right)\left(3 h_{N-1}-h_{N}\right)}{180} \frac{\partial^{5} u_{N}}{\partial x^{5}}+\mathscr{O}\left(h^{4}\right)
$$

For the approximation of $G_{1}$, there are again several possibilities. The first we considered was of the general form of (B10), with a non-unity coefficient multiplying $K$, and $G$, replacing the zero on the right-hand side. Unfortunately, it was singular on a uniform mesh, and was therefore discarded. The second was another three-point scheme that is a one-sided approximation: 


$$
G,=\frac{h_{3}^{2}+5 h_{2} h_{3}+7 h_{2}^{2}}{\left(h_{3}+2 h_{2}\right)\left(h_{3}+3 h_{2}\right)} K_{1}-\frac{h_{2}^{2}\left(h_{2}+h_{3}\right)}{h_{3}\left(h_{3}+2 h_{2}\right)\left(h_{3}+3 h_{2}\right)} K_{2}+\frac{h_{2}^{3}}{h_{3}\left(h_{3}+2 h_{2}\right)\left(h_{3}+3 h_{2}\right)} K_{3},
$$

with truncation error equal to

$$
G_{1}=\frac{\partial^{2} u_{1}}{\partial x^{2}}+\frac{\left(h_{3}+2 h_{2}\right)\left(3 h_{3}^{2}-4 h_{2} h_{3}+6 h_{2}^{2}\right)}{180} \frac{\partial^{5} u_{1}}{\partial x^{5}}+\mathcal{O}\left(h^{4}\right)
$$

The last form tried was a four-point one-sided approximation to improve the accuracy of $G_{1}$. With the preliminary definitions

$$
\begin{aligned}
& d_{1}=30 h_{2}^{2} h_{3} h_{4}^{2}+30 h_{2}^{3} h_{4}^{2}+18 h_{3}^{4} h_{4}+30 h_{2} h_{3}^{3} h_{4}+60 h_{2}^{2} h_{3}^{2} h_{4}+150 h_{2}^{3} h_{3} h_{4} \\
& d_{2}=102 h_{2}^{4} h_{4}+21 h_{3}^{5}+47 h_{2} h_{3}^{4}+56 h_{2}^{2} h_{3}^{3}+134 h_{2}^{3} h_{3}^{2}+150 h_{2}^{4} h_{3}+72 h_{2}^{5} \\
& d_{3}=30 h_{2}^{3} h_{4}^{2}+18 h_{2} h_{3}^{3} h_{4}+12 h_{2}^{2} h_{3}^{2} h_{4}+18 h_{2}^{3} h_{3} h_{4}+102 h_{2}^{4} h_{4}+3 h_{3}^{5} \\
& d_{4}=17 h_{2} h_{3}^{4}+26 h_{2}^{2} h_{3}^{3}+14 h_{2}^{3} h_{3}^{2}+48 h_{2}^{4} h_{3}+72 h_{2}^{5} .
\end{aligned}
$$

the new one-sided form was 


$$
\begin{aligned}
G_{1}= & \frac{5 h_{3}^{2} h_{4}+25 h_{2} h_{3} h_{4}+35 h_{2}^{2} h_{4}+8 h_{3}^{3}+32 h_{2} h_{3}^{2}+58 h_{2}^{2} h_{3}+47 h_{2}^{3}}{5\left(h_{3}+2 h_{2}\right)\left(h_{3}+3 h_{2}\right)\left(h_{2}+h_{3}+h_{4}\right)} K_{1} \\
& -\frac{d_{1}+d_{2}}{30 h_{3}\left(h_{3}+h_{4}\right)\left(h_{3}+2 h_{2}\right)\left(h_{3}+3 h_{2}\right)\left(h_{2}+h_{3}+h_{4}\right)} K_{2} \\
& +\frac{d_{3}+d_{4}}{30 h_{3} h_{4}\left(h_{3}+2 h_{2}\right)\left(h_{3}+3 h_{2}\right)\left(h_{2}+h_{3}+h_{4}\right)} K_{3} \\
& -\frac{\left(h_{3}+2 h_{2}\right)\left(3 h_{3}^{2}-4 h_{2} h_{3}+6 h_{2}^{2}\right)}{30 h_{4}\left(h_{3}+h_{4}\right)\left(h_{2}+h_{3}+h_{4}\right)} K_{4} .
\end{aligned}
$$

The truncation error implied by this equation is

$$
\begin{aligned}
G_{1}= & \frac{\partial^{2} u_{1}}{\partial x^{2}} \\
& -\frac{9 h_{3}^{3} h_{4}+6 h_{2} h_{3}^{2} h_{4}-6 h_{2}^{2} h_{3} h_{4}+36 h_{2}^{3} h_{4}+4 h_{3}^{4}+11 h_{2} h_{3}^{3}-6 h_{2}^{2} h_{3}^{2}+56 h_{2}^{3} h_{3}+64 h_{2}^{4}}{2160} \frac{\partial^{6} u,}{\partial x^{6}}+\mathscr{O}\left(h^{5}\right),
\end{aligned}
$$

which is of value if consistency with (B4) on a uniform mesh is desired. Note that the relations for $G_{N}$ are similar to those for $G$, and are not given here.

Because the mixed derivatives $\hat{l}_{i, j}$ are being treated explicitly in the plasma calculation, boundary relations for $\hat{l}$ and all higher-order spline derivative coefficients can be given in terms of any spline quantity developed thus far.

For $\hat{l}_{i, j}$, the solution process described in Chapter 4 goes along constant $x_{1}$ lines. Consequently, conditions at $\left(x_{2}\right)$, and $\left(x_{2}\right)_{N}$ are required: $\hat{l}_{i, f}$ and $\hat{l}_{i, N}$. For the first, we have 


$$
\begin{aligned}
\hat{l}_{i, 1} & +\frac{\left(k_{2}+k_{3}\right)\left(3 k_{3}^{2}+5 k_{2} k_{3}+4 k_{2}^{2}\right)}{2 k_{3}\left(k_{3}^{2}+k_{2} k_{3}+k_{2}^{2}\right)} \hat{l}_{i, 2}+\frac{k_{2}\left(k_{2}+k_{3}\right)\left(k_{3}+2 k_{2}\right)}{2 k_{3}\left(k_{3}^{2}+k_{2} k_{3}+k_{2}^{2}\right)} \hat{\imath}_{i, 3} \\
& +\frac{5 k_{3}^{3}+13 k_{2} k_{3}^{2}+12 k_{2}^{2} k_{3}+6 k_{2}^{3}}{2 k_{2}\left(k_{2}+k_{3}\right)\left(k_{3}^{2}+k_{2} k_{3}+k_{2}^{2}\right)} m_{i, 1}-\frac{\left(k_{3}-k_{2}\right)\left(k_{3}+k_{2}\right)\left(5 k_{3}^{2}+8 k_{2} k_{3}+6 k_{2}^{2}\right)}{2 k_{2} k_{3}^{2}\left(k_{3}^{2}+k_{2} k_{3}+k_{2}^{2}\right)} m_{2,2} \\
& -\frac{k_{2}\left(k_{3}+3 k_{2}\right)\left(3 k_{3}^{2}+4 k_{2} k_{3}+2 k_{2}^{2}\right)}{2 k_{3}^{2}\left(k_{2}+k_{3}\right)\left(k_{3}^{2}+k_{2} k_{3}+k_{2}^{2}\right)} m_{i, 3}=0 .
\end{aligned}
$$

with truncation error

$$
\begin{aligned}
\hat{l}_{i, 1}= & \frac{\partial^{2} u_{i, 1}}{\partial x_{1} \partial x_{2}}-\frac{h_{i} h_{i+1}\left(h_{i+1}-h_{i}\right)}{24} \frac{\partial^{5} u_{i, 1}}{\partial x_{1}^{4} \partial x_{2}} \\
& -\frac{h_{i} h_{i+1}\left(h_{i+1}^{2}-h_{i} h_{i+1}+h_{i}^{2}\right)}{180} \frac{\partial^{6} u_{i, 1}}{\partial x_{1}^{5} \partial x_{2}}+\frac{k_{2}^{2} k_{3}\left(k_{2}+k_{3}\right)\left(k_{3}^{2}+k_{2} k_{3}-5 k_{2}^{2}\right)}{360\left(k_{3}^{2}+k_{2} k_{3}+k_{2}^{2}\right)} \frac{\partial^{6} u_{i, 1}}{\partial x_{1} \partial x_{2}^{5}} \\
& +\mathcal{O}\left(h^{5}, k^{5}\right) .
\end{aligned}
$$

The condition at the other boundary is

$$
\begin{aligned}
& \hat{\iota}_{i, M}+\frac{\left(k_{N}+k_{M-1}\right)\left(3 k_{M-1}^{2}+5 k_{N} k_{M-1}+4 k_{N}^{2}\right)}{2 k_{M-1}\left(k_{M-1}^{2}+k_{N} k_{M-1}+k_{N}^{2}\right)} \hat{\iota}_{i, M-1}+\frac{k_{M}\left(k_{M}+k_{N-1}\right)\left(k_{N-1}+2 k_{N}\right)}{2 k_{M-1}\left(k_{M-1}^{2}+k_{N} k_{M-1}+k_{M}^{2}\right)} \hat{\iota}_{2, M-2} \\
& -\frac{5 k_{M-1}^{3}+13 k_{N} k_{M-1}^{2}+12 k_{N}^{2} k_{N-1}+6 k_{N}^{3}}{2 k_{M}\left(k_{M}+k_{N-1}\right)\left(k_{M-1}^{2}+k_{N} k_{N-1}+k_{N}^{2}\right)} m_{i, H} \\
& +\frac{\left(k_{M-1}-k_{N}\right)\left(k_{N-1}+k_{N}\right)\left(5 k_{M-1}^{2}+8 k_{N} k_{M-1}+6 k_{M}^{2}\right)}{2 k_{N} k_{M-1}^{2}\left(k_{M-1}^{2}+k_{N} k_{N-1}+k_{N}^{2}\right)} m_{i, M-1} \\
& +\frac{k_{M}\left(k_{M-1}+3 k_{M}\right)\left(3 k_{M-1}^{2}+4 k_{M} k_{N-1}+2 k_{M}^{2}\right)}{2 k_{M-1}^{2}\left(k_{M}+k_{N-1}\right)\left(k_{M-1}^{2}+k_{N} k_{N-1}+k_{M}^{2}\right)} m_{i, N-2}=0 \text {. }
\end{aligned}
$$

with truncation error 


$$
\begin{aligned}
\hat{l}_{i, M}= & \frac{\partial^{2} u_{i, M}}{\partial x_{1} \partial x_{2}}-\frac{h_{i} h_{i+1}\left(h_{i+1}-h_{i}\right)}{24} \frac{\partial^{5} u_{i, M}}{\partial x_{1}^{4} \partial x_{2}}-\frac{h_{i} h_{i+1}\left(h_{i+1}^{2}-h_{i} h_{i+1}+h_{i}^{2}\right)}{180} \frac{\partial^{6} u_{i, M}}{\partial x_{1}^{5} \partial x_{2}} \\
& +\frac{k_{M}^{2} k_{M-1}\left(k_{M}+k_{M-1}\right)\left(k_{M-1}^{2}+k_{M} k_{M-1}-5 k_{M}^{2}\right)}{360\left(k_{M-1}^{2}+k_{M} k_{M-1}+k_{M}^{2}\right)} \frac{\partial^{6} u_{i, M}}{\partial x_{1} \partial x_{2}^{5}} \\
& +\mathcal{O}\left(h^{5}, k^{5}\right) .
\end{aligned}
$$

Proceeding now to the boundary conditions on $\widehat{K}_{i, j}$, since this variable is found by solving a set of tridiagonal relationships in the $x_{z}$-direction, the left $x_{2}$ boundary relation was found to be:

$$
\begin{aligned}
\hat{K}_{i, 1} & +\frac{\left(k_{2}+k_{3}\right)\left(3 k_{3}^{2}+5 k_{2} k_{3}+4 k_{2}^{2}\right)}{2 k_{3}\left(k_{3}^{2}+k_{2} k_{3}+k_{2}^{2}\right)} \hat{K}_{i, 2}+\frac{k_{2}\left(k_{2}+k_{3}\right)\left(k_{3}+2 k_{2}\right)}{2 k_{3}\left(k_{3}^{2}+k_{2} k_{3}+k_{2}^{2}\right)} \hat{K}_{i, 3} \\
& +\frac{5 k_{3}^{3}+13 k_{2} k_{3}^{2}+12 k_{2}^{2} k_{3}+6 k_{2}^{3}}{2 k_{2}\left(k_{2}+k_{3}\right)\left(k_{3}^{2}+k_{2} k_{3}+k_{2}^{2}\right)} G_{i, 1}-\frac{\left(k_{3}-k_{2}\right)\left(k_{3}+k_{2}\right)\left(5 k_{3}^{2}+8 k_{2} k_{3}+6 k_{2}^{2}\right)}{2 k_{2} k_{3}^{2}\left(k_{3}^{2}+k_{2} k_{3}+k_{2}^{2}\right)} G_{i, 2} \\
& -\frac{k_{2}\left(k_{3}+3 k_{2}\right)\left(3 k_{3}^{2}+4 k_{2} k_{3}+2 k_{2}^{2}\right)}{2 k_{3}^{2}\left(k_{2}+k_{3}\right)\left(k_{3}^{2}+k_{2} k_{3}+k_{2}^{2}\right)} G_{i, 3}=0 .
\end{aligned}
$$

The truncation error for $\widehat{K}_{i, f}$ is found from this relation to be

$$
\begin{aligned}
\hat{K}_{i, 1}= & \frac{\partial^{3} u_{i, 1}}{\partial x_{1}^{2} \partial x_{2}}+\frac{\left(h_{i+1}-h_{i}\right)\left(h_{i+1}-3 h_{i}\right)\left(3 h_{i+1}-h_{i}\right)}{180} \frac{\partial^{6} u_{i, 1}}{\partial x_{1}^{5} \partial x_{2}} \\
& +\frac{7 h_{i+1}^{4}-16 h_{i} h_{i+1}^{3}+21 h_{i}^{2} h_{i+1}^{2}-16 h_{i}^{3} h_{i+1}+7 h_{i}^{4}}{1080} \frac{\partial^{7} u_{i, 1}}{\partial x_{1}^{6} \partial x_{2}} \\
& +\frac{k_{2}^{2} k_{3}\left(k_{2}+k_{3}\right)\left(k_{3}^{2}+k_{2} k_{3}-5 k_{2}^{2}\right)}{360\left(k_{3}^{2}+k_{2} k_{3}+k_{2}^{2}\right)} \frac{\partial^{7} u_{i, 1}}{\partial x_{1}^{2} \partial x_{2}^{5}} \\
& +\mathcal{O}\left(h^{5}, k^{5}\right) .
\end{aligned}
$$


At the right $x_{2}$ boundary, we find

$$
\begin{aligned}
\hat{K}_{2, M} & +\frac{\left(k_{M}+k_{M-1}\right)\left(3 k_{M-1}^{2}+5 k_{M} k_{M-1}+4 k_{M}^{2}\right)}{2 k_{M-1}\left(k_{M-1}^{2}+k_{M} k_{M-1}+k_{M}^{2}\right)} \hat{K}_{i, M-1}+\frac{k_{M}\left(k_{M}+k_{M-1}\right)\left(k_{M-1}+2 k_{M}\right)}{2 k_{M-1}\left(k_{M-1}^{2}+k_{M} k_{M-1}+k_{M}^{2}\right)} \hat{K}_{2, M-2} \\
& -\frac{5 k_{M-1}^{3}+13 k_{M} k_{M-1}^{2}+12 k_{M}^{2} k_{M-1}+6 k_{M}^{3}}{2 k_{M}\left(k_{M}+k_{M-1}\right)\left(k_{M-1}^{2}+k_{M} k_{M-1}+k_{M}^{2}\right)} G_{i, M} \\
& +\frac{\left(k_{M-1}-k_{M}\right)\left(k_{M-1}+k_{M}\right)\left(5 k_{M-1}^{2}+8 k_{M} k_{N-1}+6 k_{M}^{2}\right)}{2 k_{M} k_{M-1}^{2}\left(k_{M-1}^{2}+k_{M} k_{M-1}+k_{M}^{2}\right)} G_{i, M-1} \\
& +\frac{k_{M}\left(k_{M-1}+3 k_{M}\right)\left(3 k_{M-1}^{2}+4 k_{M} k_{M-1}+2 k_{M}^{2}\right)}{2 k_{M-1}^{2}\left(k_{M}+k_{M-1}\right)\left(k_{M-1}^{2}+k_{M} k_{M-1}+k_{M}^{2}\right)} G_{1, M-2}=0,
\end{aligned}
$$

with associated truncation error

$$
\begin{aligned}
\hat{K}_{i, M}= & \frac{\partial^{3} u_{i, N}}{\partial x_{1}^{2} \partial x_{2}}+\frac{\left(h_{i+1}-h_{i}\right)\left(h_{i+1}-3 h_{i}\right)\left(3 h_{2+1}-h_{i}\right)}{180} \frac{\partial^{6} u_{2, M}}{\partial x_{1}^{5} \partial x_{2}} \\
& +\frac{7 h_{i+1}^{4}-16 h_{i} h_{i+1}^{3}+21 h_{i}^{2} h_{i+1}^{2}-16 h_{2}^{3} h_{i+1}+7 h_{i}^{4}}{1080} \frac{\partial^{7} u_{2, M}}{\partial x_{1}^{6} \partial x_{2}} \\
& +\frac{k_{M}^{2} k_{M-1}\left(k_{N}+k_{N-1}\right)\left(k_{M-1}^{2}+k_{N} k_{N-1}-5 k_{M}^{2}\right)}{360\left(k_{N-1}^{2}+k_{N} k_{N-1}+k_{N}^{2}\right)} \frac{\partial^{7} u_{i, M}}{\partial x_{1}^{2} \partial x_{2}^{5}} \\
& +O\left(h^{5}, k^{5}\right) .
\end{aligned}
$$

Because of the symmetry between $\hat{L}$ and $\hat{K}$ discussed earlier. no boundary conditions need be given here for $\tilde{L}$.

The last variable to be solved by a tridiagonal relation is $T_{i, j}$. At the left $x_{2}$ boundary, it was found that 


$$
\begin{aligned}
T_{i, 1} & +\frac{2 k_{3}^{2}+11 k_{2} k_{3}+11 k_{2}^{2}}{6 k_{2}^{2}} T_{i, 2}+\frac{k_{3}^{2}+4 k_{2} k_{3}+k_{2}^{2}}{6 k_{2}^{2}} T_{i, 3} \\
& -\frac{\left(k_{3}+2 k_{2}\right)\left(k_{3}+3 k_{2}\right)}{k_{2}^{3}\left(k_{3}+k_{2}\right)} G_{i, 1}+\frac{\left(k_{3}+2 k_{2}\right)\left(k_{3}+3 k_{2}\right)}{k_{2}^{3} k_{3}} G_{i, 2}-\frac{\left(k_{3}+2 k_{2}\right)\left(k_{3}+3 k_{2}\right)}{k_{2}^{2} k_{3}\left(k_{3}+k_{2}\right)} G_{i, 3}=0 .
\end{aligned}
$$

$T_{i, 1}$ 's truncation error is then computed via Taylor expansion of the other quantities, and is found to be

$$
\begin{aligned}
T_{i, 1}= & \frac{\partial^{4} u_{i, 1}}{\partial x_{1}^{2} \partial x_{2}^{2}}-\frac{k_{2}^{2}}{12} \frac{\partial^{6} u_{i, 1}}{\partial x_{1}^{2} \partial x_{2}^{4}} \\
& +\frac{\left(h_{i+1}-h_{i}\right)\left(h_{i+1}-3 h_{i}\right)\left(3 h_{i+1}-h_{i}\right)}{180} \frac{\partial^{7} u_{i, 1}}{\partial x_{1}^{5} \partial x_{2}^{2}}+\frac{\left(k_{3}-k_{2}\right)\left(k_{3}+2 k_{2}\right)\left(3 k_{3}-k_{2}\right)}{180} \frac{\partial^{7} u_{i, 1}}{\partial x_{t}^{2} \partial x_{2}^{5}} \\
& +\frac{7 h_{i+1}^{4}-16 h_{i} h_{i+1}^{3}+21 h_{i}^{2} h_{i+1}^{2}-16 h_{i}^{3} h_{i+1}+7 h_{i}^{4}}{1080} \frac{\partial^{8} u_{i, 1}}{\partial x_{1}^{6} \partial x_{2}^{2}} \\
& +\frac{7 k_{3}^{4}+14 k_{2} k_{3}^{3}+6 k_{2}^{2} k_{3}^{2}-31 k_{2}^{2} k_{3}+7 k_{2}^{4}}{1080} \frac{\partial^{8} u_{i, 1}}{\partial x_{1}^{2} \partial x_{2}^{6}} \\
& +0\left(h^{5}, k^{5}\right) .
\end{aligned}
$$

The relation for $T_{i, N}$ 's is similar and is therefore not given. It remains to give the truncation error for $\widehat{T}_{i, j}$ at the boundaries. If the relation for $\hat{T}_{2,1}$ is chosen analogously to $(\mathrm{B} 18)$, i.e..

$$
\begin{aligned}
\widehat{T}_{i, 1}= & \frac{k_{3}^{2}+5 k_{2} k_{3}+7 k_{2}^{2}}{\left(k_{3}+2 k_{2}\right)\left(k_{3}+3 k_{2}\right)} T_{i, 1}-\frac{k_{2}^{2}\left(k_{2}+k_{3}\right)}{k_{3}\left(k_{3}+2 k_{2}\right)\left(k_{3}+3 k_{2}\right)} T_{i, 2} \\
& +\frac{k_{2}^{3}}{k_{3}\left(k_{3}+2 k_{2}\right)\left(k_{3}+3 k_{2}\right)} T_{i, 3} .
\end{aligned}
$$


then the truncation error for $\widehat{T}_{2,}$, will be analogous to (B19). It is

$$
\begin{aligned}
\widehat{T}_{1,1}= & \frac{\partial^{4} u_{1,1}}{\partial x_{1}^{2} x_{2}^{2}} \\
& +\frac{\left(k_{3}+2 k_{2}\right)\left(3 k_{3}^{2}-4 k_{2} k_{3}+6 k_{2}^{2}\right)}{180} \frac{\partial^{7} u_{i, 1}}{\partial x_{1}^{2} \partial x_{2}^{5}}+\frac{\left(h_{i+1}-h_{i}\right)\left(h_{i+1}-3 h_{2}\right)\left(3 h_{i+1}-h_{2}\right)}{180} \frac{\partial^{7} u_{1,1}}{\partial x_{1}^{5} \partial x_{2}^{2}} \\
& +\mathscr{C}\left(h^{4}, k^{4}\right) .
\end{aligned}
$$

Once again, analogous relations apply at the other boundary for $\hat{T}_{1, N}$. Fourth-order relations are possible in each case. 


\section{APPENDIX C}

\section{BI-QUINTIC INTERPOLANT}

With the boundary information determined by relations developed in Appendix $B$ together with the tridiagonal relations given in Chapter 3 , there are 8 spline derivative coefficients and the functional value known at each grid point: $m_{i, j}, l_{i, j}, G_{i, j}, H_{i, j}, \hat{l}_{i, j}, \hat{K}_{i, j}, \hat{L}_{i, j}, \hat{T}_{i, j}$, and $u_{i, j}$. This completely determines the bi-quintic spline interpolant, but the relationship between these spline derivative coefficients and the coefficients of the interpolant is non-trivial; it involves a linear transformation. First, we give the bi-quintic interpolating polynomial:

$$
\mathcal{F}\left(x_{1}, x_{2}\right)=\sum_{m=0}^{5} \sum_{n=0}^{5} a_{m n}\left(\frac{x_{1}-x_{i-1}, j}{h_{i}}\right)^{m}\left(\frac{x_{2}-x_{i, j-1}}{k_{j}}\right)^{n} .
$$

Sow the relationship can be detailed at each of the four corner points telimiting the rectangle over which each bi-quintic interpolant is defined. At $\because$ is : ower left corner, that is, at $x=x_{i-1, j-1}$, these relations are 


$$
\begin{aligned}
& u_{2-1, j-1}=\left.\mathscr{f}\right|_{1-1,1,1}=a_{00} \quad \quad \hat{l}_{2-1, j-1}=\left.\frac{\partial^{2} \mathscr{y}}{\partial x, \partial x_{2}}\right|_{2-1, j-1}=\frac{a_{11}}{h_{2} k} \\
& m_{2-1,3-1}=\left.\frac{\partial y}{\partial x}\right|_{2-1,3-1}=\frac{a_{10}}{h_{2}} \quad l_{i-1, j-i}=\left.\frac{\partial y}{\partial x_{2}}\right|_{2-1,3-1}=\frac{a_{01}}{k_{3}} \\
& G_{2-1, j-1}=\left.\frac{\partial^{2} y}{\partial x_{1}^{2}}\right|_{2-1, j-1}=\frac{2 a_{20}}{h_{i}^{2}} \quad H_{2-1, j-1}=\left.\frac{\partial^{2} y}{\partial x_{2}^{2}}\right|_{i-1, j-1}=\frac{2 a_{02}}{k_{j}^{2}} \\
& \widehat{K}_{2-1, j-1}=\left.\frac{\partial^{3} \mathscr{y}}{\partial x_{1}^{2} \partial x_{2}}\right|_{i-1, j-1}=\frac{2 a_{21}}{h_{2}^{2} k} \quad \hat{L}_{i-1, j-1}=\left.\frac{\partial^{3} \mathscr{y}}{\partial x_{1} \partial x_{2}^{2}}\right|_{i-1, j-1}=\frac{2 a_{12}}{h_{2} k_{j}^{2}} \\
& \widehat{T}_{i-1, j-1}=\left.\frac{\partial^{4} \mathscr{S}}{\partial x_{1}^{2} \partial x_{2}^{2}}\right|_{i-1, j-1}=\frac{4 a_{22}}{h_{i}^{2} k_{j}^{2}}
\end{aligned}
$$

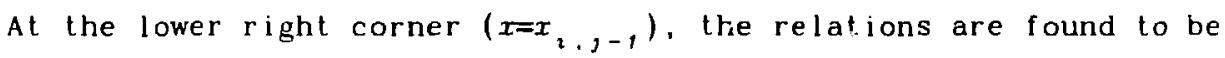

$$
\begin{array}{rlrl}
u_{i, j-1}=\sum_{m=0}^{5} a_{m} 0 & \hat{l}_{i, j-1} & =\frac{1}{h_{i} k_{j}} \sum_{m=1}^{5} m a_{m} \\
m_{i, j-1}=\frac{1}{h_{i}} \sum_{m=1}^{5} m a_{m 0} & l_{i, j-1}=\frac{1}{k} \sum_{m=0}^{5} a_{m}, \\
G_{i, j-1}=\frac{1}{h_{i}^{2}} \sum_{m=2}^{5} m(m-1) a_{m} 0 & H_{i, j-1}=\frac{2}{k_{j}^{2}} \sum_{m=0}^{5} a_{m 2} \\
\hat{K}_{i, j-1}=\frac{1}{h_{i}^{2} k} \sum_{m=2}^{5} m(m-1) a_{m}, & \hat{L}_{i, j-1}=\frac{2}{h_{i} k_{j}^{2}} \sum_{m=1}^{5} m a_{m 2} \\
\hat{T}_{i, j-1}=\frac{2}{h_{i}^{2} k_{j}^{2}} \sum_{m=2}^{5} m(m-1) a_{m 2}
\end{array}
$$

At the upper left corner $\left(x=x_{i-1, j}\right)$, the expressions become 


$$
\begin{array}{rlrl}
u_{i-1, j}=\sum_{n=0}^{5} a_{0_{n}} & \hat{l}_{i-1, j}=\frac{1}{h_{i} k} \sum_{n=1}^{5} n a_{1 n} \\
m_{i-1, j}=\frac{1}{h_{i}} \sum_{n=0}^{5} a_{1 n} & l_{i-1, j}=\frac{1}{k_{j}} \sum_{n=1}^{5} n a_{1 n} \\
G_{i-1, j}=\frac{2}{h_{i}^{2}} \sum_{n=0}^{5} a_{2 n} & H_{i-1, j}=\frac{1}{k_{j}^{2}} \sum_{n=2}^{5} n(n-1) a_{0 n} \\
\widehat{K}_{i-1, j}=\frac{2}{h_{i}^{2} k} \sum_{j=1}^{5} n a_{2 n} & \hat{L}_{i-1, j}=\frac{1}{h_{i} k_{j}^{2}} \sum_{n=2}^{5} n(n-1) a_{1 n} \\
\hat{T}_{i-1, j}=\frac{2}{n_{i}^{2} k_{j}^{2}} \sum_{n=2}^{5} n(n-1) a_{2 n}
\end{array}
$$

Finally, there remains the expressions for the spline derivative coefficients at the upper right corner $\left(x=x_{i, j}\right)$.

$$
\begin{array}{rlrl}
u_{i, j}=\sum_{m=0}^{5} \sum_{n=0}^{5} a_{m n} & \hat{l}_{i, j} & =\frac{1}{h_{i} k} \sum_{m=1}^{5} \sum_{n=1}^{5} m \pi a_{m n} \\
m_{i, j}= & -\frac{1}{h_{i}} \sum_{m=1}^{5} \sum_{n=0}^{5} m a_{m n} & \iota_{i, j} & =\frac{1}{k_{j}} \sum_{m=0}^{5} \sum_{n=1}^{5} n a_{m n} \\
G_{i, j}= & \frac{1}{h_{i}^{2}} \sum_{m=2}^{5} \sum_{n=0}^{5} m(m-1) a_{m n} & H_{i, j} & =\frac{1}{k_{j}^{2}} \sum_{m=0}^{5} \sum_{n=2}^{5} n(n-1) a_{m n} \\
\hat{K}_{i, j}= & \frac{1}{h_{i}^{2} k} \sum_{m=2}^{5} \sum_{n=1}^{5} m(m-1) m a_{m n} & \hat{L}_{i, j} & =\frac{1}{h_{i} k_{j}^{2}} \sum_{m=1}^{5} \sum_{n=2}^{5} m n(n-1) a_{m n} \\
\hat{T}_{i, j}=\frac{1}{h_{i}^{2} k_{j}^{2}} \sum_{m=2}^{5} \sum_{n=2}^{5} m(m-1)(n-1) a_{m n}
\end{array}
$$


These relations define 36 equations to determine the $a_{k}$ 's. Fortunately, 9 of them are known directly from the relations (C2), so that only 27 equations remain. An additional bonus is that the linear transformation between the interpolation coefficients $\left(a_{k}{ }^{\prime} s\right)$ and the spline derivative coefficients can be represented by an integer matrix. Therefore, an exact inversion of a $27 \times 27$ integer matrix is all that is required to obtain explicit expressions for the $a_{k}$ 's in terms of the spline derivative coefficients.

While there are some sparsity patterns in the $27 \times 27$ matrix which would make its inversion less difficult, there are more than adequate tools for dealing with an exact inversion problem. First of all, the inversion was performed via the congruence arithmetic methods in the ESOLVE package of Cabay and Lam, ${ }^{16}$ and a multiplication by the original matrix was performed to obtain the identity matrix as a check. As a second check, the inversion was performed in exact rational arithmetic in MACSYMA. Because the output for the interpolant was generated as highly opt imized FORTRAN code, it will not be given here. 


\section{APPENDIX D}

\section{NUMERICAL METHODS FOR THE}

\section{COMPLETE ELLIPTIC INTEGRALS $\boldsymbol{K}$ AND $\boldsymbol{E}$}

\section{D.1 Abstract}

The evaluation of $K$, the complete elliptic integral of the first kind, and of $E$, the complete elliptic integral of the second kind has been dealt with extensively, just as is the case for the entire field of elliptic functions. However, a comprehensive comparison of all of the numerical methods has never been done. This is done here for values of the modulus, $k$, in the range $0<k<1$. One new algorithm for $E$ was developed, and it compared favorably with the best methods near $k=1$. It was found that a hybrid algorithm using the theta nome expansion and a functional counterpart was optimal with regard to the number of terms used. On the other hand, the Hastings form method was slightly faster in vector execution speed. In terms of scalar operation, the two are roughly 
equivalent. If both $K$ and $E$ are needed, the theta nome hybrid method is super ior.

\section{D.2 Introduction}

The complete elliptic integrals $K$ and $E$ are given by

$$
\begin{aligned}
& K(k)=\int_{0}^{\pi / 2} \frac{d \varphi}{\sqrt{\left(1-k^{2} \sin ^{2} \varphi\right)}} \\
& E(k)=\int_{0}^{\pi / 2} \sqrt{ }\left(1-k^{2} \sin ^{2} \varphi\right) d \varphi .
\end{aligned}
$$

In this paper, we discuss the principal methods for computing $K$ and $E$. One of the methods for finding $E$ via an infinite product iterative technique is new. While there has been no attempt to present all possible formulas for performing these computations, the methods compared cover the classes of techniques presently known. The basis for comparison is two-fold. First, the actual computation speed achieved on the CRAY-1 in both scalar and vector modes for each computational method is compared. Then, the number of iterations required by the iterative methods or the number of terms necessary in methods using power series are contrasted. The actual amount of computer memory consumed by the vectorizing form of a given method is roughly proportional to its computation speed, so no comparison is made on the basis of memory size. 
In the next section, we introduce some notation important in the infinite product iterative methods, as well as some mappings which motivate the methods themselves. In section 3, the details of the methods for finding $K$ are given, including some methods which are immediately discarded. Of particular interest is a symmetry relation for the theta nome $q$ which motivates a functional relationship for finding $K$ near the singularity. Section 4 contains the details of the comparisons between these methods, where the measure of optimality includes both speed and fewest number of terms.

Turning our attention to $E$ in section 5 , the principal methods are once again presented. And just as the symmetry relation for $q$ allowed $u s$ to $f$ ind a functional relation for $K$, so too does it facilitaie the development of a functional relation for $E$ which is of value for $k \approx 1$. In section 6 , comparisons are made among all of the methods, but here there is a new twist to the analysis: some of the methods for finding $E$ require that $K$ already be known. So if the application in which evaluation of $K$ and/or $E$ arises calls for both values, then some of the conclusions change concerning the speed of computation. Another important observation in this section is that the arithmetic geometric mean formulation for finding $E$ has a built-in numerical instability close to $k=1$, and should therefore not be used in that region.

The last section, section 7 , summarizes our findings and makes recommendations concerning future implementations of complete elliptic integrators. Of particular interest is the fact that the lastings form method 
is the method of choice on current vector computers, while it is also the fastest means of finding $E$ alone in scalar mode. In terms of computing both $K$ and $E$ in scalar mode, the hybrid theta nome methods are found to be optimal. When the comparison criterion is fewest number of terms or iterations, the theta nome hybrid methods are superior for all values $k$.

\section{D.3 Infinite Product Approaches}

We wrile the iterates of a function $F$ under composition in a standard way:

$$
\begin{gathered}
F^{0}(k)=k \\
F^{m+1}(k)=F\left(F^{m}(k)\right), n \geqslant 0 .
\end{gathered}
$$

Several endomorphisms of the unit open interval (in $k$ ) are required. They are

$$
S: k \rightarrow k^{2} \quad, \quad T: k \rightarrow \frac{1-k^{\prime}}{1+k}, \quad R: k \rightarrow \frac{2 \sqrt{ } k}{1+k} .
$$

where the usual complementary modules definition holds $\left(k^{\prime}=\sqrt{ }\left(1-k^{2}\right)\right)$. In addition, define $L$ as follows

$$
L(k)=\frac{1-k^{2}}{2} K(k)
$$


Ferguson has shown that this leads to ${ }^{37}$

$$
L(k)=\frac{\pi}{4} \prod_{n=0}^{\infty} \frac{1-S\left(T^{n}(k)\right)}{1+T^{n+1}(k)}
$$

This result will be helpful later on in an algorithm for $\boldsymbol{E}$.

\section{D.4 Algorithms for $\boldsymbol{K}$}

The most popular algorithm is, of course, Gauss arithmetic-geometric mean. Starting with the two initial values $a_{0}=1$ and $b_{0}=k^{\circ}$, the iteration process can be written ${ }^{1}$

$$
\begin{aligned}
& a_{l}=\frac{1}{2}\left(a_{l-1}+b_{l-l}\right) \\
& b_{l}=V\left(a_{l-l} b_{l-1}\right) .
\end{aligned}
$$

It is terminated when $a_{N}=b_{N}$ to the required precision, and the $i$ inal value for $K$ is

$$
K(k)=\frac{\pi}{2 a_{N}} .
$$


A similar iterative procedure arises from the Landen transformation. ${ }^{15}$ The compact algorithm for the special case of $K$ is $^{14}$

$$
\begin{aligned}
& a_{l+1}=\frac{b_{l}}{\mu_{l}}+a_{l} \\
& b_{l+1}=2\left(\nu_{l} a_{l}+b_{l}\right) \\
& \mu_{l+1}=\mu_{l}+\nu_{l} \\
& \nu_{l+1}=2 \sqrt{ }\left(\mu_{l} \nu_{l}\right),
\end{aligned}
$$

where the initializations are given by

$$
a_{0}=b_{0}=\mu_{0}=1 \quad, \quad \nu_{0}=\left|k^{*}\right|
$$

Then,

$$
K \approx \frac{\pi}{4} \frac{a_{l}}{\mu_{l}}
$$

Yet a third iterative process is given by 900.02 in Byrd and Friedman. ${ }^{15}$ Using the notation introduced above, the iteration can be written ${ }^{36}$

$$
K=\frac{\pi}{2} \prod_{n=1}^{\infty}\left[1+T^{n}(k)\right]
$$

This infinite product would be terminated when $T^{m}(k)$ is zero to the required 
precision.

Because of the logarithmic singularity in $K$ at $k=1$, special forms have been developed to explicilly show the character of the singularity. Legendre form and a modified Legendre form are two such formulas; the modified Legendre form is $\mathrm{s}^{21}$

$$
K(k)=K_{1}\left(k^{\prime}\right)+K_{2}\left(k^{\prime}\right) \log \left(\frac{1}{\left(k^{\prime}\right)^{2}}\right)
$$

where

$$
\begin{aligned}
& K_{1}\left(k^{\prime}\right)=\log 4+\sum_{n=1}^{\infty}\left[\frac{(2 n-1) ! !}{2^{n} n !}\right]^{2}\left\{\log 4-2 \sum_{j=1}^{2 n} \frac{(-1)^{j-1}}{j}\right\}\left(k^{\prime}\right)^{2 n} \\
& K_{2}\left(k^{\prime}\right)=\frac{1}{2}\left\{1+\sum_{n=1}^{\infty}\left[\frac{(2 n-1) ! !}{2^{n} n !}\right]^{2}\left(k^{\prime}\right)^{2 n}\right\} .
\end{aligned}
$$

Because of the slow convergence characteristics of these power series, converting $K_{1}$ and $K_{2}$ to a series of Chebyshev polynomials has been attempted. ${ }^{22}$ Our experience with some of these has been that they are still slowly convergent, in part due to alternating signs in these series. Improvements were sought by Cody ${ }^{21}$ who found Chebyshev approximations of the Hastings form. For $K$, the Hastings form is 


$$
K(k) \approx \sum_{i=0}^{n} a_{i}\left(k^{\cdot}\right)^{2_{i}}+\log \left(\frac{1}{\left(k^{\prime}\right)^{2}}\right) \sum_{i=0}^{m} b_{i}\left(k^{\cdot}\right)^{2 i}
$$

with

$$
a_{0}=\log 4 . \quad \sum_{i=0}^{n} a_{i}=\frac{\pi}{2}, \quad b_{0}=\frac{1}{2}
$$

Cody also observed that the best numerical behavior was obtained when $n=m$. He gives the $a_{1}$ and $b_{2}$ for values of $n$ up to 10, and they are all positive. Hence. there will be monotonic convergence, as was indeed observed. Moreover, this method performed uniformly better than the series of Chebyshev polynomials.

Because power series can perform well in certain limited parameter regimes, we give two for $K$. First for $k<4$,

$$
K(k)=\frac{\pi}{2}\left\{1+\sum_{n=1}^{\infty}\left[\frac{(2 n-1) ! !}{2^{n} n !}\right]^{2} k^{2 n}\right\}=\pi K_{2}(k)
$$

In the singular region, $k \approx 1$, it is appropriate to use

$$
K\left(k^{\circ}\right)=\log \frac{4}{k^{\cdot}}+\sum_{n=1}^{\infty}\left[\frac{(2 n-1) ! !}{2^{n} n !}\right]^{2}\left\{\log \frac{4}{k^{\prime}}-2 \sum_{l=1}^{n} \frac{1}{(2 l-1) 2 l}\right\}\left(k^{\circ}\right)^{2 n}
$$

However, just as $K$, and $K_{2}$ ere slowly convergent over most of the parameter range, so will these series converge slowly outside the indicated regions. 
There remain, aside from variations on the techniques already discussed,

the theta function methods. A representation for $K$ in terms of theta functions is given by 1053.01 in Byrd and Friedman ${ }^{15}$ as

$$
K=\frac{\pi}{2}\left[\vartheta_{3}(0)\right]^{2}=\frac{\pi}{2}\left[1+2 \sum_{n=1}^{\infty} q^{n^{2}}\right]^{2}
$$

where the nome $q$ can be expressed as

$$
q=\sum_{n=0}^{\infty} \delta_{n} \varepsilon^{4 n+t}
$$

with

$$
\varepsilon=\frac{1}{2}\left[\frac{1-\sqrt{ } k}{1+\sqrt{ } k}\right]
$$

All of the integer coefficients of the nome which will be required are found in Ferguson et al. ${ }^{3 \theta}$ When the number of terms used in this algorithm is discussed, it is assumed that one more term is taken in (D19) than in the sum in (D18). While not essential, this was the way the computation was done.

While this formula works well for a large parameter range, it behaves poorly for $k \approx f$ due to the fact that (D19) converges very slowly when $\varepsilon$ approaches $1 / 2$. The remedy 1 ies in the functional relation 


$$
q=\exp \left(-\pi \frac{K}{K}\right)
$$

where

$$
K^{*} \equiv K\left(k^{*}\right)
$$

There is a fundamental symmetry arising from (D21) which motivates a great deal of what follows. To obtain this symmetry relation, we note that

$$
\varepsilon^{\prime} \equiv \varepsilon\left(k^{\prime}\right)=\frac{1}{2}\left[\frac{1-V V\left[1-\left(k^{\prime}\right)^{2}\right]}{1+V V\left[1-\left(k^{\prime}\right)^{2}\right]}\right]=\frac{1}{2}\left[\frac{1-V k}{1+\sqrt{ } k}\right] .
$$

Further defining

$$
q^{\prime} \equiv q\left(\varepsilon\left(k^{\prime}\right)\right)=q\left(\varepsilon^{\prime}\right)
$$

gives the functional relation for $q^{\circ}$ as

$$
q^{\prime}=q\left(\sqrt{ }\left[1-k^{2}\right]\right)=\exp \left(-\pi \frac{K\left(\sqrt{ }\left[1-\left(1-k^{2}\right)\right]\right.}{K\left(\sqrt{ }\left[1-k^{2}\right]\right)}\right)=\exp \left(-\pi \frac{K(k)}{K(k)}\right) .
$$

Taking the $\log$ of (D21) and (D25) and multiplying the resulting equations leads to 


$$
\log (q) \log \left(q^{\cdot}\right)=\pi^{2}
$$

a relation already known to Davis. ${ }^{29}$ This important symmetry condition facilitates the computation of either $q$ or $q^{\prime}$ whenever they are difficult to compute directly. That is, when $k \approx 1$, the series (D19) converges very slowly, but the same series for $q^{\prime}$ will converge extremely rapidly with even one term because $k \approx 0$. Under these conditions, (D26) gives

$$
q=\exp \left(\frac{\pi^{2}}{\log \left(q^{\circ}\right)}\right)
$$

It is more sat isfactory yet to eliminate $q$ altogether from the computation by using (D21) and (D26):

$$
K(k)=-\frac{K\left(k^{\prime}\right) \log \left(q^{\prime}\right)}{\pi}=-\frac{\log \left(q^{\prime}\right)}{2}\left[1+2 \sum_{n=1}^{\infty}\left(q^{\prime}\right)^{n^{2}}\right]^{2}
$$

This is equivalent to a result given by Jahnke, Emde, anc Losch. ${ }^{53}$

Now the symmetry of relation (D26) implies that (D18) should be used on half of the parameter rane and (D28) on the other half. This is best understood by noting that $k=k^{\circ}$ when

$$
k^{2}=\frac{1}{2} \text {. }
$$


and that $K(k)=K^{\circ}=K\left(k^{\circ}\right)$ and $q=q^{\circ}=e^{-\pi}$ in this circumstance. Therefore, formulas (D18) and (D28) are identical under these conditions, and if the $\log$ is computed to comparable precision, the formulas will match term for term. In conjunction with the fact that formula (D18) converges better for small values of $k$ while (D28) converges better for small values of $k^{\prime}$, the identical behavior at $k^{2}=1 / 2$ implies that (D18) should be used for $k^{2} \leqslant 1 / 2$ and (D28) should be employed when $k^{2}>1 / 2$

\section{D.5 Comparison of the Algorithms for $\boldsymbol{K}$}

In order to compare the algorithms, it is important to compare the form in which they are actually implemented. For reasons of computational efficiency, all of the power series are computed in Horner series format. That 1s, the general power series can be written in the nested form

$$
\sum_{i=1}^{n} a_{\imath} x^{2}=a_{0}+x\left[a_{1}+x\left(a_{2}+\ldots .+a_{n} x\right) . . .\right] .
$$

This restructuring is applied direcily to the power series methods and the Hastings technique, as well as the series for the theta nome. For the series appearing in (D18), Horner's scheme is applied to the series separated into even and odd terms. This gives 


$$
\sum_{n=1}^{\infty} q^{n^{2}}=q\left[1+q^{8}(1+\ldots . .)\right]+q^{4}\left[1+q^{12}(1+\ldots . .)\right] .
$$

We now use the estimates given in Table D.1 for the number of cycles the required operations take on the CRAY-1. Note that the vector timings are in terms of cycles per operation for a vector of size 25, and that the performance would be considerably improved for larger vector sizes.

Table D.1 Timing estimates for the CRAY-1

$$
\text { Operation Scalar(cycles) Vector(cycles/result) }
$$

\begin{tabular}{lrl}
\hline & 7 & 1.3 \\
+ & 6 & 1.3 \\
$f$ & 32 & 2.3 \\
$\checkmark$ & 200 & 20. \\
$\log$ & 208 & 40.
\end{tabular}

For $k^{2} \leqslant 1 / 2$, the comparative execution speed of algorithms stable in this parameter range is given in Table D.2. Similarly, Table D.3 compares stable algorithms in the parameter range $k^{2}>1 / 2$. In these tables, the number of iterations or terms required to achieve a relative accuracy of $10^{-13}$ was deduced 
from numerical experiments and graphs similar to Figures D.1 through D.4. Because the Landen transformation and infinite product methods cost essentially the same number of cycles as the arithmetic-geometric mean. they are not included.

Table D.2 Cost of computing $K(k)$ to 13 significant digits for $0<k^{2} \leqslant 1 / 2$.

Method Iterations/terms Scalar(cycles) Vector(cycles/result)

Arlth.-Geo. Mean

4

900

98

Power series (D16)

41

550

105

Hastings form

8

470

86

The ta Nome (D18)

3

390

53

The timing figures given in these tables are approximate; they can vary depending upon the operating environment. Consequently, the timings for the Hastings form method and the functional theta nome relation should be regarded as roughly the same, but they would both be clearly faster than either of the other methods. Another factor to be kept in mind is that the estimates given in Table D.1 will vary from computer to computer, and that a method which shows up as slightly superior in the timings found here might end up slightly inferior on 
another machine. Therefore, the conclusions drawn here are valid only for the CRAY-1 and machines with very similar floating point behavior; adjustments in the data would be necessary on other computers.

Table D.3 Cost of computing $K(k)$ to 13 significant digits for $1 / \sqrt{ } 2<k<1-10^{-13}$.

Method Iterations/terms Scalar(cycles) Vector(cycles/result)

$\begin{array}{lccc}\text { Arith.-Geo. Mean } & 7 & 1600 & 170 \\ \text { Power series (D17) } & 41 & 1300 & 249 \\ \text { Hastings form } & 8 & 470 & 86 \\ \text { Functional (D28) } & 3 & 600 & 93\end{array}$

Combining the information from Tables D.2 and D. 3, it appears that a hybrid method based on the theta function nome, i.e., (D18) and (D28), would perform the best. However, when computing in vector mode, the number of cycles required to $\mathrm{f}$ ind $K$ for any given $k$ is the sum of each technique involved in the hybrid. This unfortunate situation arises because the CRAY-1 architecture does not allow operand masking; that is, only results can be masked (merged) to select the single desired result, but each result must be available, and so each 
of the methods making up the hybrid algorithm must have been computed. This leaves the hybrid theta nome algorithm as costing 146 cycles per result. slightly more expensive than the Hastings form method.

We now turn to some graphs to show how some of these algorithms perform for given values of $k$. Before doing so, it should be noted that the Landen transformation and inf inite product methods gave results so numerically close to those of the arithmetic-geometric mean that they were graphically indistinguishable. Therefore, they do not appear in the graphs.

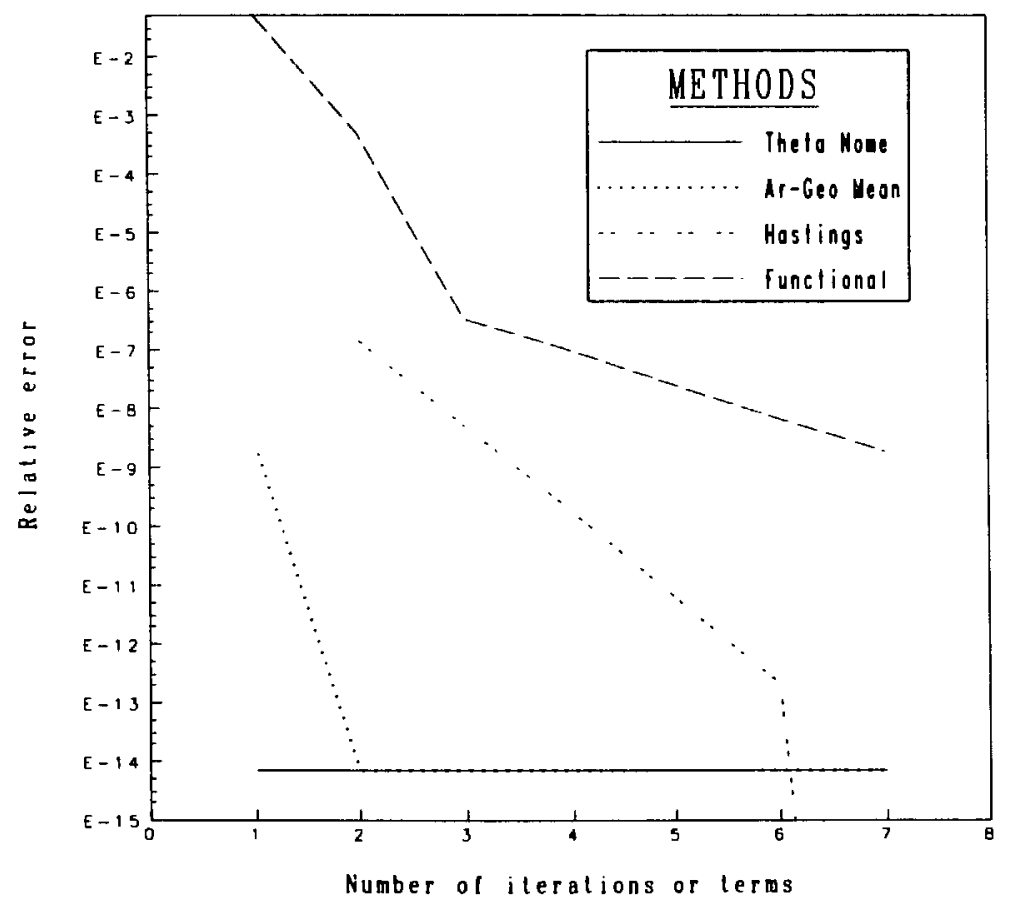

Figure D.1. Convergence characteristics of several methods for finding $K$ at $k=0.02$. 
In Figure D.1, the speed of convergence of the theta nome is dramatic: only one term is required to obtain machine precision for $k=0.02$. Also, the arithmetic-geometric mean is converging much faster than the Hastings method. Lastly, the functional theta nome relation, which is not designed to do well for small $k$, is indeed doing poorly.

Figure D.2 shows that near the center of the parameter range $(k=0.7)$ the theta nome and its functional counterpart do perform nearly identically. While the performance of the arithmetic-geometric mean is noi quite as good as in

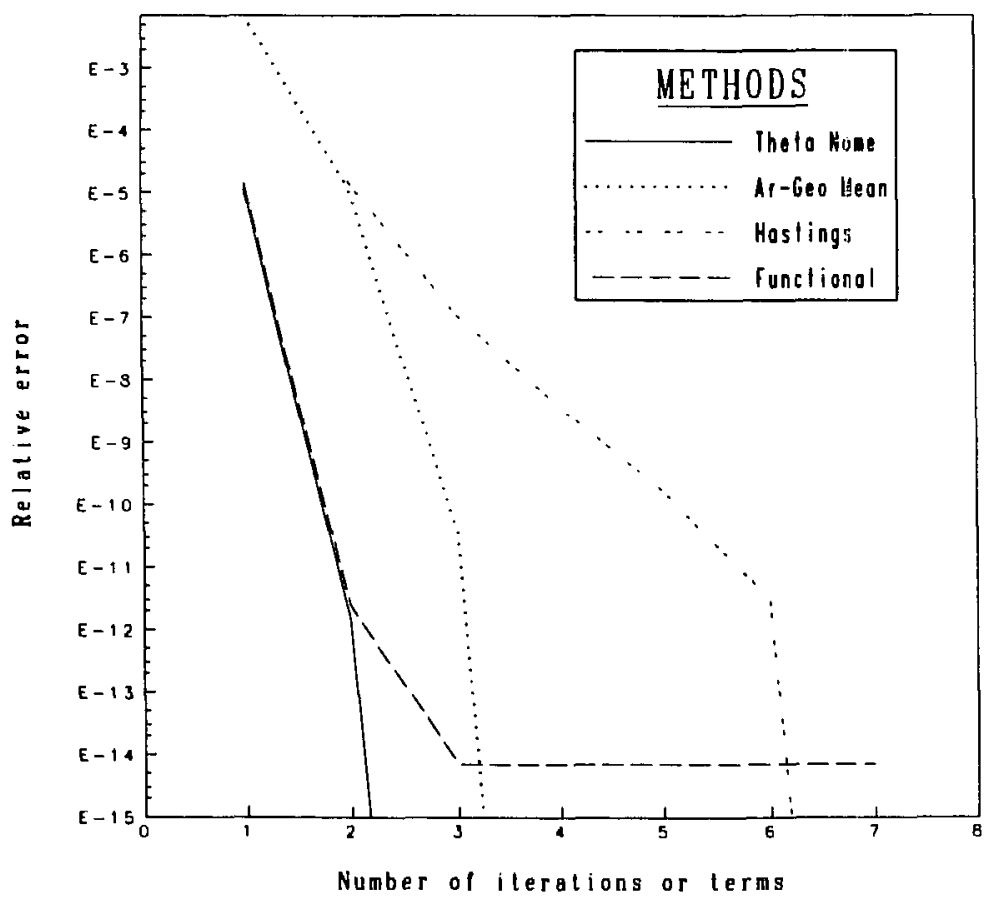

Figure D.2. Convergence characteristics of several methods for finding $K$ at $k=0.7$.

Figure D.1, the convergence appears to acceierate from step to step. On the 
other hand, the Hastings form method still takes a full 7 terms to converge to 13 significant figures. This behavior persists in Figure D.3, where the arithmetic-geometric mean's convergence is weakening. The functional theta nome relation is now dominant, requiring only two terms for convergence. Also, it is

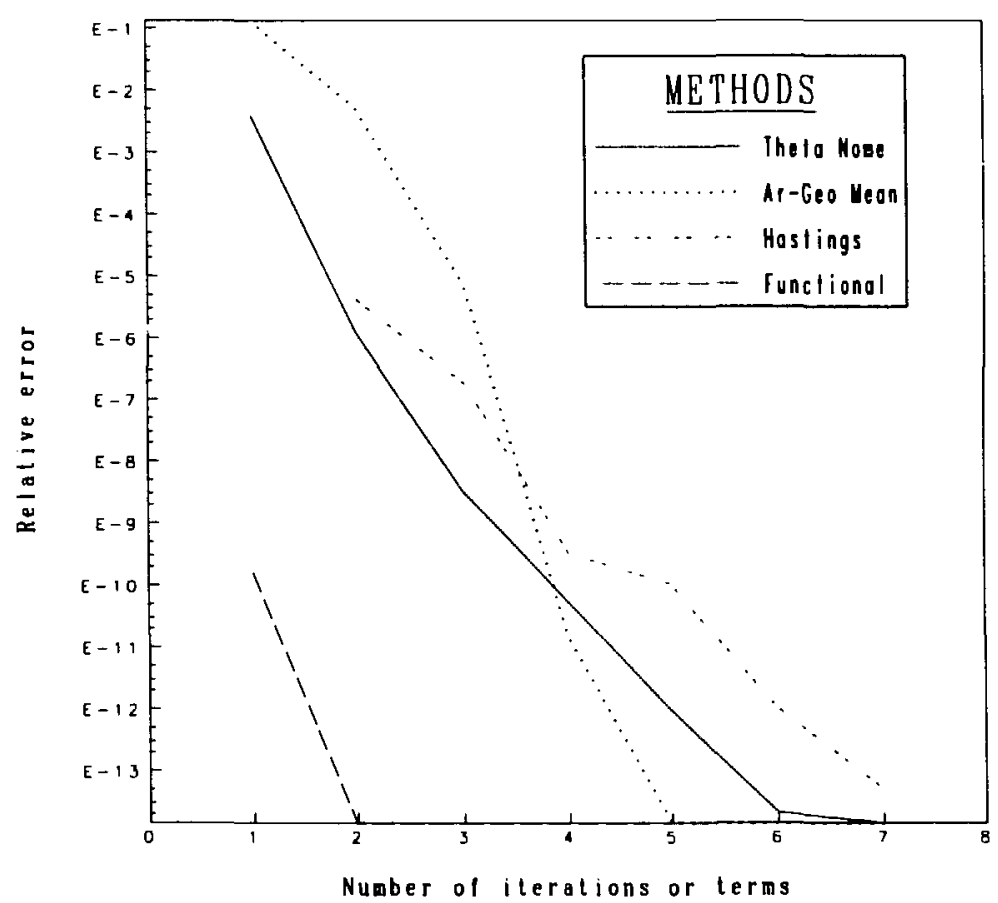

Figure D.3. Convergence characteristics of several methods for finding $K$ at $k=0.98$.

clear that the theta nome should not be usd for $k$ much greater than 0.98 because its convergence is rapidly becoming poor. When $k=0.9999999$, for example, only very slow convergence is left in the theta nome series. This slow convergence is evident in Figure D.4. And just as the theta nome was dominant for $k \times 41$, so now the functional theta nome relation is dominant for $k \approx 1$. Also, the Hastings form is beginning to come into its own in this regime, although it still takes 6 
terms to achieve 13 significant digits. The power series relation (D17) would also do well in this parameter range, but not quite as well as the Hastings method.

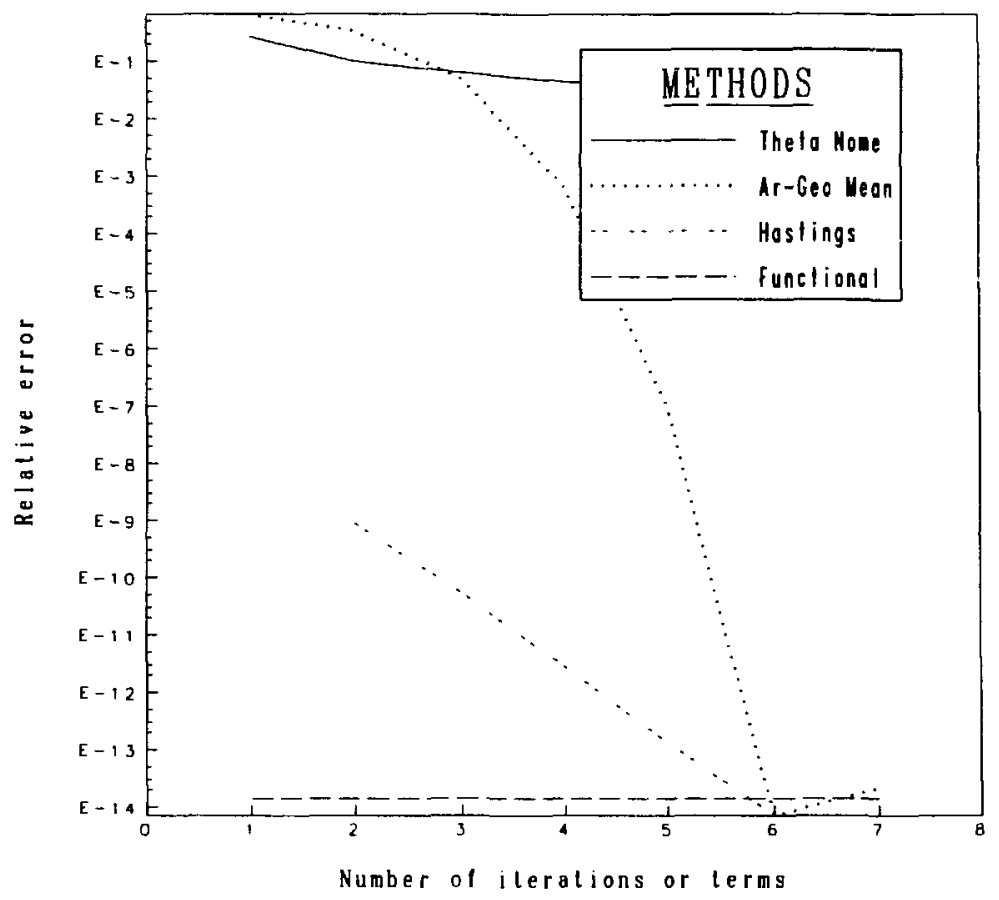

Figure D.4. Convergence characteristics of several methods for finding $K$ at $k=0.9999999$.

\section{D.6 Algorithms for $E$}

For $E$, just as for $K$, the most popular algorithm is the arithmetic-geometric mean. In addition to the iterates defined by (D6), there is the sequence 


$$
c_{\imath}=\frac{1}{2}\left(a_{\imath-1}-b_{\imath-1}\right)
$$

where $c_{0}=k$. The iteration is terminated as before, leading to the approximate value for $E$ of

$$
E(k) \approx \frac{\pi}{2 a_{N}}\left(1-\frac{1}{2} \sum_{i=0}^{N} 2^{i} c_{i}^{2}\right)
$$

There is also a Landen transformation very similar to that for $K$. The iterations described by (D8) still apply: only the initialization $b_{0}=k^{-\varepsilon}$ is different.

A new infinite product iterative scheme for $E$ was recently found by Ferguson. ${ }^{36}$ It is

$$
E(k)=\prod_{n=0}^{\infty} \frac{1+R^{n}(k)}{2}+\sum_{n=0}^{\infty} L\left(R^{n}(k)\right) \prod_{m=0}^{n-1} \frac{1+R^{m}(k)}{2}
$$

where empty products equal 1. This iteration would be terminated when $R^{n}(k)=1$ to the required precision. While this method does very well for $k \approx 1$. its complexity makes it difficult to unfold the iterations to allow the algorithm to vectorize. 
Although there is no singularity in $E$ at $k=1$, it nonetheless presents difficulties there, just as $K$ does. And in the same spirit of finding expressions which have the correct asymptotic behavior for $K$ near $k=1$, there are Legendre forms for $E$. A "modified" Legendre form is ${ }^{21}$

$$
E(k)=E_{1}\left(k^{\prime}\right)+E_{2}\left(k^{\circ}\right) \log \left(\frac{1}{\left(k^{\prime}\right)^{2}}\right) .
$$

with

$$
\begin{aligned}
E_{1}\left(k^{\prime}\right)= & +\frac{\left(k^{\prime}\right)^{2}}{2}[\log 4-1] \\
& +\sum_{n=2}^{\infty}\left[\frac{(2 n-1) ! !}{2^{n} n !}\right]^{2} \frac{2 n}{2 n-1}\left[\log 4-2 \sum_{j=1}^{2 n-2} \frac{(-1)^{j}}{j}-\frac{1}{2 n-1}+\frac{1}{2 n}\right]\left(k^{\prime}\right)^{2 n} \\
E_{2}\left(k^{\prime}\right)= & \frac{\left(k^{\prime}\right)^{2}}{4}+\frac{1}{2} \sum_{n=2}^{\infty}\left[\frac{(2 n-1) ! !}{2^{n} n !}\right]^{2} \frac{2 n}{2 n-1}\left(k^{\prime}\right)^{2 n} .
\end{aligned}
$$

As with $K$, some Chebyshev polynomial approximations for $E$, and $E_{z}$ given by Cody $^{22}$ were tried. They were still quite slowly convergent and became unstable for $k \approx 1$ due to the alternating signs of successive terms causing nearly complete cancellation. The Hastings form given again by Cody 21 suffered no such difficulty. This form is given by

$$
E(k) \approx \sum_{i=0}^{n} c_{i}\left(k^{\prime}\right)^{2 i}+\log \left(\frac{1}{\left(k^{\prime}\right)^{2}}\right) \sum_{i=0}^{m} d_{i}\left(k^{\prime}\right)^{2 i} \text {, }
$$


where

$$
c_{0}=1, \quad \sum_{i=0}^{n} c_{i}=\frac{\pi}{2}, d_{0}=0 .
$$

$n=m$ also yields the best numerical properties in this case, and because of the strict positivity of all of the $c_{i}{ }^{\prime} s$ and $d_{i}^{\prime} s^{\prime}$, there is no difficulty with alternating signs.

As there are power series for $E$ which perform well in limited parameter regimes, two are given here. For $k<<1$, the appropriate power series is

$$
E(k)=\frac{\pi}{2}\left\{1-\sum_{n=1}^{\infty}\left[\frac{(2 n-1) ! !}{2^{n} n !}\right]^{2} \frac{k^{2 n}}{2 n-1}\right\}
$$

In the difficult area of $k \approx 1$, there is the series

$$
E\left(k^{\prime}\right)=1+\sum_{n=1}^{\infty}\left[\frac{(2 n-1) ! !}{2^{n} n !}\right]^{2} \frac{2 n}{2 n-1}\left[\log \frac{4}{k^{\cdot}}-2 \sum_{l=1}^{n} \frac{1}{(2 l-1) 2 l}+\frac{1}{(2 n-1) 2 n}\right]\left(k^{\prime}\right)^{2 n}
$$

Their slow convergence behavior elsewhere is evident in tables D.4 and D.5.

Paralleling again the mothods for $K$. there are theta nome expressions for $E$. Byrd and Friedman give ${ }^{15}$ 


$$
\begin{aligned}
E & =\frac{\pi}{2}\left[\vartheta_{3}(0)\right]^{2}\left[1-\frac{\Theta^{\prime}(0)}{\Theta(0)}\right] \\
& =K-\frac{2 \pi^{2}}{K} \frac{\sum_{n=1}^{\infty}(-1)^{n+1} n^{2} q^{n^{2}}}{1+2 \sum_{n=1}^{\infty}(-1)^{n} q^{n^{2}}} .
\end{aligned}
$$

A similar expression in terms of strictly positive quantities is ${ }^{53}$

$$
\begin{aligned}
E & =-\frac{1}{2 \pi \vartheta_{3}^{2}(0)} \frac{\vartheta_{2}^{\prime}(0)}{\vartheta_{2}(0)} \\
& =\frac{\pi}{2} \frac{\sum_{n=1}^{\infty}(2 n-1)^{2} q^{n(n-1)}}{\left[\sum_{n=1}^{\infty} q^{n(n-1)}\right]\left[1+2 \sum_{n=1}^{\infty} q^{n^{2}}\right]^{2}} .
\end{aligned}
$$

Unfortunately, because (D19) converges so slowly for $k \approx t$, these formulas cannot be used in that parameter range. So we again seek a functional relationship. Using Legendre's relation,

$$
K E^{\prime}+K^{\prime} E-K K^{\prime}=\frac{\pi}{2}
$$

we $f$ ind 


$$
E=\frac{\frac{\pi}{2}+K\left(K^{\prime}-E^{\prime}\right)}{K^{\prime}}
$$

In this equation, the primes indicate that the argument of the function is $k^{\prime}$. lising (D41), we obtain

$$
K^{\prime}-E^{\prime}=\frac{2 \pi^{2}}{K^{\prime}}\left(\frac{\sum_{n=1}^{\infty}(-1)^{n+1} n^{2}\left(q^{\prime}\right)^{n^{2}}}{1+2 \sum_{n=1}^{\infty}(-1)^{n}\left(q^{\prime}\right)^{n^{2}}}\right) .
$$

Any further dependence on $q$ in (D44) is removed by using the relation

$$
\frac{K}{K^{\cdot}}=-\frac{\log \left(q^{\prime}\right)}{\pi}
$$

These two equations. (D45) and (D46), can be used in (D44) to obtain

$$
E=\frac{1}{K^{\prime}}\left\{\frac{\pi}{2}-2 \pi \log \left(q^{\prime}\right)\left(\frac{\sum_{n=1}^{\infty}(-1)^{n+1} n^{2}\left(q^{\prime}\right)^{n^{2}}}{1+2 \sum_{n=1}^{\infty}(-1)^{n}\left(q^{\prime}\right)^{n^{2}}}\right)\right\}
$$

It can be shown that this is equivalent to a result given by Jahnke, Emde, and Losch. ${ }^{53}$ In addition, it should be noted that $K^{\prime}$ is evaluated using (D18) with $q$ replacing $q$. 
Because (D47) will converge fast when $k \approx 1\left(k^{\prime}<<1\right)$, it should be used in that regime. To determine where to divide the parameter range between (D41) and (D47), consider the situation when $k=k^{\prime}$. In that case, $K=K^{\prime}$ as noted before. The value is 1.854075 , so the iterations in the two formulas are not exactly the same. Nevertheless, it turns out that they are numerically nearly identical for a comparable number of terms, and therefore, $k^{2}=1 / 2$ is once again used as the value separating the two regions.

\section{D.7 Comparison of the Algorithms for $\boldsymbol{E}$}

As was the case in the series methods for finding $K$. Horner's scheme is used to evaluate the series arising in algorithms for $F$. There is only one new series decomposition which might need some elucidation. It is

$$
\sum_{n=1}^{\infty}(-1)^{n+1} n^{2} q^{n^{2}}=q\left[1+q^{8}(9+\ldots . .)\right]+q^{4}\left[4+q^{12}(16+\ldots . .)\right]
$$

Thus, all series of squared powers of $q$ require the same number of operations.

Because the parameter range for $k$ has again. been divided into two parts, we consider separately the performance of algorithms in each region. Once again, the Landen transformation results were graphically indistinguishable from those of the arithmetic-geometric mean. Hence, they are omitted from the analysis here. 
Table D.4 Cost of computing $E(k)$ to 13 significant digits for $0<k^{2} \leqslant 1 / 2$.

Me thod

Iterations/terms
Scalar(cycles)

\section{Vector(cycles/result)}

$\begin{array}{lccc}\text { Arith.-Geo. Mean } & 4 & 1100 & 128 \\ \text { Product } & 5 & 13000 & 1400 \\ \text { Power series (D39) } & 41 & 550 & 105 \\ \text { Hast ings form } & 8 & 470 & 86 \\ \text { The ta nome (D41) } & 3 & 650 & 99\end{array}$

In Table D.4, the execution speed results for $k^{2}<1 / 2$ are presented. Table D.5 contains the results for $1 / \sqrt{ } 2<k<1-10^{-13}$. In each case, the number of terms or iterations required to reduce the relative error to less than $10^{-13}$ is indicated. From these tables, it is clear that the Hastings form method is most rapidly computed in both parameter regions, although the theta nome and its functional counterpart are not too far behind. Observe that a hybrid method based on the theta nome would require 241 cycles per result in vector mode, the same order of magnitude as the arithmetic-geometric mean vector speed, both being clearly slower than the 86 cycles per result speed of the Hastings form. However, these timings all apply to the computation of $E$ only. 
Table D.5 Cost of computing $E(k)$ to 13 significant digits for $1 / \sqrt{ } 2<i<1-10^{-13}$.

Me thod

Iterations/terms Scalar(cycles) Vector(cycles/result)

Arith.-Geo. Mean

8

2100

249

Produc $t$

5

13000

1400

Power series (D40)

41

1300

249

Hastings form

8

470

86

Functional (D47)

3

900

142

A different picture emerges if both $K$ and $E$ are required for a given value of $k$ (or set of $k^{\prime} s$ ). This arises because the arithmetic-geometric mean and the theta nome methods must essentially find $\boldsymbol{K}$ in order to find $E$. While there is some duplication in the computation of $K$ and $E$ by the series methods, there is not enough duplication to lead to the same order of improvement. Table D.6 presents the three fastest methods under these conditions. Notably, the arithmetic-geometric mean is now of comparable speed to the theta nome hybrid methnd. However, both are slightly slower than the Hastings form method. 
Table D.6 Cost of computing $K(k)$ and $E(k)$ to 13 significant digits for $0<k<1-10^{-13}$.

Method Iterations/terms Scalar(cycles) Vector(cycles/result)

Arith.-Geo. Mean

8

2100

238

Hastings form

8

940

172

Theta nome hybrid

3

$650\left(k^{2}<1 / 2\right)$

241

$900\left(k^{2}>1 / 2\right)$

The picture for the scalar mode of calculation is now considerably different. The theta nome hybrid is the fastest with the Hastings form method being only slightly slower. Interestingly, the method most widely used, the arithmetic-geometric mean, is over two times slower! This assumes a fixed number of iterations for the arithmetic-geometric mean, and as will be found from examining the figures, it of ten performs better. Consequently, the speed will depend upon the particular value of $k$ involved; for small $k$, the number of cycles used is about 550, rising to 2100 for $k \approx 1$. But the improvement for small $k$ alsu occurs in the theta nome, although some extra subdivisioning of the parameter range would be required to take full advantage of the improved convergence behavior there as well as that which occurs in the functional theta 
nome method for $k \approx 1$. Since only one term in the theta nome is required for small $k$, the scalar speed of computing $E$ and $K$ in that region would be 450 cycles. Therefore, the scalar performance of the hybrid theta nome method can be made uniformly better that the arithmetic-geometric mean through three subdivisions of each region (to take full advantage of the fewest number of terms required at any value of $k$ ). By contrast, as the Hastings form method converges in a uniform manner over almost the entire parameter range, there is no way to improve its scalar performance.

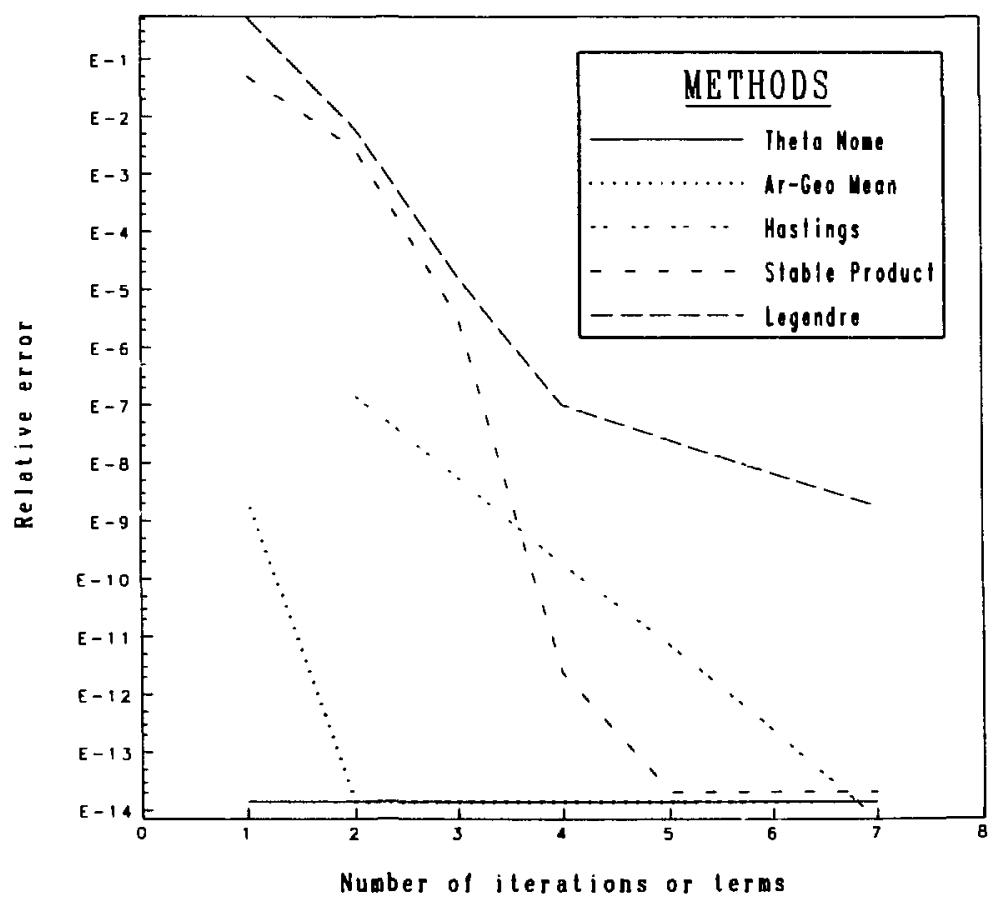

Figure D.5. Convergence characteristics of several methods for finding $E$ at $k=0.02$. 
Turning next to some graphical results, we consider the speed of convergence of some of the methods in terms of the number of terms or iterations employed. First, for $k=0.02$, Figure D.5 shows how in only one iteration the theta nome method has converged to better than machine precision. The arithmetic-geometric mean is not quite as fast, requiring two iter, 'ions to converge. While very slow computationally. the speed of convergence of the infinite product method improves with more iterations, so that for machine accuracy, it requires fewer terms than the Hastings method. Because, as previously noted, the $q$ series converges very slowly for small $k$, the functional method (here labelled the "Legendre" method) performs poor'y, and Figure D.5 confirms that fact.

Figure D.6 moves us near the center of the parameter range $(k=0.7)$. An: as expected, the theta nome method and its functional counterpart behave nearly the same. The arithmetic-geometric mean is not doing quite as well, while the inf irite product method is converging to machine accuracy one iteration sooner. Finally, the Hastings form method is performing much as in the earlier graph. In Figure D.7, at $k=0.98$, the Hastings form method is again roughly the same as in Figure D.5. On the other hand, the functional theta nome method is becoming dominant, while the theta nome method itself weakens. Also weakening is the arithmetic-geometric mean, which is to be expected as $k$ gets close to 1 . The inf inite product method is continuing to show stable behavior by converging in four iterations. 


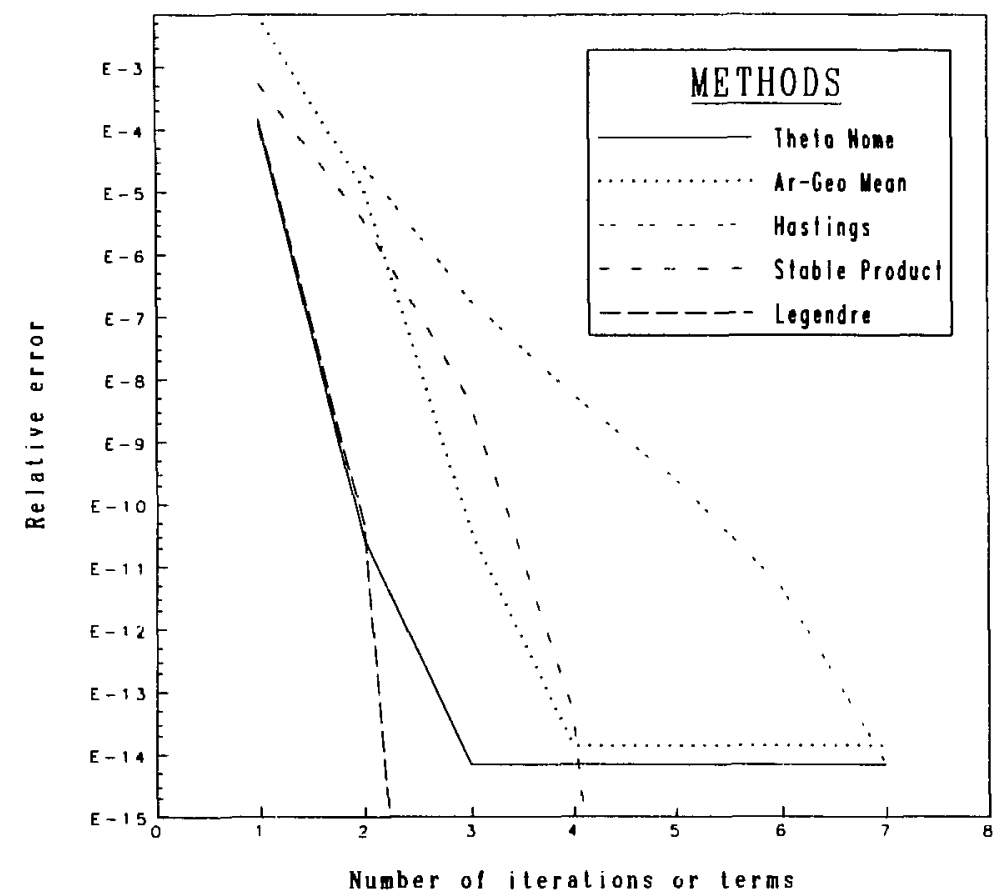

Figure D.6. Convergence characteristics of several methods for finding $E$ at $k=0.7$.

As we approach very close to the singularity in $K$, the picture changes substantially. For example, at $k=0.99999999$, Figure D.8 shows that the functional method has more than converged. Also very interesting is the much improved convergence of the infinite product iterations and the Hastings form method. Both of these methods continue to perform better and better as $k$ gets closer to 1. And at the same time, the theta nome method converges more and more slowly, again showing that it is not a viable technique for $k \approx 1$. Finally. observe that the arithmetic-geometric mean does not completely converge to machine accuracy in seven iterations. This lack of convergence is caused by the fact that as $K$ is becoming very large, it is multiplying a very small number, 


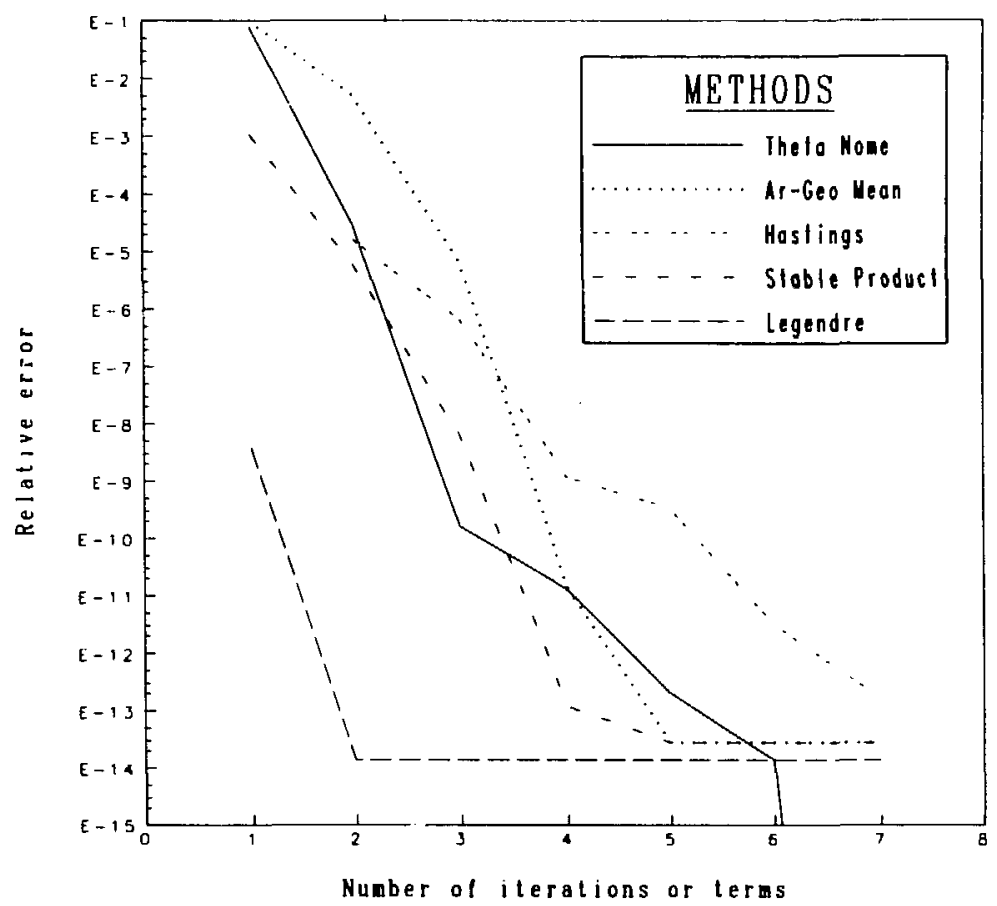

Figure D.7. Convergence characteristics of several methods for finding $E$ at $k=0.98$.

and this small number is computed as a difference of two numbers very close to 1. Thus, a number of digits can be lost in this method if the singularity is approached close enough.

\section{D.8 Concluding Remarks}

From the results presented in sections 4 and 6 , no one technique stands out clearly above all the others for all criteria. However, it is of great interest to note that while the arithmetic-geometric mean has been widely accepted as the method of choice. it comes in no better than third place in the scalar and vector speed competition for finding $K$ and $E$ separately. Even when 


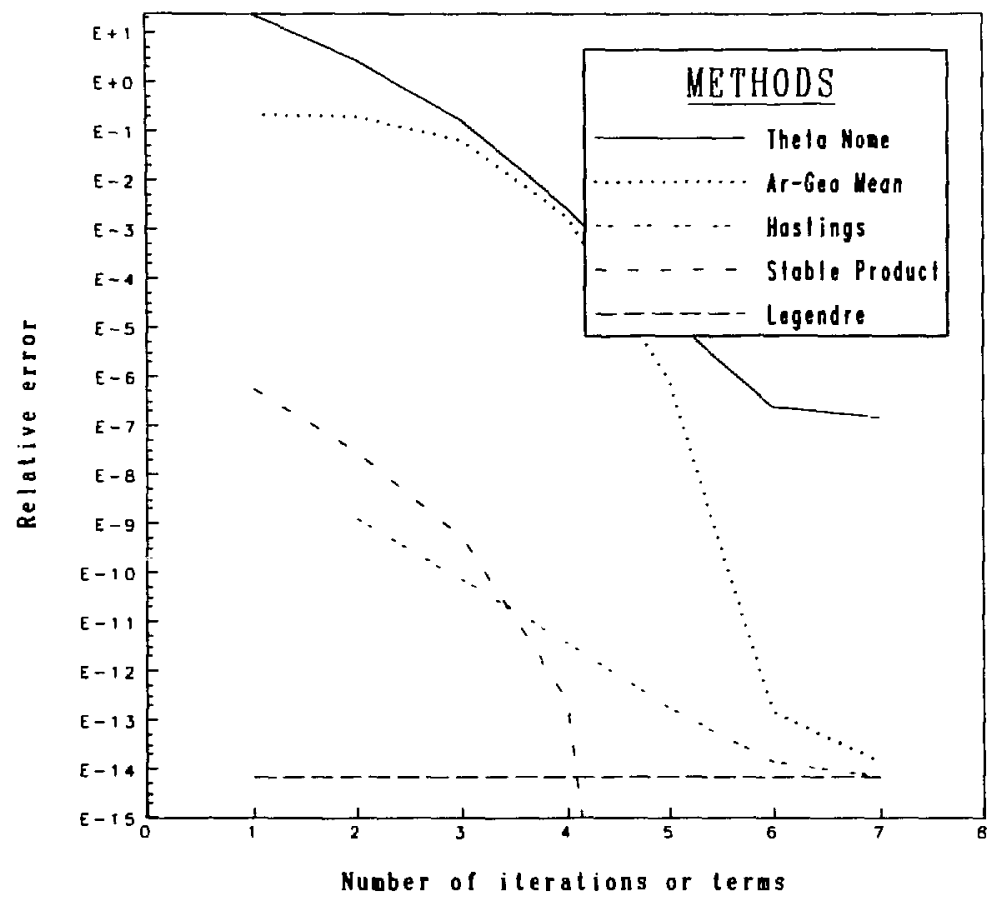

Figure D.8. Convergence characteristics of several methods for finding $E$ at $k=0.99999999$.

it has an advantage of computing $K$ and $E$ simultaneously, it is in second place in scalar computation for small values of $k$ and in third place elsewhere. In vector computation for finding $K$ and $E$ simultaneously, it is tied for second place. However, because it is somewhat artificial to unfold an intrinsically iterative technique to a given level so as to allow the iterations to vectorize. the vectorized form of the arithmetic-geometric mean should not be accepted. We reject the vectorized form because implementing a vector form of the algorithm on a different parallel computer with a different precision requires the unfolding be done to a different level. This leads to unportable software, which should not be tolerated when the software is this basic. Another drawback 
of the arithmetic-geometric mean was pointed out in section 6 . And that drawback was the numerical instability this method suffers from in computing $E$ very near $k=1$. Consequently, we recommend abandoning the arithmetic-geometric mean for the computation of $K$ and $E$.

In the speed analysis, the fact that current computer architectures permitting parallel computations do not allow operand masking leaves the Hastings form method as clearly fastest for vector computations. If operand masking did exist, then the theta nome hybrid methods would be fastest for finding $K$ in half of the parameter range (and roughly tied in the other half), and fastest for finding both $K$ and $E$ over the entire range of $k$ values.

There is a restricted set of cases where this speedup can be obtained without the operand masking capability. These are the cases where it is efficieni and convenient to separate the argument vector into two vectors, one for $k^{2} \leqslant 1 / 2$ and the other for $k^{2}>1 / 2$. Perhaps it is known that only one case will occur. In either event, these special situations allow the theta nome hybrid methods to be most efficient in the vector mode of operation on the CRAY-1

In the scalar mode of computation, there is no clearcut winner. For $k^{2}<1 / 2$, the theta nome method $\mathrm{f}$ inds $K$ fastest. For the other half of $k$ values, the Hastings form method is superior. It also does the best job in computing $E$ alone. But when finding $K$ and $E$ simultaneously, the theta nome hybrid techniques are superior. 
Fortunately. the convergence analysis is not muddled by this mixture of results. Over the entire range of $k$ values for both $K$ and $E$, the theta nome hybrid methods converge fastest. So where does this leave us? In a general setting, it is probably a tossup to decide whether to use the theta nome techniques or the Hastings form series. In specific applications, the choice should be based upon the speed and implementation results given here.

\section{D.9 Acknowledgment}

Thanks to Dale E. Nielsen, Jr. for pointing out the hybrid theta nome methods already given by Jahnke, Emde, and Losch. He had previously implemented these methods and found the same convergence characteristics described here. 


\section{APPENDIX E}

\section{FORTRAN OUTPUT}

This appendix contains the 1 isting of the four files of FORTRAN output generated by the second example in Chapter 6. We begin with "gcook;middll mid":

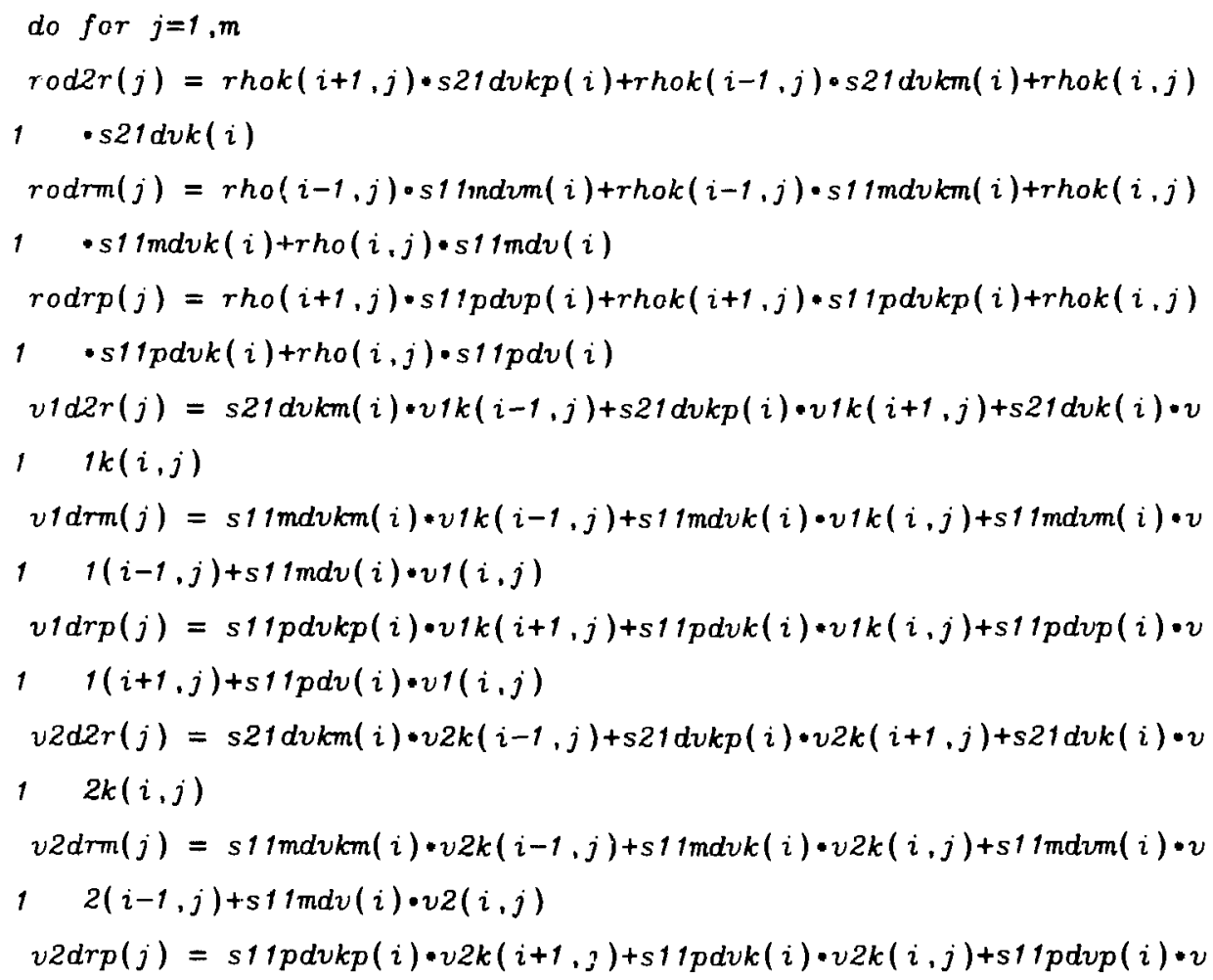


$v 3 d 2 r(j)=s 21 d v k m(i) \cdot v 3 k(i-1, j)+s 21 d v k p(i) \cdot v 3 k(i+1, j)+s 21 d v k(i) \cdot v$

$13 k(i, j)$

$v 3 d r m(j)=s 11 m d v k m(i) \cdot v 3 k(i-1, j)+s 11 m d v k(i) \cdot v 3 k(i, j)+s 11 m d v m(i) \cdot v$

$1 \quad 3(i-1, j)+s 11 m d v(i) \cdot v 3(i, j)$

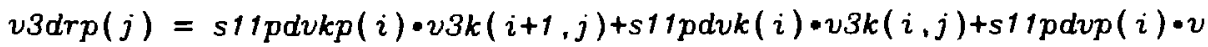

$13(i+1, j)+s 11 p d v(i) \cdot v 3(i, j)$

$t d 2 r(j)=\operatorname{s21dvkm}(i) \cdot t k(i-1, j)+s 21 d v k p(i) \cdot t k(i+1, j)+s 21 d v k(i) \cdot t k(i$

$1 . j)$

$t \operatorname{drm}(j)=\operatorname{s11mavkm}(i) \cdot t k(i-1, j)+s 11 m \operatorname{dvk}(i) \cdot t k(i, j)+s 11 m d v m(i) \cdot t(i-$

$1 \quad 1, j\}+s 11 m d v(i) \cdot t(i, j)$

$t d r p(j)=s 11 p d v k p(i) \cdot t k(i+1, j)+s 11 p d v k(i) \cdot t k(i, j)+s 11 p d u p(i) \cdot t(i+$

$1 \quad 1, j)+s 11 p d v(i) \cdot t(i, j)$

$b 1 d 2 r(j)=b 1 k(i+1, j) \cdot s 21 d v k p(i)+b 1 k(i-1, j) \cdot s 21 d v k m(i)+b 1 k(i, j) \cdot s 2$

$1 \quad 1 \operatorname{dvk}(i)$

$b 1 d r m(j)=b 1(i-1, j) \cdot s 11 m d v m(i)+b 1 k(i-1, j) \cdot s 11 m d v k m(i)+b 1 k(i, j) \cdot s 1$

$1 \quad 1 m d v k(i)+b 1(i, j) \cdot s 11 m d v(i)$

$b 1 \operatorname{drp}(j)=b 1(i+1, j) \cdot s 11 p d v p(i)+b 1 k(i+1, j) \cdot s 11 p d v k p(i)+b 1 k(i, j) \cdot s 1$

$1 \quad 1 p d v k(i)+b 1(i, j) \cdot s 11 p d v(i)$

$b 2 d 2 r(j)=b 2 k(i+1, j) \cdot s 21 d u k p(i)+b 2 k(i-1, j) \cdot s 21 d v k m(i)+b 2 k(i, j) * s 2$

$1 \quad 1 d v k(i)$

$b 2 d r m(j)=b 2(i-1, j) \cdot s 11 m d v m(i)+b 2 k(i-1, j) \cdot s 11 m d v k m(i)+b 2 k(i, j) \cdot s 1$

$11 m d v k(i)+b 2(i, j) \cdot s 11 m d v(i)$

$b 2 d r p(j)=b 2(i+1, j) \cdot s 11 p d v p(i)+b 2 k(i+1, j) \cdot s 11 p d v k p(i)+b 2 k(i, j) \cdot s 1$

$11 p d v k(i)+b 2(i, j) \cdot s 11 p d v(i)$

$b 3 a 2 r(j)=b 3 k(i+1, j) \cdot s 21 d v k p(i)+b 3 k(i-1, j) \cdot s 21 d v k m(i)+b 3 k(i, j) \cdot s 2$

$1 \quad 1 d v k(i)$

$b 3 d r m(j)=b 3(i-1, j) * s 11 m d v m(i)+b 3 k(i-1, j) \cdot s 11 m d v k m(i)+b 3 k(i, j) \cdot s 1$

$1 \quad 1 \operatorname{mdvk}(i)+b 3(i, j) \cdot s 11 m d v(i)$

$b 3 d r p(j)=b 3(i+1, j) \cdot s 11 p d v p(i)+b 3 k(i+1, j) \cdot s 11 p d v k p(i)+b 3 k(i, j) \cdot s 1$

$11 p d v k(i)+b 3(i, j) \cdot s 11 p d v(i)$

$\operatorname{rhs}(j, 9, i)=-\operatorname{cupkmk}(i) \cdot \operatorname{rhok}(i-1, j)-\operatorname{cupkpk}(i) \cdot \operatorname{rhok}(i+1, j)-\operatorname{rhok}(i, j$

$1)-\operatorname{cupnomk}(i) \cdot \operatorname{rho}(i-1, j)-\operatorname{cupupk}(i) \cdot \operatorname{rho}(i+1, j)-\operatorname{cupuk}(i) \cdot \operatorname{rho}(i, j)$

$\operatorname{rhs}(j, 10, i)=-\operatorname{cupkmk}(i) \cdot v 1 k(i-1, j)-\operatorname{cupkpk}(i) \cdot v 1 k(i+1, j)-v 1 k(i, j)-$ 

$\operatorname{rhs}(j, 11, i)=-\operatorname{cupkmk}(i) \cdot v 2 k(i-1, j)-\operatorname{cupkpk}(i) \cdot v 2 k(i+1, j)-v 2 k(i, j)-$ $1 \operatorname{cuprank}(i) \cdot v 2(i-1, j)-\operatorname{cupnepk}(i) \cdot v 2(i+1, j)-\operatorname{cupuk}(i) \cdot v 2(i, j)$ $\operatorname{rhs}(j, 12, i)=-\operatorname{cupkmk}(i) \cdot v 3 k(i-1, j)-\operatorname{cupkpk}(i) \cdot v 3 k(i+1, j)-v 3 k(i, j)-$ $1 \quad \operatorname{cupnank}(i) \cdot v 3(i-1, j)-\operatorname{cupupk}(i) \cdot v 3(i+1, j)-\operatorname{cupuk}(i) \cdot v 3(i, j)$ $\operatorname{rhs}(j, 13, i)=-\operatorname{cupkmk}(i) \cdot t k(i-1, j)-\operatorname{cupkpk}(i) \cdot t k(i+1, j)-t k(i, j)-\operatorname{cup}$ $1 \quad \operatorname{umk}(i) \cdot t(i-1, j)-\operatorname{cupupk}(i) \cdot t(i+1, j)-\operatorname{cupuk}(i) \cdot t(i, j)$ $\operatorname{rhs}(j, 14, i)=-b 1(i+1, j) \cdot \operatorname{cupupk}(i)-b 1(i-1, j) \cdot \operatorname{cupronk}(i)-b 1(i, j) \cdot$ cup $1 u k(i)-b 1 k(i+1, j) \cdot \operatorname{cupkpk}(i)-b 1 k(i-1, j) \cdot \operatorname{cupkmk}(i)-b 1 k(i, j)$ $\operatorname{rhs}(j, 15, i)=-b 2(i+1, j) \cdot \operatorname{cupupk}(i)-b 2(i-1, j) \cdot \operatorname{cupromk}(i)-b 2(i, j) \cdot \operatorname{cup}$ $1 u k(i)-b 2 k(i+1, j) \cdot \operatorname{cupkpk}(i)-b 2 k(i-1, j) \cdot \operatorname{cupkmk}(i)-b 2 k(i, j)$ $\operatorname{rhs}(j, 16, i)=-b 3(i+1, j) \cdot \operatorname{cupupk}(i)-b 3(i-1, j) \cdot \operatorname{cupumk}(i)-b 3(i, j) \cdot \operatorname{cup}$ $1 u k(i)-b 3 k(i+1, j) \cdot \operatorname{cupkpk}(i)-b 3 k(i-1, j) \cdot \operatorname{cupkmk}(i)-b 3 k(i, j)$ $c(j, 9,1)=\operatorname{cupmank}(i)$ $c(j, 10,2)=\operatorname{cupnamk}(i)$ $c(j, 11,3)=\operatorname{cupronk}(i)$ $c(j, 12,4)=\operatorname{cupronk}(i)$ $c(j, 13.5)=\operatorname{cupronk}(i)$ $c(j, 14,6)=\operatorname{cupromk}(i)$ $c(j, 15,7)=\operatorname{cupromk}(i)$ $c(j, 16,8)=\operatorname{cupromk}(i)$ $c(j, 9,9)=\operatorname{cupkmk}(i)$ $c(j, 10,10)=\operatorname{cupkmk}(i)$ $c(j, 11,11)=\operatorname{cupkmk}(i)$ $c(j, 12,12)=\operatorname{cupkmk}(i)$ $c(j, 13,13)=\operatorname{cupkmk}(i)$ $c(j, 14,14)=\operatorname{cupkmk}(i)$ $c(j, 15,15)=\operatorname{cupkmk}(i)$ $c(j, 16,16)=\operatorname{cupkmk}(i)$ $a(j, 9,1)=\operatorname{cuprek}(i)$ $a(j, 10,2)=\operatorname{cupuk}(i)$ $a(j, 11,3)=\operatorname{cupuk}(i)$ $a(j, 12,4)=\operatorname{cupuk}(i)$ $a(j, 13,5)=\operatorname{cupuk}(i)$ 


$$
\begin{aligned}
& a(j, 14,6)=\operatorname{cupuk}(i) \\
& a(j, 15,7)=\operatorname{cupuk}(i) \\
& a(j, 16,8)=\operatorname{cupuk}(i) \\
& a(j, 9,9)=1.0 \\
& a(j, 10,10)=1.0 \\
& a(j, 11,11)=1.0 \\
& a(j, 12,12)=1.0 \\
& a(j, 13,13)=1.0 \\
& a(j, 14,14)=1.0 \\
& a(j, 15,15)=1.0 \\
& a(j, 16,16)=1.0 \\
& b(j, 9,1, i)=\operatorname{cupupk}(i) \\
& b(j, 10,2, i)=\operatorname{cupupk}(i) \\
& b(j, 11,3, i)=\operatorname{cupupk}(i) \\
& b(j, 12,4, i)=\operatorname{cupupk}(i) \\
& b(j, 13,5, i)=\operatorname{cupupk}(i) \\
& b(j, 14,6, i)=\operatorname{cupupk}(i) \\
& b(j, 15,7, i)=\operatorname{cupupk}(i) \\
& b(j, 16,8, i)=\operatorname{cupupk}(i) \\
& b(j, 9,9, i)=\operatorname{cupkpk}(i) \\
& b(j, 10,10, i)=\operatorname{cupkpk}(i) \\
& b(j, 11,11, i)=\operatorname{cupkpk}(i) \\
& b(j, 12,12, i)=\operatorname{cupkpk}(i) \\
& b(j, 13,13, i)=\operatorname{cupkpk}(i) \\
& b(j, 14,14, i)=\operatorname{cupkpk}(i) \\
& b(j, 15,15, i)=\operatorname{cupkpk}(i) \\
& b(j, 16,16, i)=\operatorname{cupkpk}(i) \\
& e n d 10
\end{aligned}
$$$$
\text { do for } j=2, m-1
$$$$
\operatorname{rodzz}(j)=\operatorname{rhol}(i, j+1) \cdot \operatorname{sz3dv} l p(j)+\operatorname{rhol}(i, j-1) \cdot \operatorname{s23dvlm}(j)+\operatorname{rhol}(i, j)
$$$$
1 \cdot \operatorname{sid} 23 l(j)
$$$$
\operatorname{rodzm}(j)=\operatorname{rho}(i, j-1) \cdot s 13 m d u m 3(j)+\operatorname{rhol}(i, j-1) \cdot s 13 m d v l m(j)+\operatorname{rhol}(i, j
$$$$
1) \cdot s 13 m d v l(j)+r h o(i, j) \cdot s 13 m d v(j)
$$$$
\operatorname{rodzp}(j)=\operatorname{rho}(i, j+1) \cdot s 13 p d v p 3(j)+r h o l(i, j+1) \cdot s 13 p d v l p(j)+r h o l(i, j
$$ 
$1 \quad) \cdot s 13 p d v l(\jmath)+r h o(i, j) \cdot s 13 p d v(j)$

$\imath \cdot 1 d z z(j)=s 23 d \imath l m(\jmath) \cdot v 1 l(i, j-1)+s 23 d v l p(j) \cdot v 1 l(i, j+1)+s 23 d v l(\jmath) \cdot v$

$1 \quad l l(i, j)$

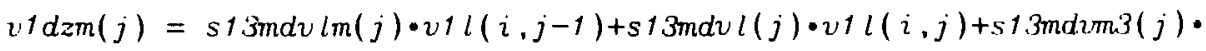

$1 \quad \imath 11(i, j-1)+s 13 m d v(j) \cdot v 1(i, j)$

$v 1 d z p(j)=s 13 p d v l p(j) \cdot v 1 l(i, j+1)+s 13 p d v l(j) \cdot v 1 l(i, j)+s 13 p d v p \cdot 3(j) \cdot$

$1 \quad v \cdot 1(i, j+1)+s 13 p d v(j) \cdot v 1(i, j)$

$v 2 d 2 z(j)=\operatorname{s} 23 d v l m(j) \cdot v 2 l(i, j-1)+\sin 2 v l p(j) \cdot v 2 l(i, j+1)+s 23 d v l(j) \cdot v$

$12 l(i, j)$

$v 2 d z m(j)=s 13 \pi d v l m(j) \cdot v 2 l(i, j-1)+s 13 m d v l(j) \cdot v 2 l(i, j)+s 13 m d v m 3(j) \cdot$

$1 \quad i 2(i, j-1)+s 1: 3 m d v(j) \cdot v 2(i, j)$

$v 2 d z p(j)=s 13 p d v l p(j) \cdot v 2 l(i, j+1)+s 13 p d v l(j) \cdot v 2 l(i, j)+s 13 \mu d v p 3(j) \cdot$

$1 \quad v 2(i, j+1)+s 13 p d v(j) \cdot v 2(i, j)$

$v 3 d 2 z(j)=\operatorname{sid} d v l m(j) \cdot v 3 l(i, j-1)+\sin d v l p(j) \cdot v 3 l(i, j+1)+\operatorname{s} 23 d v l(j) \cdot v$

$13 l(i, j)$

$v 3 d z m(j)=s 13 m d v l m(j) \cdot v 3 l(i, j-1)+s 13 m d v l(j) \cdot v \cdot 3 l(i, j)+s 13 m d v m 3(j) \cdot$

$1 \quad v 3(i, j-1)+s 13 \mathrm{md} v(j) \cdot v 3(i, j)$

$v 3 d z p(j)=s 13 p d v l p(j) \cdot v 3 l(i, j+1)+s 13 p d v l(j) \cdot v 3 l(i, j)+s 13 p d v p 3(j) \cdot$

$1 v 3(i, j+1)+s 13 p d v(j) \sim v 3(i, j)$

$t d 2 z(j)=s 23 d v \operatorname{lm}(j) \cdot t l(i, j-1)+s 23 d v l p(j) \cdot t l(i, j+1)+s 23 d v l(j) \cdot t l(i$

$1, j)$

$t d z m(j)=s 13 m d v l m(j) \cdot t l(i, j-1)+s 13 m d v l(j) \cdot t l(i, j)+s 13 m d v m 3(j) \cdot t(i$

$1 \quad, j-1)+s 13 m d v(j) \cdot t(i, j)$

$t d z p(j)=s 13 p d v l p(j) \cdot t l(i, j+1)+s 13 p d v l(j) \cdot t l(i, j)+s 13 p d v p 3(j) \cdot t(i$

$1, j+1)+s 13 p d v(j) \cdot t(i, j)$

$b 1 d 2 z(j)=b 1 l(i, j+1) \cdot s 23 d v l p(j)+b 1 l(i, j-1) \cdot s 23 d v l m(j)+b 1 l(i, j) \cdot s 2$

$13 d v l(j)$

$b 1 d z m(j)=b 1(i, j-1) \cdot s 13 m d v m 3(j)+b 1 l(i, j-1) \cdot s 13 m d v l m(j)+b i l(\imath, j) \cdot s$

$1 \quad 13 m d v l(j)+b i(i, j) \cdot s 13 m d v(j)$

$b 1 d z p(j)=b 1(i, j+1) \cdot s 13 p d v p 3(j)+61 l(i, j+1) \cdot s 13 p d v l p(j)+i 1 l(i, j) \cdot s$

$1 \quad 1.3 p d v l(j)+b 1(i, j) \cdot s 13 p d v(j)$

$b 2 d z z(j)=b 2 l(i, j+1) \cdot s 23 d v l p(j)+b 2 l(i, j-1) \cdot s 23 d v l m(j)+b 2 l(i, j) \cdot s 2$

$13 d v l(j)$

$b 2 d z m(j)=b 2(i, j-1) \cdot s 13 m d v m 3(j)+b 2 l(i, j-1) \cdot s 13 m d v l m(j)+b 2 l(2, j) \cdot s$ 
$b 2 d z p(j)=b 2(i, j+1) \cdot s 13 p d v p 3(j)+b 2 l(i, j+1) \cdot s 13 p d v l p(j)+b 2 l(i, j) \cdot s$

$1 \quad 13 p d v l(\jmath)+b 2(i, j) \cdot s 13 p d v(j)$

$b 3 d 2 z(j)=b 3 l(i, j+1) \cdot s 23 d v l p(j)+b 3 l(i, j-1) \cdot s 23 d v l m(j)+b 3 l(i, j) \cdot s 2$

$13 \mathrm{~d} v l(j)$

$b 3 d z m(j)=b 3(i, j-1) \cdot s 13 m d v m 3(j)+b 3 l(i, j-1) \cdot s 13 m d v l m(j)+b 3 l(i, j) \cdot s$

$113 m d v l(j)+b 3(i, j) \cdot s 13 m d v(j)$

$b 3 d z p(j)=b 3(i, j+1) \cdot s 13 p d v p 3(j)+b 3 l(i, j+1) * s 13 p d v l p(j)+b 3 l(i, j) \cdot s$

$1 \quad 13 p d v l(j)+b 3(i, j) \cdot s 13 p d v(j)$

$\operatorname{opt1}(j)=\operatorname{cumgp}(s 11 \mathrm{mdum}(i), 0.0,-v 1(i, j))$

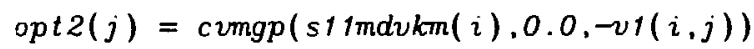

$\operatorname{opt} 3(j)=i / p r h o(i, j)$

opt $4(j)=\operatorname{opt} 1(j) \cdot t i m f a c \cdot v 1(i, j) \cdot x x 8 \cdot o p t 3(j)$

$o p t 5(j)=\operatorname{opt} 2(j) \cdot t i m f a c \cdot v 1(i, j) \cdot x x 8 \cdot o p t 3(j)$

opt $6(j)=-b 1(i, j) \cdot c u m g p(s 11 \mathrm{mdum}(i), 0.0, b 1(i, j)) \cdot t i m f a c * x x 12 \cdot c p t 3($

$1 j)$

$o p t 7(j)=-b 1(i, j) \cdot c u m g p(s 11 m d v k m(i), 0.0, b 1(i, j)) \cdot t i m f a c \cdot x x 12 \cdot o p t 3$

$1 \quad(j)$

opt $8(j)=1 / r(i)$

$\operatorname{opt} 9(j)=b 1(i, j) \cdot \operatorname{cumgp}(s 11 \mathrm{mdum}(i), 0.0,-b 1(i, j)) \cdot \operatorname{timfac} \cdot x x 16$

opt $10(j)=\operatorname{cumgp}(-s 11 m d v m(i), 0.0, v 1(i, j)) \cdot \operatorname{timfac} \cdot v 1(i, j) \cdot x x 16$

opt11 $(j)=b 1(i, j) \cdot \operatorname{cumgp}(s 11 \mathrm{mdvkm}(i), 0.0,-b 1(i, j)) \cdot \operatorname{timfac} \cdot x x 16$

opt12(j) $=\operatorname{crmgp}(-s 11 m d v k m(i), 0.0, v 1(i, j)) \cdot \operatorname{timfac} \cdot v 1(i, j) \cdot x x 16$

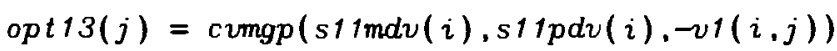

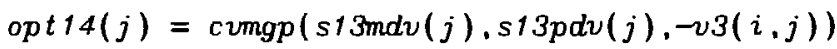

opt $15(j)=$ opt $14(j) \cdot v 3(i, j)$

opt $16(j)=\operatorname{rho}(i, j) \cdot \operatorname{opt} g(j)$

$\operatorname{opt17}(j)=c u m g p(\operatorname{rodrm}(j), \operatorname{rodrp}(j),-v 1(i, j))$

opt 18(j) $=\operatorname{cumgp}(\operatorname{rodzm}(j), \operatorname{rodzp}(j),-v 3(i, j))$

opt $19(j)=\operatorname{cvmgp}(s 11 \operatorname{mdvk}(i), s 11 p d v k(i),-v 1(i, j))$

opt $20(j)=v 2(i, j) \cdot \cdot 2 \cdot x x 8-b 2(i, j) \cdot \cdot 2 \cdot x \times 12$

opt $21(j)=1 /(r(i) \cdot r h o(i, j) \cdot \cdot 2)$

$\operatorname{opt} 22(j)=\operatorname{opt} 13(j) \cdot v 1(i, j)$

$\operatorname{opt} 23(j)=\operatorname{cvmgp}(v 1 d r m(j), v 1 d r p(j),-v 1(i, j))$ 


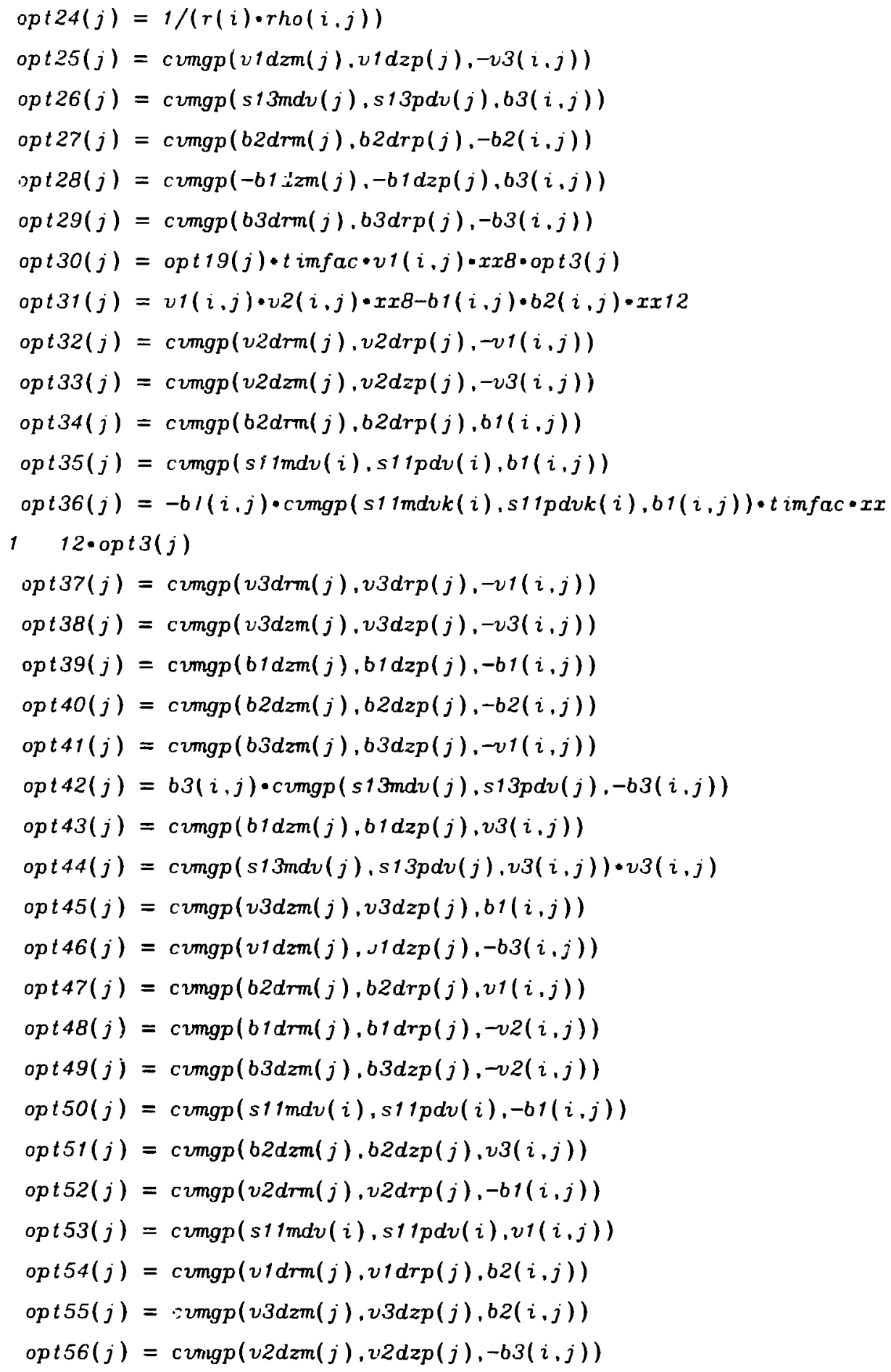




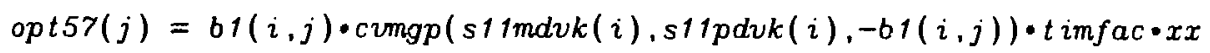

$1 \quad 16$

$\operatorname{opt58}(j)=\operatorname{cvmgp}(-s 11 m d v k(i),-s 11 p d v k(i), v 1(i, j)) \cdot t i m f a c \cdot v 1(i, j) \cdot x$

$1 \quad x 16$

$\operatorname{opt59}(j)=\operatorname{cumgp}(b 3 d r m(j), b 3 d r p(j), v 1(i, j))$

$\operatorname{opt} 60(j)=\operatorname{cvmgp}(b 1 d r m(j), b 1 d r p(j),-v 3(i, j))$

$\operatorname{opt61}(j)=\operatorname{cumgp}(v 3 d r m(j), v 3 d r p(j),-b 1(i, j))$

opt62(j) $=\operatorname{cumgp}(v 1 d r m(j), v 1 d r p(j), b 3(i, j))$

opt63(j) $=\operatorname{cumgp}(0.0, \operatorname{s11pdvp}(i),-v 1(i, j))$

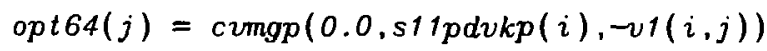

opt $65(j)=\operatorname{opt} 63(j) \cdot t i m f a c * v 1(i, j) \cdot x x 8 \cdot o p t 3(j)$

opt $66(j)=\operatorname{opt} 64(j) \cdot t i m f a c \cdot v 1(i, j) \cdot x x 8 \cdot o p t 3(j)$

$\operatorname{opt} 67(j)=\operatorname{cumgp}(0.0,-s 11 p d v p(i), b 1(i, j)) \cdot b 1(i, j) \cdot t i m f a c \cdot x x 12 \cdot o p t 3$

$1 \quad(j)$

$o p t 68(j)=\operatorname{cvmgp}(0.0,-s 11 p d v k p(i), b 1(i, j)) \cdot b 1(i, j) \cdot t i m f a c \cdot x x 12 \cdot o p t$

$13(j)$

$o p t 69(j)=\operatorname{cumgp}(0.0, s 11 p d v p(i),-b 1(i, j)) \cdot b 1(i, j) \cdot t i m f a c * x \times 16$

$o p t 70(j)=\operatorname{cumgp}(0.0,-s 11 p d v p(i), v 1(i, j)) \cdot \operatorname{timfac} \cdot v 1(i, j) \cdot x x 16$

$\operatorname{opt71}(j)=\operatorname{cumgp}(0.0, s 11 p d v k p(i),-b 1(i, j)) \cdot b 1(i, j) \cdot t i m f a c \cdot x x 16$

$\operatorname{opt72}(j)=\operatorname{cvmgp}(0.0,-s 11 p d v k p(i), v 1(i, j)) \cdot t i m f a c \cdot v 1(i, j) \cdot x x 16$

$c(j, 1,1)=$ opt $1(j) \cdot t$ imfac $* v 1(i, j) \cdot x x 5$

$c(j, 1,9)=\operatorname{opt} 2(j) \cdot t$ imfac*v1 $(i, j) \cdot x x 5$

$c(j, 2,2)=\operatorname{opt} 4(j)$

$c(j, 2,7)=b 2(i, j) \cdot c u m g p(s 11 \mathrm{mdum}(i), 0.0,-b 2(i, j)) \cdot \operatorname{timfac} \cdot x x 12 \cdot o p t 3$

$1 \quad(j)$

$c(j, 2,8)=b 3(i, j) \cdot c v m g p(s 11 m d v m(i), 0.0,-b 3(i, j)) \cdot t i m f a c \cdot x x 12 \cdot o p t 3$

$1 \quad(j)$

$c(j, 2,10)=\operatorname{opt} 5(j)$

$c(j, 2,15)=b 2(i, j) \cdot \operatorname{cumgp}(s 11 m d v k m(i), 0.0,-b 2(i, j)) \cdot t i m f a c \cdot x x 12 \cdot o p$

$1 \quad t 3(j)$

$c(j, 2,16)=b 3(i, j) \cdot \operatorname{cvmgp}(s 11 m d v k m(i), 0,0,-b 3(i, j)) \cdot t i m f a c \cdot x x 12 \cdot o p$

$1 \quad t 3(j)$

$c(j, 3,3)=\operatorname{opt} 4(j)$

$c(j, 3,7)=\operatorname{opt} 6(j)$ 


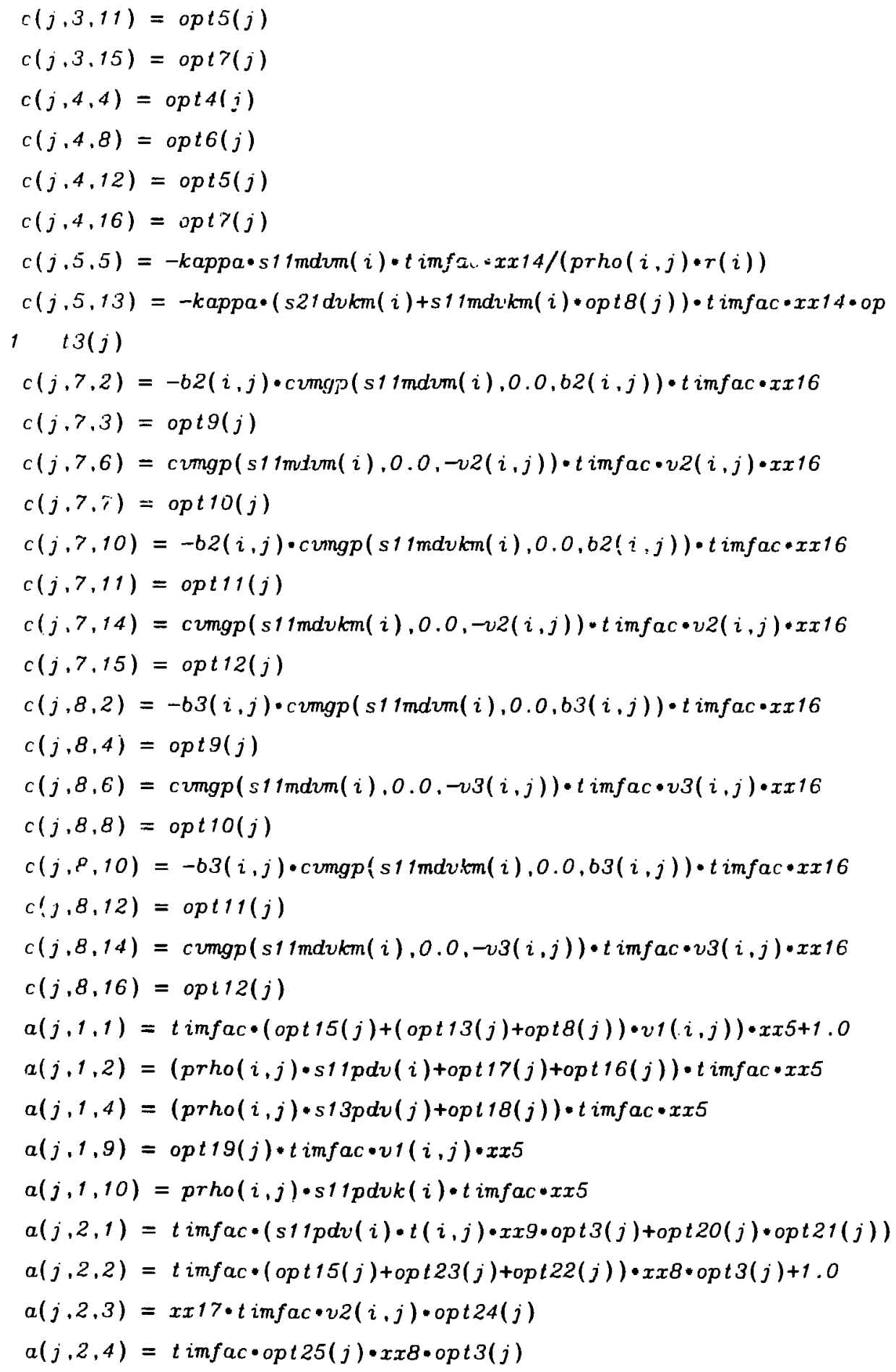




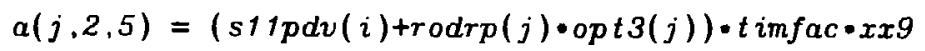

$a(j, 2,6)=-b 3(i, j) \cdot o p t 26(j) \cdot t i m f a c \cdot x x 12 \cdot o p t 3(j)$

$a(j, 2,7)=(b 2(i, j) \cdot(\operatorname{cumgp}(s 11 m d v(i), s 11 p d v(i),-b 2(i, j)) \cdot$ opt $3(j)+2$

$1 \quad .0 \cdot o p t 24(j))+o p t 27(j) \cdot o p t 3(j)) \cdot t i m f a c \cdot x x 12$

$a(j, 2,8)=(b 3(i, j) \cdot \operatorname{cumgp}(s 11 m d v(i), s 11 p d v(i),-b 3(i, j))+o p t 29(j)+o$

$1 p t 28(j)) \cdot t i m f a c \cdot x x 12 \cdot o p t 3(j)$

$a(j, 2,9)=s 11 p d v k(i) \cdot t(i, j) \cdot t i m f a c \cdot x x 9 \cdot o p t 3(j)$

$a(j, 2,10)=$ opt $30(j)$

$a(j, 2,13)=s 11 p d v k(i) \cdot t i m f a c \cdot x x 9$

$\alpha(j, 2,15)=b 2(i, j) \cdot c v m g p(s 11 m d v k(i), s 11 p d v k(i),-b 2(i, j)) \cdot t i m f a c \cdot x$

$1 \quad x 12 \cdot \operatorname{opt} 3(j)$

$a(j, 2,16)=b 3(i, j) \cdot c u m g p(s 11 m d v k(i), \operatorname{sipdvk}(i),-b 3(i, j)) \cdot t i m f a c \cdot x$

$1 \quad x 12 \cdot o p t 3(j)$

$a(j, 3,1)=-t$ imfac opt $31(j) \cdot$ opt $21(j)$

$a(j, 3,2)=\operatorname{timfac} \cdot($ opt $32(j) \cdot o p t 3(j)+v 2(i \cdot j) \cdot o p t 24(j)) \cdot x x 8$

$a(j, 3,3)=t$ imfac $\cdot($ oyt $14(j) \cdot v 3(i, j) \cdot$ opt $3(j)+($ opt $13(j) \cdot$ opt $3(j)+o p t 2$

$14(j)) \cdot v 1(i, j)) \cdot x x 8+1.0$

$a(j, 3,4)=t$ imfac opt $33(j) \cdot x x 8 \cdot o p t 3(j)$

$a(j, 3,6)=-(b 2(i, j) \cdot o p t 24(j)+o p t 34(j) \cdot o p t 3(j)) \cdot t i m f a c * x x 12$

$a(j, 3,7)=-(b 3(i, j) \cdot$ opt $26(j) \cdot$ opt $3(j)+b 1(i, j) \cdot($ opt $35(j) \cdot$ opt $3(j)+o p$

$1 t 24(j))) \cdot t i m f a c \cdot x x 12$

$a(j, 3,8)=c u m g p(-b 2 d z m(j),-b 2 d z p(j), b 3(i, j)) \cdot t i m f a c \cdot x x 12 \cdot o p t 3(j)$

$a(j, 3,11)=$ opt $30(j)$

$a(j, 3,15)=$ opt $36(j)$

$a(j, 4,1)=s 13 p d v(j) \cdot t(i, j) \cdot t$ imfac $\cdot x x 9 \cdot o p t 3(j)$

$a(j, 4,2)=t$ imfac opt $37(j) \cdot x x 8 \cdot o p t 3(j)$

$a(j, 4,4)=t$ imfac $\cdot($ opt $38(j)+o p t 15(j)+o p t 22(j)) \cdot x x 8 \cdot o p t 3(j)+1.0$

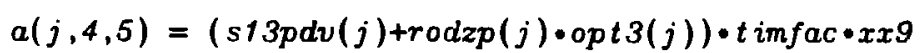

$a(j, 4,6)=(b 1(i, j) \cdot c 2 m g p(s 13 m d v(j), s 13 p d v(j),-b 1(i, j))+c v m g p(-b 3 d$

$1 \quad r m(j),-b 3 d r p(j), b 1(i, j))+o p t 39(j)) \cdot t i m f a c \cdot x x^{1} 2 \cdot o p t 3(j)$

$a(j, 4,7)=(b 2(i, j) \cdot c v m g p(s 13 \operatorname{mdv}(j), s 13 p d v(j),-b 2(i, j))+o p t 4 O(j)) \cdot$

$1 \quad t$ imfac $\cdot x x 12 \cdot o p t 3(j)$

$a(j, 4,8)=-b 1(i, j) \cdot o p t 35(j) \cdot t i m f a c \cdot x x 12 \cdot o p i 3(j)$

$a(j, 4,12)=$ opt $30(j)$ 


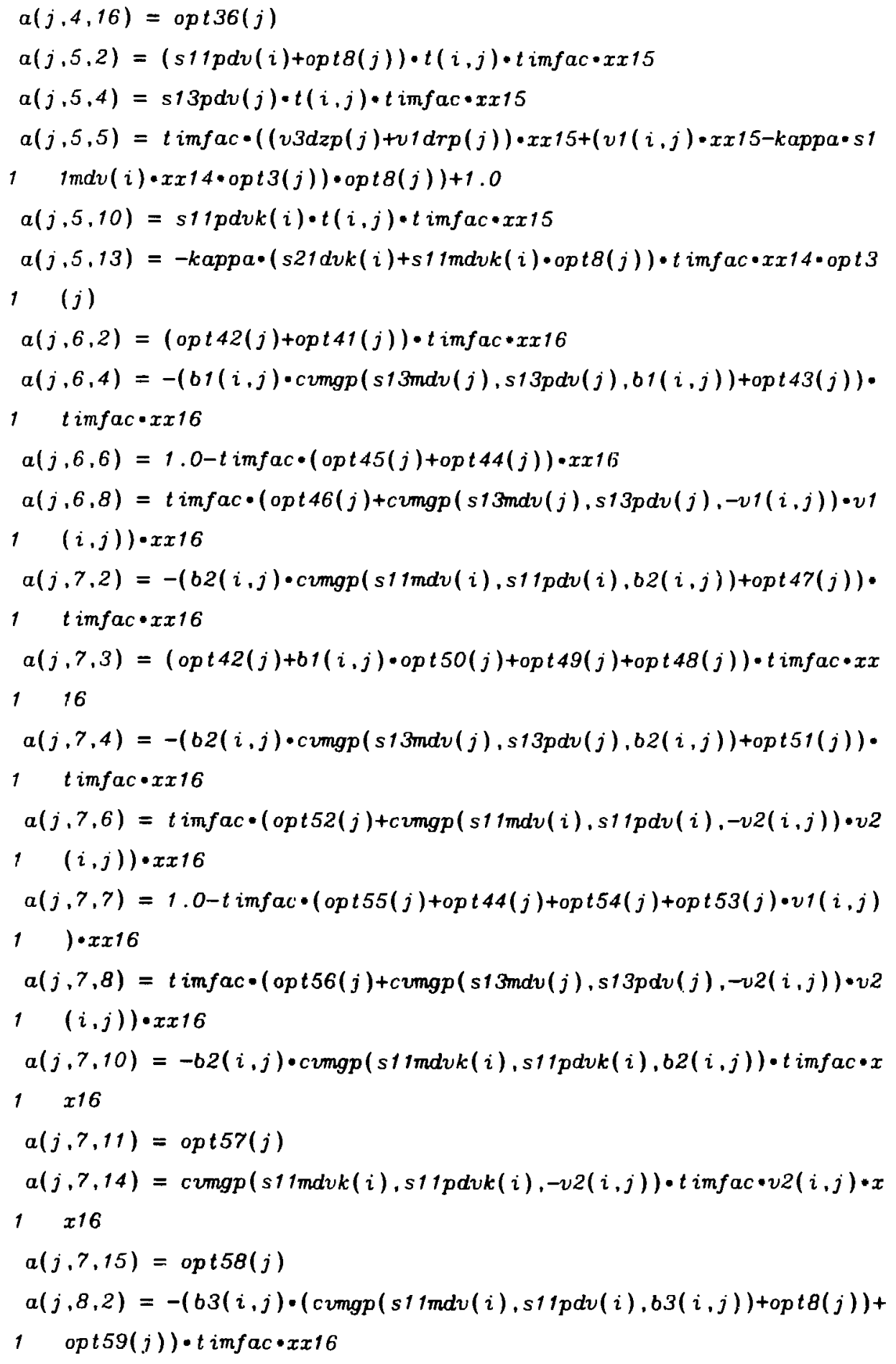




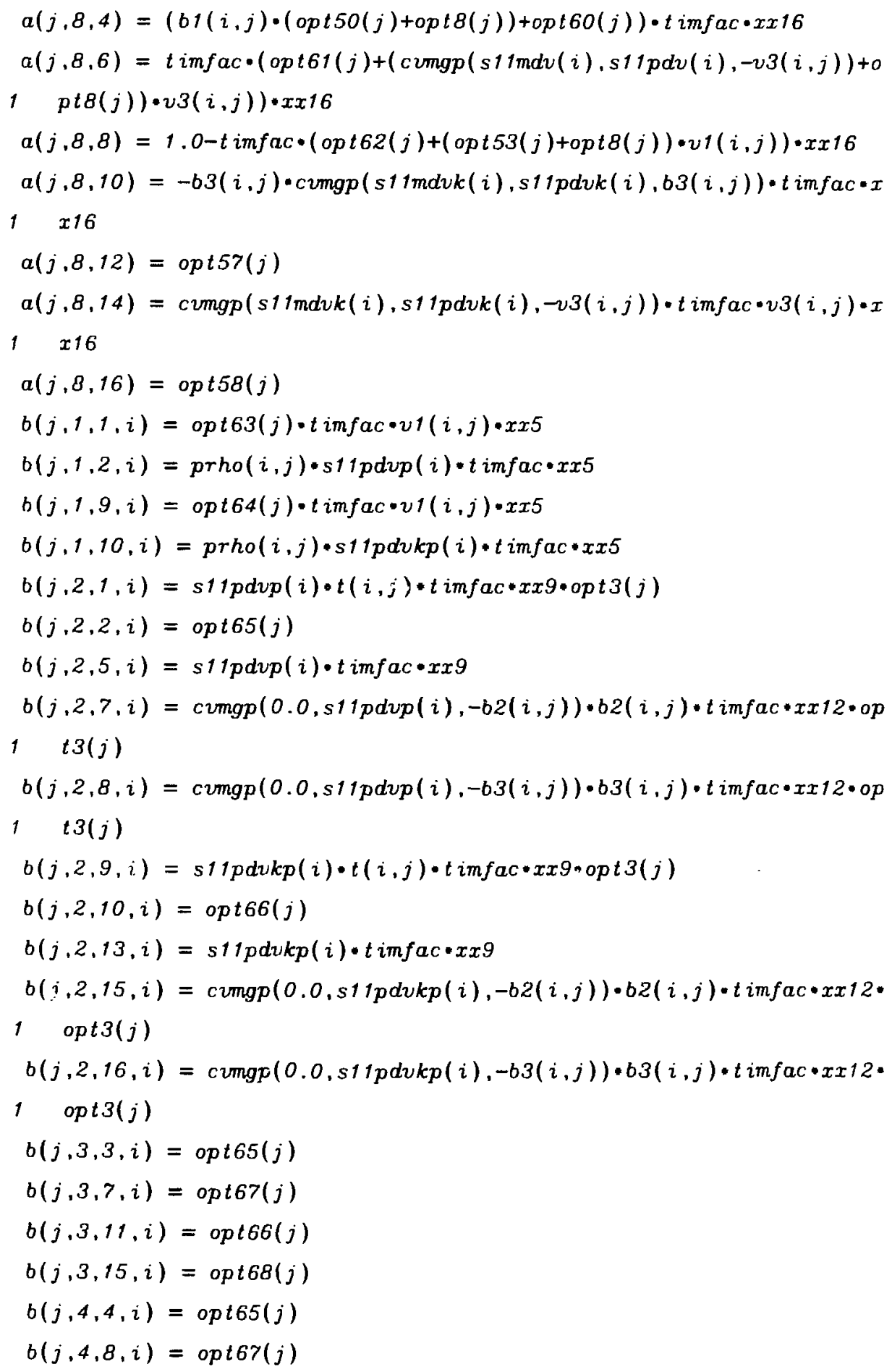







,j)-histv3(i,j)

$\operatorname{rhs}(j, 5, i)=-t i m f a c \cdot(t(i, j) \cdot(v 3 d z p(j)+v 1 d r p(j)+\imath 1(i, j) \cdot$ opt $8(j)) \cdot x$

$1 \quad x 15-k a p p a \cdot(t d r m(j) \cdot o p t 8(j)+t d 2 z(j)+t d 2 r(j)) \cdot x x 14 \cdot o p t 3(j))-t(i, j$

$2 \quad)-h i s t(i, j)$

$\operatorname{rhs}(j, 6, i)=t$ imfac $(b 1(i, j) \cdot$ opt $45(j)+o p t 43(j) \cdot v 3(i, j)-(b 3(i, j) * o p$

$1 \quad t 46(j)+o p t 41(j) \cdot v 1(i, j))) \cdot x x 16-h i s t b 1(i, j)-b 1(i, j)$

$\operatorname{rhs}(j, 7, i)=t i m f a c \cdot(b 2(i, j) \cdot o p t 55(j)+o p t 51(j) \cdot v 3(i, j)-(b 3(i, j) \cdot o p$

$1 \quad t 56(j)+b 1(i, j) \cdot o p t 52(j)+(o p t 49(j)+o p t 48(j)) \cdot v 2(i, j))+b 2(i, j) \cdot o p$

$2 \quad t 54(j)+o p t 47(j) \cdot v 1(i, j)) \cdot x x 16-h i s t b 2(i, j)-b 2(i, j)$

$\operatorname{rhs}(j, 8, i)=-t i m f a c \cdot(b 1(i, j) \cdot o p t 61(j)+(b 1(i, j) \cdot v 3(i, j)-b 3(i, j) \cdot v 1$

$1(i, j)) \cdot \operatorname{opt} 8(j)+o p t 60(j) \cdot v 3(i, j)-(b 3(i, j) \cdot \operatorname{opt} 62(j)+o p t 59(j) \cdot v 1(i$

$2 \quad, j)) \cdot x x 16-h i s t b 3(i, j)-b 3(i, j)$

enddo

Next, the top boundary segmeni is described by "gcook;middl1 end":

$\operatorname{rodzm}(m)=\operatorname{rho}(i, m-1)+s 13 m d v m 3(m)+\operatorname{rhol}(i, m-1) \cdot s 13 m d v l m(m)+r h o l(i, m$

$1 \quad) \cdot s 13 m d v l(m)+r h o(i, m) \cdot s 13 m d v(m)$

$\operatorname{rod} 2 z m(i)=\operatorname{coelnm} 2 \cdot \operatorname{rhol}(i, m-2)+\operatorname{coelnm} \cdot \operatorname{rhol}(i, m-1)+\operatorname{coeln} \cdot \operatorname{rhol}(i, m)$ $v 1 d z m(m)=s 13 m d v l m(m) \cdot v 1 l(i, m-1)+s 13 m d v l(m) \cdot v 1 l(i, m)+s 13 m d v m 3(m) \cdot$

$1 \quad v i(i, m-1)+s 13 m d v(m) \cdot v 1(i, m)$

$v 1 d 2 z m(i)=$ coelnm2*v1l $(i, m-2)+\operatorname{coelnan} \cdot 11 l(i, m-1)+\operatorname{coe} \ln \cdot v 1 l(i, m)$ $v 2 d z m(m)=s 13 n d v l m(m) \cdot v 2 l(i, m-1)+s 13 m d v l(m) \cdot v 2 l(i, m)+s 13 m d v m 3(m) \cdot$

$1 \quad v 2(i, m-1)+s 13 m d v(m) \cdot v 2(i, m)$

$v 2 d 2 z m(i)=\operatorname{coe} l n m 2 \cdot v 2 l(i, m-2)+\operatorname{coe} l n m \cdot v 2 l(i, m-1)+\operatorname{coe} \ln \cdot v 2 l(i, m)$ $v 3 d z m(m)=s 13 m d v l m(m) \cdot v 3 l(i, m-1)+s 13 m d v l(m) \cdot v 3 l(i, m)+s 13 m d v m 3(m) \cdot$

$1 \quad v 3(i, m-1)+s 13 m d v(m) \cdot v 3(i, m)$

$v 3 d 2 z m(i)=\operatorname{coe} l n m 2 \cdot v 3 l(i, m-2)+\operatorname{coe} l n m \cdot v 3 l(i, m-1)+\operatorname{coe} \ln \cdot v 3 l(i, m)$

$t d z m(m)=s 13 m d v l m(m) \cdot t l(i, m-1)+s 13 m d v l(m) \cdot t l(i, m)+s 13 m d v m 3(m) \cdot t(i$

$1, m-1)+s 13 d v(m) \cdot t(i, m)$

$t d Z_{2 m}(i)=\operatorname{coe} l n m 2 \cdot t l(i, m-2)+\operatorname{coe} l$ rom $t l(i, m-1)+\operatorname{coe} l n \cdot t l(i, m)$

$b 1 d z m(m)=b 1(i, m-1) \cdot s 13 m d v m 3(m)+b 1 l(i, m-1) \cdot s 13$ mdv $l m(m)+b 1 l(i, m) \cdot s$ 
$b 1 d 2 z m(i)=b 1 l(i, m-2) \cdot \operatorname{coe} \ln m 2+b 1 l(i, m-1) \cdot \operatorname{coeln} m+b 1 l(i, m) \cdot \operatorname{coeln}$ $b 2 d z m(m)=b 2(i, m-1) \cdot s 13 m d v m 3(m)+b 2 l(i, m-1) \cdot s 13 m d v l m(m)+b 2 l(i, m) \cdot s$

$113 m d v l(m)+b 2(i, m) \cdot s 13 m d v(m)$

$b 2 d 2 z m(i)=b 2 l(i, m-2) \cdot c o e l n m 2+b 2 l(i, m-1) \cdot \operatorname{coe} l n m+b 2 l(i, m) \cdot$ coeln $b 3 d z m(m)=b 3(i, m-1) \cdot s 13 m d v m 3(m)+b 3 l(i, m-1) \cdot s 13 m d v l m(m)+b 3 l(i, m) \cdot s$

$113 m d v l(m)+b 3(i, m) * s 13 m d v(m)$

$b 3 d 2 z m(i)=b 3 l(i, m-2) \cdot \operatorname{coe} l n m 2+b 3 l(i, m-1) \cdot \operatorname{coeln} \ln +b 3 l(i, m) \cdot \operatorname{coeln}$ $a(m, 1,1)=1.0$

$a(m, 2,2)=1.0$

$a(m, 3,3)=1.0$

$a(m, 4.4)=1.0$

$a(m, 5,5)=1.0$

$a(m, 6,6)=1.0$

$a(m, 7,7)=1.0$

$a(m, 8,8)=1.0$

$\operatorname{rhs}(m, 1, i)=\operatorname{rhowall} l-r h o(i, m)$

$\operatorname{rhs}(m, 2, i)=v$ tual $l-v 1(i, m)$

$\operatorname{rhs}(m, 3, i)=v$ Zual $l-v 2(i, m)$

$\operatorname{rhs}(m, 4, i)=v 3 w a l l-v 3(i, m)$

$\operatorname{rhs}(m, 5, i)=\operatorname{twa} l l-t(i, m)$

$\operatorname{rhs}(m, 6, i)=b$ twa $l l-b l(i, m)$

$\operatorname{rhs}(m, 7, i)=$ bzwal $l-b 2 .(i, m)$

$\operatorname{rhs}(m, 8, i)=63 i i \bar{z} l i-33(i, m)$

For the bottom boundary, the FORTRAN results are in "gcook;middll start":

$\operatorname{rodzp}(1)=\operatorname{rho}(i, 2) \cdot s 13 p d v p 3(1)+\operatorname{rhol}(i, 2) \cdot s 13 p d v l p(1)+\operatorname{rho} l(i, 1) \cdot s 1$

$13 p d u l(1)+r h o(i, 1) \cdot s 13 p d v(1)$

$\operatorname{rod} 2 z p(i)=\operatorname{coel} 3 \cdot \operatorname{rhol}(i, 3)+\operatorname{coe} l 2 \cdot \operatorname{rhol}(i, 2)+\operatorname{coel} l \cdot \operatorname{rhol}(i, 1)$

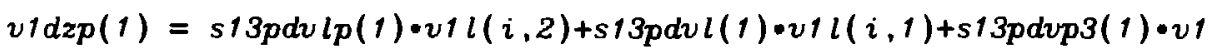




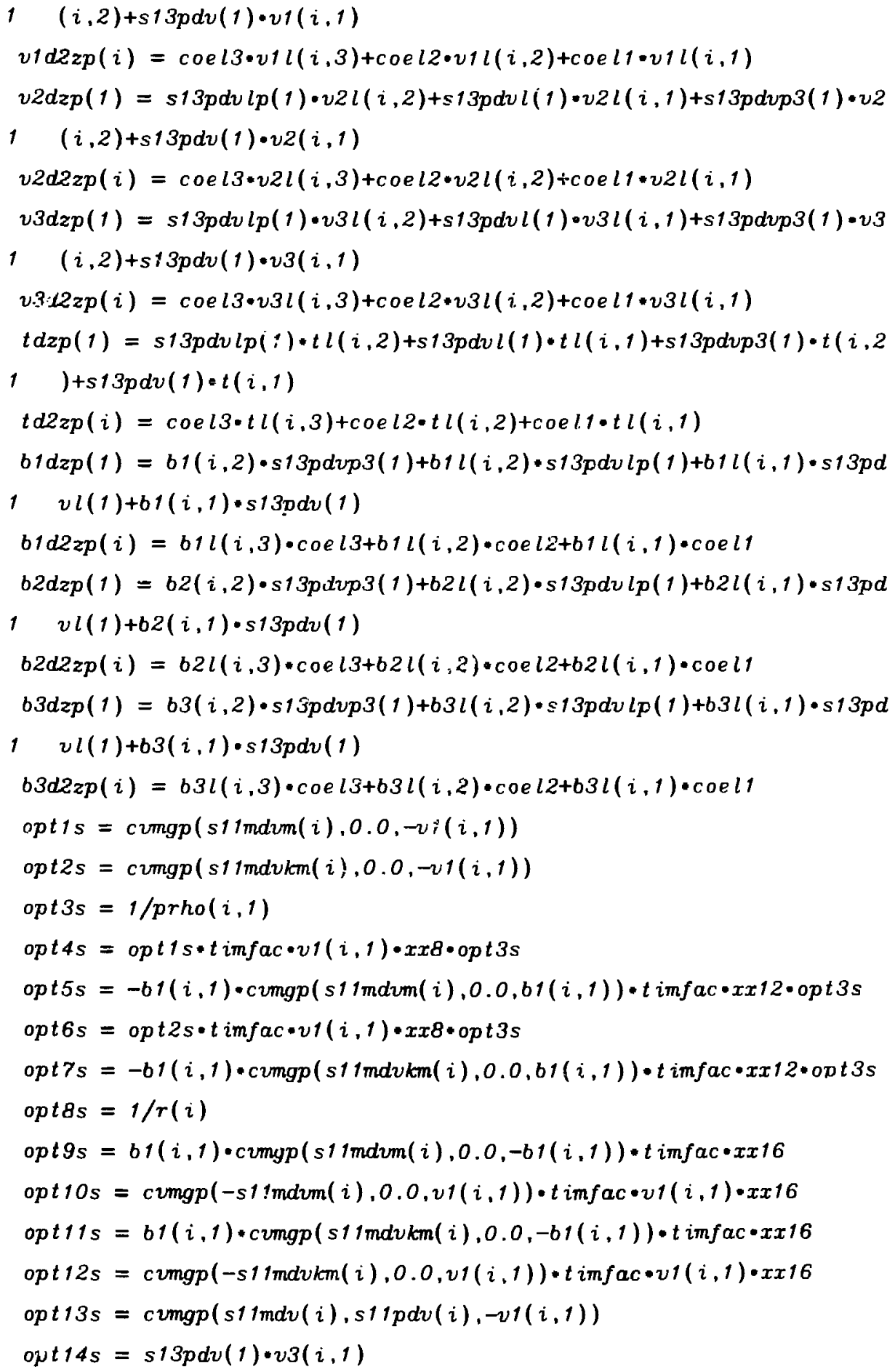




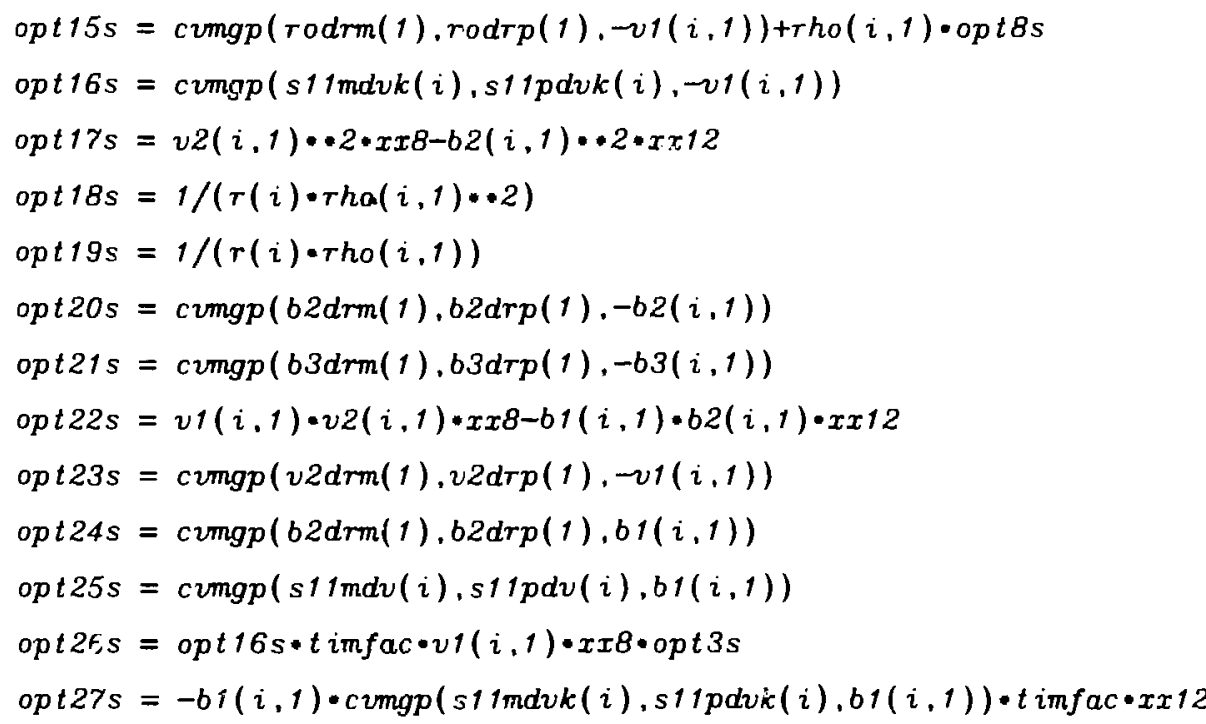

1 - opt3s

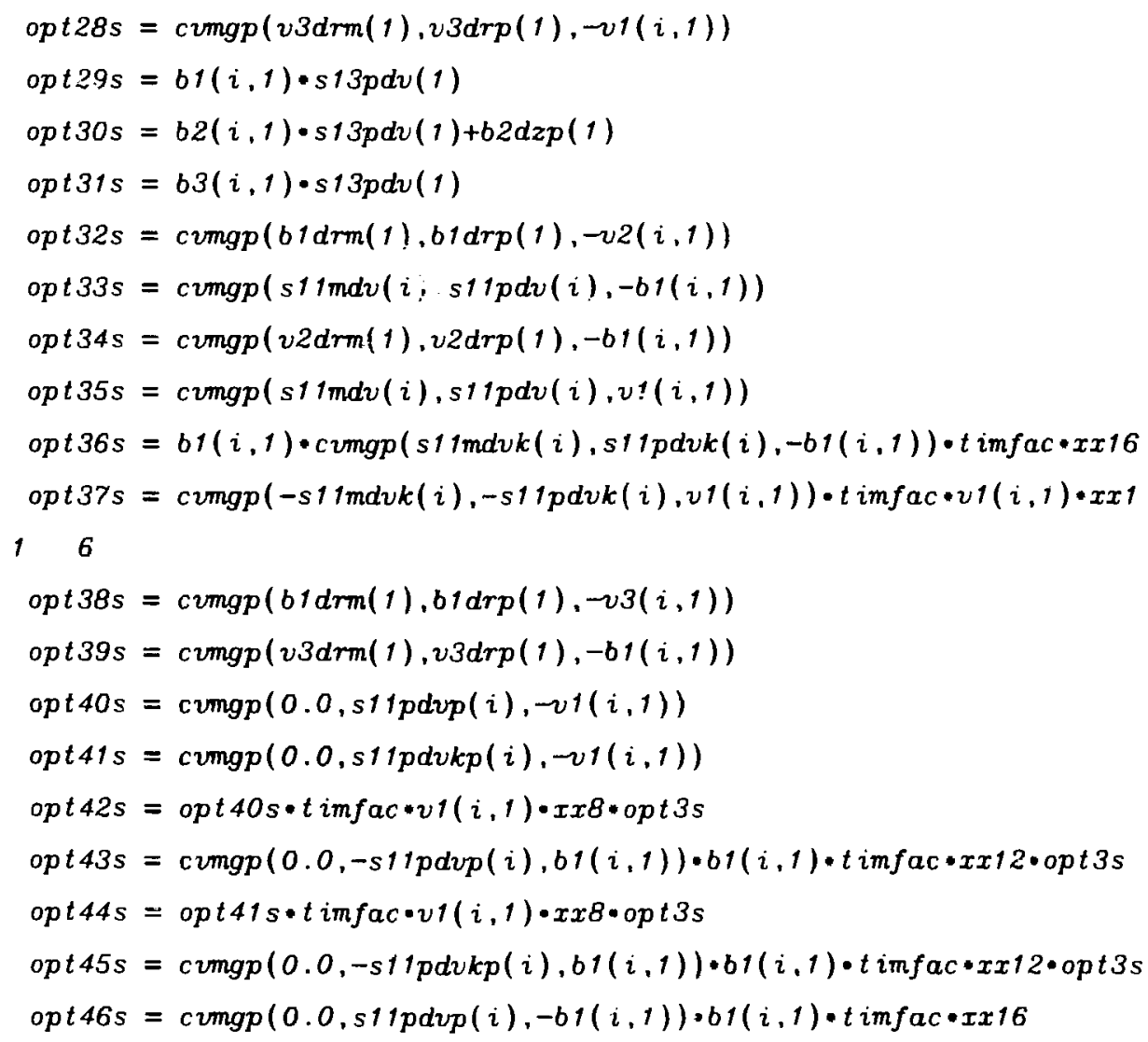


$\operatorname{opt} 47 \mathrm{~s}=\operatorname{cumgp}(0.0,-\mathrm{s} 11 \operatorname{pdvp}(i), v 1(i, 1)) \cdot t i m f a c \cdot v 1(i, 1) \cdot x x 16$

$\operatorname{cpt} 48 s=\operatorname{cvmgp}(0.0, s 11 p d v k p(i),-b 1(i, 1)) \cdot b 1(i, 1) \cdot t i m f a c * x x 16$

opt $49 s=\operatorname{cvmgp}(0.0,-s 11 p d v k p(i), v 1(i, 1)) \cdot t i m f a c=v 1(i, 1) \cdot x x 16$

$c(1,1,1)=$ opt $1 \mathrm{~s} \cdot \operatorname{timfac} \cdot v 1(i .1) \cdot x x 5$

$c(1,1,9)=$ opt2s*timfac*v1 $(i, 1) \cdot x x 5$

$c(1,2,7)=b 2(i, 1) \cdot c v m g p(\operatorname{simdvm}(i), 0.0,-b 2(i, 1)) \cdot t i m f a c \cdot x x 12 \cdot o p t 3$

$1 \mathrm{~s}$

$c(1,2,8)=b 3(i, 1) \cdot c u m g p(s 11 m d v m(i), 0.0,-b 3(i, 1)) \cdot t i m f a c \cdot x x 12 \cdot o p t 3$

$1 S$

$c(1,2,15)=b 2(i, 1) \cdot \operatorname{cumgp}(s 11 m d v k m(i), 0.0,-b 2(i, 1)) \cdot t i m f a c \cdot x x 12 \cdot o p$

1 t3s

$c(1,2,16)=b 3(i, 1) \cdot c u m g p(s 11 m d v k m(i), 0.0,-b 3(i, i)) \cdot t$ imfac $\bullet x x 12 \cdot o p$

1 t3̣s

$c(1,3,3)=$ opt $t s$

$c(1,3,7)=$ opt5s

$c(1,3,11)=$ opt $6 s$

$c(1,3,15)=$ opt7s

$c(1,4,4)=\operatorname{opt} 4 \mathrm{~s}$

$c(1,4,8)=$ opt5s

$c(1,4,12)=$ opt $6 \mathrm{~s}$

$c(1,4,16)=$ opt7s

$c(1,5,5)=-k a p p a \cdot s 11$ mdvm $(i) \cdot t i m f a c \cdot x x 14 /(\operatorname{prho}(i, 1) \cdot r(i))$

$c(1,5,13)=-k a p p a \cdot(s 21 d v k m(i)+s 11 m d v k m(i) * o p t 8 s) \cdot t i m f a c \cdot x x 14 \cdot o p t 3$

15

$c(1,7,3)=\operatorname{opt} 9 \mathrm{~s}$

$c(i, 7,6)=\operatorname{cumgp}(\operatorname{silmdvm}(i), 0.0,-v 2(i, 1)) \cdot t i m f a c \cdot v 2(i, 1) \cdot x x 16$

$c(1,7,7)=$ opt10s

$c(1,7,11)=$ opt11s

$c(1,7,14)=\operatorname{cvmgp}(s 11 m a v k m(i), 0.0,-v 2(i, 1)) \cdot \operatorname{timfac} \cdot v 2(i, 1) \cdot x x 16$

$c(1,7,15)=$ opt12s

$c(1,8,4)=$ opt $9 \mathrm{~s}$

$c(1,8,6)=\operatorname{cumgp}(\operatorname{silmdvm}(i), 0.0,-v 3(i, 1)) \cdot t i m f a c \cdot v 3(i, 1) \cdot x x 16$

$c(1,8,8)=$ opt $10 \mathrm{~s}$

$c(1,8,12)=o p t 11 \mathrm{~s}$ 


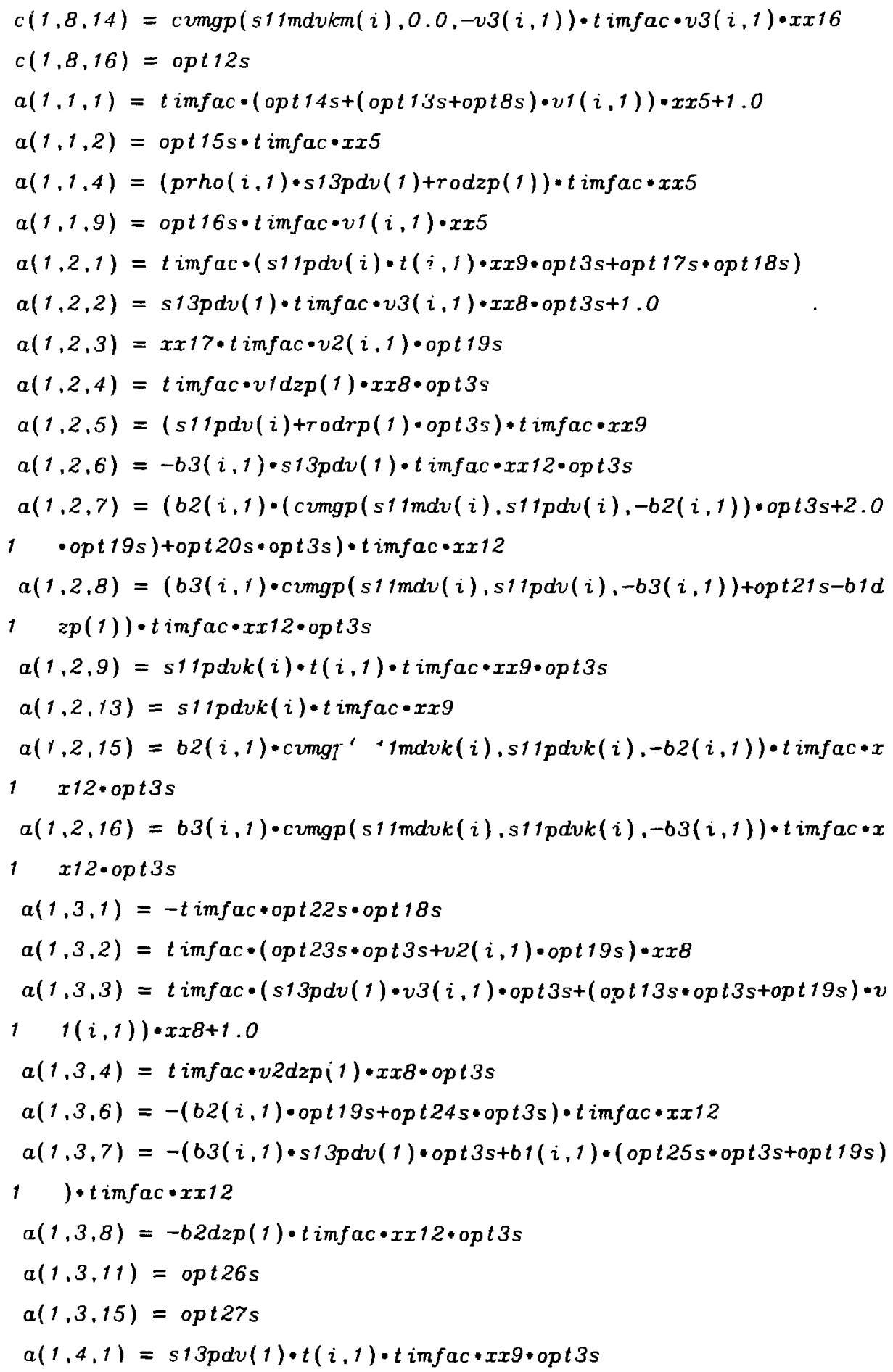




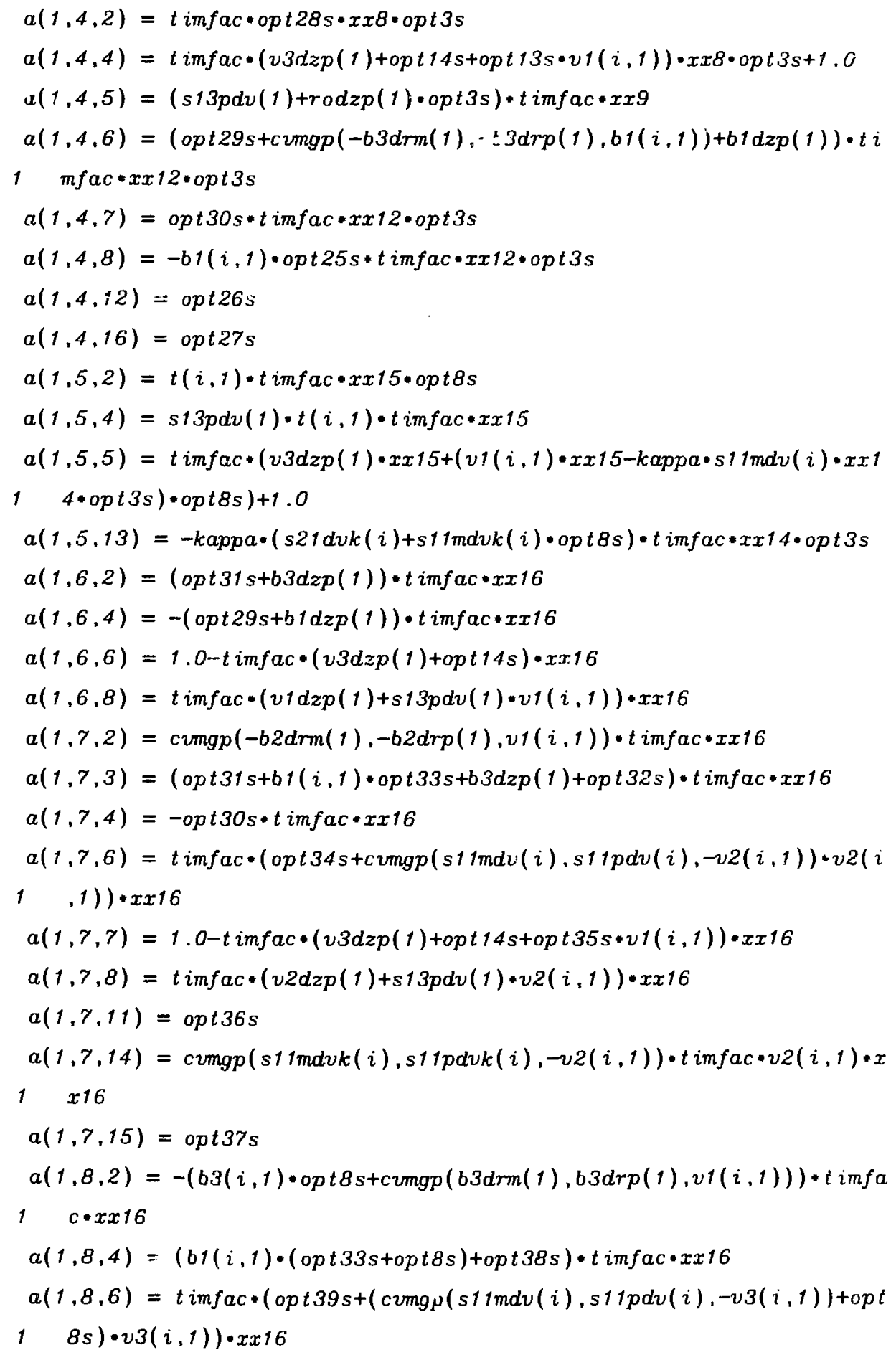




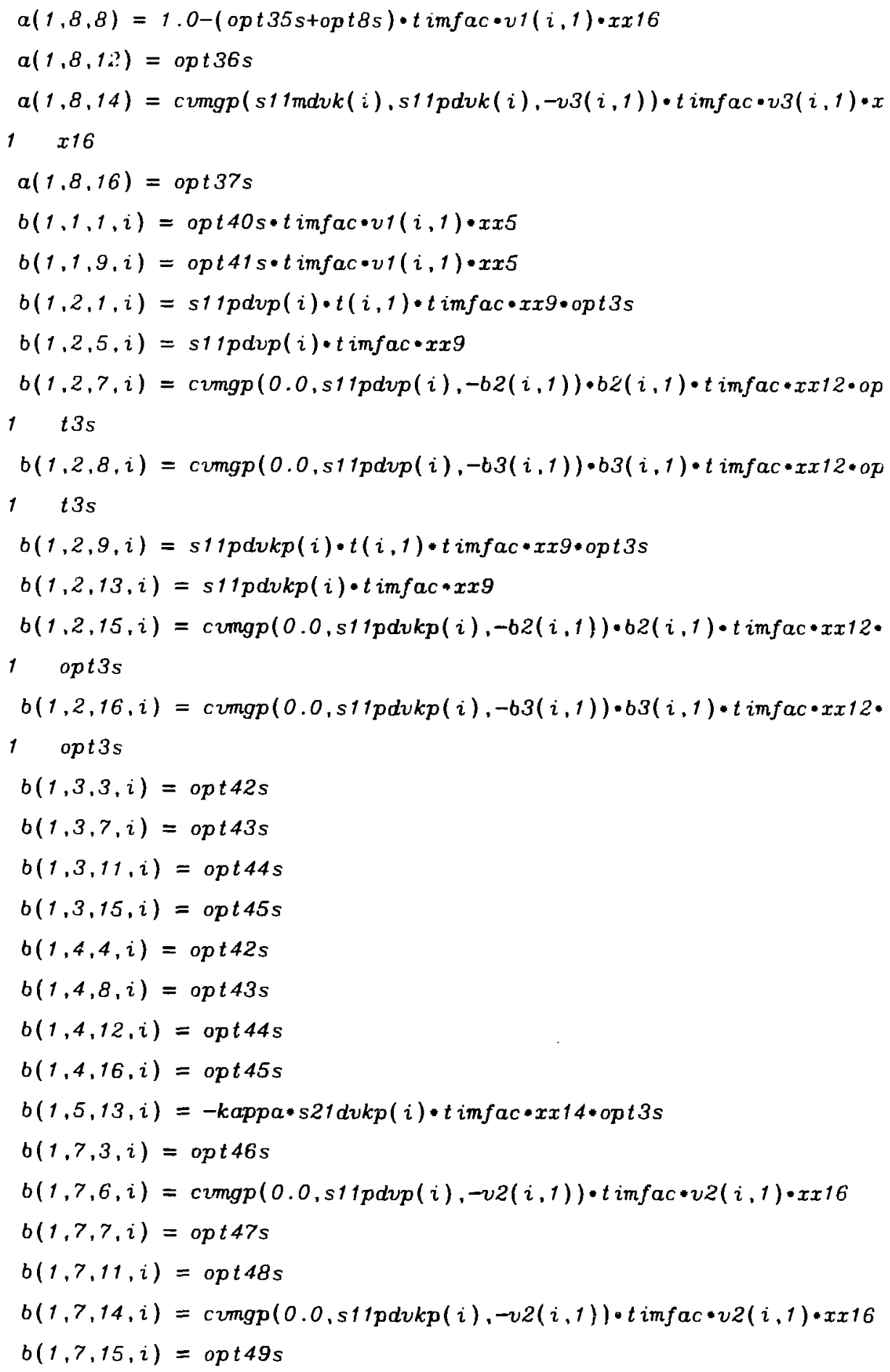




$$
\begin{aligned}
& b(1,8,4, i)=\text { opt } 46 \mathrm{~s} \\
& b(1,8,6, i)=\operatorname{cvmgp}(0.0, \operatorname{s11pdvp}(i),-v 3(i, 1)) \cdot \operatorname{timfac} \cdot v 3(i, 1) \cdot x x 16 \\
& b(1,8,8, i)=\text { opt } 47 \mathrm{~s} \\
& b(1,8,12, i)=\text { opt } 48 \mathrm{~s} \\
& b(1,8,14, i)=\operatorname{cvmgp}(0,0, \operatorname{sipdvkp}(i),-v 3(i, 1)) \cdot t i m f a c \cdot v 3(i, 1) \cdot x x 16 \\
& b(i, 8,16, i)=\text { opt } 49 \mathrm{~s} \\
& \operatorname{rhs}(1,1, i)=-t i m f a c \cdot(p r h o(i, 1) \cdot v 3 d z p(1)+\operatorname{rodzp}(1) \cdot v 3(i, 1)+o p t 15 s \cdot v \\
& 1 \quad 1(i, 1))+x x 5-r h o(i, 1)-h i s t r h o(i, 1) \\
& \operatorname{rhs}(1,2, i)=-t i m f a c *((t \operatorname{trp}(1)+\operatorname{rodrp}(1) \cdot t(i, 1) * \operatorname{opt} 3 s) \cdot x x 9-\operatorname{opt} 17 s * 0 \\
& 1 p t 19 s+(v 1 d z p(1) \cdot v 3(i, 1) \cdot x x 8+(b 3(i, 1) \cdot(\text { optz1s-b1dzp }(1))+b 2(i, 1) * \\
& 2 \text { opt20s) } x x 12) \cdot o p t 3 s)-v 1(i, 1)-h i s t v 1(i, 1) \\
& \operatorname{rhs}(1,3, i)=-t \text { imfac*(opt22s*opt19s+((v2dzp }(1) * v 3(i, 1)+v 1(i, 1) \cdot o p t \\
& 123 s) * x x 8-(b 2 d z p(1) * b 3(i, 1)+b 1(i, 1) \cdot o p t 24 s) \cdot x x 12) \cdot o p t 3 s)-v 2(i, 1) \\
& 2 \quad-h i s \operatorname{tv2}(i, 1) \\
& \operatorname{rhs}(1,4, i)=-t i m f a c \cdot(t d z p(1) \cdot x x 9+(\operatorname{rodzp}(1) \cdot t(i, 1) \cdot x x 9+(v 3(i, 1) \cdot v 3 \\
& 1 d z p(1)+v 1(i, 1) \cdot o p t 28 s) \cdot x x 8+(-b 1(i, 1) \cdot c u m g p(b 3 d r m(1), b 3 d r p(1), b 1 \\
& 2(i, 1))+b 2(i, 1) \cdot b 2 d z p(1)+b 1(i, 1) \cdot b 1 d z p(1)) \cdot x x 12) \cdot o p t 3 s)-v 3(i, 1)- \\
& 3 \text { instv3(i,1) } \\
& \operatorname{rhs}(1,5, i)=-t i m f a c \cdot(t(i, 1) \cdot(v 3 d z p(t)+v 1(i, 1) \cdot \text { opt } 8 s) \cdot x x i 5-k a p p a \cdot( \\
& 1 \quad t d r m(1) \cdot o p t 8 s+t d 2 z p(i)+t d 2 r(1)) \cdot x x 14 \cdot o p t 3 s)-t(i, 1)-h i s t t(i, 1) \\
& \operatorname{rhs}(1,6, i)=\operatorname{timfac} \cdot(b 1(i, 1) \cdot v 3 d z p(1)+b 1 d z p(1) \cdot v 3(i, 1)-(b 3(i, 1) \cdot v 1 \\
& 1 \quad d z p(1)+b 3 d z p(1) \cdot v 1(i, 1))) \cdot x x 16-h i s t b 1(i, 1)-b 1(i, 1) \\
& \operatorname{rhs}(1,7, i)=\operatorname{timfac} \cdot(b 2(i, 1) \cdot v 3 d z p(1)+b 2 d z p(1) \cdot v 3(i, 1)-(b 3(i, 1) \cdot v 2 \\
& 1 d z p(1)+b 1(i, 1) * o p t 34 s+(b 3 d z p(1)+o p t 32 s) * v 2(i, 1))+c u m g p(b 2 d r m(1) \\
& 2 \quad, b 2 \operatorname{drp}(1), v 1(i, 1)) \cdot v 1(i, 1)) \cdot x x 16-h i s t b 2(i, 1)-b 2(i, 1) \\
& \operatorname{rhs}(1,8, i)=-t i m f a c \cdot(b 1(i, 1) \cdot o p t 39 s+(b 1(i, 1) \cdot v 3(i, 1)-b 3(i, 1) \cdot v 1(i \\
& 1,1)) * o p t 8 s+o p t 38 s * v 3(i, 1)+c v m g p(-b 3 \operatorname{drm}(1),-b 3 \operatorname{drp}(1), v 1(i, 1)) \cdot v 1 \\
& 2(i, 1)) \cdot x x 16-h i s t b 3(i, 1)-b 3(i, 1)
\end{aligned}
$$

Finally, there remains the declarations section. It is contained in the file "gcookiglobal declrs": 
common /geomr/s11mdv(n)

common /difrho/ $\operatorname{rodrmi}(l d), \operatorname{rodrp}(l d), \operatorname{rod} 2 r(l d), \operatorname{rodzm}(l d), \operatorname{rodzp}(l d)$,

$1 \operatorname{rod} 2 z(l d), \operatorname{rod} 2 r m(m), \operatorname{rod} 2 r p(m), \operatorname{rod} 2 z m(n), \operatorname{rod} 2 z p(n)$

common /difv1/ v1drm(ld),v1drp(ld),v1d2r(ld),v1dzm(ld),v1dzp(ld),v

$1 \quad 1 d 2 z(l d), v 1 d 2 r m(m), v 1 d 2 r p(m), v 1 d 2 z m(n), v 1 d 2 z p(n)$

common /difvz/ v2drm( $l d), v 2 d r p(l d), v 2 d 2 r(l d), v 2 d z m(l d), v 2 d z p(l d), v$

I $\quad 2 d 2 z(l d), v 2 d 2 r m(m), v 2 d 2 r p(m), v 2 d 2 z m(n), v 2 d 2 z p(n)$

comonon /difv3/ v3drm(ld),v3drp $(l d), v 3 d 2 r(l d), v 3 d z m(l d), v 3 d z p(l d), v$

$1 \quad 3 d 2 z(l d), v 3 d 2 r m(m), v 3 d 2 r p(m), v 3 d 2 z m(n), v 3 d 2 z p(n)$

comonon /dift/ $t d r m(l d), t d r p(l d), t d 2 r(l d), t d z m(l d), t d z p(l d), t d 2 z(l d$

$1 \quad), t \alpha 2 r m(m), t d 2 r p(m), t d 2 z m(n), t d 2 z p(n)$

common /difbl/ b1drm(ld),b1drp(ld),b1d2r(ld),b1dzm(ld),b1dzp(ld),b

$1 \quad 1 d 2 z(l d), b 1 d 2 r m(m), b 1 d 2 r p(m), b 1 d 2 z m(n), b 1 d 2 z p(n)$

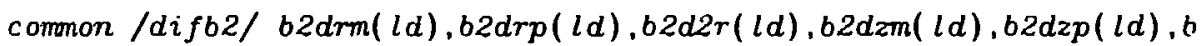

$12 d 2 z(l d), b 2 d 2 r m(m), b 2 \alpha 2 r p(m), b 2 d 2 z m(n), b 2 d 2 z p(n)$

common /difb3/ b3drm(ld),b3drp $(l d), b 3 d 2 r(l d), b 3 d z m(l d), b 3 d z p(l d), b$

$f \quad 3 d 2 z(l d), b 3 d 2 r m(m), b 3 d 2 r p(m), b 3 d 2 z m(n), b 3 d 2 z p(n)$

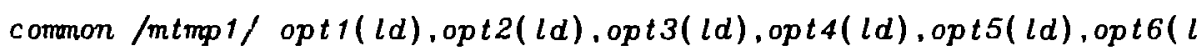

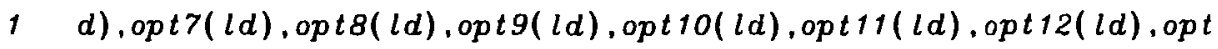

$2 \quad 13(l d)$.opt14(ld),opt15(ld),opt16(ld),opt17(ld),opt18(ld),opt19(

$3(l d), o p t 20(l d)$, opt21(ld),opt22(ld),opt23(ld),opt24(ld).opt25(ld)

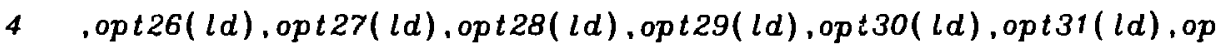

$5 \quad t 32(l d)$, op $t 33(l d)$, opt $34(l d)$, opt $35(l d)$, opt $36(l d)$, op $t 37(l d)$, op $t 38$

$6 \quad(l d)$, opt $39(l d)$, opt $40(l d)$

common /mtmp2/ opt41(ld), opt42(ld), opt43(ld), opt44(ld), opt45(ld), o

$1 p t 46(l d)$, opt $47(l d)$, opt $48(l d)$, opt $49(l d)$, opt5o( $l d)$, opt51(ld), opt5

$22(l d)$, opt $53(l d)$, opt54(ld),opt55(ld),opt56(ld),opt57(ld),opt58( $l$

$3 d), o p t 59(l d), o p t 60(l d), o p t 61(l d), o p t 62(l d), o p t 63(l d)$, opt64( $(d)$.

4 opt65(ld),opt66(ld),opt67(ld).opt68(ld).opt69(ld),opt70(ld),opt

$57 f(l d)$, opt $72(l d)$

common /cnst1/ picon,c,scltim,scalt,sclrho, scaleb, scalev,gam, csubv

1 , coeu1k, coeu2k, coeu3k, coek2k, coek3k, coeunk, coeunmk, coeunm2k, coe

$2 k$ knk, coeknm2k, coeu1 $l, \operatorname{coeu} 2 l, \operatorname{coeu} 3 l, \operatorname{coe} l 2 l$, coe $l 3 l$, coeun $l, \operatorname{coeumm} l$

3 , coeumm2l, coelnml, coelnm2 l, xx1, xx2, xx3, xx4, xx5, xx6, xx7, xx8, xx9, 
$x x 10, x x 11, x x 12, x x 13, x x 14, x x 15, x x 16, x x 17$, opt $1 \mathrm{~s}$, opt $2 \mathrm{~s}$, opt $3 \mathrm{~s}$, opt $4 \mathrm{~s}$ ,opt5s, opt6s, opt7s, opt8s, opt9s, opt10s, op!11s, opt12s, opt13s, opt1 4s, opt15s, opt16s, opt17s, opt18s, opt19s, opt20s, opt21s, opt22s, opt2 3s, opt24s, opt25s, opt26s, opt27s, opt28s, opt29s, opt30s, opt31s, opt3 $2 s$, opt33s, opt34s, opt35s, opt36s, opt37s, opt $38 \mathrm{~s}$, opt $39 \mathrm{~s}$, op $t 40 \mathrm{~s}$, opt 4

1s, opt 42s, opt43s, opt44s, opt 45s, opt46s, opt47s, opt 48s, opt49s

data picon,, sc $l t i m$, scalt/3.14159265.2.9979e+10,1.0e-6,1.602e-9/ data sclrho, scaleb, scalev,gom/1.0,1000000.0,1000000.0,1.66666667/ data csubv/2.1/ pointer (mrhs, rhs $(l d, n e q, l d)),($ ma, $a(l d, n e q, n e q))$ pointer $(m b, b(l d, n e q, n e q, l d)),(m r h o, r h o(n, m)),(m r h o k, r h o k(n, m)),(m$ 1 rhol, rhol $(n, m)),($ mrhopas, rhopas $(r, m)),(\operatorname{mrhopas} 2, \operatorname{rhopas} 2(n, m)),($ mrhokpas, rhokpas $(n, m)),($ mrholpas, rholpas $(n, m)),(m v 1, v 1(n, m)),(m$

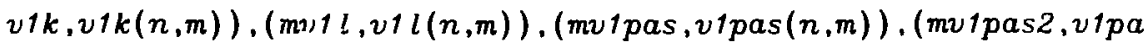
$s 2(n, m)),(\operatorname{mu1kpas,v1kpas}(n, m)),(\operatorname{mv1} l p a s, v 1 \operatorname{lpas}(n, m)),(m v 2, v 2(n$,

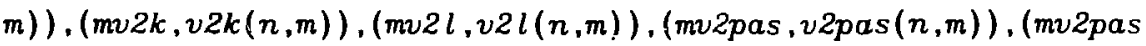

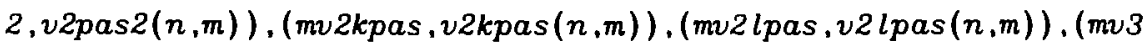
$, v 3(n, m)),(m v 3 k, v 3 k(n, m)),(m v 3 l, v 3 l(n, m)),(m v 3 p a s, v 3 p a s(n, m)),($ mv3pas2, v3pas2(n,m)),(mv3kpas,v3kpas $(n, m)),($ mv3lpas , $23 \operatorname{lpas}(n, m)$ )$,(m t, t(n, m)),(m t k, t k(n, m)),(m t l, t i(n, m)),(m t p a s, t p a s(n, m)),(m t$ pas $2, t \operatorname{pas} 2(n, m)),(m t k p a s, t k p a s(n, m)),(m t l p a s, t l p a s(n, m)),(m b 1, b$ ; $\quad l(n, m)),(m b 1 k, b 1 k(n, m)),(m b 1 l, b 1 l(n, m)),(m b 1 p a s, b 1 p a s(n, m)),(m b$ < $\quad 1 p a s 2, b 1 p a s 2(n, m)),(m b 1 k p a s, b 1 k p a s(n, m)),(\operatorname{mb} 1 \operatorname{lpas}, b 1 l p a s(n, m))$,

$=(m b 2, b 2(n, m)),(m b 2 k, b 2 k(n, m)),(m b 2 l, b 2 l(n, m)),(m b 2 p a s, b 2 p a s(n, m$

- $\quad),($ mb2pas 2, b2pas $2(n, m)),(\operatorname{mb2kpas,b2kpas}(n, m)),(m b 2 l p a s, b 2 l p a s($ $n, m)),(m b 3, b 3(n, m)),(m b 3 k, b 3 k(n, m)),(m b 3 l, b 3 l(n, m)),(m b 3 p a s, b 3 p$ $a s(n, m)),(m b 3 p a s 2, b 3 p a s 2(n, m)),(m b 3 k p a s, b 3 k p a s(n, m)),(m b 3 l p a s, b$

$2 \quad 3 l p a s(n, m))$

pointer (mc,c(ld,neq,neq))

common buffer (40)

if (minit(109,buffer).ne.0) go to 9999

if (malloc("rhs",mrhs,ld*ld*neq).ne.0) go to 9999

if (malloc("a",ma, ld*neq*neq).ne.0) go to 9999

if $($ malloc("b",mb,ld•neq*neq•ld).ne.0) go to 9999 
if (malloc("c",mc,ld•neq•neq).ne.o) go to 9999

if (malloc("rho",mrho,m*n).ne.0) go to 9999

if (malloc("rhok",mrhok,m*n).ne.0) go to 9999

if (malloc("rhol",mrhol,m*n).ne.o) go to 9999

if (malloc("rhopas",mrhopas,m*n).ne.0) go to 9999

if (malloc("rhopas2".mrhopas2,m・n).ne.0) go to 9999

if (malloc("rhokpas",mrhokpas,m*n).ne.0) go to 9999

if (malloc("rholpas".mrholpas,m*n).ne.0) go to 9999

if $($ malloc("v1",mv1,m•n).ne.0) go to 9999

if (malloc("v1k",mv1k,m॰n).ne.0) go to 9999

if(malloc("v1l",mv1l,m*n).ne.0) go to 9999

if (malloc("v1pas",mu1pas,m॰n).ne.0) go to 9999

if (malloc("v1pas2",mv1pas2,m*n).ne.0) go to 9999

if (malloc("v1kpas",mv1kpas,m•n).ne.0) go to 9999

if (malloc("v1 lpas",mul lpas,m・n).ne.0) go to 9999

if (malloc("v2",mv2,m・n).ne.0) go to 9999

if (mal loc("v2k",mv2k,m*n).ne.0) go to 9999

if (malloc(" $v 2 l ", m v 2 l, m \cdot n) . n e .0)$ go to 9999

if(malloc("v2pas",mv2pas,m・n).ne.0) go to 9999

if (malloc("v2pas2" ,mv2pas2,m*n) ne.0) go to 9999

if (malloc("v2kpas",mv2kpas,m•n).ne.0) go to 9999

if (malloc("v2lpas",mv2 lpas,m・n).ne.0) go to 9999

if (malloc("v3",mv3,m・n).ne.0) go to 9999

if (malloc("v3k",mv3k,m・n).ne.0) go to 9999

if (malloc("v3l",mv3l,m*n).ne.0) go to 9999

if (malloc("v3pas",mv3pas,m॰n) .ne.0) go to 9999

if (malloc("v3pas2",mv3pas2,m*n).ne.0) go to 9999

if (malloc("v3kpas",mv3kpas,m•n).ne.o) go to 9999

if (malloc("v3lpas",mu3lpas,man).ne.o) go to 9999

if (malloc("t",mt,m*n).ne.0) go to 9999

if (malloc("tk",mtk,m•n).ne.o) go to 9999

if (malloc(" $t l ", m t l, m * n) . n e .0)$ go to 9999

ij(malloc("tpas",mtpas,m・n).ne.0) go to 9999

if (malloc("tpas2",mtpas2,m•n).ne.0) go to 9999 
if (malloc("tkpas",mtkpas,m*n).ne.0) go to 9999

if (malloc("t lpas",mt lpas,m・n).ne.0) go to 9999

if (malloc("b1",mb1,m・n).ne.0) go to 9999

$i_{f}(\operatorname{malloc}(" b 1 k ", m b 1 k, m * n)$.ne.0) go to 9999

if (malloc("b1l",mb1l,m॰n).ne.0) go to 9999

if(malloc("b1pas",mb1pas,m*n).ne.0) go to 9999

if (malloc("b1pas2",mb1pas2,m・n).ne.o) go to 9999

if(malloc("b1kpas",mb1kpas,m*n).ne.0) go to 9999

if(malloc("b1 lpas",mb1 lpas,m*n).ne.0) go to 9999

if (malloc("b2",mb2,m*n).ne.0) go to 9999

if (malloc("b2k",mb2k,m*n).ne.0) go to 9999

if (malloc("b2l",mb2l,m*n).ne.o) go to 9999

if (mal Loc("b2pas",mb2pas,m•n) .ne.0) go to 9999

if (malloc("b2pas2",mb2pas2,m•n).ne.0) go to 9999

if (malloc("b2kpas",mb2kpas, m*n).ne.0) go to 9999

if (malloc("b2lpas",mb2lpas,m•n) .ne.o) go to 9999

if (malloc("b3",mb3,m・n).ne.0) go to 9999

if (malloc("b3k",mb3k,m・n).ne.0) go to 9999

if(malloc("b3l",mb3l,m*n).ne.0) go to 9999

if (malloc("b3pas",mb3pas,m•n).ne.0) go to 9999

if (malloc("b3pas2",mb3pas2,m•n).ne.0) go to 9999

if (malloc("b3kpas",mb3kpas,m•n).ne.o) go to 9999

if (malloc("b3lpas",mb3lpas,m•n).ne.o) go to 9999

$x x 1=g a m-1.0$

$x x 2=c \operatorname{subv} \cdot x x 1$

$x \times 3=1 / p i c o n$

$x x 4=c 1 d 4 \cdot c \cdot x x 3$

$x x 5=$ scalevesclrho

$x x 6=$ scalev $* 2$

$x x 7=1 /$ sclrho

$x x 8=x x 6 \cdot x x 7$

$x x 9=\operatorname{scalt} \cdot x x^{2}$

$x \times 10=1 / c$

$x x_{11}=\operatorname{scaleb} \cdot \cdot 2$ 


$$
\begin{aligned}
& x x 12=x x 10 \cdot x x 11 \cdot x x 4 \cdot x x 7 \\
& x x 13=1 / \text { csubv } \\
& x x 14=\text { scalt } x x 13 \cdot x x 7 \\
& x x 15=\text { scalev } x x 13 \cdot x x 9 \\
& x x 16=\text { scalebtscalev } \\
& x \times 17=-2.0 \cdot x x 8
\end{aligned}
$$

A comment in Chapter 6 should be reemphasized at this point. The above code segments are oniy a part of what would actually be generated to solve a two-dimensional version of the equations in Example 2. Moreover, there are other interface code segments and drivers required to make a complete code. Chapter 7 discusses some of these requirements. 


\section{ACKNOWLEDGMENTS}

I would like to thank many people for suggestions, discussions, and the ir help throughout the course of this work.

First, I wish to thank Larry Knight, who, as my advisor, encouraged me and persisted in not letting this work languish. His critical comments have been most helpful. Also, Helaman Ferguson and Mark Nelson are to be thanked for the ir support.

For their financial assistance during three years of this work, l am grateful to the Associated Western Universities for their fellowship; the BYU Physics department must also be thanked for their financial aid. Also in this connection, and for his hospitality in allowing me to use the facilities of the National Magnetic Fusion Energy Computer Center (NMFECC), l am very grateful to John Killeen. The staff at the NMFECC were most solitious of my needs. F'or example, the large MACSYMA computation could not have been done without the generous funding of the center. 
With regard to this document, I wish to thank Gary Kerbel for helping with the equations, Hank Moll for helping with RED macros, and Dale Nielsen, Jr. for introducing the whole novel idea to me.

For many invaluable discussions on computational physics, I wish to thank Irv Lindemuth, Dalton Schnack, Charlie Finan, Jim LeBlanc, and many others at Lawrence Livermore National Laboratory. Also, discussions on the upwinding issue with B. P. Leonard were greatly appreciated.

In the area of ordinary differential equation theory, I wish to thank Alan Hindmarsh for much valuable guidance, including making available to me some of his personal notes. Fred Fritsch is to be thanked for providing some important information on higher-order splines. Also, Philip Rabinowitz helped with evaluation of singular integrals.

For substantial LISP support programing and debugging help as well as MACSYMA suggestions, I am indebted to George Carrette. And for helping me through the complex conversion of the large MACSYMA run to the MULTICS computer, Jim O'Dell must be thanked. To others in the MACSYMA community who gave assistance, the Mathlab group in particular, I also of fer my thanks. Much of this dissertation was done with the aid of MACSYMA, a large symbolic manipulation program developed at the MIT Laboratory for Computer Science and supported by the National Aeronautics and Space Administration under grant NSG 1323, by the Office of Naval Research under grant N00014-77-C-0641, by the $U$. 
S. Department of Energy under grant ET-78-C-02-4687, and by the U. S. Air Force under grant F49620-79-C-020.

During the several years when this work was going on, my family has never waivered in their support. Without this support, it could not have been done. In addition, I wish to thank my mother-in-law for reading the manuscript and suggesting valuable improvements. Most especially, I wish to thank my wife for her extra sacrifices in typing for me and sustaining me through these trying years. 


\section{BIBLIOGRAPHY}

(1) Milton Abramowitz and Irene A. Stegun. Handbook of Mathemalical Functions

(U.S. Government Printing of fice, Washington D.C., 1972) pp. 576, 598, 608-609.

(2) David V. Anderson and Daniel C. Barnes, Accurate Calculations of Field-Reversed Axisymetric Equilibria and Their MHD Stability Properties, $J$. Comput. Phys. 42, 288-308 (1981).

(3) R. Balescu and J. H. Misguich, Kinetic equations for plasmas subjected to a strong time-dependent external field, Part 1. General theory, J. Plasma Phys. 11, 357-375 (1974), Part 2. The weak-interaction approximation, $J$. Plasma Phys. 11, 377-387 (1974).

(4) Richard M. Beam and R. F. Warming, An Implicit Factored Scheme for the Compressible Navier-Stokes Equations II: The Numerical ODE Connection, in Proc. AlAA 4th Computational Fluid Dymanics Conference, Williamsburg, Va., pp. 1-13 (1979)

(5) Richard. M. Beam and R. F. Warming, Alternating Direction Implicit 
Me'hods for Parabolic Equations with a Mixed Derivative, SIAM J. Sci. Stat. Compret. 1. 131-159 (1980).

(6) G. H. Behforooz and N. Papmichael, End Conditions for Cubic Spline Interpolation, $J$. Inst. Maths. ${ }_{4}^{4 p p l i c s .23,355-366 ~(1979) . ~}$

(7) Carl A. de Boor, Practical Guide to Splines, (Springer-Verlag, New York. 1978), pp. 129-153, 227-298.

(8) J. U. Brackbill and D. C. Barnes. The effect of Nonzero $\nabla \cdot B$ on the Numerical Solution of the Magnetohydrodynamic Equations, J. Comput. Phys. 35, $426-430(1980)$.

(9) S. I. Braginski i, Transport Processes in a Plasma, in Reviews of Plasma Physics, edited by M. A. Leontovich (Consultants Bureau, New York, 1965), Vol. 1, pp. 205-311.

(10) W. R. Briley and H. McDonald, Solution of the Multidimensional Compressible Navier-Stokes Equations by a Generalized Implicit Method, $J$. Comput. Phys. 24, 372-397 (1977).

(11) W. R. Briley and H. McDonald, On the Structure and Use of Linearized Block Implicit Schemes, J. Comput. Phys. 34, 54-73 (1980).

(12) Hermann Brunner and Syvert P. Norsett, Superconvergence of Collocation 
Methods for Volterra and Abel Integral Equations of the Second Kind, Nomer. Math. 36. 347-358 (1981).

(13) Ingrid Yvonne Bucher. An Abstract Model of the Finite Element Method. M.S. Thesis, Los Alamos Scientific Laboratory, Los Alamos, New Mexico, LA-8468-T $(1980)$.

(14) R. Bulirsch. Numerical Calculation of Elliptic Integrals and Elliptic Functions. III, Namer. Math. 13, 305-315 (1969).

(15) Paul F. Byrd and Morris D. Friedman, Handbook of Elliptic Integrals for Engineers and Physicists (Springer-Verlag. Berlin, 1954) pp. 176, 282.

(16) S. Cabay and T. P. L. Lam, Algor ithm 522. ESOLVE, Congruence Techniques for the Exact Solution of Integer Systems of Linear Equations, ACM Trans. Math. Soft. 3, 404-410 (1977).

(17) R. E. Carlson and F. N. Fritsch, Monotone Piecewise Bicubic Interpolation, Lawrence Livermore National Laboratory, Livermore, Calif., UCRL $-86449(1981)$.

(18) W. P. Chandler, Coupled MHD-Monte Carlo Transport Model for Dense Plasmas, Ph.D. Thesis, Lawrence Livermore Laboratory, Livermore, Calif.. UCRL-52009 (1975). 
(19) Shui-Nee Chow, John Mallet-Paret and James A. Yorke, Finding Zeroes of Maps: Homotopy Methods that are Constructive with Probability One, Math. Comp. 32. Bธ7-899 (1978).

(20) M. S. Chu, Hot plasma in contact with a cold wall, Phys. Fluids i6, $1441-1445$ (1973)

(21) W. J. Cody. Chebyshev Approximations for the Complete Elliptic Integrals $K$ and $E$, Math. Comp. 19, 105-112 (1965).

(22) W. J. Cody, Chebyshev Polynomial Expansions of Complete Elliptic Integrals, Math. Comp. 19, 249-259 (1965).

(23) Bruce I. Cohen, Robert P. Freis, and Vincent Thomas, Orbit-Averaged Implicit Particle Codes, J. Comput. Phys. 45, 345-366 (1982).

(24) Grant Cook, Jr. and Larry Knight, On Optimal Reconstruction Angles, in Proc. Sixth Conference on Computer Applications in Radiology and Computer/Aided Analysis of Radiological Images, 291-304 (1979).

(25) G. Dahlquist, A special stability problem for linear multistep methods, $B I T 3,27-43(1963)$.

(26) Germund Dahlquist, Some Contractivity Questions for One-Leg and Linear 
Multistep Methods, Dept. Comp. Sci., Royal Institute of Technology, Stockholm, Report TRITA-NA-7905 (1979).

(27) R. C. Davidson and N. A. Krall, Anomalous Transport in High-Temperature Plasmas with Applications to Solenoidal Fusion Systems, Nuclear Fusion 17. $1313-1372(1977)$.

(28) Huw C. Davies, A Pseuro-Upstream Differencing Scheme for Advection, $j$. Comput. Phys. 37, 280-286 (1980).

(29) Harold T. Davis, Introduction to Nonlinear Differential and Integral Equations (Dover Publications, New York, 1962), pp. 170-171.

(30) Philip J. Davis and Philip Rabinowitz. Methods of Numerical Integration (Academic Press, New York, 1975), pp. 114-116, 268-273, 369.

(31) Elise de Doncker and Robert Piessens, Automatic Computation of Integrals with Singular Integrand, Over a Finite or an Infinite Range. Applied Math and Programming Division, Kotholieke Universiteit Leuven, Report TW 22 (1975).

(32) H. W. Drawin and F. Emard, Instantaneous Population Densities of the Excited Levels of Hydrogen Atoms and Hydrogen-Like lons in Plasmas, Physica 85c. $333-356(1977)$.

(33) Dwight Duston and James J. Duderstadt, Ionization and radiation dynamics 
of dense MHD plasmas, J. Appl. Phys. 49. 4388-4395 (1978).

(34) Bjorn Enquist and Tom Sinedsaas, Automatic Computer Code Generation for Hyperbolic and Parabolic Differential Equations, SIAM J. Sci. Stat. Comput. 1. 249-259 (1980).

(35) H. Fasel, Recent Developments in the Numerical Solution of the Navier-Stokes Equations and Hydrodynamic Stability Problems, in Computational Fluid Dynamics, edited by Wolfgang Kollman, (Hemisphere Publishing Corporation, Washington, 1980), pp. 167-279.

(36) Helaman Rolfe Pratt Ferguson, Dale E. Nielsen and Grant Cook, A Partition Formula for the Integer Coefficients for the Theta Function Nome, Nath. Comp. 29, 851-855 (1975).

(37) Helaman Rolfe Pratt Ferguson, private communication.

(38) Charles H. Finan III, The alternating-direction implicit numerical solution of the time-dependent, three-dimensional, single fluid, resistive magnetohydrodymamic equations, Ph.D. Thesis, Lawrence Livermore National Laboratory, Livermore, Cal if .,UCRL-53086 (1980).

(39) Joseph E. Flaherty and William Mathon, Collocation with Polynomial and Tension Splines for Singularly-Perturbed Boundary Value Problems, SIAM J. Sci. Stat. Comput. 1,260-289 (1980). 
(40) Walter Gautschi, Questions of Numerical Condition Related to Polynomials. in Recent Advances in Nomerical Analysis, edited by Carl de Boor and Gene $H$. Golub (Academic Press. New York, 1978), pp. 45-72.

(41) R. C. Goldfinger, Formulas and Subroutines for $\boldsymbol{\theta}, \boldsymbol{\nabla e}$, and $\psi$ of a Current Loop with Application to Magnetic Fusion Experiments, Oak Ridge National Laboratory, Oak Ridge, Tennessee, ORNL/TM-7636 (1981).

(42) Philip M. Gresho, and Robert L. Lee, Don't Suppress the Wiggles - They're Telling You Something. Computers and Fluids 9, 223-253 (1981).

(43) J. Hackl, Hj. Wacker, and W. Zulehner, An Efficient Step Size Control for Continuation Methods, BIT 20,475-485 (1980).

(44) R. W. Hamming, Namerical Methods for Scientists and Engineers (McGraw-Hill, New York, 1973), p. 235.

(45) G. W. Hedstrom and G. H. Fodrigue, Adaptive-grid methods for time-dependent partial differentiai equations, Lawrence Livermore National Laboratory, Livermore, Cal if ., UCRL-87242 (1982).

(46) A. C. Hindmarsh, Solution of Block-Tridiagonal Systems of Linear Algebraic Equations, Lawrence Livermore Laboratory, Livermore, Calif.. UCID-30150 (1977). 
(47) Morris W. Hirsch and Stephen Smale, On Algorithms for Solving $f(x)=0$. Comun. Pure Appl. Math. 32, 281-312 (1979).

(48) M. A. Hogge, A Comparison of Two- and Three-Level Integration Schemes for Non-Linear Heat Conduction, in Nomerical Methods in Heat Transfer, edited by R. W. Lewis, K. Morgan, and 0. C. Zienkiewicz (Wiley, New York, 1981), pp. 75-90.

(49) Elias Houstis, A Collocation Method for Systems of Nonlinear Ordinary Differential Equations, J. Math. Anal. Appl. 62, 24-37 (1978).

(50) E. N. Houstis, R. E. Lynch and J. R. Rice, Evaluation of Numerical Methods for Elliptic Partial Differential Equations, J. Comput. Phys. 27, 323-350 (1978)

(51) K. P. Huber and G. Herzberg, Molecular Spectra and Molecular Structure, IV. Constants of Diatomic Molecules (Van Nostrand Reinhold Co., New York. 1979), pp. 262-267.

(52) John David Jackson, Classical Electrodymanics (Wiley, New York, 1962), pp. $141-142,199$

(53) Jahnke-Emde-Losch, Tables of Higher Functions (B. G. Teubner, Stutlgart, 1960), pp. 62, 82-89. 
(54) B. K. Jensen, Physics of a fusion plasma boundary layer, Phys. Fluids $20,373-378(1977)$.

(55) Edward J. Kansa. The Disparate Spatial and Temporal Scale Problem in Combustion Modeling: A Proposed Remedy, Lawrence Livermore National Laboratory, Livermore, Calif ., UCID-19248 (1981).

(56) Herbert B. Keller, Global Homotopies and Newton Methods, in Recent Advances in Nomerical Analysis, edited by Carl de Boor and Gene H. Golub (Academic Press, New York, 1978), pp. 73-94.

(57) David S. Kershaw, The Solution of Single Linear Tridiagonal Systems and Vectorization of the ICCG Algorithm on the CRAY 1, Lawrence Livermore Laboratory, Livermore, Calif .,UCID-19085 (1981).

(58) P. K. Khosla and S. G. Rubin, Filtering of Non-linear Instabilities, $J$. Eng. Math. 13, 127-141 (1979).

(59) J. Killeen, Difference Methods in Fluid Dynamics with Applications, in Computing as a Longuage of Physics (International Atomic Energy Agency, Vienna, 1972). pp. 129-156.

(60) J. Killeen, Computer Models of Magnetically Confined Plasmas, Nuclear Fusion 16, 841-864 (1976). 
(61) John Killeen, Computational Problems in Magnetic Fusion Research, Lawrence Livermore Laboratory, Livermore, Cal if ., UCRL-85440 (1981).

(62) Emil J. Konopinski, Electromagnetic Fields and Relativistic Particles (McGraw-Hi 11, New York, 1981), pp. 592-599.

(63) Nicholas A. Krall and Alvin W. Trivelpiece, Principles of Plasma Physics (McGraw-Hill. New York, 1973), pp. 82-97.

(64) E. W. Laing, Transport Theory, in Plasma Physics and Nuclear Fusion Research, edited by Richard D. Gill (Academic Press, London, 1981), pp. 155-189.

(65) J. D. Lambert, Stiffness, in Computational Techniques for Ordinary Differential Equations, edited by I. Gladwell and D. K. Sayers (Academic Press, London, 1980).

(66) B. P. Leonard, A Consistency Check for Estimating Truncation Error Due to Upstream Differencing, Appl. Math. Modelling 2, 239-244 (1980).

(67) B. P. Leonard, A Stable and Accurate Convective Modelling Procedure Based Quadratic Upstream Interpolation, Computer Methods in Applied Mechanics and Engineering 19, 59-98 (1979).

(68) B. P. Leonard, A Survey of Finite Differences of Opinion on Numerical 
Muddling of the Incomprehensible Defective Confusion Equation, in Finite Element Methods for Convection Dominated Flows, edited by T. J. R. Hughes (American Society of Mechanical Engineers, New York, 1980), pp. 1-17.

(69) Irvin R. Lindemuth, The Alternating-Direction Implicit Namerical Solution of Time-Dependent, Two-Dimensional, Two-Fluid Magnetohydrodymamic Equations, Ph.D. Thesis, Lawrence Livermore Laboratory, Livermore, Calif ., UCRL-51103 (1971).

(70) Irvin R. Lindemuth, Conservation Form in Computational Magnetohydrodynamics, $J$. Comput. Phys. 18, 119-131 (1975).

(71) Irvin R. Lindemuth, A Boundary Condition for Computational Magnet ohydrodynamics, J. Comput. Phys. 25, 104-117 (1977).

(72) I. R. Lindemuth, The Animal Code, Lawrence Livermore Laboratory. Livermore, Calif., UCRL-52492 (1979).

(73) Irvin R. Lindemuth and John Killeen, Alternating Direction Implicit Techniques for Two-Dimensional Magnetohydrodynamic Calculations, $J$. Comput. Phys. 13, 181-208 (1973).

(74) I. R. Lindemuth, J. S. Pettibone, J. C. Stevens, R. C. Harding, D. M. Kraybill, and J. L. Suter, Unstable Behavior of Hot, Magnetized Plasma in Contact with a Cold Wall, Phys. Fluids 21, 1723-1734 (1978). 
(75) I. R. Lindemuth, J. S. Pettibone, J. C. Stevens, D. M. Kraybill, J. L. Suter and R. C. Harding, The Numerical Computation of the Preionization Phase of a Toroidal Pinch Discharge, Plasma Physics 22, 207-225 (1980).

(76) John Locker and P. M. Prenter, Regularization with Differential Operators. I. General Theory, J. Math. Anal. Appl. 74, 504-529 (1980), II: Weak Least Squares Finite Element Solutions To First Kind Integral Equations, SIAM J. Numer. Anal. 17, 247-267 (1980).

(77) H. C. Lui and C. K. Chu, Two-dimensional magnetohydrodynamic simulation of toroidal pinches, Phys. Fluids 18, 1277-1281 (1975).

(78) Brendan McNamara, Computing in Magnetic Fusion, Proc. of the IEEE 69, 1043-1055 (1981).

(79) Donald A. McQuarrie, Statistical Mechanics (Harper and Row, New York, 1976), pp. 91-112.

(80) J. H. Mcthirter, R. J. Duffin, P. J. Brehm, and J. J. Oravec, Computational Methods for Solving Static Field and Eddy Current Problems via Fredholm Integral Equations, IEEE Trans. MAG-15, 1075-1084 (1979).

(81) MACSMA Reference Manual. Project MAC Mathlab Group, MIT (1978).

(82) D. F. Mayers, Quadrature Methods for Fredholm Equations of the Second 
Kınd, in Numerical Solution of Integral Equations, edited by L. M. Delves and J. Walsh (Clarendon Press, Oxford, 1974).

(83) M. D. Mikhailov and M. A. Aladjem, Automatic Solution of Thermal Problems, in Namerical Methods in Heat Transfer, edited by R. W. Lewis, K. Morgan, and 0. C. Zienkiewicz (Wiley, New York, 1981), pp. 27-49.

(84) Marvin E. Morris, Admittance of an Infinitely Long Monopole Antenna Immersed in a Dissipative Medium and Driven by an Air-Filled Coaxial Line. Sandia National Laboratories, Albuquerque, New Mexico. SAND79-1401 (1980).

(85) H. M. Nelson, R. W. Bass, L. Fearnley, H. R. P. Ferguson, J. H. Gardner, B. K. Harrison, K. M. Larsen, D. E. Nielson, G. Cook, L. Scott. R. Wilson, and W. Young, Physical Realizability of the Topolotron Configuration, Proc. Utah Acad. Sci.,Arts, Lett. 50,47-57 (1973).

(86) H. Mark Nelson, Keith H. Brown and Charles A. Hart, Computer Model of a Fast Toroidal Plasma Compression, with Application to the Topolotron. Phys. Fluids $19,1810-1819(1976)$.

(87) Olavi Nevanlinna and Werner Liniger, Contractive Methods for Stiff Differential Equations, Part I, $B I T$ 18, 457-474 (1978), Part II, $B I T 19,53-72$ $(1.979)$.

(88) Edward W. Ng and Bruce Char, Gradient and Jacobian Computation for 
Numerical Applications, in Proc, 1979 MACSYMA Users Conference, Washington, D. C., pp. 604-621 (1979).

(89) G. H. Nickel, Elementary Derivation of the Saha Equation, Am. J. Phys. $48,448-450(1980)$.

(90) Paul Douglas Nielsen, A Computational Investigation of the Limits to Pease-Braginskii Collapse of a Z-Pinch, Ph.D. Thesis, Lawrence Livermore National Laboratory, Livermore, Calif , UCRL-53166 (1981).

(91) D. E. Post, R. V. Jensen, C. B. Tarter, W. H. Grasberger, and W. A. Lokke, Steady-State Radiative Cooling Rates for Low-Density High-Temperature Plasmas, Atomic Data and Nuclear Data Tables 20, $397-439$ (1977).

(92) John R. Reitz and Frederick J. Milford, Foundations of Electromagnetic Theory (Addison-Wesley, Reading, MA, 1967), pp. 172-176.

(93) G. R. Richter, Numerical Solution of Integral Equations of the First Kind with Nonsmooth Kernels, SIAM J. Namer. Anal. 15, 511-522 (1978).

(94) Gerard R. Richter, Superconvergence of Piecewise Polynomial Galerkin Approximations, for Fredholm Integral Equations of the Second Kind, Numer. Math. 31, 63-70 (1978).

(95) R. D. Richtmyer and K. W. Morton, Difference Methods for Inital Value 
Problems (Wiley, New York, 1967).

(96) P. J. Roache, Computational Fluid Dymanics (Hermosa, Albuquerque, 1976).

(97) Keith V. Roberts and D. E. Potter. Magnetohydrodynamic Calculations, in Methods in Computational Physics, edited by Berni Alder, Sidney Fernbach, and Manuel Rotenberg (Academic Press, New York, 1970), Vol. 9, p. 339.

(98) F. J. Rogers, H. C. Graboske, Jr., and D. J. Harwood, Bound Eigenstates of the Static Screened Coulomb Potential, Phys. Rev. A 1. $1577-1586(1970)$.

(99) Stanley G. Rubin and Randolph A. Graves, Jr., A Cubic Spline Approximation for Problems in Fluid Mechanics, School of Engineering, Old Dominion University, Norfolk, Va., Report 74-T1 (1974).

(100) Stanley G. Rubin and Randolph A. Graves, Jr., Viscous Flow Solutions with a Cubic Spline Approximation. Computers and Fluids 3, 1-36 (1975).

(101) S. G. Rubin and P. K. Khosla, Higher-Order Numerical Methods Derived f. Jm Three-Point Polynomial Interpolation, National Aeronautics and Space Administration, Washington, D. C., CR-2735 (1976).

(102) S. G. Rubin and P. K. Khosla, Polynomial Interpolation Methods for Viscous Flow Calculations, J. Comput. Phys. 24, 217-244 (1977). 
(103) Steven J. Sackett, EFFI - A Code for Calculating the Electromagnetic Field, Force, and Inductance in Coil Systems of Arbitrary Geometry, Lawrence Livermore Laboratory, Livermore, Cal if ., UCRL-52402 (1978).

(104) Larry L. Schumaker, Spline Functions: Basic Theory. (Wiley, New York. 1981). pp. i39-209, 484-509.

(105) P. K. Sakanaka, Macroscopic Plasma Properties and Stability Theory, in Modern Plasma Physics, Proc. International Center for Theoretical Physics College, Trieste, 1979 (International Atomic Energy Agency, Vienna, 1981), pp. $3-45$.

(106) Dalton D. Schnack, Jr., Non-Linear Numerical Studies of the Tearing Mode. Ph.D. Thesis, Lawrence Livermore Laboratory, Livermore, Calif ., UCRL-52399 (1978). 110-145 (1980).

(107) D. Schnack and J. Killeen, Nonlinear, Two-Dimensional Magnetohydrodynamic Calculations, J. Comput. Phys. 35, 110-145 (1980).

(108) Weston M. Stacey, Jr., Fusion Plasma Analysis (John Wiley and Sons, New York, 1981), pp. 77-93, 235-262, 360 .

(109) Eli Turkel, Numerical Methods for Large-Scale, Time-Dependent Partial Differential Equations, in Computational Fluid Dymomics, edited by Wolfgang 
Kollman, (Hemisphere Publishing Corporation, Washington, 1980), Vol. 2, pp. 127-262.

(110) M. van Veldhuizen, D-Stability, SlAM J. Nomer. Anal. 18, 45-64 (1981).

(111) Hansjorg Wacker, E. Zarzer, and $\boldsymbol{W}$. Zulehner, Opt imal Stepsize Control for the Globalized Newton Method, in Cont inuation Methods, edited by Hansjorg Wacker (Academic Press, New York, 1978), pp. 249-276.

(112) H. H. Wang, A Parallel Method for Tridiagonal Equations, ACM Trans. Math. Soft. 7, 170-183 (1981).

(115) Layne T. Watson, Engineering Applications of the Chow-Yorke Algorithm. Appl. Math. and Comput. 9, 111-133 (1981).

(113) R. F. Warming and Richard M. Beam. An Extension of A-Stability to Alternating Direction Implicit Methods, BIT 19,395-417 (1979).

(114) R. F. Warming and Richard M. Beam, Recent Advances in the Development of Implicit Schemes for the Equations of Fluid Dynamics, in Lecture Notes in Physics, 141. Proc. Seventh International Conference on Nhmerical Methods in Fluid Dymamics, Stanford, 1980 (Springer-Verlag, Berlin, 1981), pp. 429-433.

(116) Alan M. Winslow, Adaptive Mesh Zoning by the Equipotential Method, Lawrence Livermore Laboratory, Livermore, Calif., UCID-19062 (1981). 
(117) Michael C. Wirth, On the Automation of Computational Physics, Ph.D. Thesis, Lawrence Livermore National Laboratory, Livermore, Calif., UCRL-52996 $(1980)$ 


\title{
DEVELOPMENT OF A MAGNETOHYDRODYNAMIC CODE
}

\section{FOR AXISYMMETRIC, HIGH $-\beta$ PLASMAS \\ WITH COMPLEX MAGNETIC FIELDS}

\author{
Grant 0. Cook, Jr. \\ Department of Physics and Astronomy \\ Ph.D. Degree, December, 1982
}

\begin{abstract}
The details of the development of a new two-dimensional MHD code are given. Included in the one-fluid, two-temperature model are the classical Braginski transport coefficients with viscous effects ignored, Saha-Boltzmann dissociation and partial ionization physics, and a radiation loss mechanism. Physical boundary conditions are used to close the system of equations. A complex set of current-carrying coils drive the plasma. Spline collocation spatially discretizes the overall system, and a "one-leg" method integrates the resulting ODEs in time. To solve the system, the driving coils are time split from the plasma model, and the matrix representation of the latier is split to yield two efficient "one-dimensional" problems. A generalized Newton method is proposed for solving the nonlinear discretized plasma equations. The code development problem is then addressed more generally by automating the algebraic work required to perform the discretizations and other transformations of the initial equations. With considerable capability being added by this work. MACSYMA was used to perform the algebraic task. Further code development tasks which are not yet automated are also discussed.
\end{abstract}

COMMITTEE APPROVAL:

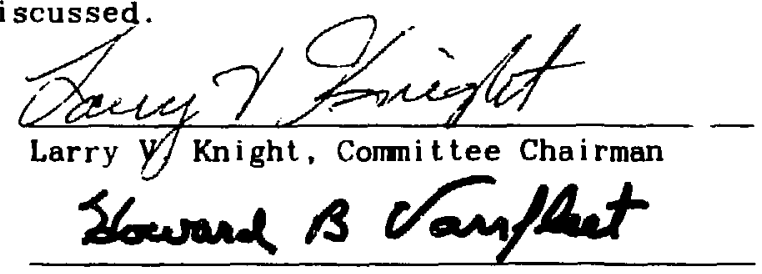

Howard B. Vanf leet, Committee Member
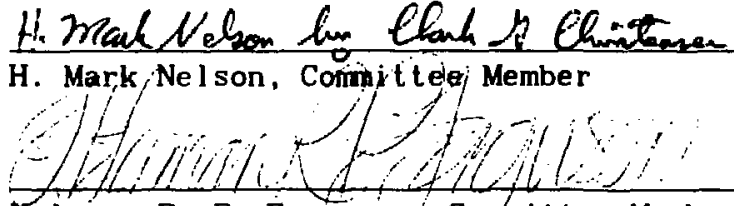

Hel aman R. P. Ferguson, Comit tee Member

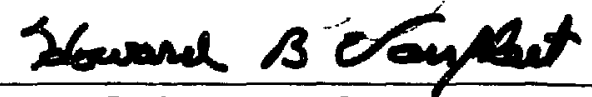

Howard B. Vanfleet, Department Chairman 
Technical Information Department - Lawrence Livermore National Laboratory University of California - Livermore, California 94550

\section{First Class Mail}

